\title{
Metal-free Domino Oligocyclization Reactions of Enynals and Enynones with Molecular Oxygen
}

Alireza Abbasi Kejani, ${ }^{\dagger}$ Hormoz Khosravi, ${ }^{\dagger}$ Frank Rominger, ${ }^{\star}$ Saeed Balalaie, ${ }^{*}, \dagger, \S$ Bernhard Breit*,ll

${ }^{\dagger}$ Peptide Chemistry Research Center, K. N. Toosi University of Technology, P.O. Box 158754416, Tehran, Iran

* Medical Biology Research Center, Kermanshah University of Medical Sciences, Kermanshah, Iran

${ }^{\S}$ Institut für Organische Chemie, Albert-Ludwigs-Universität Freiburg Albertstrasse 21, 79104 Freiburg im Breisgau, Germany

$\S$ Organisch-Chemisches Institut der Universität Heidelberg, Im Neuenheimer Feld 270, D69120 Heidelberg, Germany

\section{Table of content}

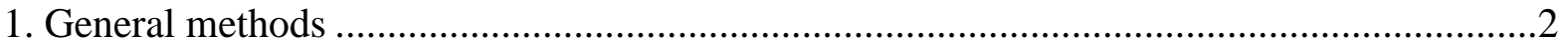

2. General Procedure for the Preparation of Substrate 1,6-enynes ........................................2

3. General Procedure for the Preparation of Substrate Enynals.............................................5

4. General Procedure for the Preparation of Substrate Enynones...........................................5

5. General Procedure of the Radical Domino Cyclization of Enynal 1 with Oxygen ..............6

6. General Procedure of the Radical Domino Cyclization of Enynone 3 with Oxygen ............6

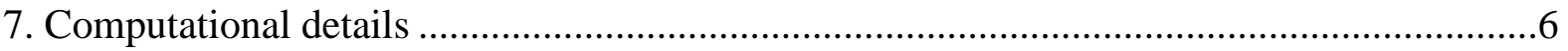

8. The spectroscopic data $\left({ }^{1} \mathrm{H},{ }^{13} \mathrm{C}\right.$ NMR, and HRMS $)$ of compounds....................................

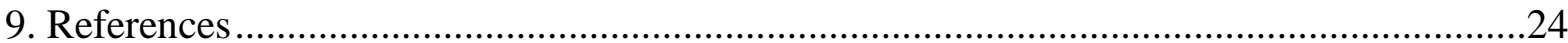

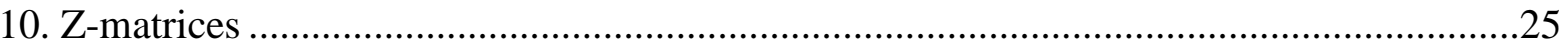

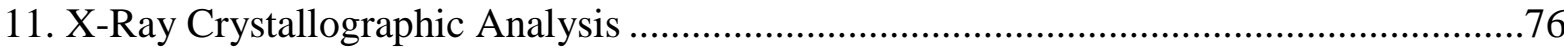

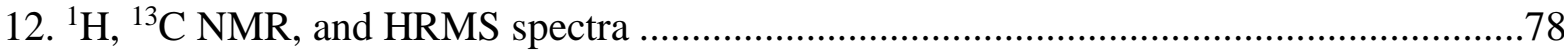




\section{General methods}

Commercially available reagents were purchased in a reagent grade and used without purification. Reaction progress was monitored by thin layer chromatography (TLC) using aluminum coated plates of silica gel 60-F254 and UV light of $254 \mathrm{~nm}$ for detection. Products were purified via flash column chromatography using silica gel 63-200 mesh. Melting point was presented by Electerothermal 9100 apparatus and are uncorrected. ${ }^{1} \mathrm{H}$ and ${ }^{13} \mathrm{C} \mathrm{NMR}$ spectroscopy were recorded on a Bruker 500, 400, $300 \mathrm{MHz}$ and 125, 100, $75 \mathrm{MHz}$ respectively. Chemical shifts are given parts per million $(\delta, \mathrm{ppm})$ and coupling constants are reported in Hertz $(J, H z)$. High resolution mass (ESI-HRMS) was obtained by Apex Qe FTICR spectrometer ( University of Heidelberg), and on a LCQ Advantage or Exactive mass spectrometer from Thermo Fisher Scientific Inc ( University of Freiburg). In some cases, Water LCT Premier ${ }^{\mathrm{TM}}$ XE mass spectrometer was used. X-ray crystal data were collected on Bruker APEX-II Quazar area detector.

\section{General Procedure for the Preparation of Substrate 1,6-enynes}

\section{General Procedure I $\left(\mathrm{X}=\mathrm{N}-\mathrm{Ts}, \mathrm{N}-\mathrm{SO}_{2} \mathrm{Me}, \mathrm{N}-\mathrm{SO}_{2} \mathrm{Naph}\right)^{1}$}

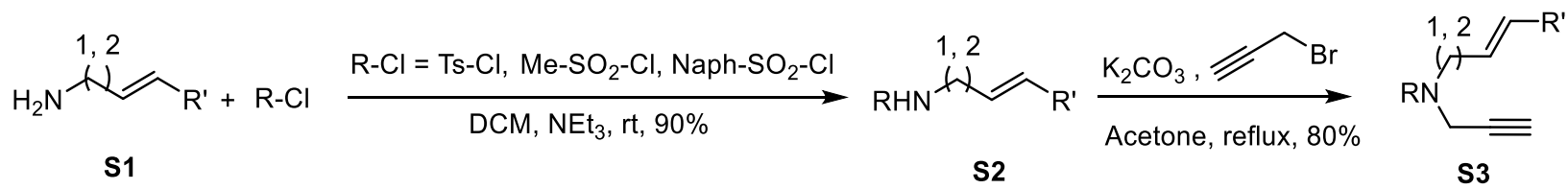
$\mathrm{R}^{\prime}=\mathrm{Me}, \mathrm{H}$

Step 1: Allylamine derivatives S1 (30.0 mmol) and $\mathrm{Et}_{3} \mathrm{~N}(12.54 \mathrm{~mL}, 90.0 \mathrm{mmol})$ were dissolved in $10 \mathrm{~mL}$ DCM and the mixture was cooled to $0{ }^{\circ} \mathrm{C} . p$-Toluenesulfonyl chloride or methanesulfonyl chloride, 2-nephthalenesulfonyl chloride $(30.0 \mathrm{mmol})$ was added portionwise to the solution, and the mixture was stirred at room temperature overnight. The mixture was treated with a saturated $\mathrm{NH}_{4} \mathrm{Cl}$ solution and then was extracted with ethyl acetate. The organic layer was washed with water and brine, dried over anhydrous $\mathrm{Na}_{2} \mathrm{SO}_{4}$ and concentrated to afford the protected allylamine $\mathbf{S 2}$ (90\% yield) which was used without further purification.

Step 2: The protected allylamine $\mathbf{S 2}$ resulting from the previous step (20 mmol, 1 equiv.), $\mathrm{K}_{2} \mathrm{CO}_{3}$ (3.87 g, $28.0 \mathrm{mmol}, 1.4$ equiv.) and acetone (0.4 M) were added to a oven dried flask. The corresponding propargyl bromide $(2.27 \mathrm{ml}, 30 \mathrm{mmol}, 1.5$ equiv.) was added and the reaction mixture was heated under reflux in the boil bath overnight. Then, the mixture was cooled to rt and the salts were filtered. Solvent was removed under vacuum, and the residue was extracted with DCM. The organic layer was washed with water and brine, dried over 
anhydrous $\mathrm{Na}_{2} \mathrm{SO}_{4}$ and concentrated to afford the 1,6-enyne $\mathbf{S 3}$ (80\% Yield) which was used without further purification.

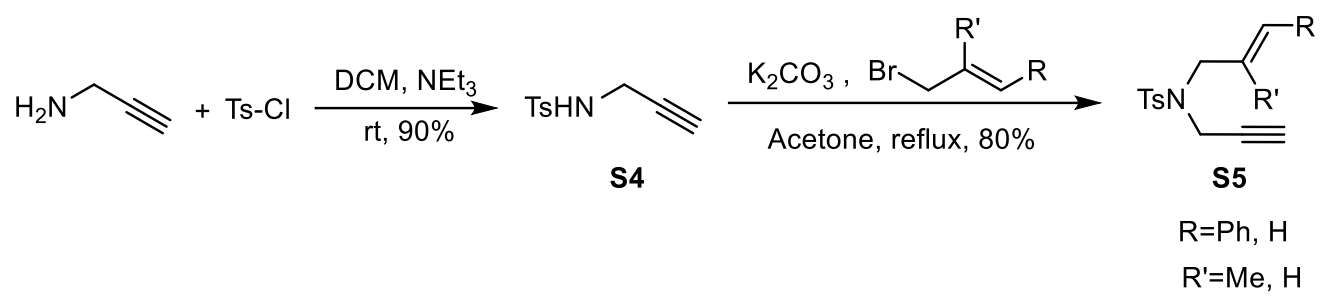

Step 1: Propargylmine $(1.65 \mathrm{~g}, 30.0 \mathrm{mmol})$ and $\mathrm{Et}_{3} \mathrm{~N}(12.7 \mathrm{~mL}, 90.0 \mathrm{mmol})$ were dissolved in $10 \mathrm{~mL}$ DCM and the mixture was cooled to $0{ }^{\circ} \mathrm{C}$. p-Toluenesulfonyl chloride $(5.70 \mathrm{~g}, 30.0$ mmol) was added portionwise to the solution, and the mixture was stirred at room temperature overnight. The mixture was treated with a saturated $\mathrm{NH}_{4} \mathrm{Cl}$ solution and then was extracted with ethyl acetate. The organic layer was washed with water and brine, dried over anhydrous $\mathrm{Na}_{2} \mathrm{SO}_{4}$ and concentrated to afford the $N$-tosyl- $N$-propargylamine $\mathbf{S 4}$ (90\% yield) which was used without further purification.

Step 2: The $N$-tosyl- $N$-propargylamine $\mathbf{S} 4$ resulting from the previous step ( $20 \mathrm{mmol}, 1$ equiv.), $\mathrm{K}_{2} \mathrm{CO}_{3}(3.87 \mathrm{~g}, 28.0 \mathrm{mmol}, 1.4$ equiv.) and acetone (0.4 M) were added to a oven dried flask. The corresponding drivatives allyl bromide (30 mmol, 1.5 equiv) was added and the reaction mixture was heated under reflux in the oil bath overnight. Then, the mixture was cooled to rt and the salts were filtered. Solvent was removed under vacuum, and the residue was extracted with DCM. The organic layer was washed with water and brine, dried over anhydrous $\mathrm{Na}_{2} \mathrm{SO}_{4}$ and concentrated to afford the 1,6-enyne $\mathbf{S 5}$ (80\% Yield) which was used without further purification.

\section{General Procedure II $(\mathrm{X}=\mathrm{N}-\mathrm{Cbz})^{2}$}

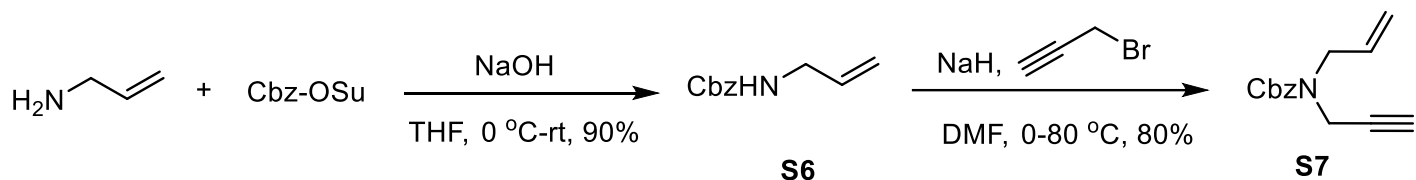

Step 1: To a solution of allylamine $(2.62 \mathrm{ml}, 35.0 \mathrm{mmol}, 1.0$ equiv. $)$ in $\mathrm{THF}(20 \mathrm{~mL})$ at $0{ }^{\circ} \mathrm{C}$ was added $\mathrm{NaOH}$ aqueous solution $(16.00 \mathrm{ml}, 35.0 \mathrm{mmol}, 2 \mathrm{~mol} / \mathrm{L})$ for $30 \mathrm{~min}$. Then a solution of Cbz-OSu (35.0 mmol, 1.0 equiv.) in $20 \mathrm{~mL}$ THF was added dropwise by constant pressure funnel under vigorous stirring. The mixture was stirred at room temperature overnight. Extraction with AcOEt and water $(3 \times 10 \mathrm{~mL})$. The organic layer was dried over $\mathrm{Na}_{2} \mathrm{SO}_{4}$ and concentrated to afford the Cbz-allylamine S6 (91\% yield) which was used without further purification. 
Step 2: The Cbz-allylamine $\mathbf{S 6}$ resulting from the previous step (3.82 g, $20.0 \mathrm{mmol}, 1.0$ equiv.) in DMF $(20 \mathrm{~mL})$ at $0{ }^{\circ} \mathrm{C}$ was added $\mathrm{NaH}(0.58 \mathrm{~g}, 24.0 \mathrm{mmol}, 1.2$ equiv. $)$ for $40 \mathrm{~min}$. Then a solution of propargylamine $(2.27 \mathrm{ml}, 30.0 \mathrm{mmol}, 1.5$ equiv.) in $10 \mathrm{~mL}$ DMF was added dropwise by constant pressure funnel under vigorous stirring. Then the mixture was stirred and heated to $80{ }^{\circ} \mathrm{C}$ in the oil bath for $5 \mathrm{~h}$. Extraction with AcOEt and water ( $\left.3 \times 10 \mathrm{~mL}\right)$. The organic layer was dried over $\mathrm{Na}_{2} \mathrm{SO}_{4}$ and concentrated to afford the 1,6-enyne $\mathbf{S 7}$ (80\% Yield) which was used without further purification.

\section{General Procedure III $(\mathrm{X}=\mathbf{O})^{3}$}

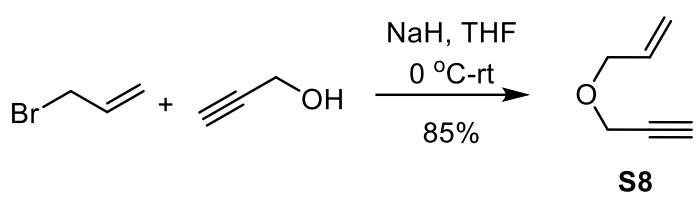

$\mathrm{NaH}$ (2 equiv, 60\% dispersion mineral oil, previously washed with $n$-hexane) and THF (0.4 $\mathrm{M}$ ) were added to a round bottom flask. The reaction was cooled to $0{ }^{\circ} \mathrm{C}$, and the corresponding propargyl alcohol (1.15 $\mathrm{ml}, 20 \mathrm{mmol})$ was added dropwise. The mixture was stirred for $1 \mathrm{~h}$ at rt. Then, the mixture was cooled to $0{ }^{\circ} \mathrm{C}$ and was kept at this temperature during the addition of the allyl bromide ( $2.59 \mathrm{ml}, 30 \mathrm{mmol}, 1.5$ equiv.). Next, it was stirred for 2 hours at rt. When the starting propargyl alcohol was consumed, water was added dropwise at $0{ }^{\circ} \mathrm{C}$. The mixture was extracted with AcOEt, the combined organic layers were washed with brine and dried over anhydrous $\mathrm{Na}_{2} \mathrm{SO}_{4}$ and concentrated to afford the 1,6-enyne $\mathbf{S 8}$ (85\% Yield) which was used without further purification.

\section{General Procedure IV $\left(\mathrm{X}=\mathrm{C}\left(\mathrm{CO}_{2} \mathrm{Me}\right)_{2}\right)$}

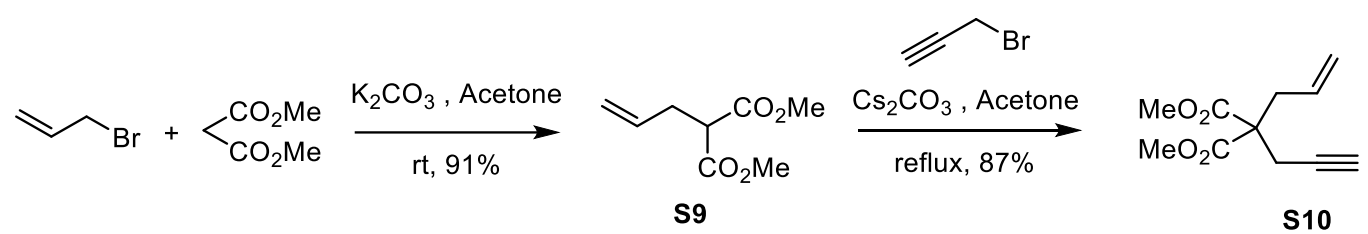

Step 1: Dimethyl malonate $(1 \mathrm{~mL}, 8.8 \mathrm{mmol}, 1.5$ equiv. $)$ and allyl bromide $(0.5 \mathrm{~mL}, 5.8$ mmol, 1 equiv.) were added to a solution of potassium carbonate ( $2.4 \mathrm{~g}, 17.4 \mathrm{mmol}, 3$ equiv $)$ in acetone $(30 \mathrm{~mL})$. The reaction mixture was allowed to stir for overnight at room temperature. The mixture was treated with a saturated $\mathrm{NH}_{4} \mathrm{Cl}$ solution and then was extracted dichloromethane. The organic layer was washed with water and brine, dried over anhydrous $\mathrm{Na}_{2} \mathrm{SO}_{4}$ and concentrated to afford the Dimethyl 2-allylmalonate $\mathbf{S 9}$ (91\% yield) which was used without further purification. ${ }^{4}$ 
Step 2: Dimethyl 2-allylmalonate S9 (2.54 g, 20.0 mmol, 1 equiv.), $\mathrm{Cs}_{2} \mathrm{CO}_{3}$ (9.77 g, 30 mmol, 1.5 equiv.) and acetone $(0.4 \mathrm{M})$ were added to a flask. The corresponding propargyl bromide ( $2.27 \mathrm{ml}, 30 \mathrm{mmol}, 1.5$ equiv.) was added and the reaction mixture was heated under reflux in the oil bath overnight. Then, the mixture was cooled to rt and the salts were filtered. Solvent was removed under vacuum, and the residue was extracted with DCM. The organic layer was washed with water and brine, dried over anhydrous $\mathrm{Na}_{2} \mathrm{SO}_{4}$ and concentrated to afford the 1,6-enyne S10 (87\% yield) which was used without further purification. ${ }^{3}$

\section{General Procedure for the Preparation of Substrate Enynals}

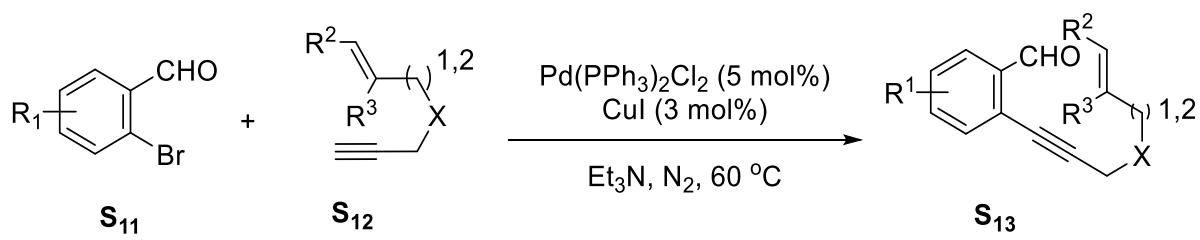

To a solution of the 2-bromobenzaldehyde S11 (3 mmol, 1 equiv.), $\mathrm{PdCl}_{2}\left(\mathrm{PPh}_{3}\right)_{2}(5 \mathrm{~mol} \%)$, and $\mathrm{CuI}(3 \mathrm{~mol} \%)$ in $\mathrm{Et}_{3} \mathrm{~N}$ (15 mL) wase added enynes $\mathbf{S 1 2}$ (1.5 equiv.) was added at room temperature under $\mathrm{N}_{2}$ atmosphere. The reaction mixture was heated at $60{ }^{\circ} \mathrm{C}$ in the oil bath for 6-18 h, and monitored by TLC. After the reaction was completed, the mixture was cooled to room temperature, and was concentrated under vacuum. The residue was purified by column chromatography on silica gel (EtOAc / $n$-hexane) to afford the product S13.

\section{General Procedure for the Preparation of Substrate Enynones}<smiles>O=C(c1ccccc1)c1ccccc1I</smiles>

$\mathrm{S}_{14}$

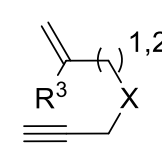

$\mathbf{S}_{15}$

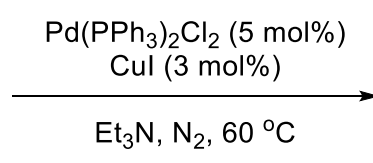

$\mathrm{Et}_{3} \mathrm{~N}, \mathrm{~N}_{2}, 60^{\circ} \mathrm{C}$

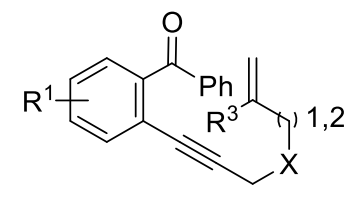

$S_{16}$

To a solution of the 2-iodobenzophenone $\mathbf{S 1 4}$ (3 mmol, 1 equiv.), $\mathrm{PdCl}_{2}\left(\mathrm{PPh}_{3}\right)_{2}$ (5 mol\%), and $\mathrm{CuI}(3 \mathrm{~mol} \%)$ in $\mathrm{Et}_{3} \mathrm{~N}$ (15 mL) wase added enynes $\mathbf{S 1 5}$ (1.5 equiv.) was added at room temperature under $\mathrm{N}_{2}$ atmosphere. The reaction mixture was heated at $60{ }^{\circ} \mathrm{C}$ in the oil bath for 6-18 h, and monitored by TLC. After the reaction was completed, the mixture was cooled to room temperature, and was concentrated under vacuum. The residue was purified by column chromatography on silica gel (EtOAc / $n$-hexane) to afford the product S16. 


\section{General Procedure of the Radical Domino Cyclization of Enynal 1 with Oxygen}

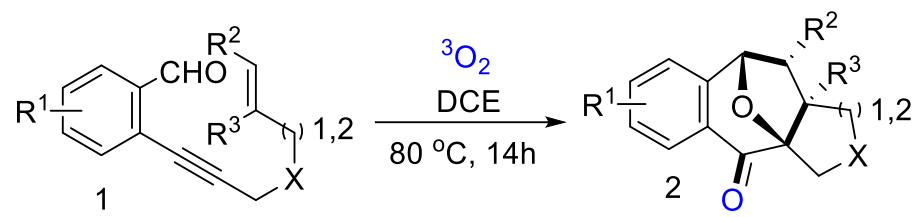

Enynal $1(0.2 \mathrm{mmol})$ was added to a schlenk flask in DCE $(1 \mathrm{~mL})$ and under oxygen atmosphere. The mixture was stirred at $80{ }^{\circ} \mathrm{C}$ in the oil bath for 14 hours. After completion of reaction as indicated by TLC, the solvent was evaporated under reduced pressure. The residue was purified by flash column chromatograph on silica gel (EtOAc / $n$-hexane) to afford the product 2.

\section{General Procedure of the Radical Domino Cyclization of Enynone 3 with Oxygen}
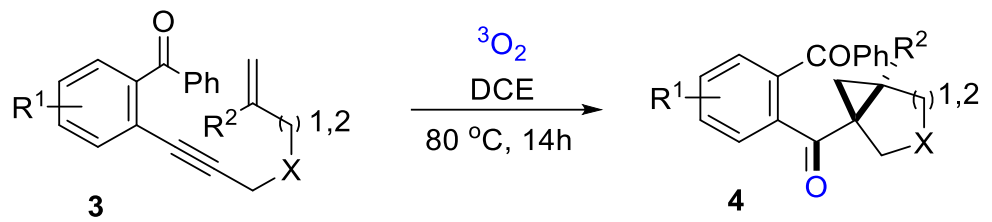

Enynone 3 (0.2 mmol) was added to a schlenk flask in DCE $(1 \mathrm{~mL})$ and under oxygen atmosphere. The mixture was stirred at $80{ }^{\circ} \mathrm{C}$ in the oil bath for 14 hours. After completion of reaction as indicated by TLC, the solvent was evaporated under reduced pressure. The residue was purified by flash column chromatograph on silica gel (EtOAc /n-hexane) to afford the product 4 .

\section{Computational details}

All the DFT calculations have been carried out with the Gaussian 09 package of program ${ }^{5}$ and visualization of computed structures was generated using CYLView. ${ }^{6}$ Full geometry optimization was performed with the B3LYP/6-31G(d,p) level in the gas phase (T=298 K, P=1 bar). ${ }^{7}$ The correctness of the optimized structures and transition states (TS) has been verified by harmonic vibrational frequency calculations. The intrinsic reaction coordinate (IRC) were obtained to determine TS structures are correct. The natural bond orbital (NBO) analysis was done using the B3LYP/6-311++G(d,p) on optimized structures in the gas phase. Single-point calculations and solvation energy corrections were performed on the gas-phase optimized structures using the 6-311++G(d,p) as the basis set and the IEFPCM model (with the DCE as the solvent) to refine the electronic energy $\left(\boldsymbol{E}_{S P}\right)$. The thermal vibrational corrections to the 
enthalpy $\left(\boldsymbol{H}_{\boldsymbol{D} Z}^{\boldsymbol{C o r r}}\right)$ and the electronic energy $\left(\boldsymbol{E}_{\boldsymbol{S P}}\right)$ were used to calculate the enthalpy of each structure based on the following equation: ${ }^{8}$

$H_{T Z}=E_{S P}+H^{C o r r}$

Electron transfer from $1 \mathrm{~b}$ and $3 \mathrm{~b}$ to related INT-V and INT-VII was investigated using MarcusHush theory, were the energy barriers of electron transfer can be estimated using the following equations: ${ }^{9}$

$$
\begin{gathered}
\Delta G^{\ddagger} \approx\left(\frac{\lambda_{\mathrm{i}}}{4}\right) \times\left(1+\frac{\Delta G}{\lambda_{\mathrm{i}}}\right)^{2} \\
\lambda_{\mathrm{i}}=\left(\frac{1}{2}\right) \times\left(\lambda_{\mathrm{i}(\text { donor })}+\lambda_{\mathrm{i} \text { (acceptor })}\right) \\
\lambda_{\mathrm{i} \text { (species) }}=\left(E_{N}\left(R_{N-1}\right)-E_{N}\left(R_{N}\right)+\left(E_{N-1}\left(R_{N}\right)-E_{N-1}\left(R_{N-1}\right)\right)\right.
\end{gathered}
$$

- $\quad \lambda_{\mathrm{i}}$ is the internal reorganization energy

- $\Delta G$ is the free energy change of the reaction and $\Delta G^{\ddagger}$ is the activation free energy

- $E_{N}\left(R_{N}\right)$ and $E_{N-1}\left(R_{N-1}\right)$ are the energies donor and acceptor, respectively in their respective geometries; $E_{N}\left(R_{N-1}\right)$ is the single point energy of the acceptor structure with one more electron, and $\left(E_{N-1}\left(R_{N}\right)\right.$ is the single point energy of the donor with one less electron.

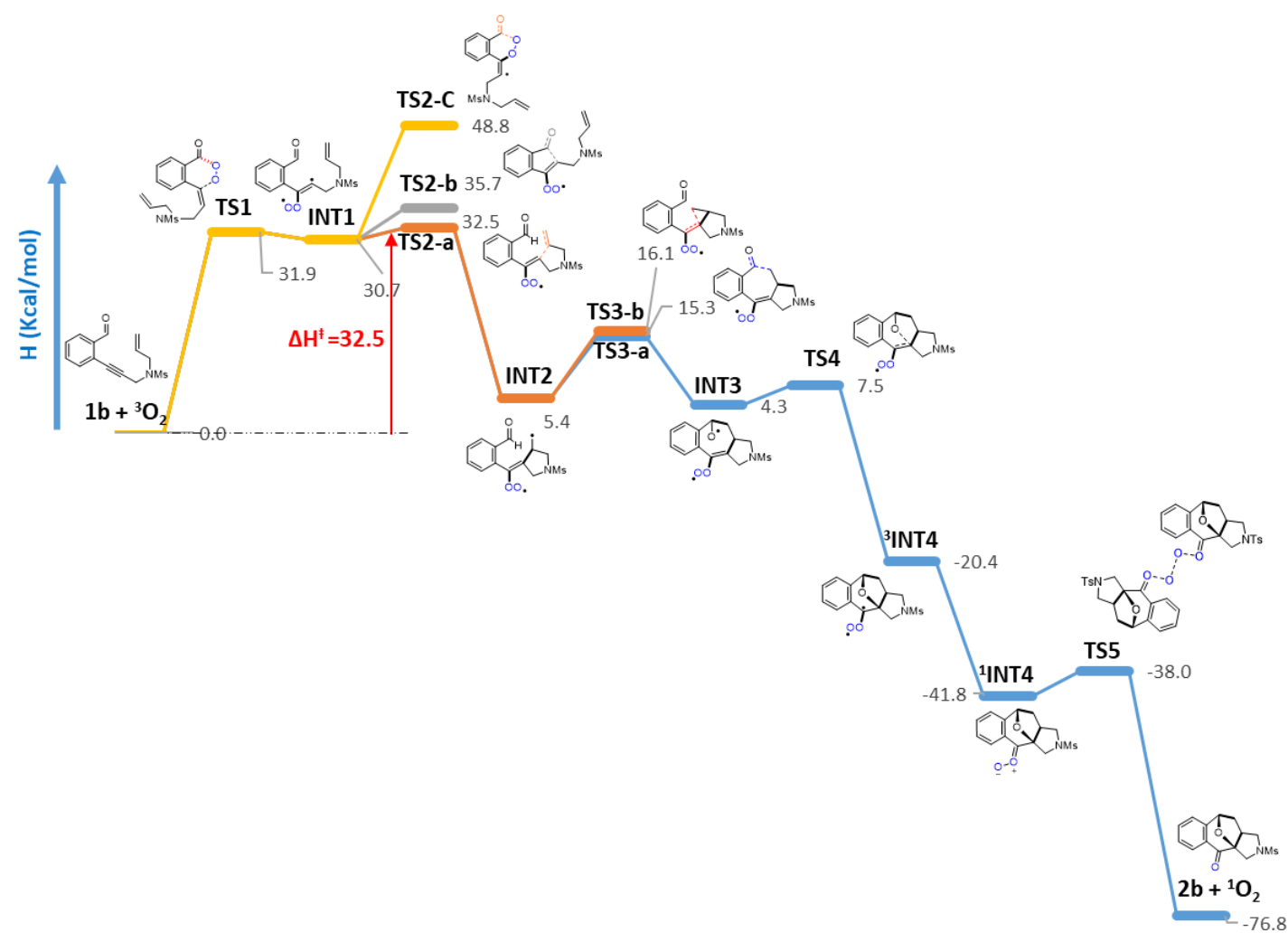

Figure S1. Potential energy surface for the direct addition of triplet molecular oxygen to $1 \mathrm{~b}$ as the substrate model. Structures have been optimized with B3LYP/6-31G(d,p) level of theory in the gas phase 


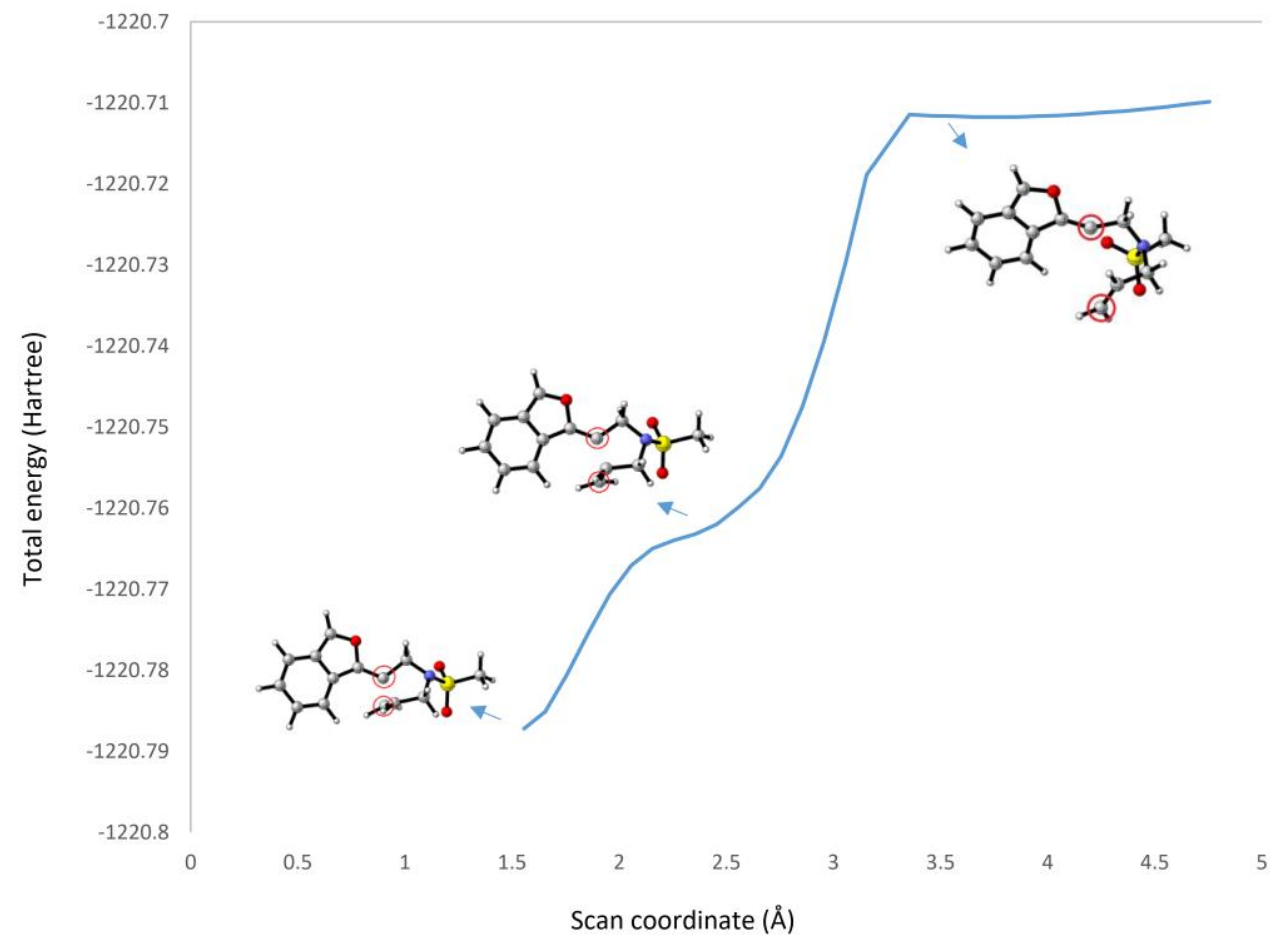

Figure S2. The potential energy surface (PES) scan related to [2+1] intramolecular cycloaddition reaction has been carried out with B3LYP/6-31G(d,p) level of theory in gas phase 


\section{The spectroscopic data $\left({ }^{1} \mathrm{H},{ }^{13} \mathrm{C}\right.$ NMR, and HRMS $)$ of compounds}

1a: $N$-Allyl- $N$-(3-(2-formylphenyl)prop-2-yn-1-yl)-4-methylbenzenesulfonamide

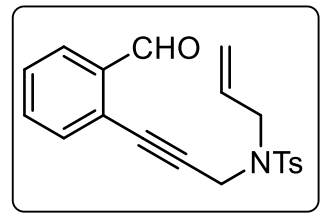

Yellow oil, (689 mg, yield 65\%), (silica gel, $n$-hexane/EtOAc $=7: 1) ;{ }^{1} \mathbf{H}$

NMR $\left(400 \mathrm{MHz}, \mathrm{CDCl}_{3}\right): \delta_{\mathrm{H}}(\mathrm{ppm})=9.91(\mathrm{~s}, 1 \mathrm{H}), 7.84(\mathrm{~d}, J=7.7 \mathrm{~Hz}$,

1H), $7.76(\mathrm{~d}, J=8.1 \mathrm{~Hz}, 2 \mathrm{H}), 7.51(\mathrm{t}, J=7.2 \mathrm{~Hz}, 1 \mathrm{H}), 7.42(\mathrm{t}, J=7.5 \mathrm{~Hz}$,

$1 \mathrm{H}), 7.24(\mathrm{~d}, J=7.3 \mathrm{~Hz}, 1 \mathrm{H}), 7.22(\mathrm{~d}, J=8.1 \mathrm{~Hz}, 2 \mathrm{H}), 5.87-5.76(\mathrm{~m}, 1 \mathrm{H}), 5.34(\mathrm{~d}, J=17.5$ $\mathrm{Hz}, 1 \mathrm{H}), 5.30$ (d, $J=10.3 \mathrm{~Hz}, 1 \mathrm{H}), 4.39$ (s, 2H), $3.92(\mathrm{~d}, J=6.4 \mathrm{~Hz}, 2 \mathrm{H}), 2.26(\mathrm{~s}, 3 \mathrm{H}) ;{ }^{13} \mathbf{C}$ NMR $\left(100 \mathrm{MHz}, \mathrm{CDCl}_{3}\right): \delta_{\mathrm{C}}(\mathrm{ppm})=190.7,143.9,135.8,135.7,133.6,133.4,131.9,129.7$, 128.9, 127.7, 127.1, 125.6, 120.2, 89.1, 81.4, 49.6, 36.7, 21.4; HRMS-ESI (m/z): calcd. for $\mathrm{C}_{40} \mathrm{H}_{38} \mathrm{~N}_{2} \mathrm{O}_{6} \mathrm{~S}_{2} \mathrm{Na}[2 \mathrm{M}+\mathrm{Na}]^{+} 729.2063$, found 729.2061 .

1b: $N$-Allyl- $N$-(3-(2-formylphenyl)prop-2-yn-1-yl)methanesulfonamide

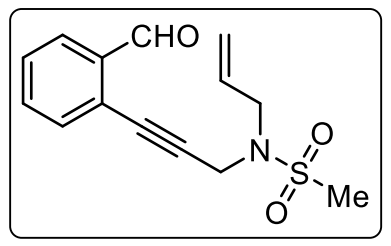

Yellow oil, (532 mg, yield 64\%), (silica gel, $n$-hexane/EtOAc = 8:1); ${ }^{1} \mathbf{H}$ NMR $\left(300 \mathrm{MHz}, \mathrm{CDCl}_{3}\right): \delta_{\mathrm{H}}(\mathrm{ppm})=10.41(\mathrm{~s}, 1 \mathrm{H}), 7.91(\mathrm{dd}, J=$ 7.6, $1.6 \mathrm{~Hz}, 1 \mathrm{H}), 7.62-7.49(\mathrm{~m}, 3 \mathrm{H}), 5.94-5.80(\mathrm{~m}, 1 \mathrm{H}), 5.42(\mathrm{dd}$, $J=17.1,1.4 \mathrm{~Hz}, 1 \mathrm{H}), 5.35(\mathrm{dd}, J=10.0,1.3 \mathrm{~Hz}, 1 \mathrm{H}), 4.38(\mathrm{~s}, 2 \mathrm{H})$, $4.01(\mathrm{~d}, J=6.3 \mathrm{~Hz}, 2 \mathrm{H}), 3.01(\mathrm{~s}, 3 \mathrm{H}) ;{ }^{13} \mathbf{C ~ N M R}\left(75 \mathrm{MHz}, \mathrm{CDCl}_{3}\right): \delta_{\mathrm{C}}(\mathrm{ppm})=190.8,136.2$, 133.7, 133.8, 131.9, 129.3, 128.8, 124.6, 120.2, 89.5, 82.1, 49.5, 38.7, 36.6; Mass-ESI (m/z): calcd. for $\mathrm{C}_{14} \mathrm{H}_{15} \mathrm{NO}_{3} \mathrm{SNa}[\mathrm{M}+\mathrm{Na}]^{+}$300.0670, found 300.0661; Anal calcd: C, 60.63; H, 5.45; N, 5.05; S, 11.56. found: C, 60.66; H, 5.47; N, 5.06; S, 11.58 .

\section{1c: Benzyl allyl(3-(2-formylphenyl)prop-2-yn-1-yl)carbamate}

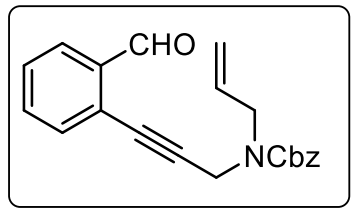

Yellow oil, (610 mg, yield 61\%), (silica gel, $n$-hexane/EtOAc $=7: 1)$; ${ }^{1} \mathbf{H}$ NMR $\left(300 \mathrm{MHz}, \mathrm{CDCl}_{3}\right): \delta_{\mathrm{H}}(\mathrm{ppm})=10.47(\mathrm{~s}, 1 \mathrm{H}), 7.92(\mathrm{dt}, J=$ 7.7, $1.1 \mathrm{~Hz}, 1 \mathrm{H}), 7.55(\mathrm{~d}, J=7.2 \mathrm{~Hz}, 1 \mathrm{H}), 7.46(\mathrm{~d}, J=7.8 \mathrm{~Hz}, 1 \mathrm{H}), 7.42$ $-7.35(\mathrm{~m}, 5 \mathrm{H}), 7.35-7.32(\mathrm{~m}, 2 \mathrm{H}) 5.90-5.80(\mathrm{~m}, 1 \mathrm{H}), 5.21$ (brs, $1 \mathrm{H}), 5.12-5.24(\mathrm{~m}, 2 \mathrm{H})$, $4.10(\mathrm{~d}, J=5.7 \mathrm{~Hz}, 2 \mathrm{H}), 3.87-3.77(\mathrm{~m}, 2 \mathrm{H}) ;{ }^{13} \mathbf{C} \mathbf{N M R}\left(75 \mathrm{MHz}, \mathrm{CDCl}_{3}\right): \delta_{\mathrm{C}}(\mathrm{ppm})=191.5$, 156.3, 136.5, 136.2, 134.5, 133.7, 133.5, 132.9, 128.8, 128.5, 128.1, 127.9, 127.3, 116.1, 91.9, 77.3, 67.7, 66.8, 43.5; HRMS-ESI $(\mathrm{m} / \mathrm{z})$ : calcd. for $\mathrm{C}_{21} \mathrm{H}_{20} \mathrm{NO}_{3}[\mathrm{M}+\mathrm{H}]^{+}$334.1438, found 334.1433 . 


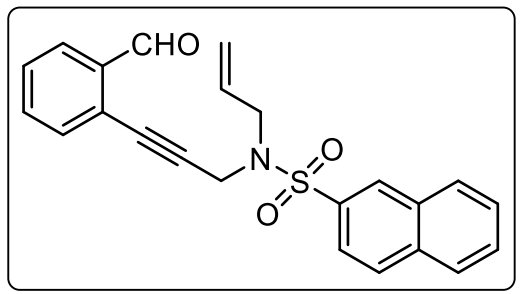

Yellow oil, (771 mg, yield 66\%), (silica gel, $n$-hexane/EtOAc $=7: 1) ;{ }^{1} \mathbf{H}$ NMR $\left(500 \mathrm{MHz}, \mathrm{CDCl}_{3}\right): \delta_{\mathrm{H}}(\mathrm{ppm})=9.91(\mathrm{~s}, 1 \mathrm{H})$, $8.47(\mathrm{~d}, J=1.8 \mathrm{~Hz}, 1 \mathrm{H}), 7.88(\mathrm{~d}, J=1.7 \mathrm{~Hz}, 1 \mathrm{H}), 7.86-7.85$ $(\mathrm{m}, 2 \mathrm{H}), 7.77(\mathrm{~d}, J=8.7 \mathrm{~Hz}, 1 \mathrm{H}), 7.67(\mathrm{dd}, J=7.6,1.7 \mathrm{~Hz}$, $1 \mathrm{H}), 7.56(\mathrm{ddd}, J=8.2,6.9,1.3 \mathrm{~Hz}, 1 \mathrm{H}), 7.50(\mathrm{ddd}, J=8.2,6.9,1.3 \mathrm{~Hz}, 1 \mathrm{H}), 7.32-7.28$ (m, $1 \mathrm{H}), 7.26(\mathrm{dd}, J=7.5,1.5 \mathrm{~Hz}, 2 \mathrm{H}), 6.78(\mathrm{dd}, J=7.5,1.6 \mathrm{~Hz}, 1 \mathrm{H}) .5 .88-5.77(\mathrm{~m}, 1 \mathrm{H}), 5.34$ $(\mathrm{dd}, J=16.9,1.5 \mathrm{~Hz}, 1 \mathrm{H}), 5.29$ (dd, $J=10.0,1.5 \mathrm{~Hz}, 1 \mathrm{H}), 4.44$ (s, 2H), 3.99 (d, $J=6.5 \mathrm{~Hz}$, $2 \mathrm{H}) ;{ }^{13} \mathbf{C ~ N M R}\left(125 \mathrm{MHz}, \mathrm{CDCl}_{3}\right): \delta_{\mathrm{C}}(\mathrm{ppm})=190.5,135.8,135.7,135.0,133.4,133.2,132.2$, 132.0, 129.3, 129.2, 129.2, 128.9, 128.7, 127.9, 127.5, 127.3, 125.2, 122.9, 120.3, 88.9, 81.6, 49.8, 36.9; HRMS-ESI (m/z): calcd. for $\mathrm{C}_{46} \mathrm{H}_{38} \mathrm{~N}_{2} \mathrm{O}_{6} \mathrm{~S}_{2} \mathrm{Na}[2 \mathrm{M}+\mathrm{Na}]^{+}$801.2063, found 801.2060.

\section{1e: Dimethyl 2-allyl-2-(3-(2-formylphenyl)prop-2-yn-1-yl)malonate}

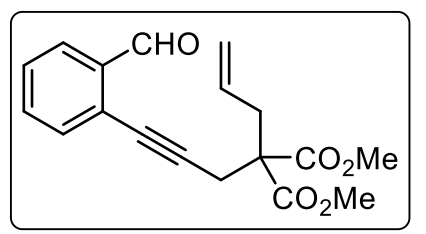

Yellow oil, (566 mg, yield 60\%), (silica gel, $n$-hexane/EtOAc $=$ 6:1); ${ }^{1} \mathbf{H}$ NMR $\left(400 \mathrm{MHz}, \mathrm{CDCl}_{3}\right): \delta_{\mathrm{H}}(\mathrm{ppm})=10.45(\mathrm{~s}, 1 \mathrm{H}), 7.89$ $(\mathrm{dd}, J=7.83,1.39 \mathrm{~Hz}, 1 \mathrm{H}), 7.53-7.50(\mathrm{~m}, 2 \mathrm{H}), 7.43-7.39(\mathrm{~m}$, 1H), $5.72-5.62(\mathrm{~m}, 1 \mathrm{H}), 5.20(\mathrm{dd}, J=17.0,1.9 \mathrm{~Hz}, 1 \mathrm{H}), 5.17(\mathrm{dd}, J=10.1,2.0 \mathrm{~Hz}, 1 \mathrm{H}), 3.77$ (s, 6H), 3.10 (s, 2H).; ${ }^{13} \mathbf{C}$ NMR (100 MHz, $\left.\mathrm{CDCl}_{3}\right): \delta_{\mathrm{C}}(\mathrm{ppm})=191.7,170.2,136.3,134.0$, 133.7, 133.6, 131.7, 128.5, 127.2, 126.8, 120.2, 117.7, 92.0, 79.4, 57.2, 52.9, 37.1, 24.1; HRMS-ESI (m/z): calcd. for $\mathrm{C}_{36} \mathrm{H}_{36} \mathrm{O}_{10} \mathrm{Na}[2 \mathrm{M}+\mathrm{Na}]^{+}$651.2201, found 651.2202.

\section{1f: 2-(3-(Allyloxy)prop-1-yn-1-yl)benzaldehyde}

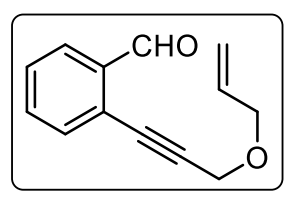

Yellow oil, (372 mg, yield 62\%), (silica gel, $n$-hexane/EtOAc $=8: 1) ;{ }^{1} \mathbf{H}$ NMR $\left(300 \mathrm{MHz}, \mathrm{CDCl}_{3}\right): \delta_{\mathrm{H}}(\mathrm{ppm})=10.54(\mathrm{~s}, 1 \mathrm{H}), 7.92(\mathrm{~d}, J=7.9 \mathrm{~Hz}$, $1 \mathrm{H}), 7.59-7.55(\mathrm{~m}, 2 \mathrm{H}), 7.50-7.43(\mathrm{~m}, 1 \mathrm{H}), 6.04-5.88(\mathrm{~m}, 1 \mathrm{H}), 5.37$ $(\mathrm{dd}, J=17.2,1.6 \mathrm{~Hz}, 1 \mathrm{H}), 5.27$ (dd, $J=10.4,1.5 \mathrm{~Hz}, 1 \mathrm{H}), 4.46(\mathrm{~s}, 2 \mathrm{H}), 4.19-4.12(\mathrm{~m}, 2 \mathrm{H})$; ${ }^{13} \mathrm{C}$ NMR $\left(75 \mathrm{MHz}, \mathrm{CDCl}_{3}\right): \delta_{\mathrm{C}}(\mathrm{ppm})=191.5,140.4,136.1,133.8,133.7,133.5,128.9,127.3$, 118.2, 92.4, 77.2, 71.0, 57.8; HRMS-ESI (m/z): calcd. for $\mathrm{C}_{13} \mathrm{H}_{12} \mathrm{O}_{2}[\mathrm{M}+\mathrm{H}]^{+}$201.0910, found 201.0909 . 
1g: $N$-(But-3-en-1-yl)-N-(3-(2-formylphenyl)prop-2-yn-1-yl)-4-methylbenzene sulfonamide

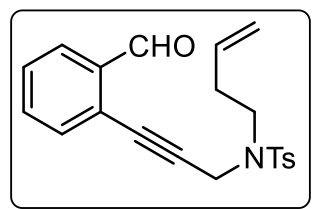

Yellow oil, (705 mg, yield 64\%), (silica gel, $n$-hexane/EtOAc $=7: 1) ;{ }^{1} \mathbf{H}$

NMR $\left(300 \mathrm{MHz}, \mathrm{CDCl}_{3}\right): \delta_{\mathrm{H}}(\mathrm{ppm})=9.88(\mathrm{~s}, 1 \mathrm{H}), 7.85(\mathrm{dd}, J=7.6,1.7$

$\mathrm{Hz}, 1 \mathrm{H}), 7.76(\mathrm{~d}, J=8.3 \mathrm{~Hz}, 2 \mathrm{H}), 7.51(\mathrm{td}, J=7.5,1.6 \mathrm{~Hz}, 1 \mathrm{H}), 7.44(\mathrm{~d}$, $J=7.5 \mathrm{~Hz}, 1 \mathrm{H}), 7.28-7.19(\mathrm{~m}, 4 \mathrm{H}), 5.90-5.73(\mathrm{~m}, 1 \mathrm{H}), 5.21-5.07(\mathrm{~m}, 2 \mathrm{H}), 4.45(\mathrm{~s}, 2 \mathrm{H})$, $3.36(\mathrm{t}, J=7.3 \mathrm{~Hz}, 2 \mathrm{H}), 2.46-2.39(\mathrm{~m}, 2 \mathrm{H}), 2.28(\mathrm{~s}, 3 \mathrm{H}) ;{ }^{13} \mathbf{C} \mathbf{N M R}\left(75 \mathrm{MHz}, \mathrm{CDCl}_{3}\right): \delta_{\mathrm{C}}$ $(\mathrm{ppm})=190.7,143.8,135.8,134.4,133.6,133.4,129.6,128.9,127.6,127.2,125.6,117.5$, 89.1, 81.4, 46.1, 37.2, 32.4, 21.4; HRMS-ESI (m/z): calcd. for $\mathrm{C}_{21} \mathrm{H}_{22} \mathrm{NO}_{3} \mathrm{~S}[\mathrm{M}+\mathrm{H}]^{+} 368.1315$, found 368.1314 .

1h: $N$-(But-3-en-1-yl)- $N$-(3-(2-formyl-4-methoxyphenyl)prop-2-yn-1-yl)-4 methylbenzene sulfonamide

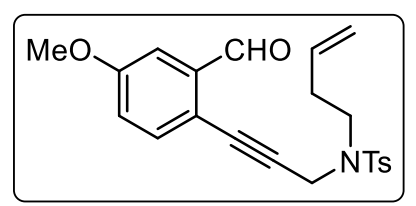

Yellow oil, (787 mg, yield 66\%), (silica gel, $n$-hexane/EtOAc $=7: 1$ ); ${ }^{1}$ H NMR (300 MHz, $\left.\mathrm{CDCl}_{3}\right): \delta_{\mathrm{H}}(\mathrm{ppm})=9.81(\mathrm{~s}, 1 \mathrm{H}), 7.74(\mathrm{~d}, J=$ $8.3 \mathrm{~Hz}, 2 \mathrm{H}), 7.30(\mathrm{~d}, J=2.8 \mathrm{~Hz}, 1 \mathrm{H}), 7.21(\mathrm{~d}, J=8.5 \mathrm{~Hz}, 2 \mathrm{H}), 7.15$ $(\mathrm{d}, J=8.6 \mathrm{~Hz}, 1 \mathrm{H}), 7.03(\mathrm{dd}, J=8.6,2.3 \mathrm{~Hz}, 1 \mathrm{H}), 5.92-5.68(\mathrm{~m}, 1 \mathrm{H}), 5.14(\mathrm{dd}, J=18.6,1.4$ $\mathrm{Hz}, 1 \mathrm{H}), 5.08$ (dd, $J=10.4,1.3 \mathrm{~Hz}, 1 \mathrm{H}), 4.41(\mathrm{~s}, 2 \mathrm{H}), 3.84(\mathrm{~s}, 3 \mathrm{H}), 3.33(\mathrm{t}, J=7.3 \mathrm{~Hz}, 2 \mathrm{H})$, $2.43-2.37(\mathrm{~m}, 2 \mathrm{H}), 2.29(\mathrm{~s}, 3 \mathrm{H}) ;{ }^{13} \mathbf{C} \mathbf{N M R}\left(75 \mathrm{MHz}, \mathrm{CDCl}_{3}\right): \delta_{\mathrm{C}}(\mathrm{ppm})=190.7,159.9,143.8$, 137.2, 135.7, 134.7, 129.6, 127.6, 127.1, 121.5, 117.5, 109.6, 87.4, 81.2, 55.7, 46.0, 37.2, 32.3, 21.4; HRMS-ESI (m/z): calcd. for $\mathrm{C}_{22} \mathrm{H}_{23} \mathrm{NO}_{4} \mathrm{SNa}[\mathrm{M}+\mathrm{Na}]^{+} 420.1251$, found 420.1243 .

1i: $N$-Allyl- $N$-(3-(2-formyl-4-methoxyphenyl)prop-2-yn-1-yl)-4-methylbenzenesulfonamide

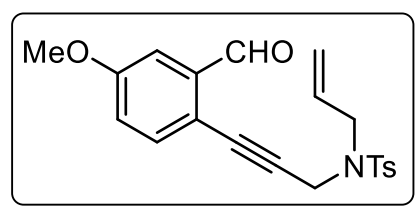

Yellow oil, (748 mg, yield 65\%), (silica gel, $n$-hexane $/$ EtOAc $=$ 7:1); ${ }^{1} \mathbf{H}$ NMR $\left(300 \mathrm{MHz}, \mathrm{CDCl}_{3}\right): \delta_{\mathrm{H}}(\mathrm{ppm})=9.87(\mathrm{~s}, 1 \mathrm{H}), 7.75$ $(\mathrm{d}, J=8.3 \mathrm{~Hz}, 2 \mathrm{H}), 7.31(\mathrm{~d}, J=2.7 \mathrm{~Hz}, 1 \mathrm{H}), 7.22(\mathrm{~d}, J=8.4 \mathrm{~Hz}$, 2H), $7.15(\mathrm{~d}, J=8.6 \mathrm{~Hz}, 1 \mathrm{H}), 7.04(\mathrm{dd}, J=8.6,2.8 \mathrm{~Hz}, 1 \mathrm{H}), 5.87-5.72(\mathrm{~m}, 1 \mathrm{H}), 5.33$ (dd, $J$ $=15.6,1.3 \mathrm{~Hz}, 1 \mathrm{H}), 5.28(\mathrm{dd}, J=8.4,1.3 \mathrm{~Hz}, 1 \mathrm{H}), 4.37(\mathrm{~s}, 2 \mathrm{H}), 3.90(\mathrm{~d}, J=6.41 \mathrm{~Hz}, 2 \mathrm{H}), 3.85$ $(\mathrm{s}, 3 \mathrm{H}), 2.30(\mathrm{~s}, 3 \mathrm{H}) ;{ }^{13} \mathbf{C} \mathbf{N M R}\left(75 \mathrm{MHz}, \mathrm{CDCl}_{3}\right): \delta_{\mathrm{C}}(\mathrm{ppm})=190.7,159.9,143.8,137.3$, 135.9, 134.7, 132.0, 129.6, 127.7, 127.1, 121.4, 120.1, 109.7, 87.4, 81.2, 55.7, 49.5, 36.7, 21.4; Mass-ESI (m/z): calcd. for $\mathrm{C}_{21} \mathrm{H}_{22} \mathrm{NO}_{4} \mathrm{~S}[\mathrm{M}+\mathrm{H}]^{+}$384.1270, found 384.1263; Anal calcd: $\mathrm{C}$, 65.78; H, 5.52; N, 3.65; S, 8.36. found: C, C, 65.80; H, 5.56; N, 3.69; S, 8.39. 
1j: $N$-Allyl- $N$-(3-(4-(benzyloxy)-2-formylphenyl)prop-2-yn-1-yl)-4-methylbenzene sulfonamide

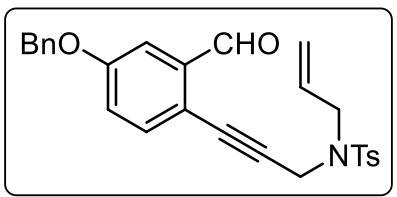

Yellow oil, (882 mg, yield 64\%), (silica gel, $n$-hexane/EtOAc $=8: 1$ ); ${ }^{1} \mathbf{H}$ NMR $\left(300 \mathrm{MHz}, \mathrm{CDCl}_{3}\right): \delta_{\mathrm{H}}(\mathrm{ppm})=9.85(\mathrm{~s}, 1 \mathrm{H}), 7.76(\mathrm{~d}, J=$ $8.3 \mathrm{~Hz}, 2 \mathrm{H}), 7.44-7.37(\mathrm{~m}, 6 \mathrm{H}), 7.22(\mathrm{~d}, J=8.0 \mathrm{~Hz}, 2 \mathrm{H}), 7.16(\mathrm{~d}, J$ $=8.5 \mathrm{~Hz}, 1 \mathrm{H}), 7.12(\mathrm{dd}, J=8.6,2.5 \mathrm{~Hz}, 1 \mathrm{H}), 5.89-5.74(\mathrm{~m}, 1 \mathrm{H}), 5.36-5.27(\mathrm{~m}, 2 \mathrm{H}), 5.11$ $(\mathrm{s}, 2 \mathrm{H}), 4.37(\mathrm{~s}, 2 \mathrm{H}), 3.91(\mathrm{~d}, J=6.4 \mathrm{~Hz}, 2 \mathrm{H}), 2.28(\mathrm{~s}, 3 \mathrm{H}) ;{ }^{13} \mathbf{C} \mathbf{N M R}\left(75 \mathrm{MHz}, \mathrm{CDCl}_{3}\right): \delta_{\mathrm{C}}$ $(\mathrm{ppm})=190.6,158.9,143.9,137.2,135.8,134.8,134.3,132.0,129.6,128.7,128.4,127.7$, 127.6, 122.1, 120.2, 118.5, 110.7, 87.5, 81.2, 70.3, 49.6, 36.7, 21.4; HRMS-ESI (m/z): calcd. for $\mathrm{C}_{27} \mathrm{H}_{25} \mathrm{NO}_{4} \mathrm{SNa}[\mathrm{M}+\mathrm{Na}]^{+} 482.1397$, found 482.1394 .

1k: (E)-N-(3-(4-(benzyloxy)-2-formylphenyl)prop-2-yn-1-yl)- $N$-(but-2-en-1-yl)-4methylbenzenesulfonamide

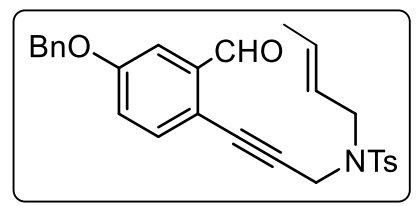

Yellow oil, (881 mg, yield 62\%), (silica gel, $n$-hexane/EtOAc $=$ 8:1); ${ }^{1} \mathbf{H}$ NMR $\left(300 \mathrm{MHz}, \mathrm{CDCl}_{3}\right): \delta_{\mathrm{H}}(\mathrm{ppm})=9.85(\mathrm{~s}, 1 \mathrm{H}), 7.75(\mathrm{~d}$, $J=8.19 \mathrm{~Hz}, 2 \mathrm{H}), 7.44-7.36(\mathrm{~m}, 6 \mathrm{H}), 7.21(\mathrm{~d}, J=8.43 \mathrm{~Hz}, 2 \mathrm{H})$, $7.17-7.09(\mathrm{~m}, 2 \mathrm{H}), 5.81-5.65(\mathrm{~m}, 1 \mathrm{H}), 5.51-5.37(\mathrm{~m}, 1 \mathrm{H}), 5.11(\mathrm{~s}, 2 \mathrm{H}), 4.36(\mathrm{~s}, 2 \mathrm{H}), 3.83$ $(\mathrm{d}, J=6.67 \mathrm{~Hz}, 2 \mathrm{H}), 2.26(\mathrm{~s}, 3 \mathrm{H}), 1.72(\mathrm{~d}, J=6.45 \mathrm{~Hz}, 3 \mathrm{H}) ;{ }^{13} \mathbf{C} \mathbf{N M R}\left(75 \mathrm{MHz}, \mathrm{CDCl}_{3}\right): \delta_{\mathrm{C}}$ $(\mathrm{ppm})=190.7,158.9,143.7,137.2,135.9,135.8,134.8,131.8,129.6,128.7,128.4,127.7$, 127.6, 124.6, 122.1, 118.7, 110.6, 87.7, 81.1, 70.3, 48.9, 36.4, 21.4, 17.8; Mass-ESI (m/z): calcd. for $\mathrm{C}_{28} \mathrm{H}_{27} \mathrm{NO}_{4} \mathrm{SK}[\mathrm{M}+\mathrm{K}]^{+}$512.1298, found 512.1293; Anal calcd: C, 71.01; H, 5.75; N, 2.96; S, 6.77. found: C, 71.03; H, 5.76; N, 3.00; S, 6.80 .

11: $N$-(3-(2-formylphenyl)prop-2-yn-1-yl)-4-methyl- $N$-(2-methylallyl)benzenesulfonamide

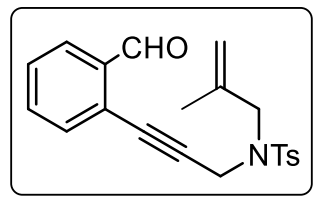

Yellow oil, (716 mg, yield 65\%), (silica gel, $n$-hexane/EtOAc $=7: 1) ;{ }^{1} \mathbf{H}$ NMR $\left(300 \mathrm{MHz}, \mathrm{CDCl}_{3}\right): \delta_{\mathrm{H}}(\mathrm{ppm})=9.88(\mathrm{~s}, 1 \mathrm{H}), 7.84(\mathrm{dd}, J=7.8,1.2$ $\mathrm{Hz}, 1 \mathrm{H}), 7.76(\mathrm{~d}, J=8.3 \mathrm{~Hz}, 2 \mathrm{H}), 7.49(\mathrm{td}, J=7.5,1.6 \mathrm{~Hz}, 1 \mathrm{H}), 7.41(\mathrm{t}$, $J=7.5 \mathrm{~Hz}, 1 \mathrm{H}), 7.20(\mathrm{~d}, J=7.8 \mathrm{~Hz}, 3 \mathrm{H}), 5.04-5.00(\mathrm{~m}, 2 \mathrm{H}), 4.34(\mathrm{~s}, 2 \mathrm{H}), 3.82(\mathrm{~s}, 2 \mathrm{H}), 2.25$ $(\mathrm{s}, 3 \mathrm{H}), 1.82(\mathrm{~s}, 3 \mathrm{H}) ;{ }^{13} \mathbf{C} \mathbf{N M R}\left(75 \mathrm{MHz}, \mathrm{CDCl}_{3}\right): \delta_{\mathrm{C}}(\mathrm{ppm})=190.7,143.8,139.2,135.9$, 135.8, 133.6, 133.4, 129.6, 128.8, 127.6, 127.1, 125.7, 115.8, 89.0, 81.4, 53.1, 36.3, 21.4, 19.7; Mass-ESI (m/z): calcd. for $\mathrm{C}_{21} \mathrm{H}_{22} \mathrm{NO}_{3} \mathrm{~S}[\mathrm{M}+\mathrm{H}]^{+}$368.1320, found 368.1315; Anal calcd: $\mathrm{C}$, 68.64; H, 5.76; N, 3.81; S, 8.72. found: C, 68.69; H, 5.79; N, 3.82; S, 8.75. 
1m: (Z)- $N$-(But-2-en-1-yl)- $N$-(3-(2-formylphenyl)prop-2-yn-1-yl)-4-methylbenzene sulfonamide

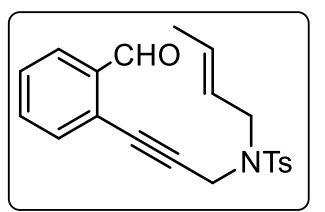

Yellow oil, (705 mg, yield 64\%), (silica gel, $n$-hexane/EtOAc $=7: 1$ ); ${ }^{1} \mathbf{H}$ NMR $\left(400 \mathrm{MHz}, \mathrm{CDCl}_{3}\right): \delta_{\mathrm{H}}(\mathrm{ppm})=9.90(\mathrm{~s}, 1 \mathrm{H}), 7.84(\mathrm{dd}, J=7.8,1.4$ $\mathrm{Hz}, 1 \mathrm{H}), 7.75(\mathrm{~d}, J=8.2 \mathrm{~Hz}, 2 \mathrm{H}), 7.50(\mathrm{td}, J=7.6,1.5 \mathrm{~Hz}, 1 \mathrm{H}), 7.42(\mathrm{t}, J$ $=7.6 \mathrm{~Hz}, 1 \mathrm{H}), 7.23(\mathrm{~d}, J=7.3 \mathrm{~Hz}, 1 \mathrm{H}), 7.20(\mathrm{~d}, J=8.1 \mathrm{~Hz}, 2 \mathrm{H}), 5.79-$ $5.69(\mathrm{~m}, 1 \mathrm{H}), 5.49-5.40(\mathrm{~m}, 1 \mathrm{H}), 4.37(\mathrm{~s}, 2 \mathrm{H}), 3.84(\mathrm{~d}, J=6.8 \mathrm{~Hz}, 2 \mathrm{H}), 2.25(\mathrm{~s}, 3 \mathrm{H}), 1.72(\mathrm{~d}$, $J=6.3 \mathrm{~Hz}, 3 \mathrm{H}) ;{ }^{13} \mathbf{C} \mathbf{N M R}\left(100 \mathrm{MHz}, \mathrm{CDCl}_{3}\right): \delta_{\mathrm{C}}(\mathrm{ppm})=190.8,143.8,135.8,135.7,133.6$, 133.4, 131.9, 129.6, 128.8, 127.7, 127.1, 125.8, 124.5, 89.3, 81.3, 48.9, 36.4, 21.4, 17.8. HRMS-ESI (m/z): calcd. for $\mathrm{C}_{42} \mathrm{H}_{42} \mathrm{~N}_{2} \mathrm{O}_{6} \mathrm{~S}_{2} \mathrm{Na}[2 \mathrm{M}+\mathrm{Na}]^{+}$757.2376, found 757.2374.

1n: $N$-cinnamyl- $N$-(3-(2-formylphenyl)prop-2-yn-1-yl)-4-methylbenzenesulfonamide

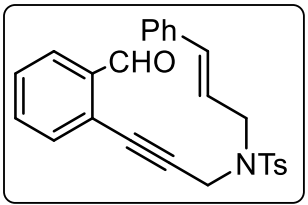

Yellow oil, (773 mg, yield 60\%), (silica gel, $n$-hexane/EtOAc $=8: 1) ;{ }^{1} \mathbf{H}$ NMR $\left(300 \mathrm{MHz}, \mathrm{CDCl}_{3}\right): \delta_{\mathrm{H}}(\mathrm{ppm})=9.95(\mathrm{~s}, 1 \mathrm{H}), 7.86(\mathrm{dd}, J=7.7,1.5$ $\mathrm{Hz}, 1 \mathrm{H}), 7.80(\mathrm{~d}, J=8.3 \mathrm{~Hz}, 2 \mathrm{H}), 7.54-7.48(\mathrm{~m}, 1 \mathrm{H}), 7.46-7.40(\mathrm{~m}$, 1H), $7.39-7.30(\mathrm{~m}, 4 \mathrm{H}), 7.29-7.21(\mathrm{~m}, 4 \mathrm{H}), 6.63(\mathrm{~d}, J=15.8 \mathrm{~Hz}, 1 \mathrm{H}), 6.23-6.12(\mathrm{~m}, 1 \mathrm{H})$, $4.44(\mathrm{~s}, 2 \mathrm{H}), 4.09$ (d, $J=6.8 \mathrm{~Hz}, 2 \mathrm{H}), 2.29(\mathrm{~s}, 3 \mathrm{H}) ;{ }^{13} \mathbf{C ~ N M R}\left(75 \mathrm{MHz}, \mathrm{CDCl}_{3}\right): \delta_{\mathrm{C}}(\mathrm{ppm})=$ $190.7,144.0,136.0,135.9,135.1,133.6,133.5,129.7,128.9,128.7,128.2,127.7,127.2$, 126.59, 125.6, 122.9, 89.2, 81.5, 49.2, 36.8, 21.4; Mass-ESI (m/z): calcd. for $\mathrm{C}_{26} \mathrm{H}_{24} \mathrm{NO}_{3} \mathrm{~S}$ $[\mathrm{M}+\mathrm{H}]^{+}$430.1477, found 430.1472; Anal calcd: C, 72.70; H, 5.40; N, 3.26; S, 7.46. found: C, 72.69; H, 5.38; N, 3.22; S, 7.44.

10: $N$-allyl- $N$-(3-(5-chloro-2-formylphenyl)prop-2-yn-1-yl)methanesulfonamide

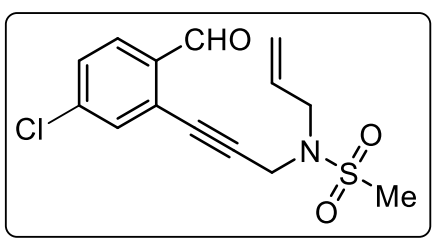

Yellow oil, (655 mg, yield 70\%), (silica gel, $n$-hexane/EtOAc $=$ $7: 1) ;{ }^{1} \mathbf{H}$ NMR $\left(400 \mathrm{MHz}, \mathrm{CDCl}_{3}\right): \delta_{\mathrm{H}}(\mathrm{ppm})=10.27(\mathrm{~s}, 1 \mathrm{H}), 7.78$ $(\mathrm{d}, J=8.4 \mathrm{~Hz}, 1 \mathrm{H}), 7.46(\mathrm{~d}, J=2.0 \mathrm{~Hz}, 1 \mathrm{H}), 7.41(\mathrm{dd}, J=8.3$, $1.7 \mathrm{~Hz}, 1 \mathrm{H}), 5.85-5.73(\mathrm{~m}, 1 \mathrm{H}), 5.38-5.31(\mathrm{~m}, 1 \mathrm{H}), 5.31-$ $5.26(\mathrm{~m}, 1 \mathrm{H}), 4.31(\mathrm{~s}, 1 \mathrm{H}), 3.93(\mathrm{~d}, J=6.4 \mathrm{~Hz}, 2 \mathrm{H}), 2.93(\mathrm{~s}, 3 \mathrm{H}) ;{ }^{13} \mathbf{C ~ N M R}\left(100 \mathrm{MHz}, \mathrm{CDCl}_{3}\right)$ : $\delta_{\mathrm{C}}(\mathrm{ppm})=189.5,140.4,134.6,133.5,131.83,130.0,129.8,126.0,120.3,90.9,80.7,49.57$, 38.8, 36.6; Mass-ESI (m/z): calcd. for $\mathrm{C}_{14} \mathrm{H}_{15} \mathrm{ClNO}_{3} \mathrm{~S}[\mathrm{M}+\mathrm{H}]^{+}$312.0465, found 312.0454; Anal calcd: C, 53.93; H, 4.53; N, 4.49; S, 10.28. found: C, 53.94; H, 4.54; N, 4.52; S, 10.30 . 


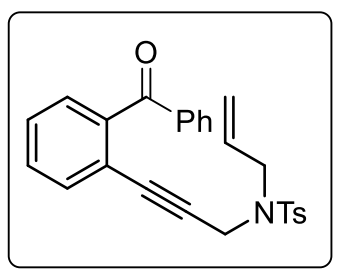

Yellow oil, (966 mg, yield 75\%), (silica gel, $n$-hexane/EtOAc $=7: 1) ;{ }^{1} \mathbf{H}$ NMR $\left(300 \mathrm{MHz}, \mathrm{CDCl}_{3}\right): \delta_{\mathrm{H}}(\mathrm{ppm})=7.76-7.62(\mathrm{~m}, 5 \mathrm{H}), 7.62-7.55$ (m, 1H), $7.47-7.42(\mathrm{~m}, 2 \mathrm{H}), 7.41-7.37(\mathrm{~m}, 3 \mathrm{H}), 7.23-7.18(\mathrm{~m}, 2 \mathrm{H})$, $5.72-5.53(\mathrm{~m}, 1 \mathrm{H}), 5.19-5.14(\mathrm{~m}, 1 \mathrm{H}), 5.14-5.06(\mathrm{~m}, 1 \mathrm{H}), 4.06(\mathrm{~s}$, 2H), $3.60-3.53(\mathrm{~m}, 2 \mathrm{H}), 2.32(\mathrm{~s}, 3 \mathrm{H}) ;{ }^{13} \mathbf{C} \mathbf{~ N M R}\left(75 \mathrm{MHz}, \mathrm{CDCl}_{3}\right): \delta_{\mathrm{C}}(\mathrm{ppm})=196.2,143.4$, $141.4,140.0,136.8,136.0,133.4,133.0,131.8,130.1,129.9,129.5,128.4,128.2$, 127.6, 120.7, 119.9, 87.3, 83.3, 48.9, 36.4, 21.4; Mass-ESI (m/z): calcd. for $\mathrm{C}_{26} \mathrm{H}_{24} \mathrm{NO}_{3} \mathrm{~S}[\mathrm{M}+\mathrm{H}]^{+} 430.1477$, found 430.1472; Anal calcd: C, 72.70; H, 5.40; N, 3.26; S, 7.46. found: C, 72.72; H, 5.41; N, $3.30 ; \mathrm{S}, 7.49$.

\section{3b: $N$-allyl- $N$-(3-(2-benzoylphenyl)prop-2-yn-1-yl)methanesulfonamide}

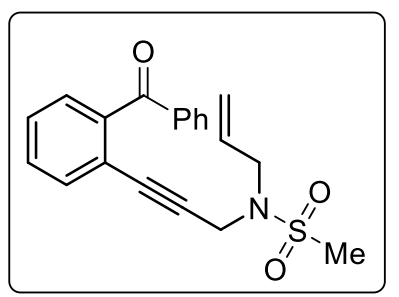

Yellow oil, (805 mg, yield 76\%), (silica gel, $n$-hexane/EtOAc $=7: 1$ ); ${ }^{1} \mathbf{H}$ NMR $\left(300 \mathrm{MHz}, \mathrm{CDCl}_{3}\right): \delta_{\mathrm{H}}(\mathrm{ppm})=7.84-7.80(\mathrm{~m}, 1 \mathrm{H}), 7.66-$ $7.59(\mathrm{~m}, 1 \mathrm{H}), 7.58-7.53(\mathrm{~m}, 1 \mathrm{H}), 7.52-7.37(\mathrm{~m}, 6 \mathrm{H}), 5.77-5.60$ (m, 1H), $5.27-5.09(\mathrm{~m}, 2 \mathrm{H}), 4.11(\mathrm{~s}, 2 \mathrm{H}), 3.64(\mathrm{~d}, J=6.4 \mathrm{~Hz}, 2 \mathrm{H})$,

$2.77(\mathrm{~s}, 3 \mathrm{H}) ;{ }^{13} \mathbf{C} \mathbf{N M R}\left(75 \mathrm{MHz}, \mathrm{CDCl}_{3}\right): \delta_{\mathrm{C}}(\mathrm{ppm})=196.4,141.8,136.6,133.8,133.0,131.9$, 130.3, 130.1, 128.7, 128.4, 127.9, 120.4, 120.0, 87.2, 83.8, 48.9, 38.0, 36.3; Mass-ESI (m/z): calcd. for $\mathrm{C}_{20} \mathrm{H}_{20} \mathrm{NO}_{3} \mathrm{~S}[\mathrm{M}+\mathrm{H}]^{+}$354.1164, founf 354.1157; Anal calcd: C, 67.97; H, 5.42; N, 3.96; S, 9.07. found: C, 68.00; H, 5.44; N, 3.99; S, 9.09.

\section{3c: (2-(3-(allyloxy)prop-1-yn-1-yl)phenyl)(phenyl)methanone}

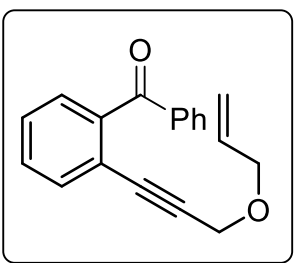

Yellow oil, (561 mg, yield 70\%), (silica gel, $n$-hexane/EtOAc $=8: 1) ;{ }^{1} \mathbf{H}$ NMR $\left(300 \mathrm{MHz}, \mathrm{CDCl}_{3}\right): \delta_{\mathrm{H}}(\mathrm{ppm})=7.85-7.78(\mathrm{~m}, 2 \mathrm{H}), 7.61-7.54$ $(\mathrm{m}, 2 \mathrm{H}), 7.50-7.42(\mathrm{~m}, 5 \mathrm{H}), 5.86-5.71(\mathrm{~m}, 1 \mathrm{H}), 5.26-5.11(\mathrm{~m}, 2 \mathrm{H})$, $4.12(\mathrm{~s}, 2 \mathrm{H}), 3.80(\mathrm{dt}, J=5.8,1.4 \mathrm{~Hz}, 2 \mathrm{H}) ;{ }^{13} \mathbf{C ~ N M R}\left(75 \mathrm{MHz}, \mathrm{CDCl}_{3}\right)$ : $\delta_{\mathrm{C}}(\mathrm{ppm})=196.8,141.8,137.1,133.9,133.2,133.0,130.2,130.1,128.4,128.3,128.2,121.2$, 117.8, 90.6, 84.0, 70.2, 57.5; Mass-ESI (m/z): calcd. for $\mathrm{C}_{19} \mathrm{H}_{17} \mathrm{O}_{2}[\mathrm{M}+\mathrm{H}]^{+}$277.1229, found 277.1221; Anal calcd: C, 82.58; H, 5.84. found: C, 82.60; H, 5.85. 


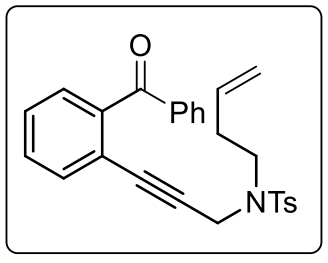

Yellow oil, (944 mg, yield 71\%), (silica gel, $n$-hexane/EtOAc $=7: 1) ;{ }^{1} \mathbf{H}$ NMR $\left(300 \mathrm{MHz}, \mathrm{CDCl}_{3}\right): \delta_{\mathrm{H}}(\mathrm{ppm})=7.73-7.68(\mathrm{~m}, 2 \mathrm{H}), 7.66(\mathrm{~d}, J=$ $8.3 \mathrm{~Hz}, 2 \mathrm{H}), 7.61-7.55(\mathrm{~m}, 1 \mathrm{H}), 7.45$ (d, $J=7.8 \mathrm{~Hz}, 2 \mathrm{H}), 7.42-7.39$ $(\mathrm{m}, 3 \mathrm{H}), 7.21(\mathrm{~d}, J=8.0 \mathrm{~Hz}, 2 \mathrm{H}), 7.18-7.12(\mathrm{~m}, 1 \mathrm{H}), 5.80-5.57(\mathrm{~m}$, 1H), $5.10-4.99$ (m, 2H), 4.09 (s, 2H), 3.00 (t, J= 7.2 Hz, 2H), 2.32 (s, 3H), $2.22-2.11$ (m, $2 \mathrm{H}) ;{ }^{13} \mathrm{C}$ NMR $\left(75 \mathrm{MHz}, \mathrm{CDCl}_{3}\right): \delta_{\mathrm{C}}(\mathrm{ppm})=196.2,143.4,141.5,136.9,135.8,134.6,133.3$, 132.9, 130.1, 129.9, 129.5, 128.4, 128.3, 128.6, 127.6, 120.7, 117.1, 87.4, 83.3, 45.5, 37.0, 32.2, 21.4; Mass-ESI (m/z): calcd. for $\mathrm{C}_{27} \mathrm{H}_{26} \mathrm{NO}_{3} \mathrm{~S}[\mathrm{M}+\mathrm{H}]^{+}$444.1633, found 444.1622; Anal calcd: C, 73.11; H, 5.68; N, 3.16; S, 7.23. found: C, 73.15; H, 5.70; N, 3.18; S, 7.24.

3e: $N$-allyl- $N$-(3-(2-benzoylphenyl)prop-2-yn-1-yl)methanesulfonamide

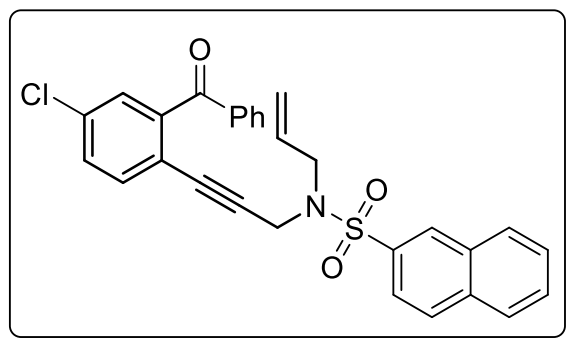

Yellow solid mp, $113-115^{\circ} \mathrm{C},(1 \mathrm{gr}$, yield $72 \%)$, (silica gel, $n$-hexane/EtOAc $=7: 1)$; ${ }^{1} \mathbf{H}$ NMR $\left(300 \mathrm{MHz}, \mathrm{CDCl}_{3}\right)$ : $\delta_{\mathrm{H}}(\mathrm{ppm})=8.43(\mathrm{~s}, 1 \mathrm{H}), 7.96(\mathrm{~d}, J=7.90 \mathrm{~Hz}, 1 \mathrm{H}), 7.92-$ $7.84(\mathrm{~m}, 2 \mathrm{H}), 7.78(\mathrm{~d}, J=8.7 \mathrm{~Hz}, 1 \mathrm{H}), 7.70-7.56(\mathrm{~m}$, $5 \mathrm{H}), 7.41(\mathrm{t}, J=7.6 \mathrm{~Hz}, 2 \mathrm{H}), 7.29(\mathrm{~d}, J=6.7 \mathrm{~Hz}, 1 \mathrm{H}), 7.12$ $(\mathrm{dd}, J=8.3,1.9 \mathrm{~Hz}, 1 \mathrm{H}), 6.55(\mathrm{~d}, J=8.3 \mathrm{~Hz}, 1 \mathrm{H}), 5.74-5.58(\mathrm{~m}, 1 \mathrm{H}), 5.18(\mathrm{~d}, J=7.5 \mathrm{~Hz}$, 1H), $5.13(\mathrm{~d}, J=14.7 \mathrm{~Hz}, 1 \mathrm{H}), 4.13(\mathrm{~s}, 2 \mathrm{H}), 3.66(\mathrm{~d}, J=6.3 \mathrm{~Hz}, 2 \mathrm{H}) ;{ }^{13} \mathbf{C}$ NMR $(75 \mathrm{MHz}$, $\left.\mathrm{CDCl}_{3}\right): \delta_{\mathrm{C}}(\mathrm{ppm})=194.6,142.7,136.2,136.0,134.9,134.3,133.9,133.7,132.2,131.7,130.0$, 129.9, 129.3, 129.1, 129.1, 128.7, 128.6, 128.0, 127.9, 127.4, 122.9, 120.1, 118.8, 88.1, 82.3, 49.1, 36.4; Mass-ESI (m/z): calcd. for $\mathrm{C}_{29} \mathrm{H}_{23} \mathrm{ClNO}_{3} \mathrm{~S}[\mathrm{M}+\mathrm{H}]^{+}$500.1087, found 500.1089; Anal calcd: C, 69.66; H, 4.44; N, 2.80; S, 6.41. found: C, 69.64; H, 4.43; N, 2.79; S, 6.38.

\section{3f: Dimethyl 2-allyl-2-(3-(2-benzoylphenyl)prop-2-yn-1-yl)malonate}

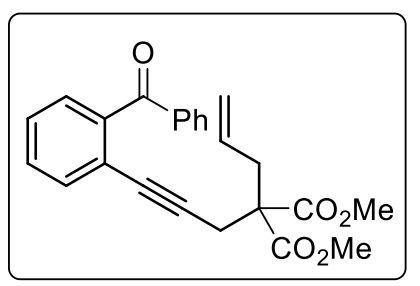

Yellow oil, $(832 \mathrm{mg}$, yield 72\%), (silica gel, $n$-hexane/EtOAc $=$ 6:1); ${ }^{1} \mathbf{H}$ NMR $\left(300 \mathrm{MHz}, \mathrm{CDCl}_{3}\right): \delta_{\mathrm{H}}(\mathrm{ppm})=7.85-7.78(\mathrm{~m}, 2 \mathrm{H})$, $7.61-7.54(\mathrm{~m}, 1 \mathrm{H}), 7.51-7.44(\mathrm{~m}, 3 \mathrm{H}), 7.44-7.36(\mathrm{~m}, 3 \mathrm{H}), 5.55$ $-5.38(\mathrm{~m}, 1 \mathrm{H}), 5.07-4.98(\mathrm{~m}, 2 \mathrm{H}), 3.67(\mathrm{~s}, 6 \mathrm{H}), 2.79(\mathrm{~s}, 2 \mathrm{H}), 2.54$ $(\mathrm{d}, J=7.4 \mathrm{~Hz}, 2 \mathrm{H}) ;{ }^{13} \mathbf{C} \mathbf{N M R}\left(75 \mathrm{MHz}, \mathrm{CDCl}_{3}\right): \delta_{\mathrm{C}}(\mathrm{ppm})=196.7,170.1,141.7,136.9,133.3$, $133.1,131.8,130.2,129.9,128.4,127.9,127.8,121.5,119.7,103.8,89.7,81.3,57.0,52.7$, 
36.5, 23.6; Mass-ESI (m/z): calcd. for $\mathrm{C}_{24} \mathrm{H}_{23} \mathrm{O}_{5}[\mathrm{M}+\mathrm{H}]^{+}$391.1545, found 391.1539; Anal calcd: C, 73.83; H, 5.68. found: C, 73.85; H, 5.71.

2a: (9S,10aS)-2-tosyl-1,2,3,9,10,10a-hexahydro-4H-3a,9-epoxybenzo[4,5]cyclohepta[1,2-c] pyrrol-4-one

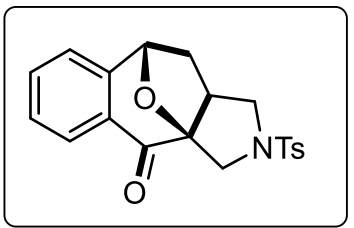

Colourless solid, mp 180-181 ${ }^{\circ} \mathrm{C},(52 \mathrm{mg}$, yield 70\%), (silica gel, $n$ hexane/EtOAc $=3: 1) ;{ }^{1} \mathbf{H}$ NMR $\left(400 \mathrm{MHz}, \mathrm{CDCl}_{3}\right): \delta_{\mathrm{H}}(\mathrm{ppm})=7.97$ $(\mathrm{d}, J=7.7 \mathrm{~Hz}, 1 \mathrm{H}), 7.75(\mathrm{~d}, J=8.1 \mathrm{~Hz}, 2 \mathrm{H}), 7.54(\mathrm{td}, J=7.5,1.3 \mathrm{~Hz}$, $1 \mathrm{H}), 7.40(\mathrm{~d}, J=7.5 \mathrm{~Hz}, 1 \mathrm{H}), 7.36(\mathrm{~d}, J=8.1 \mathrm{~Hz}, 2 \mathrm{H}), 7.20(\mathrm{~d}, J=7.6$ $\mathrm{Hz}, 1 \mathrm{H}), 5.40$ (d, $J=6.2 \mathrm{~Hz}, 1 \mathrm{H},), 4.01$ (d, $J=11.5 \mathrm{~Hz}, 1 \mathrm{H},), 3.53$ (d, $J=11.6 \mathrm{~Hz}, 1 \mathrm{H}), 3.48$ $(\mathrm{dd}, J=10.0,8.5 \mathrm{~Hz}, 1 \mathrm{H}), 3.26(\mathrm{dd}, J=10.1,5.5 \mathrm{~Hz}, 1 \mathrm{H}), 2.67-2.58(\mathrm{~m}, 1 \mathrm{H}), 2.45(\mathrm{~s}, 3 \mathrm{H})$, $2.26-2.13(\mathrm{~m}, 2 \mathrm{H}) ;{ }^{13} \mathbf{C}$ NMR $\left(100 \mathrm{MHz}, \mathrm{CDCl}_{3}\right): \delta_{\mathrm{C}}(\mathrm{ppm})=191.7,146.3,143.9,134.3$, $132.3,129.8,128.3,128.0,127.4,126.5,124.0,95.4$, 80.4, 53.9, 51.4, 44.1, 38.1, 21.6; HRMSESI (m/z): calcd. for $\mathrm{C}_{40} \mathrm{H}_{38} \mathrm{~N}_{2} \mathrm{O}_{8} \mathrm{~S}_{2} \mathrm{Na}[2 \mathrm{M}+\mathrm{Na}]^{+} 761.1962$, found 761.1959.

2b: (9S,10aS)-2-(methylsulfonyl)-1,2,3,9,10,10a-hexahydro-4H-3a,9-epoxybenzo[4,5] cyclohepta[1,2-c]pyrrol-4-one

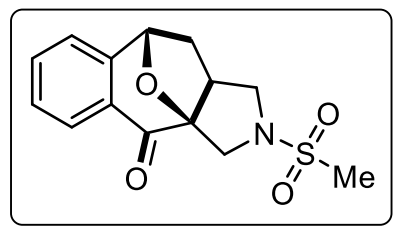

Colourless solid, mp $174-175{ }^{\circ} \mathrm{C},(42 \mathrm{mg}$, yield $72 \%)$, (silica gel, $n$ hexane/EtOAc $=3: 1) ;{ }^{1} \mathbf{H}$ NMR $\left(500 \mathrm{MHz}, \mathrm{CDCl}_{3}\right): \delta_{\mathrm{H}}(\mathrm{ppm})=8.04$ $(\mathrm{dd}, J=7.8,1.4 \mathrm{~Hz}, 1 \mathrm{H}), 7.58(\mathrm{td}, J=7.5,1.4 \mathrm{~Hz}, 1 \mathrm{H}), 7.44(\mathrm{td}, J=$ 7.6, $1.2 \mathrm{~Hz}, 1 \mathrm{H}), 7.25(\mathrm{dd}, J=7.6,1.2 \mathrm{~Hz}, 1 \mathrm{H}), 5.51(\mathrm{~d}, J=6.6 \mathrm{~Hz}$, $1 \mathrm{H}), 4.36(\mathrm{~d}, J=12.3 \mathrm{~Hz}, 1 \mathrm{H}), 3.87(\mathrm{dd}, J=10.8,8.6 \mathrm{~Hz}, 1 \mathrm{H}), 3.63(\mathrm{~d}, J=12.3 \mathrm{~Hz}, 1 \mathrm{H}), 3.38$ $(\mathrm{dd}, J=10.8,6.6 \mathrm{~Hz}, 1 \mathrm{H}), 2.93(\mathrm{~s}, 3 \mathrm{H}), 2.86-2.79(\mathrm{~m}, 1 \mathrm{H}), 2.34-2.28(\mathrm{~m}, 1 \mathrm{H}), 2.25-2.20$ $(\mathrm{m}, 1 \mathrm{H}) ;{ }^{13} \mathbf{C} \mathbf{N M R}\left(125 \mathrm{MHz}, \mathrm{CDCl}_{3}\right): \delta_{\mathrm{C}}(\mathrm{ppm})=192.1,146.5,134.6,128.5,128.1,127.6$, 124.1, 96.4, 80.7, 54.6, 51.4, 45.0, 38.3, 35.9; HRMS-ESI (m/z): calcd. for $\mathrm{C}_{28} \mathrm{H}_{30} \mathrm{~N}_{2} \mathrm{O}_{8} \mathrm{~S}_{2} \mathrm{Na}$ $[2 \mathrm{M}+\mathrm{Na}]^{+} 609.1336$, found 609.1333 .

2c: benzyl $(9 S, 10 a S)-4-0 x o-4,9,10,10 a-t e t r a h y d r o-1 H-3 a, 9-e p o x y b e n z o[4,5]$ cyclohepta[1,2-c] pyrrole-2(3H)-carboxylate

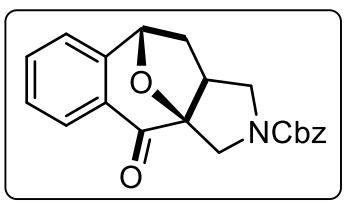

Colourless solid, mp 142-144 ${ }^{\circ} \mathrm{C},(48 \mathrm{mg}$, yield 69\%), (silica gel, $n$ hexane/EtOAc $=3: 1) ;{ }^{1} \mathbf{H}$ NMR $\left(300 \mathrm{MHz}, \mathrm{CDCl}_{3}\right): \delta_{\mathrm{H}}(\mathrm{ppm})=8.04$ $(\mathrm{dd}, J=7.7,1.4 \mathrm{~Hz}, 1 \mathrm{H}), 7.57(\mathrm{td}, J=7.5,1.4 \mathrm{~Hz}, 1 \mathrm{H}), 7.46-7.30(\mathrm{~m}$, $6 \mathrm{H}), 7.24(\mathrm{~d}, J=7.7 \mathrm{~Hz}, 1 \mathrm{H}), 5.48(\mathrm{~d}, J=6.7 \mathrm{~Hz}, 1 \mathrm{H}), 5.17(\mathrm{~s}, 2 \mathrm{H})$, 
4.55 - $4.44(\mathrm{~m}, 1 \mathrm{H}), 4.13-4.00(\mathrm{~m}, 1 \mathrm{H}), 3.84-3.71(\mathrm{~m}, 1 \mathrm{H}), 3.52-3.40(\mathrm{~m}, 1 \mathrm{H}), 2.87-2.75$ $(\mathrm{m}, 1 \mathrm{H}), 2.38-2.27(\mathrm{~m}, 1 \mathrm{H}), 2.25-2.12(\mathrm{~m}, 1 \mathrm{H}) ;{ }^{13} \mathbf{C} \mathbf{N M R}\left(75 \mathrm{MHz}, \mathrm{CDCl}_{3}\right): \delta_{\mathrm{C}}(\mathrm{ppm})=$ 192.3, 147.0, 136.7, 134.3, 128.5, 128.3, 128.0, 127.9, 127.5, 123.8, 96.8, 80.2, 67.0, 53.6, 49.5, 43.2, 39.0; HRMS-ESI (m/z): calcd. for $\mathrm{C}_{21} \mathrm{H}_{20} \mathrm{NO}_{4}[\mathrm{M}+\mathrm{H}]^{+} 350.1387$, found 350.1382.

2d: (9S,10aS)-2-(naphthalen-2-ylsulfonyl)-1,2,3,9,10,10a-hexahydro-4H-3a,9-epoxybenzo [4,5] cyclohepta[1,2-c]pyrrol-4-one

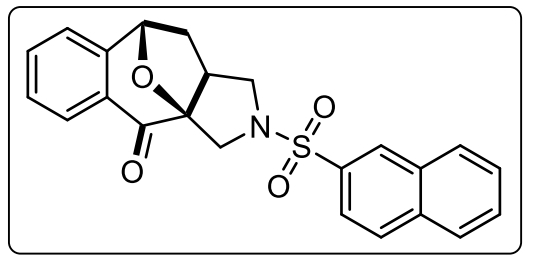

Colourless solid, mp $180-182{ }^{\circ} \mathrm{C}$, (66 mg, yield $\left.81 \%\right)$, (silica gel, $n$-hexane/EtOAc $=3: 1) ;{ }^{1} \mathbf{H}$ NMR $(400 \mathrm{MHz}$, $\left.\mathrm{CDCl}_{3}\right): \delta_{\mathrm{H}}(\mathrm{ppm})=8.45(\mathrm{~d}, J=1.3 \mathrm{~Hz}, 1 \mathrm{H}), 8.01(\mathrm{~d}, J=$ $8.6 \mathrm{~Hz}, 2 \mathrm{H}), 7.97-7.92(\mathrm{~m}, 2 \mathrm{H}), 7.88(\mathrm{dd}, J=8.6,1.8 \mathrm{~Hz}$, 1H), $7.69-7.61(\mathrm{~m}, 2 \mathrm{H}), 7.52(\mathrm{td}, J=7.5,1.3 \mathrm{~Hz}, 1 \mathrm{H}), 7.38(\mathrm{td}, J=7.7,0.9 \mathrm{~Hz}, 1 \mathrm{H}), 7.17$ (d, $J=7.6 \mathrm{~Hz}, 1 \mathrm{H}), 5.35(\mathrm{~d}, J=6.3 \mathrm{~Hz}, 1 \mathrm{H}), 4.18(\mathrm{~d}, J=11.6 \mathrm{~Hz}, 1 \mathrm{H}), 3.64(\mathrm{~d}, J=10.0 \mathrm{~Hz}, 1 \mathrm{H})$, $3.62(\mathrm{~d}, J=11.1 \mathrm{~Hz}, 1 \mathrm{H}), 3.31(\mathrm{dd}, J=10.1,5.8 \mathrm{~Hz}, 1 \mathrm{H}), 2.66-2.58(\mathrm{~m}, 1 \mathrm{H}), 2.22-2.09(\mathrm{~m}$, $2 \mathrm{H}) ;{ }^{13} \mathrm{C} \mathrm{NMR}\left(100 \mathrm{MHz}, \mathrm{CDCl}_{3}\right): \delta_{\mathrm{C}}(\mathrm{ppm})=191.6,146.3,135.0,134.3,132.8,132.2,129.4$, $128.9,128.3,128.1,128.0,127.6,127.4,124.0$, 123.2, 95.5, 80.4, 54.1, 51.5, 44.2, 38.0; HRMS-ESI (m/z): calcd. for $\mathrm{C}_{23} \mathrm{H}_{20} \mathrm{NO}_{4} \mathrm{~S}[\mathrm{M}+\mathrm{H}]^{+} 406.1108$, found 406.1111 .

2e: dimethyl (9S,10aR)-4-oxo-4,9,10,10a-tetrahydro-1H-3a,9-epoxybenzo[f]azulene-2,2(3H)dicarboxylate

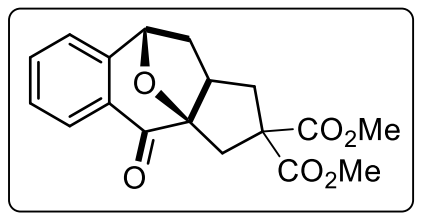

Colourless solid, mp 137-138 ${ }^{\circ} \mathrm{C}$, (43 mg, yield 65\%), (silica gel, $n$-hexane/EtOAc $=2: 1) ;{ }^{1} \mathbf{H}$ NMR $\left(500 \mathrm{MHz}, \mathrm{CDCl}_{3}\right): \delta_{\mathrm{H}}(\mathrm{ppm})=$ $8.00(\mathrm{dd}, J=7.8,1.4 \mathrm{~Hz}, 1 \mathrm{H}), 7.51(\mathrm{td}, J=7.5,1.4 \mathrm{~Hz}, 1 \mathrm{H}), 7.38$ $(\mathrm{td}, J=7.6,1.2 \mathrm{~Hz}, 1 \mathrm{H}), 7.17(\mathrm{dd}, J=7.5,1.2 \mathrm{~Hz}, 1 \mathrm{H}), 5.31(\mathrm{~d}, J=$ $6.8 \mathrm{~Hz}, 1 \mathrm{H}), 3.77$ (s, 3H), 3.75 (s, 3H), 3.28 (d, J=14.7 Hz, 1H), $2.74-2.66$ (m, 2H), 2.56 $2.51(\mathrm{~m}, 1 \mathrm{H}), 2.44-2.37(\mathrm{~m}, 2 \mathrm{H}), 2.15(\mathrm{dd}, J=12.1,9.2 \mathrm{~Hz}, 1 \mathrm{H}) ;{ }^{13} \mathbf{C}$ NMR $(125 \mathrm{MHz}$, $\left.\mathrm{CDCl}_{3}\right): \delta_{\mathrm{C}}(\mathrm{ppm})=193.7,171.5,171.5,146.9,134.0,128.4,128.0,127.2,124.0,97.0,80.1$, 62.0, 53.3, 52.8, 43.9, 39.7, 39.6, 37.7; HRMS-ESI (m/z): calcd. for $\mathrm{C}_{36} \mathrm{H}_{36} \mathrm{O}_{12} \mathrm{Na}[2 \mathrm{M}+\mathrm{Na}]^{+}$ 683.2099,found 683.2096. 
2f: $(9 \mathrm{~S}, 10 \mathrm{a} S)-1,9,10,10 \mathrm{a}-t$ tetrahydro-3H,4H-3a,9-epoxybenzo[4,5]cyclohepta[1,2-c]furan4-one

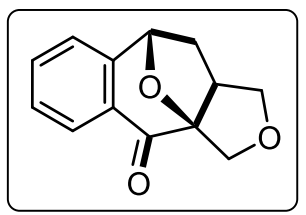

Colourless solid, mp 123-125 ${ }^{\circ} \mathrm{C}$, (29 mg, yield 67\%), (silica gel, $n$ hexane/EtOAc $=4: 1) ;{ }^{1} \mathbf{H}$ NMR $\left(400 \mathrm{MHz}, \mathrm{CDCl}_{3}\right): \delta_{\mathrm{H}}(\mathrm{ppm})=8.03(\mathrm{~d}, J$ $=7.7 \mathrm{~Hz}, 1 \mathrm{H}), 7.55(\mathrm{td}, J=7.5,1.3 \mathrm{~Hz}, 1 \mathrm{H}), 7.42(\mathrm{td}, J=7.6,1.0 \mathrm{~Hz}, 1 \mathrm{H})$, $7.24(\mathrm{~d}, J=7.6 \mathrm{~Hz}, 1 \mathrm{H}), 5.51(\mathrm{~d}, J=6.2 \mathrm{~Hz}, 1 \mathrm{H}), 4.55(\mathrm{~d}, J=10.5 \mathrm{~Hz}$, $1 \mathrm{H}), 4.16-4.10(\mathrm{~m}, 1 \mathrm{H}), 4.01(\mathrm{~d}, J=10.5 \mathrm{~Hz}, 1 \mathrm{H}), 3.81(\mathrm{dd}, J=8.9,5.6 \mathrm{~Hz}, 1 \mathrm{H}), 2.84-2.73$ (m, 1H), $2.29-2.15(\mathrm{~m}, 2 \mathrm{H}) ;{ }^{13} \mathbf{C} \mathbf{N M R}\left(100 \mathrm{MHz}, \mathrm{CDCl}_{3}\right): \delta_{\mathrm{C}}(\mathrm{ppm})=192.9,146.6,134.1$, 128.9, 128.2, 127.2, 124.2, 97.8, 81.4, 74.4, 70.9, 47.0, 37.6; HRMS-APCI (m/z): calcd. for $\mathrm{C}_{13} \mathrm{H}_{12} \mathrm{O}_{3}[\mathrm{M}+\mathrm{H}]^{+}$217.0859, found 217.0860.

\section{2g: (4aR,6S)-2-tosyl-2,3,4,4a,5,6-hexahydro-6,11a-epoxybenzo[5,6]cyclohepta[1,2-c]} pyridin-11(1H)-one

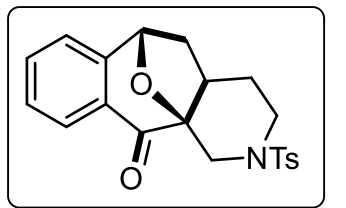

Colourless solid, mp 182-184 ${ }^{\circ} \mathrm{C},(54 \mathrm{mg}$, yield $71 \%)$, (silica gel, $n$ hexane/EtOAc $=3: 1) ;{ }^{1} \mathbf{H}$ NMR $\left(400 \mathrm{MHz}, \mathrm{CDCl}_{3}\right): \delta_{\mathrm{H}}(\mathrm{ppm})=7.95(\mathrm{~d}$, $J=7.7 \mathrm{~Hz}, 1 \mathrm{H}), 7.71(\mathrm{~d}, J=8.3 \mathrm{~Hz}, 2 \mathrm{H}), 7.54(\mathrm{td}, J=7.5,1.4 \mathrm{~Hz}, 1 \mathrm{H})$, $7.40(\mathrm{~d}, J=7.6 \mathrm{~Hz}, 1 \mathrm{H}), 7.33(\mathrm{~d}, J=8.1 \mathrm{~Hz}, 2 \mathrm{H}), 7.21(\mathrm{~d}, J=7.5 \mathrm{~Hz}, 1 \mathrm{H}), 5.25(\mathrm{~d}, J=6.6 \mathrm{~Hz}$, 1H), $3.99(\mathrm{dd}, J=13.7,1.4 \mathrm{~Hz}, 1 \mathrm{H}), 3.71-3.62(\mathrm{~m}, 1 \mathrm{H}), 3.60(\mathrm{~d}, J=13.7 \mathrm{~Hz}, 1 \mathrm{H}), 2.75-$ $2.65(\mathrm{~m}, 1 \mathrm{H}), 2.45(\mathrm{~s}, 3 \mathrm{H}), 2.17-1.97(\mathrm{~m}, 4 \mathrm{H}), 1.78-1.65(\mathrm{~m}, 1 \mathrm{H}) ;{ }^{13} \mathbf{C}$ NMR $(100 \mathrm{MHz}$, $\left.\mathrm{CDCl}_{3}\right): \delta_{\mathrm{C}}(\mathrm{ppm})=195.3,148.3,143.4,134.3,134.1,129.6,128.0,128.0,127.7,127.6,123.2$, 85.6, 76.0, 45.0, 43.0, 40.0, 34.4, 29.1, 21.6; HRMS-ESI (m/z): calcd. for $\mathrm{C}_{21} \mathrm{H}_{22} \mathrm{NO}_{4} \mathrm{~S}$ $[\mathrm{M}+\mathrm{H}]^{+}$384.1264, found 384.1264.

2h: (4aR,6S)-8-methoxy-2-tosyl-2,3,4,4a,5,6-hexahydro-6,11a-epoxybenzo[5,6] cyclohepta[1,2-c]pyridin-11(1H)-one

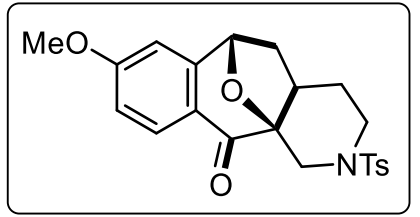

Colourless solid, mp 196-197 ${ }^{\circ} \mathrm{C}$, (68 mg, yield 82\%), (silica gel, $n$-hexane/EtOAc $=3: 1) ;{ }^{1} \mathbf{H}$ NMR $\left(400 \mathrm{MHz}, \mathrm{CDCl}_{3}\right): \delta_{\mathrm{H}}(\mathrm{ppm})=$ $7.90(\mathrm{~d}, J=8.6 \mathrm{~Hz}, 1 \mathrm{H}), 7.69(\mathrm{~d}, J=8.2 \mathrm{~Hz}, 2 \mathrm{H}), 7.32(\mathrm{~d}, J=8.1$ $\mathrm{Hz}, 2 \mathrm{H}), 6.85(\mathrm{dd}, J=8.6,2.5 \mathrm{~Hz}, 1 \mathrm{H}), 6.66(\mathrm{~d}, J=2.4 \mathrm{~Hz}, 1 \mathrm{H}), 5.18(\mathrm{~d}, J=6.9 \mathrm{~Hz}, 1 \mathrm{H}), 3.97$ (d, $J=13.7 \mathrm{~Hz}, 1 \mathrm{H}), 3.87$ (s, 3H), $3.69-3.62(\mathrm{~m}, 1 \mathrm{H}), 3.58$ (d, $J=13.7 \mathrm{~Hz}, 1 \mathrm{H}), 2.69-2.60$ (m, 1H), $2.43(\mathrm{~s}, 3 \mathrm{H}), 2.12-1.94(\mathrm{~m}, 4 \mathrm{H}), 1.73-1.63(\mathrm{~m}, 1 \mathrm{H}) ;{ }^{13} \mathbf{C} \mathbf{N M R}\left(100 \mathrm{MHz}, \mathrm{CDCl}_{3}\right)$ : $\delta_{\mathrm{C}}(\mathrm{ppm})=194.1,164.4,151.0,143.4,134.3,130.3,129.7,127.8,121.3,113.8,107.9,85.5$, 
76.2, 55.7, 45.2, 43.2, 40.3, 34.8, 29.4, 21.6; HRMS-ESI (m/z): calcd. for $\mathrm{C}_{22} \mathrm{H}_{24} \mathrm{NO}_{5} \mathrm{~S}$ $[\mathrm{M}+\mathrm{H}]^{+}$414.1370, found 414.1373.

\section{2i: (9S,10aS)-7-methoxy-2-tosyl-1,2,3,9,10,10a-hexahydro-4H-3a,9-epoxybenzo}

$[4,5]$ cyclohepta[1,2-c]pyrrol-4-one

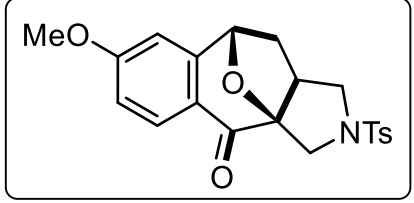

Colourless solid, mp 191-193 ${ }^{\circ} \mathrm{C}$, (62 mg, yield 78\%), (silica gel, $n$-hexane/EtOAc $=3: 1) ;{ }^{1} \mathbf{H}$ NMR $\left(400 \mathrm{MHz}, \mathrm{CDCl}_{3}\right): \delta_{\mathrm{H}}(\mathrm{ppm})=$ $7.93(\mathrm{~d}, J=8.7 \mathrm{~Hz}, 1 \mathrm{H}), 7.75(\mathrm{~d}, J=8.2 \mathrm{~Hz}, 2 \mathrm{H}), 7.35(\mathrm{~d}, J=8.1$

$\mathrm{Hz}, 2 \mathrm{H}), 6.87(\mathrm{dd}, J=8.7,2.5 \mathrm{~Hz}, 1 \mathrm{H}), 6.64(\mathrm{~d}, J=2.4 \mathrm{~Hz}, 1 \mathrm{H}), 5.33(\mathrm{~d}, J=6.4 \mathrm{~Hz}, 1 \mathrm{H}), 4.05$ $(\mathrm{d}, J=11.5 \mathrm{~Hz}, 1 \mathrm{H}), 3.86(\mathrm{~s}, 3 \mathrm{H}), 3.53-3.48(\mathrm{~m}, 2 \mathrm{H}), 3.23(\mathrm{dd}, J=10.1,5.8 \mathrm{~Hz}, 1 \mathrm{H}), 2.64-$ $2.56(\mathrm{~m}, 1 \mathrm{H}), 2.44(\mathrm{~s}, 3 \mathrm{H}), 2.23-2.09(\mathrm{~m}, 2 \mathrm{H}) ;{ }^{13} \mathbf{C} \mathbf{~ N M R}\left(100 \mathrm{MHz}, \mathrm{CDCl}_{3}\right): \delta_{\mathrm{C}}(\mathrm{ppm})=$ 190.8, 164.4, 148.9, 143.9, 130.0, 129.8, 128.1, 121.5, 114.0, 108.8, 95.6, 80.6, 55.7, 54.1, 51.4, 44.6, 38.3, 21.6; HRMS-ESI (m/z): calcd. for $\mathrm{C}_{21} \mathrm{H}_{22} \mathrm{NO}_{5} \mathrm{~S}[\mathrm{M}+\mathrm{H}]^{+} 400.1213$, found 400.1216.

2j: (9S,10aS)-7-(benzyloxy)-2-tosyl-1,2,3,9,10,10a-hexahydro-4H-3a,9-epoxybenzo[4,5] cyclohepta[1,2-c]pyrrol-4-one

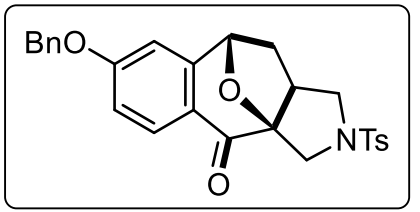

Colourless solid, mp 195-197 ${ }^{\circ} \mathrm{C}$, (79 mg, yield 83\%), (silica gel, $n$-hexane/EtOAc $=4: 1) ;{ }^{1} \mathbf{H}$ NMR $\left(400 \mathrm{MHz}, \mathrm{CDCl}_{3}\right): \delta_{\mathrm{H}}(\mathrm{ppm})=$ $7.93(\mathrm{~d}, J=8.7 \mathrm{~Hz}, 1 \mathrm{H}), 7.75(\mathrm{~d}, J=8.2 \mathrm{~Hz}, 2 \mathrm{H}), 7.43-7.38(\mathrm{~m}$, 4H), $7.37-7.32(\mathrm{~m}, 3 \mathrm{H}), 6.94(\mathrm{dd}, J=8.7,2.4 \mathrm{~Hz}, 1 \mathrm{H}), 6.72(\mathrm{~d}, J=2.4 \mathrm{~Hz}, 1 \mathrm{H}), 5.32(\mathrm{~d}, J=$ $6.3 \mathrm{~Hz}, 1 \mathrm{H}), 5.11(\mathrm{~s}, 2 \mathrm{H}), 4.05(\mathrm{~d}, J=11.5 \mathrm{~Hz}, 1 \mathrm{H}), 3.54-3.48(\mathrm{~m}, 2 \mathrm{H}), 3.23(\mathrm{dd}, J=10.1$, $5.8 \mathrm{~Hz}, 1 \mathrm{H}), 2.64-2.56(\mathrm{~m}, 1 \mathrm{H}), 2.44(\mathrm{~s}, 3 \mathrm{H}), 2.22-2.08(\mathrm{~m}, 2 \mathrm{H}) ;{ }^{13} \mathrm{C}$ NMR $(100 \mathrm{MHz}$, $\left.\mathrm{CDCl}_{3}\right): \delta_{\mathrm{C}}(\mathrm{ppm})=190.7,163.5,148.9,143.9,135.8,132.8,130.0,129.8,128.8,128.5,128.1$, 127.5, 121.7, 114.7, 109.7, 95.6, 80.6, 70.4, 54.1, 51.4, 44.6, 38.2, 21.6; HRMS-ESI (m/z): calcd. for $\mathrm{C}_{27} \mathrm{H}_{26} \mathrm{NO}_{5} \mathrm{~S}[\mathrm{M}+\mathrm{H}]^{+} 476.1526$, found 476.1527 .

2k: (9S,10R,10aS)-7-(benzyloxy)-10-methyl-2-tosyl-1,2,3,9,10,10a-hexahydro-4H-3a,9epoxybenzo[4,5]cyclohepta[1,2-c]pyrrol-4-one

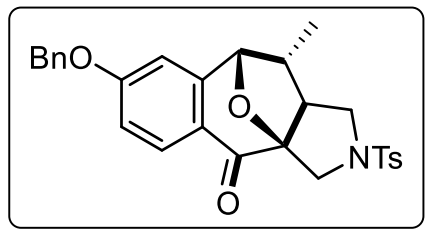

Colourless solid, mp 185-187 ${ }^{\circ} \mathrm{C}$, (78 mg, yield 80\%), (silica gel, $n$-hexane/EtOAc $=4: 1) ;{ }^{1} \mathbf{H}$ NMR $\left(300 \mathrm{MHz}, \mathrm{CDCl}_{3}\right): \delta_{\mathrm{H}}(\mathrm{ppm})=$ $7.99(\mathrm{~d}, J=8.66 \mathrm{~Hz}, 1 \mathrm{H}), 7.77(\mathrm{~d}, J=8.19 \mathrm{~Hz}, 2 \mathrm{H}), 7.47-7.36$ 
$(\mathrm{m}, 7 \mathrm{H}), 7.01(\mathrm{dd}, J=8.66,2.36 \mathrm{~Hz}, 1 \mathrm{H}), 6.71(\mathrm{~d}, J=2.32 \mathrm{~Hz}, 1 \mathrm{H}), 5.21-5.11(\mathrm{~m}, 3 \mathrm{H}), 3.74$ $(\mathrm{d}, J=11.23 \mathrm{~Hz}, 1 \mathrm{H}), 3.60(\mathrm{~d}, J=11.22 \mathrm{~Hz}, 1 \mathrm{H}), 3.45(\mathrm{dd}, J=9.95,3.45 \mathrm{~Hz}, 1 \mathrm{H}), 3.23$ (t, $J$ $=9.15 \mathrm{~Hz}, 1 \mathrm{H}), 2.67-2.55(\mathrm{~m}, 1 \mathrm{H}), 2.49(\mathrm{~s}, 3 \mathrm{H}), 2.12-2.03(\mathrm{~m}, 1 \mathrm{H}), 0.82(\mathrm{~d}, J=6.99 \mathrm{~Hz}$, $3 \mathrm{H}) ;{ }^{13} \mathrm{C}$ NMR $\left(75 \mathrm{MHz}, \mathrm{CDCl}_{3}\right): \delta_{\mathrm{C}}(\mathrm{ppm})=191.1,162.8,145.1,143.9,135.7,132.0,129.8$, 129.8, 128.8, 128.4, 128.1, 127.5, 122.4, 114.7, 112.0, 94.9, 84.1, 70.4, 52.5, 52.1, 51.4, 44.4, 21.6, 15.2; Mass-ESI (m/z): calcd. for $\mathrm{C}_{28} \mathrm{H}_{28} \mathrm{NO}_{5} \mathrm{~S}[\mathrm{M}+\mathrm{H}]^{+}$490.1688, found 490.1681; Anal calcd: C, 68.69; H, 5.56; N, 2.86; S, 6.55. found: C, 68.70; H, 5.58; N, 2.87; S, 6.57.

\section{1: $(9 S, 10 a S)-10 a-m e t h y l-2-t o s y l-1,2,3,9,10,10 a-h e x a h y d r o-4 H-3 a, 9-e p o x y b e n z o[4,5]$ cyclohepta[1,2-c]pyrrol-4-one}

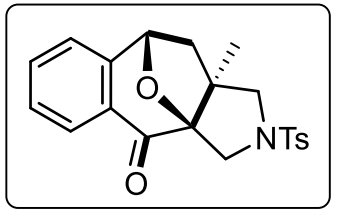

Yellow oil, (53 mg, yield 69\%), (silica gel, $n$-hexane/EtOAc $=3: 1$ ); ${ }^{1} \mathbf{H}$ NMR $\left(300 \mathrm{MHz}, \mathrm{CDCl}_{3}\right): \delta_{\mathrm{H}}(\mathrm{ppm})=8.02(\mathrm{~d}, J=7.1 \mathrm{~Hz}, 1 \mathrm{H}), 7.77(\mathrm{~d}$, $J=8.2 \mathrm{~Hz}, 2 \mathrm{H}), 7.56(\mathrm{td}, J=7.5,1.4 \mathrm{~Hz}, 1 \mathrm{H}), 7.43(\mathrm{~d}, J=7.6 \mathrm{~Hz}, 1 \mathrm{H})$, $7.38(\mathrm{~d}, J=8.2 \mathrm{~Hz}, 2 \mathrm{H}), 7.19(\mathrm{~d}, J=7.5 \mathrm{~Hz}, 1 \mathrm{H}), 5.30(\mathrm{~d}, J=7.2 \mathrm{~Hz}, 1 \mathrm{H}), 4.37(\mathrm{~d}, J=11.6$ $\mathrm{Hz}, 1 \mathrm{H}), 3.48$ (d, $J=9.4 \mathrm{~Hz}, 1 \mathrm{H}), 3.42$ (d, $J=11.6 \mathrm{~Hz}, 1 \mathrm{H}), 3.32$ (d, $J=9.5 \mathrm{~Hz}, 1 \mathrm{H}), 2.53$ (dd, $J=12.5,7.3 \mathrm{~Hz}, 1 \mathrm{H}), 2.47(\mathrm{~s}, 3 \mathrm{H}), 1.72(\mathrm{~d}, J=12.5 \mathrm{~Hz}, 1 \mathrm{H}), 0.95(\mathrm{~s}, 3 \mathrm{H}) ;{ }^{13} \mathbf{C} \mathbf{N M R}(75 \mathrm{MHz}$, $\left.\mathrm{CDCl}_{3}\right): \delta_{\mathrm{C}}(\mathrm{ppm})=191.5,147.1,143.8,134.4,133.1,129.8,128.3,128.3,127.8,127.2,123.6$, 97.8, 79.1, 62.4, 51.1, 50.7, 43.7, 22.0, 21.6; Mass-ESI (m/z): calcd. for $\mathrm{C}_{21} \mathrm{H}_{22} \mathrm{NO}_{4} \mathrm{~S}[\mathrm{M}+\mathrm{H}]^{+}$ 384.1270, found 384.1269; Anal calcd: C, 65.78; H, 5.52; N, 3.65; S, 8.36. found: C, 65.81; H, 5.54; N, 3.69; S, 8.38.

\section{2m: (9S,10R,10aS)-10-methyl-2-tosyl-1,2,3,9,10,10a-hexahydro-4H-3a,9-epoxybenzo} [4,5]cyclohepta[1,2-c]pyrrol-4-one

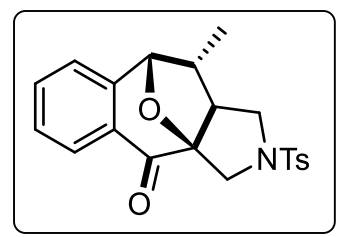

Colourless solid, mp 172-173 ${ }^{\circ} \mathrm{C},(56 \mathrm{mg}$, yield 73\%), (silica gel, $n$ hexane/EtOAc $=3: 1) ;{ }^{1} \mathbf{H}$ NMR $\left(400 \mathrm{MHz}, \mathrm{CDCl}_{3}\right): \delta_{\mathrm{H}}(\mathrm{ppm})=8.00(\mathrm{~d}$, $J=7.7 \mathrm{~Hz}, 1 \mathrm{H}), 7.74(\mathrm{~d}, J=8.0 \mathrm{~Hz}, 2 \mathrm{H}), 7.55(\mathrm{td}, J=7.5,1.4 \mathrm{~Hz}, 1 \mathrm{H})$, $7.42(\mathrm{t}, J=7.5 \mathrm{~Hz}, 1 \mathrm{H}), 7.37(\mathrm{~d}, J=8.0 \mathrm{~Hz}, 2 \mathrm{H}), 7.16(\mathrm{~d}, J=7.6 \mathrm{~Hz}$, 1H), $5.18(\mathrm{~d}, J=6.1 \mathrm{~Hz}, 1 \mathrm{H}), 3.69$ (d, $J=11.2 \mathrm{~Hz}, 1 \mathrm{H}), 3.59$ (d, $J=11.2 \mathrm{~Hz}, 1 \mathrm{H}), 3.45(\mathrm{dd}, J$ $=10.0,3.3 \mathrm{~Hz}, 1 \mathrm{H}), 3.22-3.15(\mathrm{~m}, 1 \mathrm{H}), 2.66-2.56(\mathrm{~m}, 1 \mathrm{H}), 2.46(\mathrm{~s}, 3 \mathrm{H}), 2.09-2.02(\mathrm{~m}$, $1 \mathrm{H}), 0.82(\mathrm{~d}, J=7.0 \mathrm{~Hz}, 3 \mathrm{H}) ;{ }^{13} \mathbf{C} \mathbf{N M R}\left(100 \mathrm{MHz}, \mathrm{CDCl}_{3}\right): \delta_{\mathrm{C}}(\mathrm{ppm})=192.0,144.0,142.7$, 
133.6, 131.8, 129.8, 129.0, 128.4, 128.1, 127.4, 126.1, 94.9, 83.9, 52.4, 51.7, 51.4, 44.3, 21.6, 15.2; HRMS-ESI (m/z): calcd. for $\mathrm{C}_{42} \mathrm{H}_{42} \mathrm{~N}_{2} \mathrm{O}_{8} \mathrm{~S}_{2} \mathrm{Na}[2 \mathrm{M}+\mathrm{Na}]^{+} 789.2275$, found 789.2271.

2n: (9S,10S,10aS)-10-phenyl-2-tosyl-1,2,3,9,10,10a-hexahydro-4H-3a,9-epoxybenzo[4,5] cyclohepta[1,2-c]pyrrol-4-one

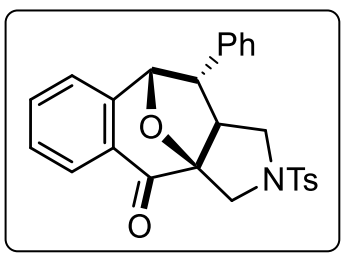

Yellow foam solid, (53 mg, yield 59\%), (silica gel, $n$-hexane/EtOAc $=$ $4: 1) ;{ }^{1} \mathbf{H}$ NMR $\left(300 \mathrm{MHz}, \mathrm{CDCl}_{3}\right): \delta_{\mathrm{H}}(\mathrm{ppm})=8.08(\mathrm{~d}, J=7.6 \mathrm{~Hz}, 1 \mathrm{H})$, $7.78(\mathrm{~d}, J=8.2 \mathrm{~Hz}, 2 \mathrm{H}), 7.43-7.38(\mathrm{~m}, 3 \mathrm{H}), 7.35-7.29(\mathrm{~m}, 2 \mathrm{H}), 7.18$ $-7.13(\mathrm{~m}, 2 \mathrm{H}), 6.69-6.63(\mathrm{~m}, 2 \mathrm{H}), 6.55(\mathrm{~d}, J=7.5 \mathrm{~Hz}, 1 \mathrm{H}), 5.37$ (d, $J$ $=6.1 \mathrm{~Hz}, 1 \mathrm{H}), 3.87-3.81(\mathrm{~m}, 1 \mathrm{H}), 3.77(\mathrm{~d}, J=2.0 \mathrm{~Hz}, 2 \mathrm{H}), 3.58(\mathrm{dd}, J=10.2,2.9 \mathrm{~Hz}, 1 \mathrm{H})$, $3.20(\mathrm{dd}, J=10.1,8.3 \mathrm{~Hz}, 1 \mathrm{H}), 2.85-2.78(\mathrm{~m}, 1 \mathrm{H}), 2.50(\mathrm{~s}, 3 \mathrm{H}) ;{ }^{13} \mathbf{C} \mathbf{N M R}\left(75 \mathrm{MHz}, \mathrm{CDCl}_{3}\right)$ : $\delta_{\mathrm{C}}(\mathrm{ppm})=191.8,144.1,142.2,135.4,133.3,131.8,129.8,129.2,128.5,128.4,128.2,128.2$, 127.6, 127.0, 126.9, 95.1, 84.9, 56.6, 52.5, 51.5, 50.5, 21.6; Mass-ESI (m/z): calcd. for $\mathrm{C}_{26} \mathrm{H}_{24} \mathrm{NO}_{4} \mathrm{~S}[\mathrm{M}+\mathrm{H}]^{+}$446.1426, found 446.1429; Anal calcd: C, 70.09; H, 5.20; N, 3.14; S, 7.20. found: C, 70.10; H, 5.21; N, 3.16; S, 7.23.

2o: $(9 S, 10 a S)-6$-chloro-2-(methylsulfonyl)-1,2,3,9,10,10a-hexahydro-4H-3a,9-epoxybenzo $[4,5]$ cyclohepta[1,2-c]pyrrol-4-one

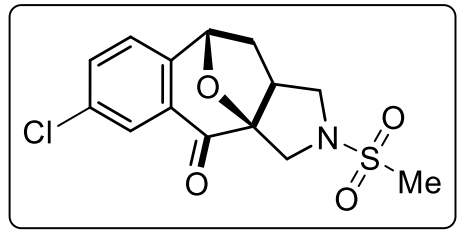

Yellow solid, mp 192-194 ${ }^{\circ} \mathrm{C},(56 \mathrm{mg}$, yield 85\%), (silica gel, $n$ hexane/EtOAc $=3: 1) ;{ }^{1} \mathbf{H}$ NMR $\left(400 \mathrm{MHz}, \mathrm{CDCl}_{3}\right): \delta_{\mathrm{H}}(\mathrm{ppm})=$ $8.00(\mathrm{~d}, J=2.1 \mathrm{~Hz}, 1 \mathrm{H}), 7.55(\mathrm{dd}, J=8.1,2.2 \mathrm{~Hz}, 1 \mathrm{H}), 7.23(\mathrm{~d}$, $J=8.1 \mathrm{~Hz}, 1 \mathrm{H}), 5.52(\mathrm{~d}, J=6.5 \mathrm{~Hz}, 1 \mathrm{H}), 4.33(\mathrm{~d}, J=12.3 \mathrm{~Hz}$ 1H), $3.91-3.84(\mathrm{~m}, 1 \mathrm{H}), 3.64(\mathrm{~d}, J=12.3 \mathrm{~Hz}, 1 \mathrm{H}), 3.43-3.37(\mathrm{~m}, 1 \mathrm{H}), 2.94(\mathrm{~s}, 3 \mathrm{H}), 2.88-$ $2.79(\mathrm{~m}, 1 \mathrm{H}), 2.38-2.29(\mathrm{~m}, 1 \mathrm{H}), 2.26-2.19(\mathrm{~m}, 1 \mathrm{H}) ;{ }^{13} \mathbf{C} \mathbf{~ N M R}\left(100 \mathrm{MHz}, \mathrm{CDCl}_{3}\right): \delta_{\mathrm{C}}$ $(\mathrm{ppm})=190.9,144.7,134.7,134.3,129.4,127.4,125.7,96.0,80.2,54.3,51.2,44.7,38.1,35.8$; Mass-ESI (m/z): calcd. for $\mathrm{C}_{14} \mathrm{H}_{15} \mathrm{ClNO}_{4} \mathrm{~S}[\mathrm{M}+\mathrm{H}]^{+}$328.0410, found 328.0395; Anal calcd: $\mathrm{C}$, 51.30; H, 4.31; N, 4.27; S, 9.78. found: C, 51.29; H, 4.28; N, 4.26; S, 9.77.

4a: (2-benzoylphenyl)((5S)-3-tosyl-3-azabicyclo[3.1.0]hexan-1-yl)methanone

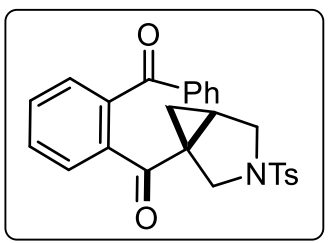

Colourless foam solid, (61 mg, yield 68\%), (silica gel, $n$-hexane/EtOAc $=3: 1) ;{ }^{1} \mathbf{H}$ NMR $\left(300 \mathrm{MHz}, \mathrm{CDCl}_{3}\right): \delta_{\mathrm{H}}(\mathrm{ppm})=7.72-7.68(\mathrm{~m}, 2 \mathrm{H})$, $7.65(\mathrm{~d}, J=8.3 \mathrm{~Hz}, 2 \mathrm{H}), 7.62-7.56(\mathrm{~m}, 4 \mathrm{H}), 7.49-7.43(\mathrm{~m}, 2 \mathrm{H}), 7.40$ 
(d, $J=7.7 \mathrm{~Hz}, 1 \mathrm{H}), 7.33(\mathrm{~d}, J=8.1 \mathrm{~Hz}, 2 \mathrm{H}), 3.77(\mathrm{~d}, J=9.5 \mathrm{~Hz}, 1 \mathrm{H}), 3.57$ (d, $J=9.5 \mathrm{~Hz}, 1 \mathrm{H})$, $3.41(\mathrm{~d}, J=9.5 \mathrm{~Hz}, 1 \mathrm{H}), 3.19(\mathrm{dd}, J=9.5,4.0 \mathrm{~Hz}, 1 \mathrm{H}), 2.46(\mathrm{~s}, 3 \mathrm{H}), 2.13-2.06(\mathrm{~m}, 1 \mathrm{H}), 1.58$ $-1.52(\mathrm{~m}, 1 \mathrm{H}), 1.29(\mathrm{~d}, J=1.6 \mathrm{~Hz}, 1 \mathrm{H}), 1.22(\mathrm{t}, J=5.2 \mathrm{~Hz}, 1 \mathrm{H}) ;{ }^{13} \mathbf{C} \mathbf{N M R}\left(75 \mathrm{MHz}, \mathrm{CDCl}_{3}\right)$ : $\delta_{\mathrm{C}}(\mathrm{ppm})=202.5,196.1,143.8,140.8,137.7,136.9,133.3,133.1,131.1,130.6,129.9,129.8$, 128.4, 127.5, 126.8, 126.4, 50.1, 49.0, 38.2, 29.3, 21.6, 19.8; Mass-ESI (m/z): calcd. for $\mathrm{C}_{26} \mathrm{H}_{24} \mathrm{NO}_{4} \mathrm{~S}[\mathrm{M}+\mathrm{H}]^{+}$446.1426, found 446.1423; Anal calcd: $\mathrm{C}$, 70.09; H, 5.20; N, 3.14; S, 7.20. found: C, 70.11; H, 5.21; N, 3.17; S, 7.22.

4b: (2-benzoylphenyl)((5S)-3-(methylsulfonyl)-3-azabicyclo[3.1.0]hexan-1-yl)methanone

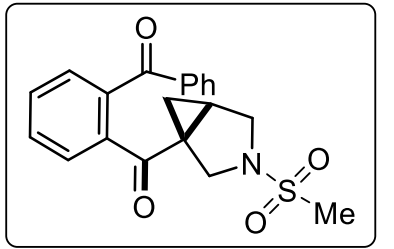

Yellow oil, (46 mg, yield 62\%), (silica gel, $n$-hexane/EtOAc = 3:1); ${ }^{1} \mathbf{H}$ NMR $\left(300 \mathrm{MHz}, \mathrm{CDCl}_{3}\right): \delta_{\mathrm{H}}(\mathrm{ppm})=77.85-7.79(\mathrm{~m}, 2 \mathrm{H}), 7.66$ - $7.57(\mathrm{~m}, 4 \mathrm{H}), 7.55-7.49(\mathrm{~m}, 3 \mathrm{H}), 3.77(\mathrm{~d}, J=9.4 \mathrm{~Hz}, 1 \mathrm{H}), 3.67-$ $3.59(\mathrm{~m}, 2 \mathrm{H}), 3.47(\mathrm{dd}, J=9.5,4.0 \mathrm{~Hz}, 1 \mathrm{H}), 2.84(\mathrm{~s}, 3 \mathrm{H}), 2.31-2.22(\mathrm{~m}, 1 \mathrm{H}), 1.77-1.68(\mathrm{~m}$, $1 \mathrm{H}), 1.41(\mathrm{t}, J=5.2 \mathrm{~Hz}, 1 \mathrm{H}) ;{ }^{13} \mathbf{C} \mathbf{~ N M R}\left(75 \mathrm{MHz}, \mathrm{CDCl}_{3}\right): \delta_{\mathrm{C}}(\mathrm{ppm})=202.9,196.3,141.2$, 137.5, 136.8, 133.3, 131.3, 130.4, 130.1, 129.6, 128.6, 126.7, 50.2, 49.0, 38.4, 35.5, 29.8, 19.7; Mass-ESI (m/z): calcd. for $\mathrm{C}_{20} \mathrm{H}_{20} \mathrm{NO}_{4} \mathrm{~S}[\mathrm{M}+\mathrm{H}]^{+}$370.1113, found 370.1107; Anal calcd: $\mathrm{C}$, 65.02; H, 5.18; N, 3.79; S, 8.68. found: C, 65.05; H, 5.20; N, 3.82 S, 8.70.

4c: (2-((5S)-3-oxabicyclo[3.1.0]hexane-1-carbonyl)phenyl)(phenyl)methanone

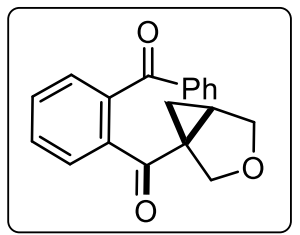

Colourless oil, (32 mg, yield 55\%), (silica gel, $n$-hexane/EtOAc $=4: 1) ;{ }^{1} \mathbf{H}$ NMR $\left(300 \mathrm{MHz}, \mathrm{CDCl}_{3}\right): \delta_{\mathrm{H}}(\mathrm{ppm})=7.81(\mathrm{~d}, J=7.1 \mathrm{~Hz}, 1 \mathrm{H}), 7.65-7.57$ $(\mathrm{m}, 4 \mathrm{H}), 7.56-7.47(\mathrm{~m}, 4 \mathrm{H}), 4.04-3.97(\mathrm{~m}, 2 \mathrm{H}), 3.87-3.80(\mathrm{~m}, 2 \mathrm{H})$, $2.23-2.16(\mathrm{~m}, 1 \mathrm{H}), 1.66-1.61(\mathrm{~m}, 1 \mathrm{H}), 1.24-1.17(\mathrm{~m}, 1 \mathrm{H}) ;{ }^{13} \mathbf{C} \mathbf{N M R}\left(75 \mathrm{MHz}, \mathrm{CDCl}_{3}\right)$ : $\delta_{\mathrm{C}}(\mathrm{ppm})=203.1,196.4,141.4,137.9,137.1,133.1,130.9,130.2,130.0,129.7,128.5,126.8$, 69.7, 68.7, 39.9, 32.4, 18.7; Mass-ESI (m/z): calcd. for $\mathrm{C}_{19} \mathrm{H}_{17} \mathrm{O}_{3}[\mathrm{M}+\mathrm{H}]^{+}$293.1178, found 293.1178; Anal calcd: C, 78.06; H, 5.52. found: C, 78.07; H, 5.55.

4d: (2-benzoylphenyl)(3-tosyl-3-azabicyclo[4.1.0]heptan-1-yl)methanone

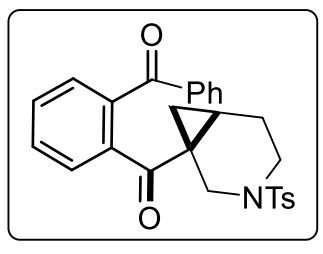

Yellow oil, (55 mg, yield 60\%), (silica gel, $n$-hexane/EtOAc $=3: 1) ;{ }^{1} \mathbf{H}$ NMR $\left(300 \mathrm{MHz}, \mathrm{CDCl}_{3}\right): \delta_{\mathrm{H}}(\mathrm{ppm})=7.79(\mathrm{~d}, J=7.2 \mathrm{~Hz}, 2 \mathrm{H}), 7.63-$ $7.53(\mathrm{~m}, 6 \mathrm{H}), 7.49(\mathrm{t}, J=7.6 \mathrm{~Hz}, 2 \mathrm{H}), 7.33-7.28(\mathrm{~m}, 3 \mathrm{H}), 3.74(\mathrm{~d}, J=$ $12.2 \mathrm{~Hz}, 1 \mathrm{H}), 3.43(\mathrm{~d}, J=12.1 \mathrm{~Hz}, 1 \mathrm{H}), 2.90(\mathrm{t}, J=6.3 \mathrm{~Hz}, 2 \mathrm{H}), 2.45(\mathrm{~s}$, 4H), $2.16(\mathrm{dd}, J=13.8,6.8 \mathrm{~Hz}, 1 \mathrm{H}), 2.01-1.85(\mathrm{~m}, 1 \mathrm{H}), 1.73-1.67(\mathrm{~m}, 1 \mathrm{H}), 1.61(\mathrm{dd}, J=$ 
9.7, 4.3 Hz, $1 \mathrm{H}), 1.02(\mathrm{dd}, J=6.8,4.3 \mathrm{~Hz}, 1 \mathrm{H}) ;{ }^{13} \mathbf{C ~ N M R}\left(75 \mathrm{MHz}, \mathrm{CDCl}_{3}\right): \delta_{\mathrm{C}}(\mathrm{ppm})=204.9$, 196.3, 143.6, 140.3 137.8, 136.9, 133.5, 133.0, 131.2, 130.2, 130.1, 129.7, 129.4, 128.5, 127.8, 127.5, 46.8, 41.8, 30.6, 22.8, 21.8, 21.5, 20.7; Mass-ESI (m/z): calcd. for $\mathrm{C}_{27} \mathrm{H}_{26} \mathrm{NO}_{4} \mathrm{~S}[\mathrm{M}+\mathrm{H}]^{+}$ 460.1583, found 460.1574; Anal calcd: C, 70.57; H, 5.48; N, 3.05; S, 6.98. found: C, 70.59; $\mathrm{H}, 5.50 ; \mathrm{N}, 3.06 ; \mathrm{S}, 7.00$.

4e: (2-benzoyl-4-chlorophenyl)((5S)-3-(naphthalen-2-ylsulfonyl)-3-azabicyclo[3.1.0] hexan-1-yl) methanone

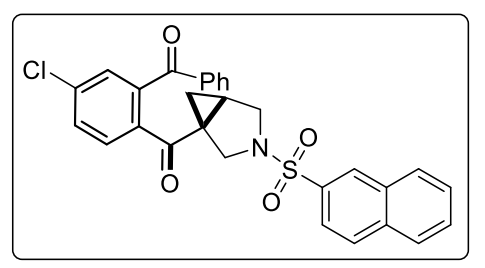

Yellow foam solid, (73 $\mathrm{mg}$, yield 71\%), (silica gel, $n$ hexane/EtOAc $=3: 1) ;{ }^{1} \mathbf{H}$ NMR $\left(300 \mathrm{MHz}, \mathrm{CDCl}_{3}\right): \delta_{\mathrm{H}}(\mathrm{ppm})=$ $8.36(\mathrm{~s}, 1 \mathrm{H}), 8.02-7.95(\mathrm{~m}, 3 \mathrm{H}), 7.77(\mathrm{dd}, J=8.64,1.75 \mathrm{~Hz}$, $1 \mathrm{H}), 7.69(\mathrm{t}, J=7.54 \mathrm{~Hz}, 2 \mathrm{H}), 7.60(\mathrm{~d}, J=7.08 \mathrm{~Hz}, 2 \mathrm{H}), 7.55-$ $7.49(\mathrm{~m}, 3 \mathrm{H}), 7.41-7.35(\mathrm{~m}, 2 \mathrm{H}), 7.34-7.28(\mathrm{~m}, 2 \mathrm{H}), 3.83(\mathrm{~d}, J=9.47 \mathrm{~Hz}, 1 \mathrm{H}), 3.67$ (d, $J=$ $9.56 \mathrm{~Hz}, 1 \mathrm{H}), 3.44(\mathrm{~d}, J=9.46 \mathrm{~Hz}, 1 \mathrm{H}), 3.28(\mathrm{dd}, J=9.53,3.95 \mathrm{~Hz}, 1 \mathrm{H}), 2.13-2.05(\mathrm{~m}, 1 \mathrm{H})$, $1.59-1.51(\mathrm{~m}, 1 \mathrm{H}), 1.29-1.23(\mathrm{~m}, 1 \mathrm{H}) ;{ }^{13} \mathbf{C} \mathbf{~ N M R}\left(75 \mathrm{MHz}, \mathrm{CDCl}_{3}\right): \delta_{\mathrm{C}}(\mathrm{ppm})=201.2$, 194.7, 139.6, 138.7, 136.2 136.2, 135.0, 133.5, 133.3, 132.2 130.9, 130.1, 129.8, 129.4, 129.3, 129.0, 128.8, 128.5, 128.1, 128.0, 127.7, 122.7, 50.1, 49.0, 38.3, 29.6, 19.9; Mass-ESI (m/z): calcd. for $\mathrm{C}_{29} \mathrm{H}_{23} \mathrm{ClNO}_{4} \mathrm{~S}[\mathrm{M}+\mathrm{H}]^{+}$516.1036, found 516.1038; Anal calcd: C, 67.50; H, 4.30; N, 2.71; S, 6.21. found: C, 67.53; H, 4.31; N, 2.72; S, 6.25. 


\section{References}

(1) Zhou, Y.; Nikbakht, A.; Bauer, F.; Breit, B. Chem.sci, 2019, 10, 4805-4810.

(2) Yan, F.; Liang, H.; Song, J.; Cui, J.; Liu, Q.; Liu, S.; Wang, P.; Dong, Y.; Liu, H. Org. lett, 2017, 19, 86-89.

(3) Kammerer, C.; Prestat, G.; Gaillard, T.; Madec, D.; Poli, G. Org. lett, 2008, 10, 405-408.

(4) Cabrera-Lobera, N.; Quirós, M. T.; Brennessel, W. W.; Neidig, M. L.; Buñuel, E.; Cárdenas, D. J. Org. lett, 2019, 21, 6552-6556.

(5) Gaussian 09, Revision B.01, Frisch, M. J.; Trucks, G. W.; Schlegel, H. B.; Scuseria, G. E.; Robb, M. A.; Cheeseman, J. R.; Scalmani, G.; Barone, V.; Mennucci, B.; Petersson, G. A.; Nakatsuji, H.; Caricato, M.; Li, X.; Hratchian, H. P.; Izmaylov, A. F.; Bloino, J.; Zheng, G.; Sonnenberg, J. L.; Hada, M.; Ehara, M.; Toyota, K.; Fukuda, R.; Hasegawa, J.; Ishida, M.; Nakajima, T.; Honda, Y.; Kitao, O.; Nakai, H.; Vreven, T.; Montgomery, J. A., Jr.; Peralta, J. E.; Ogliaro, F.; Bearpark, M.; Heyd, J. J.; Brothers, E.; Kudin, K. N.; Staroverov, V. N.; Kobayashi, R.; Normand, J.; Raghavachari, K.; Rendell, A.; Burant, J. C.; Iyengar, S. S.; Tomasi, J.; Cossi, M.; Rega, N.; Millam, J. M.; Klene, M.; Knox, J. E.; Cross, J. B.; Bakken, V.; Adamo, C.; Jaramillo, J.; Gomperts, R.; Stratmann, R. E.; Yazyev, O.; Austin, A. J.; Cammi, R.; Pomelli, C.; Ochterski, J. W.; Martin, R. L.; Morokuma, K.; Zakrzewski, V. G.; Voth, G. A.; Salvador, P.; Dannenberg, J. J.; Dapprich, S.; Daniels, A. D.; Farkas, Ö.; Foresman, J. B.; Ortiz, J. V.; Cioslowski, J.; Fox, D. J. Gaussian, Inc., Wallingford CT, 2009.

(6) CYLview, 1.0b; Legault, C. Y., Université de Sherbrooke, 2009 (http://www.cylview.org).

(7) (a) Becke, A. D. J. Chem. Phys.1993, 98, 5648-5652 (b) Becke, A. D. Phy. Rev. A. 1988, 38, 3098-3100 (c) Lee, C.; Yang, W.; Parr, R. G. Phys. Rev. B. 1988, 37, 785-789 (d) Hariharan, P. C.; Pople, J. A. Theor. Chim. Acta. 1973, 28, 213-222.

(8) Ebrahimi, A.; Rezazadeh, M.; Khosravi, H.; Rostami, A.; Al-Harrasi, A. ChemPlusChem, 2020, $85,1587-1595$.

(9) Funes-Ardoiz, I.; Nelson, D. J.; Maseras, F. Chem. Eur. J. 2017, 23, 16728-16733. 


\section{Z-matrices}

$1 \mathbf{b}$

C $\quad 3.76431100 \quad-1.75191500 \quad-0.04968600$

C $\quad 5.13376900 \quad-1.62096100 \quad 0.16394400$

C $\quad 5.70418800 \quad-0.35903200 \quad 0.34893100$

$\begin{array}{llll}\text { C } & 4.88594400 & 0.76469800 & 0.31492000\end{array}$

$\begin{array}{llll}\text { C } & 3.50509600 & 0.65808400 & 0.09553600\end{array}$

C $\quad 2.92179300 \quad-0.62473400 \quad-0.08898700$

$\begin{array}{llll}\mathrm{H} & 3.32379400 & -2.73337800 & -0.18847600\end{array}$

$\begin{array}{llll}\mathrm{H} & 5.75769500 & -2.50940000 & 0.18857100\end{array}$

$\mathrm{H} \quad 6.77121600 \quad-0.25659800 \quad 0.51801300$

$\begin{array}{llll}\mathrm{H} & 5.31450900 & 1.75380400 & 0.45795500\end{array}$

$\begin{array}{llll}\text { C } & 2.73263200 & 1.91685000 & 0.07009800\end{array}$

$\begin{array}{lllll}\mathrm{O} & 1.54006900 & 2.02951400 & -0.14466900\end{array}$

$\begin{array}{llll}\mathrm{H} & 3.35290200 & 2.81849300 & 0.27040200\end{array}$

$\begin{array}{llll}\text { C } & 1.52809700 & -0.82796800 & -0.30689500\end{array}$

C $\quad 0.36305300 \quad-1.11295600 \quad-0.48527000$

C $\quad-1.04958800-1.39330300 \quad-0.73084300$

$\begin{array}{llll}\mathrm{H} & -1.34746700 & -2.32185500 & -0.23862700\end{array}$

$\mathrm{H} \quad \quad-1.20221200 \quad-1.54665000 \quad-1.81219100$

$\mathrm{N} \quad \quad-1.91380900-0.30590200 \quad-0.21756500$

C $\quad-1.77300300 \quad 0.96651100 \quad-0.98509600$

$\begin{array}{llll}\mathrm{H} & -0.69568900 & 1.13387600 & -1.05033400\end{array}$

$\mathrm{H} \quad-2.17142200 \quad 0.82808600 \quad-2.00126500$

C $\quad-2.41646400 \quad 2.17290700 \quad-0.34974400$

$\begin{array}{llll}\text { C } & -1.71783600 & 3.15810800 & 0.21680000\end{array}$

$\mathrm{H} \quad-3.50016000 \quad 2.23498400 \quad-0.41713000$

$\begin{array}{llll}\mathrm{H} & -2.21395600 & 4.03396200 & 0.62662800\end{array}$

$\begin{array}{llll}\mathrm{H} & -0.63315000 & 3.12655600 & 0.27586800\end{array}$

S $\quad-3.50250400 \quad-0.79237600 \quad 0.17183500$

$\begin{array}{llll}\mathrm{O} & -4.46817700 & -0.12782100 & -0.71417800\end{array}$ 


$$
\begin{array}{lrrr}
\mathrm{O} & -3.49885600 & -2.25610100 & 0.29698800 \\
\mathrm{C} & -3.69688800 & -0.10613500 & 1.82532300 \\
\mathrm{H} & -4.72137300 & -0.31721500 & 2.13743300 \\
\mathrm{H} & -2.97981300 & -0.59815900 & 2.48192100 \\
\mathrm{H} & -3.51304300 & 0.96717200 & 1.78550400
\end{array}
$$

Electronic Energy $(\mathrm{EE})=-1220.990018$ a.u.

$\mathrm{EE}+$ Thermal Enthalpy Correction $=-1220.704679$ a.u.

$\mathrm{EE}+$ Thermal Free Energy Correction $=-1220.774094$ a.u.

Imaginary Freq $=0$

\section{${ }^{3} \mathrm{O}_{2}$}

$\begin{array}{llll}\text { O } & 0.00000000 & 0.00000000 & 0.60729600\end{array}$

$\begin{array}{lllll}\mathrm{O} & 0.00000000 & 0.00000000 & -0.60729600\end{array}$

Electronic Energy $(E E)=-150.320042$ a.u.

$\mathrm{EE}+$ Thermal Enthalpy Correction $=-150.312956$ a.u.

Imaginary Freq $=0$
${ }^{1} \mathbf{O}_{2}$
$\begin{array}{llll}\mathrm{O} & 0.00000000 & 0.00000000 & 0.60796400\end{array}$
$\begin{array}{lllll}\mathrm{O} & 0.00000000 & 0.00000000 & -0.60796400\end{array}$

Electronic Energy $(\mathrm{EE})=-150.257427$ a.u.

$\mathrm{EE}+$ Thermal Enthalpy Correction $=-150.250375$ a.u.

Imaginary Freq $=0$

\section{TS1}
C
$-2.87913900 \quad 1.68734400 \quad 0.36686500$
C
$\begin{array}{lll}-4.25540800 & 1.89909500 & 0.37174300\end{array}$
C
$\begin{array}{lll}-5.13385100 & 0.81611900 & 0.26395900\end{array}$
C
$\begin{array}{lll}-4.62898200 & -0.47507200 & 0.16066100\end{array}$
C
$\begin{array}{lll}-3.24718100 & -0.70383700 & 0.16316100\end{array}$
C
$\begin{array}{lll}-2.35829600 & 0.38762600 & 0.26636400\end{array}$
$\mathrm{H}$
$\begin{array}{lll}-2.19152200 & 2.52342000 & 0.44001900\end{array}$ 


\begin{tabular}{|c|c|c|c|}
\hline $\mathrm{H}$ & -4.64315900 & 2.90982200 & 0.45610800 \\
\hline $\mathrm{H}$ & -6.20664000 & 0.98323200 & 0.26358200 \\
\hline $\mathrm{H}$ & -5.28375400 & -1.33674300 & 0.08221800 \\
\hline $\mathrm{C}$ & -2.75628500 & -2.10534200 & 0.09524900 \\
\hline $\mathrm{O}$ & -3.48768900 & -3.06900400 & -0.02462800 \\
\hline $\mathrm{H}$ & -1.65930900 & -2.23058700 & 0.18253000 \\
\hline $\mathrm{C}$ & -0.90205300 & 0.22221100 & 0.25131500 \\
\hline $\mathrm{C}$ & 0.04388600 & 0.45775000 & 1.08324500 \\
\hline $\mathrm{C}$ & 1.45149400 & 0.50691900 & 1.50718900 \\
\hline $\mathrm{H}$ & 1.65169300 & -0.31010800 & 2.20684500 \\
\hline $\mathrm{H}$ & 1.64385200 & 1.44454800 & 2.04500200 \\
\hline $\mathrm{N}$ & 2.39634700 & 0.41022700 & 0.38153300 \\
\hline $\mathrm{C}$ & 2.90963000 & 1.67508500 & -0.18010800 \\
\hline $\mathrm{H}$ & 3.39327000 & 2.27026300 & 0.60786200 \\
\hline $\mathrm{H}$ & 3.68907400 & 1.41534900 & -0.90156000 \\
\hline $\mathrm{C}$ & 1.82590300 & 2.47793400 & -0.84673400 \\
\hline $\mathrm{C}$ & 1.55669600 & 3.74912600 & -0.55076200 \\
\hline $\mathrm{H}$ & 1.26116600 & 1.96009700 & -1.61986200 \\
\hline $\mathrm{H}$ & 0.79162800 & 4.30789800 & -1.08078400 \\
\hline $\mathrm{H}$ & 2.10243500 & 4.28336800 & 0.22361200 \\
\hline $\mathrm{O}$ & -1.24742000 & -0.76802500 & -2.01115400 \\
\hline $\mathrm{O}$ & -0.34946600 & -0.25006000 & -1.25591300 \\
\hline $\mathrm{S}$ & 3.34966100 & -0.98892600 & 0.24553900 \\
\hline $\mathrm{O}$ & 4.71443300 & -0.57440400 & -0.09224100 \\
\hline $\mathrm{O}$ & 3.07390000 & -1.81633900 & 1.42469200 \\
\hline $\mathrm{C}$ & 2.67273100 & -1.83601300 & -1.19183300 \\
\hline $\mathrm{H}$ & 3.24539000 & -2.75710200 & -1.31383700 \\
\hline $\mathrm{H}$ & 1.61963900 & -2.04924600 & -1.01413400 \\
\hline $\mathrm{H}$ & 2.79229800 & -1.19399800 & -2.06486200 \\
\hline
\end{tabular}

Electronic Energy $(\mathrm{EE})=-1371.258143$ a.u.

$\mathrm{EE}+$ Thermal Enthalpy Correction $=-1370.966873$ a.u. 


$$
\text { Imaginary Freq }=1
$$

\section{INT1}

C

$2.75188300 \quad 1.67143700 \quad-0.30096600$

$\mathrm{C}$

$\begin{array}{lll}4.11155100 & 1.97350200 & -0.37435700\end{array}$

C

$\begin{array}{lll}5.06078600 & 0.95069100 & -0.32048400\end{array}$

C

$\begin{array}{lll}4.64367800 & -0.37193500 & -0.20297200\end{array}$

C

$\begin{array}{lll}3.28227200 & -0.68905100 & -0.13712000\end{array}$

C

$\begin{array}{lll}2.32143600 & 0.34376400 & -0.17924900\end{array}$

$\mathrm{H}$

$\begin{array}{llll}2.01021700 & 2.46301300 & -0.32889000\end{array}$

$\mathrm{H}$

$\begin{array}{llll}4.42759500 & 3.00796000 & -0.46933600\end{array}$

$\mathrm{H}$

$\begin{array}{lll}6.11952600 & 1.18529700 & -0.37293300\end{array}$

$\mathrm{H}$

$\begin{array}{llll}5.35536400 & -1.19020600 & -0.16702100\end{array}$

C

$2.89048800-2.12146200-0.05954600$

$\mathrm{O}$

$\begin{array}{lll}3.69077800 & -3.03331800 & 0.01847500\end{array}$

$\mathrm{H}$

$\begin{array}{lll}1.80290000 & -2.32574500 & -0.09629100\end{array}$

$\mathrm{C}$

$\begin{array}{llll}0.85911700 & 0.09306000 & -0.12150600\end{array}$

C

$\begin{array}{lll}0.00038500 & 0.44266500 & -1.05828300\end{array}$

C

$\begin{array}{lll}-1.40842000 & 0.48399200 & -1.49769700\end{array}$

$\mathrm{H}$

$\begin{array}{lll}-1.60177500 & -0.35041900 & -2.17949800\end{array}$

$\mathrm{H}$

$\begin{array}{lll}-1.59166200 & 1.40692500 & -2.06445900\end{array}$

$\mathrm{N}$

$\begin{array}{lll}-2.36750200 & 0.41635900 & -0.38329000\end{array}$

C

$\begin{array}{lll}-2.84995400 & 1.69792300 & 0.16617000\end{array}$

$\mathrm{H} \quad-3.26993700 \quad 2.31887300 \quad-0.63810400$

$\mathrm{H} \quad \begin{array}{llll}\mathrm{H} & -3.67346600 & 1.46956300 & 0.84844900\end{array}$

$\begin{array}{llll}\text { C } & -1.76502000 & 2.44830100 & 0.89022500\end{array}$

$\begin{array}{llll}\text { C } & -1.42243700 & 3.70702600 & 0.61711400\end{array}$

$\mathrm{H} \quad \begin{array}{llll}-1.26591300 & 1.90351500 & 1.68983500\end{array}$

$\mathrm{H} \quad-0.66126800 \quad 4.22766500 \quad 1.18985800$

$\mathrm{H} \quad-1.90255600 \quad 4.26910400 \quad-0.18057700$

O $\quad \begin{array}{llll}1.18284800 & -0.86476300 & 1.98632700\end{array}$

O $\quad \begin{array}{llll}0.30185100 & -0.48711800 & 1.07481400\end{array}$ 


$$
\begin{array}{lrrr}
\mathrm{S} & -3.37545700 & -0.94825300 & -0.26521800 \\
\mathrm{O} & -4.74445800 & -0.48411800 & -0.02073500 \\
\mathrm{O} & -3.05746000 & -1.81445000 & -1.40520900 \\
\mathrm{C} & -2.81160400 & -1.77330800 & 1.23196200 \\
\mathrm{H} & -3.42007300 & -2.67261100 & 1.34251000 \\
\mathrm{H} & -1.75660000 & -2.02037700 & 1.12458300 \\
\mathrm{H} & -2.96596600 & -1.10452100 & 2.07925300
\end{array}
$$

Electronic Energy $(\mathrm{EE})=-1371.261674$ a.u.

$\mathrm{EE}+$ Thermal Enthalpy Correction $=-1370.968739$ a.u. Imaginary Freq $=0$

\section{TS2-a}

$\mathrm{C}$

$2.56453000-1.05168700 \quad-1.40692500$

$\begin{array}{llll}\text { C } & 3.74885500 & -1.78204300 & -1.52868600\end{array}$

C $\quad 4.84982600 \quad-1.46830200 \quad-0.73243400$

C $\quad 4.74778200 \quad-0.43262900 \quad 0.19351200$

$\begin{array}{llll}\text { C } & 3.56083400 & 0.29679700 & 0.33890200\end{array}$

$\begin{array}{llll}\text { C } & 2.44901500 & -0.00882900 & -0.48203800\end{array}$

$\mathrm{H} \quad \begin{array}{llll}1.71691500 & -1.27741500 & -2.04583700\end{array}$

$\mathrm{H} \quad 3.81236100 \quad-2.58574100 \quad-2.25612100$

$\mathrm{H} \quad 5.77622300 \quad-2.02527100 \quad-0.83106100$

$\mathrm{H} \quad \begin{array}{llll}5.59429400 & -0.18646700 & 0.83004500\end{array}$

$\begin{array}{llll}\text { C } & 3.52216900 & 1.31426900 & 1.42070700\end{array}$

$\begin{array}{lllll}\mathrm{O} & 2.54192400 & 1.93257900 & 1.78098000\end{array}$

$\mathrm{H} \quad 4.50300200 \quad 1.45963000 \quad 1.92627600$

$\begin{array}{llll}\text { C } & 1.17783300 & 0.75749300 & -0.42606300\end{array}$

$\begin{array}{lllll}\text { C } & -0.01730400 & 0.27655900 & -0.13480800\end{array}$

C $\quad-1.40434900 \quad 0.75615200 \quad-0.00395000$

$\mathrm{H} \quad-1.65445000 \quad 1.42245700 \quad-0.83665300$

$\begin{array}{llll}\mathrm{H} & -1.52049700 & 1.34540100 & 0.91833300\end{array}$

$\begin{array}{llll}\mathrm{N} & -2.30155700 & -0.42390900 & -0.01468400\end{array}$ 


$$
\begin{array}{lrrr}
\mathrm{C} & -1.88234000 & -1.44588800 & 0.96611400 \\
\mathrm{H} & -1.92809600 & -1.06914400 & 1.99885700 \\
\mathrm{H} & -2.57927300 & -2.28309500 & 0.88330500 \\
\mathrm{C} & -0.48099500 & -1.90052800 & 0.64932300 \\
\mathrm{C} & 0.43487800 & -2.17694200 & 1.60980700 \\
\mathrm{H} & -0.32421400 & -2.26978800 & -0.36179800 \\
\mathrm{H} & 1.38621200 & -2.63758400 & 1.36730400 \\
\mathrm{H} & 0.26904600 & -1.89903900 & 2.64675000 \\
\mathrm{O} & 0.36907000 & 2.91325500 & -0.75058200 \\
\mathrm{O} & 1.41178600 & 2.10367000 & -0.83437600 \\
\mathrm{~S} & -3.96603700 & -0.05444300 & -0.01216400 \\
\mathrm{O} & -4.66363500 & -1.27241900 & 0.41202400 \\
\mathrm{O} & -4.21176200 & 1.22367200 & 0.66687500 \\
\mathrm{C} & -4.27467600 & 0.18719100 & -1.77052700 \\
\mathrm{H} & -5.32740900 & 0.45900900 & -1.86471600 \\
\mathrm{H} & -3.64658900 & 0.99675400 & -2.14391500 \\
\mathrm{H} & -4.06545700 & -0.74698500 & -2.29031800
\end{array}
$$

Electronic Energy $(\mathrm{EE})=-1371.256899$ a.u.

$\mathrm{EE}+$ Thermal Enthalpy Correction $=-1370.965783$ a.u. Imaginary Freq $=1$

\section{TS2-b}
$\mathrm{C}$
$3.51631900-1.62746400 \quad 0.64633600$
C
$\begin{array}{lll}4.86952900 & -1.28800900 & 0.61314200\end{array}$
C
$\begin{array}{lll}5.28590700 & -0.06624700 & 0.07567300\end{array}$
C
$\begin{array}{lll}4.34829600 & 0.84365600 & -0.41908800\end{array}$
C
$\begin{array}{llll}2.99329800 & 0.52741600 & -0.36104400\end{array}$
C
$\begin{array}{lll}2.58028200 & -0.71610400 & 0.14941500\end{array}$
$\mathrm{H}$
$\begin{array}{lll}3.19564200 & -2.58830700 & 1.03371300\end{array}$
$\mathrm{H}$
$\begin{array}{lll}5.60575800 & -1.98826200 & 0.99564100\end{array}$
$\mathrm{H}$
$\begin{array}{lll}6.34365500 & 0.17581300 & 0.04033700\end{array}$ 


\begin{tabular}{|c|c|c|c|}
\hline $\mathrm{H}$ & 4.66153400 & 1.79470500 & -0.83834200 \\
\hline $\mathrm{C}$ & 1.89710400 & 1.45687400 & -0.79220300 \\
\hline $\mathrm{O}$ & 1.83312000 & 1.99782500 & -1.90702100 \\
\hline $\mathrm{H}$ & 1.30703000 & 1.87094800 & 0.05495100 \\
\hline $\mathrm{C}$ & 1.13949800 & -0.94848900 & 0.00326100 \\
\hline $\mathrm{C}$ & 0.38295800 & -0.03922700 & -0.61162400 \\
\hline $\mathrm{C}$ & -0.95857100 & 0.29041900 & -1.13228000 \\
\hline $\mathrm{H}$ & -1.27928400 & -0.51086800 & -1.80574800 \\
\hline $\mathrm{H}$ & -0.87946200 & 1.20814600 & -1.73296600 \\
\hline $\mathrm{N}$ & -1.99108900 & 0.45331900 & -0.09120900 \\
\hline $\mathrm{C}$ & -2.08610300 & 1.77737200 & 0.55296400 \\
\hline $\mathrm{H}$ & -1.99059200 & 2.57219800 & -0.20014800 \\
\hline $\mathrm{H}$ & -3.09492900 & 1.86526800 & 0.96697300 \\
\hline $\mathrm{C}$ & -1.07252400 & 1.96927400 & 1.64985800 \\
\hline $\mathrm{C}$ & -0.33746500 & 3.07104200 & 1.80676300 \\
\hline $\mathrm{H}$ & -0.99590200 & 1.15314600 & 2.36729100 \\
\hline $\mathrm{H}$ & 0.33799800 & 3.19086800 & 2.64806300 \\
\hline $\mathrm{H}$ & -0.39576200 & 3.89975300 & 1.10489800 \\
\hline $\mathrm{O}$ & -0.55213000 & -2.49015200 & 0.21391100 \\
\hline $\mathrm{O}$ & 0.69080600 & -2.15914000 & 0.53932000 \\
\hline$S$ & -3.45550700 & -0.40460900 & -0.31471300 \\
\hline $\mathrm{O}$ & -4.57215700 & 0.53717000 & -0.17080800 \\
\hline $\mathrm{O}$ & -3.30941800 & -1.21701100 & -1.52574400 \\
\hline $\mathrm{C}$ & -3.51042200 & -1.50842700 & 1.10667200 \\
\hline $\mathrm{H}$ & -4.43306900 & -2.08487300 & 1.01774300 \\
\hline $\mathrm{H}$ & -2.63600200 & -2.15694900 & 1.08336500 \\
\hline $\mathrm{H}$ & -3.53127200 & -0.90312700 & 2.01377700 \\
\hline
\end{tabular}

Electronic Energy $(\mathrm{EE})=-1371.251762$ a.u.

$\mathrm{EE}+$ Thermal Enthalpy Correction $=-1370.960701$ a.u. Imaginary Freq = 1 


\section{TS2-c}

\begin{tabular}{|c|c|c|c|}
\hline $\mathrm{C}$ & 2.44362200 & 1.79604200 & -0.05428600 \\
\hline $\mathrm{C}$ & 3.72729400 & 2.28746000 & 0.16530700 \\
\hline $\mathrm{C}$ & 4.81115900 & 1.40981700 & 0.26856400 \\
\hline $\mathrm{C}$ & 4.61407300 & 0.03428400 & 0.14469400 \\
\hline $\mathrm{C}$ & 3.33599100 & -0.46065300 & -0.09818800 \\
\hline $\mathrm{C}$ & 2.23937200 & 0.41373900 & -0.18783500 \\
\hline $\mathrm{H}$ & 1.59255800 & 2.46773800 & -0.10455300 \\
\hline $\mathrm{H}$ & 3.88280900 & 3.35713200 & 0.26698000 \\
\hline $\mathrm{H}$ & 5.80974400 & 1.79931300 & 0.44178300 \\
\hline $\mathrm{H}$ & 5.43657200 & -0.66816500 & 0.22154700 \\
\hline $\mathrm{C}$ & 3.09424000 & -1.94793900 & -0.27496400 \\
\hline $\mathrm{O}$ & 3.96268100 & -2.75558000 & 0.15451500 \\
\hline $\mathrm{H}$ & 2.63611800 & -2.21810800 & -1.25317400 \\
\hline $\mathrm{C}$ & 0.90170500 & -0.13504300 & -0.42706900 \\
\hline $\mathrm{C}$ & -0.09721000 & 0.50480800 & -1.05284200 \\
\hline $\mathrm{C}$ & -1.51224800 & 0.29696800 & -1.41838200 \\
\hline $\mathrm{H}$ & -1.64034900 & -0.70449000 & -1.84874200 \\
\hline $\mathrm{H}$ & -1.80720700 & 1.00905700 & -2.20239500 \\
\hline $\mathrm{N}$ & -2.42146000 & 0.42810000 & -0.26728600 \\
\hline $\mathrm{C}$ & -2.84929700 & 1.79521900 & 0.07831300 \\
\hline $\mathrm{H}$ & -3.24307800 & 2.30496200 & -0.81334800 \\
\hline $\mathrm{H}$ & -3.67560700 & 1.70541400 & 0.78814200 \\
\hline $\mathrm{C}$ & -1.72913000 & 2.59902800 & 0.68166700 \\
\hline $\mathrm{C}$ & -1.33582600 & 3.79127800 & 0.23060700 \\
\hline $\mathrm{H}$ & -1.25561900 & 2.16309800 & 1.56017400 \\
\hline $\mathrm{H}$ & -0.55777100 & 4.36122100 & 0.72908700 \\
\hline $\mathrm{H}$ & -1.79087100 & 4.24870300 & -0.64472800 \\
\hline $\mathrm{O}$ & 1.66831500 & -2.12279600 & 0.58626900 \\
\hline $\mathrm{O}$ & 0.59612500 & -1.40999900 & 0.02153100 \\
\hline $\mathrm{S}$ & -3.46631500 & -0.87393500 & 0.06524500 \\
\hline $\mathrm{O}$ & -4.70199000 & -0.31044400 & $\begin{array}{l}0.61673100 \\
\text { S32 }\end{array}$ \\
\hline
\end{tabular}




$$
\begin{array}{llll}
\mathrm{O} & -3.48888400 & -1.75051900 & -1.11105200 \\
\mathrm{C} & -2.62038500 & -1.76011500 & 1.38454800 \\
\mathrm{H} & -3.22275500 & -2.64148000 & 1.61191800 \\
\mathrm{H} & -1.62813800 & -2.04690700 & 1.03536000 \\
\mathrm{H} & -2.55526900 & -1.10588100 & 2.25426600
\end{array}
$$

Electronic Energy $(\mathrm{EE})=-1371.230179$ a.u.

$\mathrm{EE}+$ Thermal Enthalpy Correction $=-1370.939898$ a.u. Imaginary Freq = 1

\section{INT2}

C

$2.74717700 \quad-1.08966600 \quad-1.36115400$

C $\quad 4.00764100-1.69104200-1.39816000$

C $\quad 5.00970500 \quad-1.27024600 \quad-0.52513500$

C $\quad 4.73733500 \quad-0.25315500 \quad 0.38738400$

$\begin{array}{llll}\text { C } & 3.47401400 & 0.34718100 & 0.44480700\end{array}$

C $\quad 2.45722500 \quad-0.07545200 \quad-0.44365800$

$\mathrm{H} \quad 1.97802500-1.40107400 \quad-2.06113900$

$\begin{array}{llll}\mathrm{H} & 4.20674300 & -2.47836100 & -2.11887000\end{array}$

$\mathrm{H} \quad 5.99328300 \quad-1.72820900 \quad-0.55629700$

$\mathrm{H} \quad \begin{array}{llll}\mathrm{H} & 5.50860500 & 0.08044400 & 1.07738700\end{array}$

C $\quad 3.25169300 \quad 1.36649400 \quad 1.50037000$

$\begin{array}{llll}\text { O } & 2.18298700 & 1.86902800 & 1.78486100\end{array}$

$\mathrm{H} \quad 4.17351700 \quad 1.63662900 \quad 2.06167600$

$\begin{array}{lllll}\text { C } & 1.12492500 & 0.58931400 & -0.50979600\end{array}$

C $\quad-0.06234400 \quad 0.04289200 \quad-0.19897500$

C $\quad-1.42856000 \quad 0.66219500 \quad-0.37985100$

$\mathrm{H} \quad-1.57591400 \quad 1.08053100 \quad-1.37874600$

$\begin{array}{llll}\mathrm{H} & -1.59600200 & 1.47577300 & 0.33831900\end{array}$

$\mathrm{N} \quad \quad-2.33601300 \quad-0.48679200-0.14232500$

C $\quad-1.70400600 \quad-1.33513400 \quad 0.88730400$

$\mathrm{H} \quad-1.78072400 \quad-0.89691600 \quad 1.89223800$

$\begin{array}{llll}\mathrm{H} & -2.15498900 & -2.32636300 & 0.90068000\end{array}$ 


$$
\begin{array}{lrrr}
\mathrm{C} & -0.21780100 & -1.34679200 & 0.43000100 \\
\mathrm{C} & 0.72981800 & -1.66435200 & 1.53653300 \\
\mathrm{H} & -0.13053400 & -2.10295700 & -0.36249900 \\
\mathrm{H} & 1.07073300 & -2.67953200 & 1.70262800 \\
\mathrm{H} & 0.96456500 & -0.90769300 & 2.27723200 \\
\mathrm{O} & 0.23231700 & 2.65016400 & -1.09665400 \\
\mathrm{O} & 1.30247300 & 1.86232100 & -1.06290800 \\
\mathrm{~S} & -3.97770800 & -0.12415600 & 0.10501900 \\
\mathrm{O} & -4.56126000 & -1.30054700 & 0.75650200 \\
\mathrm{O} & -4.13828600 & 1.21218900 & 0.69284400 \\
\mathrm{C} & -4.57703600 & -0.05024500 & -1.59155100 \\
\mathrm{H} & -5.63734000 & 0.20229400 & -1.53660400 \\
\mathrm{H} & -4.03615100 & 0.72807500 & -2.13144300 \\
\mathrm{H} & -4.43298300 & -1.02579200 & -2.05446800
\end{array}
$$

Electronic Energy $(\mathrm{EE})=-1371.301649$ a.u.

$\mathrm{EE}+$ Thermal Enthalpy Correction $=-1371.008977$ a.u. Imaginary Freq $=0$

\section{TS3-a}
$\mathrm{C}$
$3.12862200 \quad 1.41558800 \quad-1.05957600$
C
$\begin{array}{lll}4.33035700 & 1.02829200 & -1.65289800\end{array}$
C
$\begin{array}{lll}4.88092300 & -0.22017200 & -1.36388800\end{array}$
C
$\begin{array}{llll}4.23703200 & -1.06406700 & -0.45968100\end{array}$
C
$\begin{array}{lll}3.05047500 & -0.67278300 & 0.16792400\end{array}$
C
$\begin{array}{llll}2.47039200 & 0.56936500 & -0.15743000\end{array}$
$\mathrm{H}$
$\begin{array}{lll}2.68692100 & 2.37602200 & -1.30469800\end{array}$
$\mathrm{H}$
$\begin{array}{lll}4.82679700 & 1.69718200 & -2.34910300\end{array}$
$\mathrm{H}$
$\begin{array}{llll}5.81012600 & -0.53098300 & -1.83148900\end{array}$
$\mathrm{H}$
$\begin{array}{lll}4.67452500 & -2.02691200 & -0.20704400\end{array}$
C
$2.49926800-1.55078300 \quad 1.25781800$
$\mathrm{O}$
$2.02746100-1.08220400 \quad 2.31331800$
$\mathrm{H}$
$\begin{array}{lll}2.91829300 & -2.57793900 & 1.24878400\end{array}$ 


$\begin{array}{llll}\mathrm{C} & 1.14511400 & 0.97302400 & 0.37285800 \\ \mathrm{C} & 0.01537800 & 0.25182100 & 0.25119300 \\ \mathrm{C} & -1.39850800 & 0.67911300 & 0.53634700 \\ \mathrm{H} & -1.55735100 & 1.74598800 & 0.37138500 \\ \mathrm{H} & -1.69288800 & 0.46070800 & 1.57374300 \\ \mathrm{~N} & -2.15750700 & -0.13187400 & -0.44551000 \\ \mathrm{C} & -1.51469000 & -1.45947800 & -0.53433500 \\ \mathrm{H} & -1.83009300 & -2.12260700 & 0.28300300 \\ \mathrm{H} & -1.76631200 & -1.94034700 & -1.47922600 \\ \mathrm{C} & 0.00368200 & -1.13372500 & -0.40353000 \\ \mathrm{C} & 0.70512100 & -2.24111000 & 0.34352400 \\ \mathrm{H} & 0.44579200 & -1.04519800 & -1.40281700 \\ \mathrm{H} & 1.11725000 & -3.04929600 & -0.25751500 \\ \mathrm{H} & 0.25976200 & -2.55728000 & 1.28232800 \\ \mathrm{O} & 0.14004600 & 2.70873900 & 1.53444800 \\ \mathrm{O} & 1.20590600 & 2.27432700 & 0.86620800 \\ \mathrm{~S} & -3.84923200 & -0.16020100 & -0.27004200 \\ \mathrm{O} & -4.31513100 & -1.31893400 & -1.03744000 \\ \mathrm{O} & -4.23012200 & 0.00427500 & 1.13871400 \\ \mathrm{H} & -4.31594800 & 1.34132300 & -1.14629900 \\ \mathrm{H} & -5.40163800 & 1.42082900 & -1.06882900 \\ \mathrm{H} & -3.84407200 & 2.20009300 & -0.66713900 \\ \mathrm{H} & -4.00544700 & 1.24798900 & -2.18628200\end{array}$

Electronic Energy $(\mathrm{EE})=-1371.285989$ a.u.

$\mathrm{EE}+$ Thermal Enthalpy Correction $=-1370.99325$ a.u. Imaginary Freq = 1

\section{TS3-b}

C $2.63011700-1.63272500-0.66956800$

C $\quad 3.85830800 \quad-2.27710800 \quad-0.54488900$

C $\quad 4.97296300 \quad-1.58380900 \quad-0.06667600$ 


\begin{tabular}{|c|c|c|c|}
\hline $\mathrm{C}$ & 4.82833000 & -0.25206700 & 0.31081200 \\
\hline $\mathrm{C}$ & 3.59224300 & 0.40243200 & 0.22698600 \\
\hline $\mathrm{C}$ & 2.46160600 & -0.28367700 & -0.29994900 \\
\hline $\mathrm{H}$ & 1.78986300 & -2.16215800 & -1.10724000 \\
\hline $\mathrm{H}$ & 3.95173500 & -3.31515500 & -0.85023300 \\
\hline $\mathrm{H}$ & 5.93678500 & -2.07637700 & 0.01175200 \\
\hline $\mathrm{H}$ & 5.67973100 & 0.29496100 & 0.70904500 \\
\hline $\mathrm{C}$ & 3.51477100 & 1.74853300 & 0.83935300 \\
\hline $\mathrm{O}$ & 2.49285200 & 2.34808700 & 1.11000700 \\
\hline $\mathrm{H}$ & 4.50861200 & 2.17756300 & 1.09808100 \\
\hline $\mathrm{C}$ & 1.17258300 & 0.35708100 & -0.55681500 \\
\hline $\mathrm{C}$ & -0.08423100 & -0.10431200 & -0.22326400 \\
\hline $\mathrm{C}$ & -1.38073700 & 0.35144300 & -0.88437300 \\
\hline $\mathrm{H}$ & -1.25355300 & 0.34170800 & -1.97408400 \\
\hline $\mathrm{H}$ & -1.68407400 & 1.35375300 & -0.58125100 \\
\hline $\mathrm{N}$ & -2.39656600 & -0.65151000 & -0.47721100 \\
\hline $\mathrm{C}$ & -1.74005900 & -1.80509100 & 0.18236500 \\
\hline $\mathrm{H}$ & -2.37969500 & -2.20776300 & 0.96799800 \\
\hline $\mathrm{H}$ & -1.56309900 & -2.59212300 & -0.56059000 \\
\hline $\mathrm{C}$ & -0.40748800 & -1.25738400 & 0.69210600 \\
\hline $\mathrm{C}$ & -0.47795000 & -0.18309400 & 1.70556500 \\
\hline $\mathrm{H}$ & 0.36478200 & -2.00962300 & 0.84564000 \\
\hline $\mathrm{H}$ & -1.39650700 & 0.35998900 & 1.89472700 \\
\hline $\mathrm{H}$ & 0.41605800 & 0.10776200 & 2.24077700 \\
\hline $\mathrm{O}$ & 0.51579700 & 2.54849800 & -0.95147400 \\
\hline $\mathrm{O}$ & 1.29564100 & 1.53682900 & -1.34984500 \\
\hline S & -3.83529800 & -0.06847700 & 0.21261900 \\
\hline $\mathrm{O}$ & -4.46008700 & -1.20184500 & 0.90221000 \\
\hline $\mathrm{O}$ & -3.60435100 & 1.19717600 & 0.92489300 \\
\hline $\mathrm{C}$ & -4.80237000 & 0.31544200 & -1.25553500 \\
\hline $\mathrm{H}$ & -5.75108300 & 0.72915800 & -0.90935700 \\
\hline
\end{tabular}




$$
\begin{aligned}
& \mathrm{H} \quad-4.26629600 \quad 1.05499300 \quad-1.85196500 \\
& \mathrm{H} \quad-4.95985100 \quad-0.60443100 \quad-1.81774800
\end{aligned}
$$

Electronic Energy $(\mathrm{EE})=-1371.284108$ a.u.

$\mathrm{EE}+$ Thermal Enthalpy Correction $=-1370.992024$ a.u.

Imaginary Freq $=1$

\section{INT3}

C

$3.27754700 \quad 1.45324300 \quad-0.86950900$

C $\quad 4.52051300 \quad 1.07350800 \quad-1.36752000$

C $\quad 4.98629700 \quad-0.22644000 \quad-1.16518000$

$\begin{array}{llll}\text { C } & 4.20772900 & -1.13087700 & -0.44816400\end{array}$

C $\quad 2.94703000 \quad-0.77132400 \quad 0.04198300$

$\begin{array}{llll}\text { C } & 2.47144300 & 0.54402000 & -0.16424200\end{array}$

$\mathrm{H} \quad 2.91876500 \quad 2.46452200 \quad-1.02487200$

$\mathrm{H} \quad \begin{array}{llll}5.12426400 & 1.79293500 & -1.91198600\end{array}$

$\mathrm{H} \quad 5.95432300 \quad-0.53042500 \quad-1.55101600$

$\mathrm{H} \quad 4.57904600 \quad-2.13364000 \quad-0.25889600$

C $\quad 2.17948300 \quad-1.78403500 \quad 0.90315100$

O $\quad 2.12907100 \quad-1.14736900 \quad 2.13052300$

$\mathrm{H} \quad 2.79787600 \quad-2.69619700 \quad 0.95724200$

$\begin{array}{llll}\text { C } & 1.14383900 & 0.99377600 & 0.31047100\end{array}$

$\begin{array}{llll}\text { C } & 0.00199400 & 0.28727200 & 0.21797700\end{array}$

$\begin{array}{llll}\text { C } & -1.40703800 & 0.74971700 & 0.47457400\end{array}$

$\mathrm{H} \quad-1.56003700 \quad 1.80107500 \quad 0.22407300$

$\mathrm{H} \quad-1.69425900 \quad 0.62104100 \quad 1.52853200$

$\mathrm{N} \quad \quad-2.18055600 \quad-0.13498300 \quad-0.42996200$

C $\quad-1.53033000 \quad-1.46312900 \quad-0.43333500$

$\mathrm{H} \quad-1.81966700 \quad-2.06143700 \quad 0.44227500$

$\mathrm{H} \quad-1.81091000 \quad-2.01610900-1.32976000$

C $\quad-0.02525900-1.11872800 \quad-0.36470100$

$\begin{array}{llll}\text { C } & 0.76995900 & -2.18542400 & 0.40873900\end{array}$ 


$\begin{array}{lrrr}\mathrm{H} & 0.38143600 & -1.06458300 & -1.38343600 \\ \mathrm{H} & 0.86888600 & -3.07429800 & -0.22660400 \\ \mathrm{H} & 0.21104100 & -2.49859500 & 1.29841900 \\ \mathrm{O} & 0.14979800 & 2.79042900 & 1.38462500 \\ \mathrm{O} & 1.21873600 & 2.31529000 & 0.75138500 \\ \mathrm{~S} & -3.86713100 & -0.14537300 & -0.23127900 \\ \mathrm{O} & -4.34593700 & -1.36893400 & -0.88116400 \\ \mathrm{O} & -4.22918900 & 0.15177700 & 1.16094800 \\ \mathrm{C} & -4.34898400 & 1.26786100 & -1.23703000 \\ \mathrm{H} & -5.43340200 & 1.35549000 & -1.15118800 \\ \mathrm{H} & -3.87031800 & 2.16744600 & -0.84805500 \\ \mathrm{H} & -4.05421800 & 1.07744800 & -2.26835300\end{array}$

Electronic Energy $(\mathrm{EE})=-1371.306730$ a.u.

$\mathrm{EE}+$ Thermal Enthalpy Correction $=-1371.010742$ a.u.

Imaginary Freq $=0$

\section{TS4}

$\begin{array}{lrrr}\mathrm{C} & 3.59518400 & 1.27597600 & -0.83805400 \\ \mathrm{C} & 4.88921900 & 0.76948700 & -0.93352300 \\ \mathrm{C} & 5.18079100 & -0.50028000 & -0.43200300 \\ \mathrm{C} & 4.17797500 & -1.26133100 & 0.17629200 \\ \mathrm{C} & 2.88259000 & -0.76484100 & 0.28980900 \\ \mathrm{C} & 2.58136500 & 0.50712500 & -0.24397400 \\ \mathrm{H} & 3.36128600 & 2.26144100 & -1.22682600 \\ \mathrm{H} & 5.66961300 & 1.36662900 & -1.39469100 \\ \mathrm{H} & 6.19190900 & -0.89117000 & -0.49481400 \\ \mathrm{H} & 4.41644100 & -2.23663500 & 0.59310000 \\ \mathrm{C} & 1.74910500 & -1.54339400 & 0.95446200 \\ \mathrm{O} & 0.90789000 & -0.72664500 & 1.70780500 \\ \mathrm{H} & 2.18483500 & -2.28032900 & 1.64899500 \\ \mathrm{C} & 1.21570400 & 0.99358900 & -0.09621000\end{array}$




$\begin{array}{llll}\mathrm{C} & 0.08628600 & 0.21508800 & -0.03831500 \\ \mathrm{C} & -1.28258700 & 0.75993300 & 0.29153900 \\ \mathrm{H} & -1.41869700 & 1.79620600 & -0.02870100 \\ \mathrm{H} & -1.44665800 & 0.71277200 & 1.37518400 \\ \mathrm{~N} & -2.18028000 & -0.15096600 & -0.45471900 \\ \mathrm{C} & -1.55421100 & -1.48900000 & -0.42656200 \\ \mathrm{H} & -1.66349700 & -1.97129600 & 0.55425600 \\ \mathrm{H} & -1.99636900 & -2.13276700 & -1.18683500 \\ \mathrm{C} & -0.06983600 & -1.16937100 & -0.67938900 \\ \mathrm{C} & 0.89570600 & -2.24657300 & -0.12283700 \\ \mathrm{H} & 0.08547900 & -1.04241800 & -1.75732900 \\ \mathrm{H} & 1.51208000 & -2.68709000 & -0.91164700 \\ \mathrm{H} & 0.32005200 & -3.05650700 & 0.33774400 \\ \mathrm{O} & 0.56254500 & 2.77886000 & 1.21248500 \\ \mathrm{O} & 1.12013900 & 2.40070900 & 0.05637600 \\ \mathrm{~S} & -3.83339500 & -0.09078400 & -0.06115500 \\ \mathrm{O} & -4.40644200 & -1.36199400 & -0.51235100 \\ \mathrm{O} & -4.02185400 & 0.37055900 & 1.31987000 \\ \mathrm{C} & -4.40482500 & 1.21770100 & -1.15819400 \\ \mathrm{H} & -5.46954400 & 1.35000600 & -0.95838600 \\ \mathrm{H} & -3.86576400 & 2.13868200 & -0.93187300 \\ \mathrm{H} & -4.23789400 & 0.90522000 & -2.18838700 \\ \mathrm{H} & & & \end{array}$

Electronic Energy $(E E)=-1371.300581$ a.u.

$\mathrm{EE}+$ Thermal Enthalpy Correction $=-1371.005664$ a.u.

Imaginary Freq = 1
${ }^{3}$ INT4
C
$3.72519800 \quad 1.34258900 \quad-0.30329500$
C
$\begin{array}{llll}5.01075000 & 0.82620500 & -0.22760500\end{array}$
C
$\begin{array}{lll}5.21953300 & -0.51878700 & 0.10449400\end{array}$
$\mathrm{C}$
$\begin{array}{lll}4.12484300 & -1.35536800 & 0.36321200\end{array}$ 


\begin{tabular}{|c|c|c|c|}
\hline $\mathrm{C}$ & 2.82971800 & -0.86658300 & 0.29034200 \\
\hline $\mathrm{C}$ & 2.60956400 & 0.50647700 & -0.04739600 \\
\hline $\mathrm{H}$ & 3.56123900 & 2.38444100 & -0.55554000 \\
\hline $\mathrm{H}$ & 5.86084100 & 1.47261400 & -0.42347200 \\
\hline $\mathrm{H}$ & 6.22876700 & -0.91292000 & 0.16976800 \\
\hline $\mathrm{H}$ & 4.29079500 & -2.39630000 & 0.62965000 \\
\hline $\mathrm{C}$ & 1.59860000 & -1.71520900 & 0.53741800 \\
\hline $\mathrm{O}$ & 0.61380900 & -0.91362500 & 1.20841300 \\
\hline $\mathrm{H}$ & 1.83044100 & -2.56235800 & 1.18704200 \\
\hline $\mathrm{C}$ & 1.26446900 & 0.93513700 & -0.12298700 \\
\hline $\mathrm{C}$ & 0.14328500 & -0.02355700 & 0.18281500 \\
\hline $\mathrm{C}$ & -1.20951600 & 0.57077800 & 0.59853100 \\
\hline $\mathrm{H}$ & -1.24835700 & 1.65535900 & 0.48184200 \\
\hline $\mathrm{H}$ & -1.39655000 & 0.31964300 & 1.64728400 \\
\hline $\mathrm{N}$ & -2.17649800 & -0.07908000 & -0.32013700 \\
\hline $\mathrm{C}$ & -1.61714300 & -1.37159300 & -0.77377100 \\
\hline $\mathrm{H}$ & -1.69949900 & -2.15216900 & -0.00538500 \\
\hline $\mathrm{H}$ & -2.13543000 & -1.70919400 & -1.67140600 \\
\hline $\mathrm{C}$ & -0.14542600 & -1.00655200 & -1.00440200 \\
\hline $\mathrm{C}$ & 0.88253600 & -2.13994500 & -0.78297700 \\
\hline $\mathrm{H}$ & -0.03098200 & -0.50616700 & -1.96918900 \\
\hline $\mathrm{H}$ & 1.58416900 & -2.24282900 & -1.61354000 \\
\hline $\mathrm{H}$ & 0.38162800 & -3.10266700 & -0.64417200 \\
\hline $\mathrm{O}$ & 0.80013000 & 3.11607400 & 0.33302900 \\
\hline $\mathrm{O}$ & 0.96553300 & 2.18970500 & -0.64585900 \\
\hline$S$ & -3.80891700 & -0.07237600 & 0.13883700 \\
\hline $\mathrm{O}$ & -4.45215000 & -1.14968400 & -0.61929000 \\
\hline $\mathrm{O}$ & -3.93551000 & -0.00340700 & 1.60028200 \\
\hline $\mathrm{C}$ & -4.36179300 & 1.50645900 & -0.52795700 \\
\hline $\mathrm{H}$ & -5.41232900 & 1.61324200 & -0.25211000 \\
\hline $\mathrm{H}$ & -3.77493000 & 2.31040500 & -0.08147700 \\
\hline
\end{tabular}


Electronic Energy $(\mathrm{EE})=-1371.347186$ a.u.

$\mathrm{EE}+$ Thermal Enthalpy Correction $=-1371.050192$ a.u.

Imaginary Freq $=0$

\section{${ }^{1}$ INT4}

$\mathrm{C}$

$\begin{array}{lll}3.71251900 & 1.50428000 & 0.10649000\end{array}$

C

$\begin{array}{lll}5.02402300 & 1.04416100 & 0.11590300\end{array}$

$\mathrm{C}$

$\begin{array}{lll}5.28689500 & -0.32948500 & 0.15888300\end{array}$

C

$\begin{array}{lll}4.23582700 & -1.24812700 & 0.19732900\end{array}$

C

$2.91586300 \quad-0.80491900 \quad 0.19640700$

C

$\begin{array}{lll}2.65065900 & 0.58409700 & 0.14810100\end{array}$

$\mathrm{H}$

$\begin{array}{lll}3.49724500 & 2.56640200 & 0.06587200\end{array}$

$\mathrm{H}$

$\begin{array}{lll}5.84495300 & 1.75364900 & 0.09144200\end{array}$

$\mathrm{H}$

$\begin{array}{lll}6.31305000 & -0.68401500 & 0.17127000\end{array}$

$\mathrm{H}$

$\begin{array}{lll}4.44488000 & -2.31365900 & 0.23906900\end{array}$

C

$\begin{array}{lll}1.71865600 & -1.73012100 & 0.25481100\end{array}$

$\mathrm{O}$

$\begin{array}{lll}0.74118400 & -1.10050400 & 1.09247900\end{array}$

$\mathrm{H}$

$\begin{array}{lll}1.98606300 & -2.68453700 & 0.71408400\end{array}$

C

$\begin{array}{lll}1.25307300 & 0.97909900 & 0.04901900\end{array}$

C

$\begin{array}{lll}0.18578100 & -0.06625100 & 0.27418000\end{array}$

C

$\begin{array}{lll}-1.16773700 & 0.35219500 & 0.87368200\end{array}$

$\mathrm{H}$

$\begin{array}{lll}-1.25687300 & 1.43039000 & 0.99124500\end{array}$

$\mathrm{H}$

$\begin{array}{lll}-1.28185100 & -0.15447600 & 1.83772100\end{array}$

$\mathrm{N}$

$\begin{array}{lll}-2.12804300 & -0.13577500 & -0.13418500\end{array}$

C $\quad-1.55728800 \quad-1.26835200 \quad-0.87686800$

$\mathrm{H} \quad-1.58175000 \quad-2.20414400 \quad-0.30059400$

$\mathrm{H} \quad-2.09863000 \quad-1.42235400 \quad-1.81061400$

C $\quad-0.10504900-0.80234300 \quad-1.07883100$

C $\quad 0.98124700 \quad-1.90167500 \quad-1.11320200$

$\mathrm{H} \quad-0.05564500 \quad-0.10551200-1.91805400$ 


$$
\begin{array}{lrrr}
\mathrm{H} & 1.66258100 & -1.79570800 & -1.96039700 \\
\mathrm{H} & 0.53123200 & -2.89688800 & -1.16754200 \\
\mathrm{O} & -0.27939800 & 2.50454800 & -0.71119500 \\
\mathrm{O} & 1.01350900 & 2.15065200 & -0.40815900 \\
\mathrm{~S} & -3.76986700 & -0.10459800 & 0.22493600 \\
\mathrm{O} & -4.40318300 & -1.11341200 & -0.63092500 \\
\mathrm{O} & -3.96057000 & -0.11900600 & 1.68040900 \\
\mathrm{C} & -4.24500900 & 1.52862600 & -0.36689600 \\
\mathrm{H} & -5.29515800 & 1.67232000 & -0.10670200 \\
\mathrm{H} & -3.62371900 & 2.27804500 & 0.12510000 \\
\mathrm{H} & -4.10129000 & 1.55915800 & -1.44650400
\end{array}
$$

Electronic Energy $(\mathrm{EE})=-1371.383398$ a.u.

$\mathrm{EE}+$ Thermal Enthalpy Correction $=-1371.084311$ a.u. Imaginary Freq $=0$

\section{TS5}
$\mathrm{C}$
$2.19412800 \quad-3.53521800 \quad 0.71450300$
C
$2.88097500-4.61053700 \quad 1.26647700$
C
$\begin{array}{lll}4.17719900 & -4.44084500 & 1.76441100\end{array}$
C
$\begin{array}{lll}4.79104100 & -3.18696900 & 1.72681200\end{array}$
C
$\begin{array}{lll}4.11729700 & -2.09303900 & 1.19068400\end{array}$
C
$2.81367900-2.27162900 \quad 0.66938700$
$\mathrm{H}$
$\begin{array}{lll}1.18644900 & -3.66065400 & 0.33045200\end{array}$
$\mathrm{H}$
$\begin{array}{lll}2.40312200 & -5.58382200 & 1.31096600\end{array}$
$\mathrm{H}$
$\begin{array}{lll}4.70606700 & -5.28505700 & 2.19706400\end{array}$
$\mathrm{H}$
$\begin{array}{lll}5.79073800 & -3.05681300 & 2.13265200\end{array}$
C
$\begin{array}{lll}4.69585000 & -0.69542000 & 1.15758500\end{array}$
$\mathrm{C}$
$2.18681400-1.13051900 \quad 0.01014200$
C
$\begin{array}{lll}2.90065900 & 0.20234700 & 0.09726300\end{array}$
C
$\begin{array}{lll}2.20683700 & 1.55855900 & -0.04713800\end{array}$
$\mathrm{H}$
$\begin{array}{lll}1.84340500 & 1.89932400 & 0.92276700\end{array}$ 


\begin{tabular}{|c|c|c|c|}
\hline $\mathrm{H}$ & 1.37427700 & 1.52016200 & -0.74526400 \\
\hline $\mathrm{N}$ & 3.35340100 & 2.38138600 & -0.51510400 \\
\hline $\mathrm{C}$ & 4.03515900 & 1.61315300 & -1.57645300 \\
\hline $\mathrm{H}$ & 3.47872000 & 1.63942400 & -2.52283400 \\
\hline $\mathrm{H}$ & 5.03482800 & 2.01345100 & -1.74966900 \\
\hline $\mathrm{C}$ & 4.03616000 & 0.17577900 & -1.01681000 \\
\hline $\mathrm{C}$ & 5.28107300 & -0.26549200 & -0.21334600 \\
\hline $\mathrm{H}$ & 5.94836600 & 0.58567400 & -0.05228300 \\
\hline $\mathrm{H}$ & 5.84666700 & -1.06060500 & -0.70498300 \\
\hline $\mathrm{O}$ & 0.60086300 & -0.39837400 & -1.48490800 \\
\hline $\mathrm{O}$ & 1.21514000 & -1.41841200 & -0.76250100 \\
\hline S & 3.07366900 & 4.01727900 & -0.83225800 \\
\hline $\mathrm{O}$ & 4.20738100 & 4.47111900 & -1.64634300 \\
\hline $\mathrm{O}$ & 1.69631800 & 4.25604300 & -1.28097500 \\
\hline $\mathrm{C}$ & 3.23601900 & 4.71824300 & 0.81946900 \\
\hline $\mathrm{H}$ & 3.04269400 & 5.78812900 & 0.72514800 \\
\hline $\mathrm{H}$ & 2.49692000 & 4.26048700 & 1.47796700 \\
\hline $\mathrm{H}$ & 4.24799400 & 4.53390100 & 1.17789700 \\
\hline $\mathrm{O}$ & -0.46586500 & 0.40870100 & -0.00237600 \\
\hline $\mathrm{H}$ & 3.79983200 & -0.53129500 & -1.81487000 \\
\hline $\mathrm{O}$ & 3.61364800 & 0.21791400 & 1.33681200 \\
\hline $\mathrm{H}$ & 5.40098200 & -0.54882100 & 1.97985300 \\
\hline $\mathrm{C}$ & -3.25107400 & 3.36132200 & 1.71015200 \\
\hline $\mathrm{C}$ & -4.16994100 & 4.40389500 & 1.74925000 \\
\hline $\mathrm{C}$ & -5.43640800 & 4.23847200 & 1.17869900 \\
\hline $\mathrm{C}$ & -5.78883500 & 3.02889600 & 0.57714900 \\
\hline $\mathrm{C}$ & -4.88637000 & 1.96687800 & 0.54893700 \\
\hline $\mathrm{C}$ & -3.60462300 & 2.13824500 & 1.12116100 \\
\hline $\mathrm{H}$ & -2.25236000 & 3.47833000 & 2.11667900 \\
\hline $\mathrm{H}$ & -3.90119300 & 5.34799100 & 2.21245900 \\
\hline $\mathrm{H}$ & -6.15311600 & 5.05395300 & 1.20698500 \\
\hline
\end{tabular}




$$
\begin{aligned}
& \begin{array}{llll}
\mathrm{H} & -6.77709300 & 2.90634500 & 0.14172200
\end{array} \\
& \begin{array}{llll}
\text { C } & -5.21749000 & 0.61098600 & -0.04578300
\end{array} \\
& \begin{array}{llll}
\text { C } & -2.61506400 & 1.05998900 & 0.93359100
\end{array} \\
& \text { C } \quad-3.15483600 \quad-0.22390100 \quad 0.33633700 \\
& \text { C } \quad-2.45678100 \quad-1.54946200 \quad 0.61542900 \\
& \mathrm{H} \quad-2.90461900 \quad-2.03426000 \quad 1.48677500 \\
& \begin{array}{llll}
\mathrm{H} & -1.38564500 & -1.39885300 & 0.74395200
\end{array} \\
& \begin{array}{llll}
\mathrm{N} & -2.78523600 & -2.29513400 & -0.62307300
\end{array} \\
& \text { C } \quad-2.66236600 \quad-1.37159500 \quad-1.78379600 \\
& \mathrm{H} \quad-1.61848700 \quad-1.25102700 \quad-2.08772400 \\
& \mathrm{H} \quad-3.25174800 \quad-1.75265000 \quad-2.61956600 \\
& \text { C } \quad-3.18683800-0.03082000-1.22644000 \\
& \begin{array}{llll}
\text { C } & -4.66432000 & 0.35463700 & -1.47589300
\end{array} \\
& \mathrm{H} \quad \begin{array}{llll}
\mathrm{H} & -5.21658100 & -0.48488800 & -1.90860000
\end{array} \\
& \mathrm{H} \quad-4.77360600 \quad 1.21704400 \quad-2.13824500 \\
& \begin{array}{llll}
\text { O } & -1.39940600 & 1.29956800 & 1.08080400
\end{array} \\
& \text { S } \quad-2.19864300 \quad-3.85791700 \quad-0.77976700 \\
& \begin{array}{llll}
\text { O } & -2.13488100 & -4.16079700 & -2.21257100
\end{array} \\
& \text { O } \quad-1.02166200 \quad-4.07337200 \quad 0.08164700 \\
& \begin{array}{llll}
\text { C } & -3.53867900 & -4.82310300 & -0.05697400
\end{array} \\
& \mathrm{H} \quad-3.22824900 \quad-5.86918300 \quad-0.07750000 \\
& \mathrm{H} \quad-3.69884300 \quad-4.49678600 \quad 0.97133800 \\
& \mathrm{H} \quad-4.43519300 \quad-4.67092000 \quad-0.65713500 \\
& \mathrm{H} \quad-2.49677200 \quad 0.75946900-1.52373000 \\
& \text { O } \quad-4.52538400 \quad-0.36487900 \quad 0.73137300 \\
& \mathrm{H} \quad-6.28991800 \quad 0.41118500 \quad 0.03082800
\end{aligned}
$$

Electronic Energy $(\mathrm{EE})=-2742.754907$ a.u.

$\mathrm{EE}+$ Thermal Enthalpy Correction $=-2742.156246$ a.u. Imaginary Freq = 1 
$\begin{array}{llll}-3.90039600 & 1.09791500 & -0.08908300\end{array}$

C

$\begin{array}{llll}-5.00910400 & 0.25716200 & -0.10227900\end{array}$

C

$\begin{array}{lll}-4.82844500 & -1.12798700 & -0.17053800\end{array}$

C

$\begin{array}{lll}-3.54426000 & -1.67341900 & -0.22869700\end{array}$

$\mathrm{C}$

$\begin{array}{llll}-2.42525300 & -0.84028200 & -0.21926900\end{array}$

C

$\begin{array}{lll}-2.61064000 & 0.55654000 & -0.14366900\end{array}$

$\mathrm{H}$

$\begin{array}{lll}-4.00470800 & 2.17653000 & -0.02913100\end{array}$

$\mathrm{H}$

$\begin{array}{lll}-6.01089800 & 0.67355600 & -0.06102500\end{array}$

$\mathrm{H}$

$\begin{array}{lll}-5.69253000 & -1.78592200 & -0.18910700\end{array}$

$\mathrm{H}$

$-3.41500800-2.75045300-0.29888900$

C

$\begin{array}{lll}-1.00514100 & -1.37106600 & -0.30077200\end{array}$

$\mathrm{O}$

$\begin{array}{lll}-0.19612600 & -0.40097700 & -0.97278800\end{array}$

$\mathrm{H}$

$\begin{array}{lll}-0.96426800 & -2.29911000 & -0.87644900\end{array}$

$\mathrm{C}$

$\begin{array}{lll}-1.41625200 & 1.44722700 & -0.06688700\end{array}$

C $\quad-0.09638400 \quad 0.67767600 \quad-0.03458900$

C $\quad 1.17724400 \quad 1.47298200 \quad-0.28973900$

$\mathrm{H} \quad \begin{array}{llll}1.06249500 & 2.48773500 & 0.10336500\end{array}$

$\mathrm{H} \quad \begin{array}{llll}1.43369200 & 1.52045900 & -1.34725000\end{array}$

$\mathrm{N} \quad 2.23065100 \quad 0.77396300 \quad 0.47873100$

$\begin{array}{llll}\text { C } & 1.58993300 & 0.25133900 & 1.70763200\end{array}$

$\mathrm{H} \quad 2.11122200 \quad-0.63825700 \quad 2.06279100$

$\mathrm{H} \quad \begin{array}{llll}1.66386000 & 1.02770900 & 2.47711800\end{array}$

C $\quad 0.10216700 \quad-0.01945900 \quad 1.36292700$

C $\quad-\quad \begin{array}{llll}0.30487300 & -1.48419500 & 1.07618500\end{array}$

$\mathrm{H} \quad-0.54180700 \quad 0.42569700 \quad 2.12626600$

$\mathrm{H} \quad-0.94585800 \quad-1.90927900 \quad 1.85259400$

$\mathrm{H} \quad 0.58186100 \quad-2.11224700 \quad 0.95916400$

S $\quad 3.24284800 \quad-0.30038700 \quad-0.38045100$

O $\quad 3.03037500 \quad-1.68252500 \quad 0.07739000$

O $\quad 3.16562800 \quad 0.04634100 \quad-1.80151100$ 


$$
\begin{array}{llll}
\mathrm{C} & 4.86452300 & 0.20379900 & 0.22164000 \\
\mathrm{H} & 4.89626100 & 0.08065700 & 1.30477600 \\
\mathrm{H} & 5.59827400 & -0.44812400 & -0.25563900 \\
\mathrm{H} & 5.02958700 & 1.24396600 & -0.05773000 \\
\mathrm{O} & -1.48250500 & 2.66292800 & 0.02541900
\end{array}
$$

Electronic Energy $(\mathrm{EE})=-1296.309895$ a.u.

$\mathrm{EE}+$ Thermal Enthalpy Correction $=-1296.014824$ a.u.

$\mathrm{EE}+$ Thermal Free Energy Correction $=-1296.075699$ a.u.

Imaginary Freq $=0$

\section{INT-I}

$\begin{array}{lrrr}\mathrm{C} & 3.85957100 & -1.71805800 & -0.18375300 \\ \mathrm{C} & 5.24058000 & -1.55149400 & -0.16777100 \\ \mathrm{C} & 5.79266800 & -0.27893500 & 0.00370700 \\ \mathrm{C} & 4.95364700 & 0.82910900 & 0.15888300 \\ \mathrm{C} & 3.56608000 & 0.69100800 & 0.14331900 \\ \mathrm{C} & 2.99992500 & -0.60784100 & -0.02956600 \\ \mathrm{H} & 3.42702900 & -2.70396500 & -0.31355900 \\ \mathrm{H} & 5.88692600 & -2.41439600 & -0.28831100 \\ \mathrm{H} & 6.86968700 & -0.14910100 & 0.01672300 \\ \mathrm{H} & 5.38372500 & 1.81785000 & 0.29241100 \\ \mathrm{C} & 2.74635100 & 1.91332700 & 0.30582100 \\ \mathrm{O} & 1.52952400 & 1.95151800 & 0.27510000 \\ \mathrm{H} & 3.33664100 & 2.83815000 & 0.45988700 \\ \mathrm{C} & 1.60575000 & -0.84339700 & -0.04436800 \\ \mathrm{C} & 0.42841000 & -1.16404300 & -0.05228100 \\ \mathrm{C} & -0.97056700 & -1.50748300 & -0.04596400 \\ \mathrm{H} & -1.21278700 & -2.14507000 & 0.81870500 \\ \mathrm{H} & -1.20195000 & -2.15291700 & -0.91409600 \\ \mathrm{~N} & -1.88813600 & -0.37372400 & -0.04919800 \\ \mathrm{C} & -1.36615900 & 0.94803700 & -0.38990700\end{array}$




$$
\begin{array}{llll}
\mathrm{H} & -0.64696300 & 1.23501300 & 0.39146700 \\
\mathrm{H} & -0.71722000 & 0.78827700 & -1.27556600 \\
\mathrm{C} & -2.34719600 & 2.04383900 & -0.66596800 \\
\mathrm{C} & -2.19016500 & 3.26760400 & -0.14928000 \\
\mathrm{H} & -3.14647500 & 1.83664600 & -1.37109100 \\
\mathrm{H} & -2.85820200 & 4.07906900 & -0.41894000 \\
\mathrm{H} & -1.38019200 & 3.50320700 & 0.53608600 \\
\mathrm{~S} & -3.62528700 & -0.81619300 & 0.01653700 \\
\mathrm{O} & -4.21488200 & -0.32633400 & -1.22148300 \\
\mathrm{O} & -3.59945700 & -2.23044400 & 0.36254300 \\
\mathrm{C} & -4.19751500 & 0.14961600 & 1.41684900 \\
\mathrm{H} & -5.27296900 & -0.03726400 & 1.47758900 \\
\mathrm{H} & -3.69441000 & -0.21110700 & 2.31422500 \\
\mathrm{H} & -4.00104800 & 1.20513300 & 1.22665600
\end{array}
$$

Electronic Energy $(\mathrm{EE})=-1220.701828$ a.u.

Electronic Energy in the solvent $\left(E_{S P}\right)=-1220.987896$ a.u.

Thermal Correction to Enthalpy $=0.282478$

Imaginary Freq = 0

\section{TS-Ia}

$\begin{array}{llrr}\mathrm{C} & 3.32032000 & 1.47471000 & -0.49253200 \\ \mathrm{C} & 4.64714400 & 1.82447700 & -0.22218000 \\ \mathrm{C} & 5.56341200 & 0.89995400 & 0.30028900 \\ \mathrm{C} & 5.16607400 & -0.40719800 & 0.56589100 \\ \mathrm{C} & 3.83976100 & -0.76567100 & 0.29832900 \\ \mathrm{C} & 2.91009200 & 0.16618100 & -0.23083800 \\ \mathrm{H} & 2.62859600 & 2.20373200 & -0.89849200 \\ \mathrm{H} & 4.97353500 & 2.83957800 & -0.42430600 \\ \mathrm{H} & 6.58454100 & 1.20704100 & 0.49712200 \\ \mathrm{H} & 5.86412100 & -1.13329400 & 0.97044100 \\ \mathrm{C} & 3.22029000 & -2.04526000 & 0.49355800\end{array}$




\begin{tabular}{|c|c|c|c|}
\hline $\mathrm{O}$ & 2.01445500 & -2.17367000 & 0.19165300 \\
\hline $\mathrm{H}$ & 3.74700400 & -2.91930600 & 0.89376500 \\
\hline $\mathrm{C}$ & 1.59979600 & -0.36453000 & -0.45107800 \\
\hline $\mathrm{C}$ & 0.39974500 & -0.34799000 & -0.81954600 \\
\hline $\mathrm{C}$ & -0.97650200 & -0.58314900 & -1.19573200 \\
\hline $\mathrm{H}$ & -1.16519700 & -1.67069000 & -1.10063400 \\
\hline $\mathrm{H}$ & -1.10961800 & -0.35262800 & -2.26709200 \\
\hline $\mathrm{N}$ & -1.92029400 & 0.12630700 & -0.32771100 \\
\hline $\mathrm{C}$ & -2.19074000 & 1.52967200 & -0.77773100 \\
\hline $\mathrm{H}$ & -1.20430100 & 1.98869200 & -0.89902700 \\
\hline $\mathrm{H}$ & -2.68373200 & 1.51209300 & -1.75961600 \\
\hline $\mathrm{C}$ & -3.00156200 & 2.35353400 & 0.18739700 \\
\hline $\mathrm{C}$ & -2.47488000 & 3.28776000 & 0.97965800 \\
\hline $\mathrm{H}$ & -4.07533800 & 2.18577700 & 0.17644400 \\
\hline $\mathrm{H}$ & -3.09501500 & 3.89354700 & 1.63275700 \\
\hline $\mathrm{H}$ & -1.40719800 & 3.49456100 & 0.99579500 \\
\hline $\mathrm{S}$ & -3.32445400 & -0.84108900 & 0.03729600 \\
\hline $\mathrm{O}$ & -4.49186600 & -0.25958700 & -0.62461500 \\
\hline $\mathrm{O}$ & -2.91090300 & -2.22806200 & -0.22217500 \\
\hline $\mathrm{C}$ & -3.48226200 & -0.62413600 & 1.81319000 \\
\hline $\mathrm{H}$ & -4.38147100 & -1.16820300 & 2.10895900 \\
\hline $\mathrm{H}$ & -2.59804000 & -1.04609900 & 2.28965200 \\
\hline $\mathrm{H}$ & -3.58218500 & 0.43889300 & 2.03053300 \\
\hline
\end{tabular}

Electronic Energy $(\mathrm{EE})=-1220.689894$ a.u.

Electronic Energy in the solvent $\left(E_{S P}\right)=-1220.980304$ a.u.

Thermal Correction to Enthalpy $=0.282668$

Imaginary Freq $=1$

\section{TS-Ib}
C
$3.48456000 \quad-1.57359700 \quad 0.15286100$
C
$\begin{array}{lll}4.82692500 & -1.47891000 & 0.52360100\end{array}$ 


\begin{tabular}{|c|c|c|c|}
\hline $\mathrm{C}$ & 5.45942100 & -0.23571100 & 0.66615900 \\
\hline $\mathrm{C}$ & 4.74910800 & 0.93613500 & 0.43740600 \\
\hline$C$ & 3.39567700 & 0.87020400 & 0.06409300 \\
\hline $\mathrm{C}$ & 2.75661800 & -0.40333300 & -0.07964100 \\
\hline $\mathrm{H}$ & 3.00340500 & -2.53848900 & 0.04167200 \\
\hline $\mathrm{H}$ & 5.39022400 & -2.38902300 & 0.70285100 \\
\hline $\mathrm{H}$ & 6.50387700 & -0.18998400 & 0.95389400 \\
\hline $\mathrm{H}$ & 5.23104100 & 1.90370100 & 0.54398700 \\
\hline$C$ & 2.63596500 & 2.06792900 & -0.18042000 \\
\hline $\mathrm{O}$ & 1.43352600 & 2.11863700 & -0.51651900 \\
\hline $\mathrm{H}$ & 3.14351400 & 3.03630800 & -0.06507300 \\
\hline$C$ & 1.39867100 & -0.34396100 & -0.46610000 \\
\hline$C$ & 0.41612900 & 0.38639000 & -0.75410100 \\
\hline $\mathrm{C}$ & -0.91929000 & 0.81009300 & -1.18290200 \\
\hline $\mathrm{H}$ & -1.09527800 & 0.41547700 & -2.19501700 \\
\hline $\mathrm{H}$ & -0.90282300 & 1.90108200 & -1.26290400 \\
\hline $\mathrm{N}$ & -1.97561300 & 0.45726000 & -0.21410500 \\
\hline$C$ & -3.19168100 & 1.32342700 & -0.40318200 \\
\hline $\mathrm{H}$ & -2.83211000 & 2.34706500 & -0.26367500 \\
\hline $\mathrm{H}$ & -3.56113700 & 1.22243500 & -1.43280500 \\
\hline $\mathrm{C}$ & -4.31490900 & 1.05859700 & 0.56392100 \\
\hline $\mathrm{C}$ & -4.57529500 & 1.83953500 & 1.61323700 \\
\hline $\mathrm{H}$ & -4.96100700 & 0.21501300 & 0.33441800 \\
\hline $\mathrm{H}$ & -5.42383100 & 1.65038800 & 2.26326400 \\
\hline $\mathrm{H}$ & -3.96256400 & 2.70526800 & 1.85298600 \\
\hline S & -2.27045600 & -1.25064000 & -0.21832000 \\
\hline $\mathrm{O}$ & -3.48900400 & -1.56619400 & -0.96375600 \\
\hline $\mathrm{O}$ & -0.97980100 & -1.84123200 & -0.63026800 \\
\hline$C$ & -2.52072000 & -1.60764300 & 1.52338600 \\
\hline $\mathrm{H}$ & -2.74095900 & -2.67517000 & 1.58553500 \\
\hline $\mathrm{H}$ & -1.60274500 & -1.36423800 & 2.05710900 \\
\hline
\end{tabular}


Electronic Energy $(\mathrm{EE})=-1220.686334$ a.u.

Electronic Energy in the solvent $\left(E_{S P}\right)=-1220.972578$ a.u.

Thermal Correction to Enthalpy $=0.283419$

Imaginary Freq $=1$

\section{INT-II}

C

$3.20601200 \quad 1.61755000-0.06580000$

$\begin{array}{llll}\text { C } & 4.51380600 & 1.92202100 & 0.30973800\end{array}$

$\begin{array}{llll}\text { C } & 5.47788300 & 0.92273700 & 0.58394500\end{array}$

C $\quad 5.15663900 \quad-0.41841100 \quad 0.48928400$

C $\quad 3.83473000 \quad-0.74035300 \quad 0.11002800$

$\begin{array}{llll}\text { C } & 2.86538000 & 0.26996200 & -0.16576600\end{array}$

$\begin{array}{llll}\mathrm{H} & 2.48603500 & 2.40163600 & -0.27069700\end{array}$

$\begin{array}{llll}\mathrm{H} & 4.80608700 & 2.96391800 & 0.39562900\end{array}$

$\begin{array}{llll}\mathrm{H} & 6.48009500 & 1.22076300 & 0.87132200\end{array}$

$\mathrm{H} \quad 5.88652400 \quad-1.19364200 \quad 0.69646700$

C $\quad 3.17737400 \quad-1.96368300 \quad-0.07657200$

O $\quad 1.92712100 \quad-1.82028300 \quad-0.43516300$

$\mathrm{H} \quad 3.53227900 \quad-2.98347600 \quad 0.02801400$

C $\quad 1.63990800 \quad-0.41116400 \quad-0.51698900$

C $\quad 0.42387100 \quad-0.02312400 \quad-0.87230300$

C $\quad-0.90511500-0.51212900-1.24178200$

$\mathrm{H} \quad-0.93872200 \quad-1.60162300 \quad-1.07087700$

$\mathrm{H} \quad-1.08202700 \quad-0.34755800 \quad-2.31665000$

$\mathrm{N} \quad \quad-1.92594100 \quad 0.13520700 \quad-0.40417700$

$\begin{array}{llll}\text { C } & -2.52231100 & 1.34804300 & -1.04751600\end{array}$

$\mathrm{H} \quad-1.66740200 \quad 1.96082500 \quad-1.35078500$

$\mathrm{H} \quad-3.06564900 \quad 1.04753500 \quad-1.95472800$

$\begin{array}{lllll}\text { C } & -3.42329200 & 2.16312800 & -0.15856700\end{array}$

$\begin{array}{llll}\text { C } & -3.06932100 & 3.33606100 & 0.36753400\end{array}$ 


$$
\begin{array}{llll}
\mathrm{H} & -4.42533800 & 1.77222700 & -0.00210800 \\
\mathrm{H} & -3.76002300 & 3.92140400 & 0.96626800 \\
\mathrm{H} & -2.08283800 & 3.76476900 & 0.20649400 \\
\mathrm{~S} & -3.04069800 & -0.99277900 & 0.30779800 \\
\mathrm{O} & -4.35819000 & -0.81111200 & -0.30155600 \\
\mathrm{O} & -2.35842200 & -2.29341900 & 0.27602200 \\
\mathrm{C} & -3.10217900 & -0.43726400 & 2.01541600 \\
\mathrm{H} & -3.83769000 & -1.06947100 & 2.51661300 \\
\mathrm{H} & -2.11370100 & -0.56889400 & 2.45410000 \\
\mathrm{H} & -3.40926000 & 0.60786300 & 2.03708500
\end{array}
$$

Electronic Energy $(\mathrm{EE})=-1220.702926$ a.u.

Electronic Energy in the solvent $\left(E_{S P}\right)=-1220.990761$ a.u.

Thermal Correction to Enthalpy $=0.285266$

Imaginary Freq $=0$

\section{INT-III}

$\begin{array}{lrrr}\mathrm{C} & -3.07743400 & -1.45589600 & -0.13048800 \\ \mathrm{C} & -4.44790100 & -1.69961800 & -0.17980000 \\ \mathrm{C} & -5.39188500 & -0.64975400 & -0.14229900 \\ \mathrm{C} & -4.99254500 & 0.67573400 & -0.04911400 \\ \mathrm{C} & -3.60843900 & 0.94137300 & 0.00324100 \\ \mathrm{C} & -2.64554400 & -0.12280000 & -0.04239100 \\ \mathrm{H} & -2.37977200 & -2.28484900 & -0.15733600 \\ \mathrm{H} & -4.80122800 & -2.72299800 & -0.24874700 \\ \mathrm{H} & -6.44896400 & -0.88935200 & -0.18547900 \\ \mathrm{H} & -5.71888000 & 1.48063000 & -0.01675200 \\ \mathrm{C} & -2.87520800 & 2.12345000 & 0.10706300 \\ \mathrm{O} & -1.54900900 & 1.84599900 & 0.12687200 \\ \mathrm{H} & -3.14481900 & 3.16767100 & 0.17032300 \\ \mathrm{C} & -1.36423600 & 0.49669400 & 0.03340500 \\ \mathrm{C} & -0.01033600 & 0.04438800 & 0.01968300\end{array}$




$\begin{array}{lrrr}\mathrm{C} & 1.13863700 & 1.03332700 & 0.26034300 \\ \mathrm{H} & 0.85192700 & 1.74172600 & 1.04533400 \\ \mathrm{H} & 1.42088800 & 1.59701000 & -0.63028500 \\ \mathrm{~N} & 2.26679400 & 0.20940700 & 0.71804300 \\ \mathrm{C} & 1.75325400 & -1.00552300 & 1.37567000 \\ \mathrm{H} & 2.45542500 & -1.83351200 & 1.25226600 \\ \mathrm{H} & 1.60664200 & -0.81844700 & 2.44504200 \\ \mathrm{C} & 0.41204600 & -1.30836700 & 0.71048600 \\ \mathrm{C} & 0.34916200 & -1.25644300 & -0.75532300 \\ \mathrm{H} & -0.33803000 & -1.85351500 & 1.27551800 \\ \mathrm{H} & -0.47134300 & -1.74400100 & -1.26941900 \\ \mathrm{H} & 1.27650000 & -1.21151900 & -1.32063100 \\ \mathrm{~S} & 3.60483600 & 0.03484700 & -0.35686700 \\ \mathrm{O} & 3.69886500 & -1.37473700 & -0.75700200 \\ \mathrm{O} & 3.46500700 & 1.09406300 & -1.35774200 \\ \mathrm{C} & 4.99791500 & 0.41345100 & 0.71000800 \\ \mathrm{H} & 5.01370700 & -0.29641000 & 1.53764100 \\ \mathrm{H} & 5.89619200 & 0.30380000 & 0.09947300 \\ \mathrm{H} & 4.89299600 & 1.43768100 & 1.06669100\end{array}$

Electronic Energy $(\mathrm{EE})=-1220.787202$ a.u.

Electronic Energy in the solvent $\left(E_{S P}\right)=-1221.067608$ a.u.

Thermal Correction to Enthalpy $=0.287658$

Imaginary Freq $=0$

\section{INT-III+O2}
C
$\begin{array}{lll}-2.80279600 & -1.65559000 & -0.67150800\end{array}$
C
$\begin{array}{lll}-4.16196800 & -1.93476900 & -0.78837200\end{array}$
C
$\begin{array}{lll}-5.14202400 & -1.13663600 & -0.15779200\end{array}$
C
$\begin{array}{lll}-4.79035800 & -0.03742100 & 0.61196300\end{array}$
C
$\begin{array}{lll}-3.41815100 & 0.25953300 & 0.74195200\end{array}$
C
$\begin{array}{lll}-2.41987000 & -0.54476200 & 0.09728900\end{array}$ 


\begin{tabular}{|c|c|c|c|}
\hline $\mathrm{H}$ & -2.07609100 & -2.29121700 & -1.16386800 \\
\hline $\mathrm{H}$ & -4.47809200 & -2.78857100 & -1.37829300 \\
\hline $\mathrm{H}$ & -6.18893800 & -1.39367400 & -0.27866400 \\
\hline $\mathrm{H}$ & -5.54394400 & 0.57231700 & 1.09853000 \\
\hline $\mathrm{C}$ & -2.72717100 & 1.25791600 & 1.43046600 \\
\hline $\mathrm{O}$ & -1.39258600 & 1.10346100 & 1.25401400 \\
\hline $\mathrm{H}$ & -3.03381000 & 2.08371200 & 2.05539900 \\
\hline $\mathrm{C}$ & -1.16122900 & 0.02968300 & 0.44365000 \\
\hline $\mathrm{C}$ & 0.20373700 & -0.26447300 & 0.15298500 \\
\hline $\mathrm{C}$ & 1.33619800 & 0.52800800 & 0.82246600 \\
\hline $\mathrm{H}$ & 1.06455800 & 0.76142600 & 1.85736500 \\
\hline $\mathrm{H}$ & 1.55455000 & 1.45880500 & 0.29489400 \\
\hline $\mathrm{N}$ & 2.50605500 & -0.36506100 & 0.79821000 \\
\hline $\mathrm{C}$ & 2.05344100 & -1.76687600 & 0.76660500 \\
\hline $\mathrm{H}$ & 2.79753200 & -2.39036800 & 0.26642100 \\
\hline $\mathrm{H}$ & 1.91348900 & -2.13652800 & 1.78853800 \\
\hline $\mathrm{C}$ & 0.71574700 & -1.75292600 & 0.03466800 \\
\hline $\mathrm{C}$ & 0.58246400 & -0.94800300 & -1.18795200 \\
\hline $\mathrm{H}$ & 0.00787800 & -2.55129700 & 0.23632400 \\
\hline $\mathrm{H}$ & -0.23635600 & -1.15641000 & -1.86722800 \\
\hline $\mathrm{H}$ & 1.46054700 & -0.51049800 & -1.65747700 \\
\hline S & 3.81684100 & 0.06659800 & -0.23812100 \\
\hline $\mathrm{O}$ & 4.42910200 & -1.18862800 & -0.67441500 \\
\hline $\mathrm{O}$ & 3.32679600 & 1.04978600 & -1.21353700 \\
\hline $\mathrm{C}$ & 4.93597100 & 0.90088900 & 0.89073000 \\
\hline $\mathrm{H}$ & 5.23518100 & 0.19449600 & 1.66457500 \\
\hline $\mathrm{H}$ & 5.79718100 & 1.21698400 & 0.29905300 \\
\hline $\mathrm{H}$ & 4.42997300 & 1.76766300 & 1.31700100 \\
\hline $\mathrm{O}$ & -1.55775800 & 2.37036900 & -1.57063300 \\
\hline $\mathrm{O}$ & -2.37298400 & 3.04571100 & -0.97455200 \\
\hline
\end{tabular}

Electronic Energy $(\mathrm{EE})=-1371.109265$ a.u. 
Electronic Energy in the solvent $\left(\mathrm{E}_{\mathrm{SP}}\right)=-1371.438121$ a.u.

Thermal Correction to Enthalpy $=0.296162$

Imaginary Freq $=0$

TS2

C $\quad-2.84929400 \quad-1.47759100 \quad-0.82730100$

C $\quad-4.20570400 \quad-1.83361100 \quad-0.80540500$

C $\quad-5.16909800 \quad-1.08850700 \quad-0.09962000$

$\begin{array}{llll}\text { C } & -4.80610000 & 0.05192200 & 0.60397300\end{array}$

$\begin{array}{llll}\text { C } & -3.44350000 & 0.39698500 & 0.60255200\end{array}$

$\begin{array}{llll}\text { C } & -2.46532900 & -0.36994900 & -0.08519000\end{array}$

H $\quad-2.14591900 \quad-2.04983700 \quad-1.41862900$

$\mathrm{H} \quad-4.52464600 \quad-2.71050700 \quad-1.35995700$

$\mathrm{H} \quad-6.20589700 \quad-1.40571900 \quad-0.11920900$

$\begin{array}{llll}\mathrm{H} & -5.54019900 & 0.65050500 & 1.13271600\end{array}$

$\begin{array}{llll}\text { C } & -2.74829500 & 1.48510900 & 1.16878300\end{array}$

$\begin{array}{llll}\mathrm{O} & -1.45270500 & 1.39167800 & 1.01654600\end{array}$

$\mathrm{H} \quad-3.10904200 \quad 2.32262600 \quad 1.75719200$

$\begin{array}{llll}\text { C } & -1.19184200 & 0.38253200 & 0.02501000\end{array}$

$\begin{array}{llll}\text { C } & 0.18419700 & -0.12660300 & 0.07248400\end{array}$

$\begin{array}{llll}\text { C } & 1.32959200 & 0.90310500 & -0.04271700\end{array}$

$\begin{array}{llll}\mathrm{H} & 1.03798200 & 1.83976400 & 0.44613500\end{array}$

$\begin{array}{llll}\mathrm{H} & 1.59766600 & 1.11159900 & -1.07960500\end{array}$

$\begin{array}{llll}\mathrm{N} & 2.46553300 & 0.29996500 & 0.67097100\end{array}$

$\begin{array}{llll}\text { C } & 1.95765200 & -0.63770900 & 1.69058400\end{array}$

$\mathrm{H} \quad 2.68165500 \quad-1.43590500 \quad 1.85990200$

$\begin{array}{llll}\mathrm{H} & 1.79579200 & -0.10437500 & 2.63470000\end{array}$

C $\quad 0.62516400 \quad-1.13760500 \quad 1.14142500$

C $\quad 0.55241700 \quad-1.55430500 \quad-0.29234900$

$\begin{array}{llll}\mathrm{H} & -0.09752700 & -1.55056400 & 1.83902500\end{array}$

$\mathrm{H} \quad-0.23669400 \quad-2.24864500 \quad-0.55180800$ 


$\begin{array}{lrrr}\mathrm{H} & 1.45827500 & -1.67159400 & -0.87967800 \\ \mathrm{~S} & 3.81464100 & -0.20805500 & -0.27474600 \\ \mathrm{O} & 4.38606800 & -1.37257100 & 0.40239700 \\ \mathrm{O} & 3.39497100 & -0.28010900 & -1.68073100 \\ \mathrm{C} & 4.94364000 & 1.17726000 & -0.09828400 \\ \mathrm{H} & 5.19516700 & 1.28649800 & 0.95635400 \\ \mathrm{H} & 5.83103100 & 0.93409000 & -0.68565000 \\ \mathrm{H} & 4.46560300 & 2.07660000 & -0.48784700 \\ \mathrm{O} & -1.26035200 & 1.38362300 & -1.32950100 \\ \mathrm{O} & -2.16513800 & 2.27217800 & -1.24223300\end{array}$

Electronic Energy $(\mathrm{EE})=-1371.090724$ a.u.

Electronic Energy in the solvent $\left(E_{S P}\right)=-1371.422664$ a.u.

Thermal Correction to Enthalpy $=0.296148$

Imaginary Freq $=1$

\section{INT-IV}

C

$\begin{array}{lll}-2.90017600 & -0.97793600 & -1.28637400\end{array}$

C $\quad-4.16730200-1.56872600 \quad-1.12917100$

C $\quad-4.97644300 \quad-1.32422300 \quad-0.00639500$

C $\quad-4.54967200 \quad-0.46131200 \quad 0.99607600$

$\begin{array}{llll}\text { C } & -3.28494100 & 0.13079500 & 0.83576700\end{array}$

C $\quad-2.45994000-0.13859200 \quad-0.28123900$

$\mathrm{H} \quad-2.31114700-1.16734800 \quad-2.17642600$

$\mathrm{H} \quad-4.53451200 \quad-2.23172900-1.90641000$

$\mathrm{H} \quad-5.94539700 \quad-1.80518600 \quad 0.06798300$

$\mathrm{H} \quad-5.16904000 \quad-0.24660000 \quad 1.86071600$

C $\quad-2.59871000 \quad 1.08689800 \quad 1.61667500$

$\begin{array}{lllll}\mathrm{O} & -1.45683300 & 1.43804000 & 1.14304500\end{array}$

$\mathrm{H} \quad-2.90379100 \quad 1.55129400 \quad 2.55239000$

C $\quad-1.19853000 \quad 0.67549100 \quad-0.16364200$

C $\quad 0.14191700 \quad 0.03441300 \quad-0.06238000$ 


\begin{tabular}{lrrr}
$\mathrm{C}$ & 1.35917600 & 0.97651300 & 0.05375200 \\
$\mathrm{H}$ & 1.10198600 & 1.84236000 & 0.67484100 \\
$\mathrm{H}$ & 1.71035800 & 1.32883600 & -0.91718600 \\
$\mathrm{~N}$ & 2.39784600 & 0.17383100 & 0.71933800 \\
$\mathrm{C}$ & 1.75745500 & -0.87825300 & 1.53245100 \\
$\mathrm{H}$ & 2.40981300 & -1.74974600 & 1.59942300 \\
$\mathrm{H}$ & 1.57358700 & -0.50014600 & 2.54527200 \\
$\mathrm{C}$ & 0.42994000 & -1.16370000 & 0.83491100 \\
$\mathrm{C}$ & 0.43184900 & -1.33757700 & -0.65219700 \\
$\mathrm{H}$ & -0.36220800 & -1.63456000 & 1.41032900 \\
$\mathrm{H}$ & -0.38953700 & -1.90349400 & -1.07505600 \\
$\mathrm{H}$ & 1.36583400 & -1.42899600 & -1.19884300 \\
$\mathrm{~S}$ & 3.77101800 & -0.27722400 & -0.22148200 \\
$\mathrm{O}$ & 4.22678400 & -1.56728800 & 0.29746200 \\
$\mathrm{O}$ & 3.43870300 & -0.10783600 & -1.64234700 \\
$\mathrm{C}$ & 4.96562800 & 0.98563300 & 0.22856000 \\
$\mathrm{H}$ & 5.15890800 & 0.91275900 & 1.29848600 \\
$\mathrm{H}$ & 5.87101200 & 0.77791800 & -0.34478900 \\
$\mathrm{H}$ & 4.56487200 & 1.96438800 & -0.03717700 \\
$\mathrm{O}$ & -1.10505800 & 1.71920700 & -1.16113300 \\
$\mathrm{O}$ & -2.22402600 & 2.42693600 & -1.25527500 \\
\hline & & & \\
$\mathrm{H}$ & & &
\end{tabular}

Electronic Energy $(\mathrm{EE})=-1371.093600$ a.u.

Electronic Energy in the solvent $\left(E_{S P}\right)=-1371.428304$ a.u.

Thermal Correction to Enthalpy $=0.297195$

Imaginary Freq $=0$

\section{TS-III}
C
$2.46309600 \quad-0.85800800 \quad 1.41523300$
$\begin{array}{llll}\text { O } & 1.57630300 & 0.14874200 & 1.86129900\end{array}$
O $\quad 0.34395100 \quad-0.40781200 \quad 2.21970100$
$\begin{array}{llll}\mathrm{O} & -0.87189700 & 0.35378500 & 1.48998300\end{array}$ 


\begin{tabular}{|c|c|c|c|}
\hline $\mathrm{O}$ & -1.43137300 & 1.71994300 & 0.77296600 \\
\hline $\mathrm{C}$ & -2.77314200 & 1.61389600 & 0.55062800 \\
\hline $\mathrm{C}$ & 1.86287900 & -1.97141000 & 0.58429700 \\
\hline $\mathrm{C}$ & 2.06135700 & -3.19750000 & 1.26765700 \\
\hline $\mathrm{C}$ & 1.24125100 & -1.95330500 & -0.64687000 \\
\hline $\mathrm{C}$ & 1.65320700 & -4.43366800 & 0.72774600 \\
\hline $\mathrm{C}$ & 0.81572600 & -3.18606800 & -1.17673500 \\
\hline $\mathrm{H}$ & 1.06976300 & -1.03321600 & -1.19337100 \\
\hline $\mathrm{C}$ & 1.02410300 & -4.40620000 & -0.50754200 \\
\hline $\mathrm{H}$ & 1.82389400 & -5.36451900 & 1.25853400 \\
\hline $\mathrm{H}$ & 0.30368200 & -3.19744300 & -2.13247700 \\
\hline $\mathrm{H}$ & 0.67951800 & -5.32668300 & -0.96413900 \\
\hline $\mathrm{C}$ & -3.23566600 & 2.99436000 & 0.11162600 \\
\hline $\mathrm{C}$ & -2.88779100 & 3.74138700 & -0.99895900 \\
\hline $\mathrm{C}$ & -4.08784200 & 3.54586400 & 1.09856000 \\
\hline $\mathrm{C}$ & -3.45589800 & 5.02160500 & -1.13091000 \\
\hline $\mathrm{H}$ & -2.19057900 & 3.37437600 & -1.74277700 \\
\hline $\mathrm{C}$ & -4.65504000 & 4.82443100 & 0.96759500 \\
\hline $\mathrm{C}$ & -4.33143800 & 5.55463400 & -0.17130300 \\
\hline $\mathrm{H}$ & -3.20136200 & 5.61956600 & -2.00050500 \\
\hline $\mathrm{H}$ & -5.31308900 & 5.22864900 & 1.73010600 \\
\hline $\mathrm{H}$ & -4.74386600 & 6.54714000 & -0.31530300 \\
\hline $\mathrm{C}$ & 2.67226900 & -2.87547800 & 2.49097000 \\
\hline $\mathrm{O}$ & 2.89693200 & -1.61154600 & 2.65815800 \\
\hline $\mathrm{H}$ & 2.97366900 & -3.53198200 & 3.30439100 \\
\hline $\mathrm{C}$ & -4.16575900 & 2.60970700 & 2.15806300 \\
\hline $\mathrm{O}$ & -3.48309400 & 1.54459200 & 1.98349000 \\
\hline $\mathrm{H}$ & -4.72365500 & 2.69889000 & 3.08996800 \\
\hline $\mathrm{C}$ & 3.66360100 & -0.15582700 & 0.83423800 \\
\hline $\mathrm{C}$ & 3.39970100 & 0.75379500 & -0.37997000 \\
\hline $\mathrm{C}$ & 4.72420300 & 0.52943300 & 1.68355300 \\
\hline
\end{tabular}




\begin{tabular}{|c|c|c|c|}
\hline $\mathrm{C}$ & 5.05562300 & -0.74447300 & 0.96033400 \\
\hline $\mathrm{H}$ & 2.45831000 & 1.29825600 & -0.23086400 \\
\hline $\mathrm{H}$ & 3.35555300 & 0.22707100 & -1.33498200 \\
\hline $\mathrm{N}$ & 4.52198700 & 1.70316200 & -0.39208700 \\
\hline $\mathrm{C}$ & 5.07735400 & 1.83274400 & 0.97557300 \\
\hline $\mathrm{H}$ & 4.66554800 & 0.52237400 & 2.76689800 \\
\hline $\mathrm{H}$ & 5.18474200 & -1.63085900 & 1.57143600 \\
\hline $\mathrm{H}$ & 5.70444300 & -0.70259000 & 0.09038100 \\
\hline$S$ & 5.64040400 & 1.54168100 & -1.69908900 \\
\hline $\mathrm{H}$ & 6.15606400 & 1.99985200 & 0.93393600 \\
\hline $\mathrm{H}$ & 4.60822300 & 2.68278600 & 1.48347000 \\
\hline $\mathrm{O}$ & 6.89045800 & 0.95354900 & -1.20434100 \\
\hline $\mathrm{O}$ & 4.88671000 & 0.90306400 & -2.78196300 \\
\hline $\mathrm{C}$ & 5.95668700 & 3.25593600 & -2.12626400 \\
\hline $\mathrm{H}$ & 6.37780800 & 3.76782700 & -1.26017800 \\
\hline $\mathrm{H}$ & 6.68178500 & 3.24145200 & -2.94230300 \\
\hline $\mathrm{H}$ & 5.02097000 & 3.71400100 & -2.44453600 \\
\hline $\mathrm{C}$ & -3.27275400 & 0.39794600 & -0.18616200 \\
\hline $\mathrm{C}$ & -3.14607600 & -0.98295800 & 0.48594200 \\
\hline $\mathrm{C}$ & -4.56569400 & 0.37396300 & -0.99219600 \\
\hline $\mathrm{C}$ & -3.24918900 & 0.40947500 & -1.70658900 \\
\hline $\mathrm{H}$ & -3.42191800 & -0.90891500 & 1.54257400 \\
\hline $\mathrm{H}$ & -2.14965300 & -1.42021500 & 0.42661200 \\
\hline $\mathrm{N}$ & -4.11232400 & -1.83737200 & -0.22154100 \\
\hline $\mathrm{C}$ & -5.19234000 & -1.00205500 & -0.79702700 \\
\hline $\mathrm{H}$ & -5.22316200 & 1.23583200 & -1.04653100 \\
\hline $\mathrm{H}$ & -2.98786100 & 1.33315500 & -2.20853200 \\
\hline $\mathrm{H}$ & -2.90641100 & -0.48499300 & -2.21822800 \\
\hline $\mathrm{S}$ & -3.48477900 & -3.11088100 & -1.18057900 \\
\hline $\mathrm{H}$ & -5.54976800 & -1.42830200 & -1.73718700 \\
\hline $\mathrm{H}$ & -6.03013000 & -0.95282600 & -0.09257600 \\
\hline
\end{tabular}




$$
\begin{array}{llrr}
\mathrm{O} & -3.59099400 & -2.77583800 & -2.60585000 \\
\mathrm{O} & -2.16729000 & -3.43316100 & -0.60722800 \\
\mathrm{C} & -4.62839000 & -4.44493500 & -0.81952200 \\
\mathrm{H} & -5.63372000 & -4.13796300 & -1.11094800 \\
\mathrm{H} & -4.31195700 & -5.30027800 & -1.41956600 \\
\mathrm{H} & -4.57635700 & -4.67005100 & 0.24526000
\end{array}
$$

Electronic Energy $(\mathrm{EE})=-2742.081351$ a.u.

Electronic Energy in the solvent $\left(E_{S P}\right)=-2742.790765$ a.u.

Thermal Correction to Enthalpy $=0.593562$

Imaginary Freq $=1$

\section{INT-V}

$\begin{array}{llll}\mathrm{C} & -3.35168300 & 1.42966400 & -0.59184400 \\ \mathrm{C} & -4.66350400 & 1.55487500 & -0.11883800 \\ \mathrm{C} & -5.29567300 & 0.48227000 & 0.50988900 \\ \mathrm{C} & -4.61943200 & -0.72866500 & 0.66269200 \\ \mathrm{C} & -3.30575300 & -0.86639900 & 0.20238900 \\ \mathrm{C} & -2.66336900 & 0.23129700 & -0.40999100 \\ \mathrm{H} & -2.87449000 & 2.26220200 & -1.09900500 \\ \mathrm{H} & -5.19229000 & 2.49246000 & -0.25654600 \\ \mathrm{H} & -6.31624700 & 0.58315700 & 0.86340800 \\ \mathrm{H} & -5.11320700 & -1.57874600 & 1.12536100 \\ \mathrm{C} & -2.60391600 & -2.16080300 & 0.29278500 \\ \mathrm{O} & -1.47923500 & -2.33010000 & -0.15250200 \\ \mathrm{H} & -3.15155500 & -2.98545500 & 0.78544700 \\ \mathrm{C} & -1.25558100 & 0.10900200 & -0.92837300 \\ \mathrm{C} & -0.07700100 & 0.12240900 & 0.00429000 \\ \mathrm{C} & 1.19229500 & -0.32794400 & -0.69321700 \\ \mathrm{H} & 1.03563000 & -0.09871600 & -1.77707200 \\ \mathrm{H} & 1.42230900 & -1.39516800 & -0.61036900 \\ \mathrm{~N} & 2.23954700 & 0.47861800 & -0.13205800\end{array}$




$\begin{array}{lrrr}\mathrm{C} & 1.75270500 & 1.66599200 & 0.57743500 \\ \mathrm{H} & 2.33945800 & 1.79874100 & 1.49516300 \\ \mathrm{H} & 1.92929100 & 2.55894100 & -0.04213400 \\ \mathrm{C} & 0.28224800 & 1.40184300 & 0.80991800 \\ \mathrm{C} & -0.10669000 & 0.12662100 & 1.49937500 \\ \mathrm{H} & -0.39319900 & 2.24967600 & 0.83448100 \\ \mathrm{H} & -1.08532400 & 0.12962300 & 1.96880300 \\ \mathrm{H} & 0.64488300 & -0.45925700 & 2.02282500 \\ \mathrm{~S} & 3.88410300 & -0.18150300 & 0.20268500 \\ \mathrm{O} & 4.45187800 & 0.76980200 & 1.14422300 \\ \mathrm{O} & 3.65663700 & -1.58473100 & 0.51266000 \\ \mathrm{C} & 4.66474400 & -0.01526800 & -1.40473100 \\ \mathrm{H} & 4.69463000 & 1.04095200 & -1.67387500 \\ \mathrm{H} & 5.67781500 & -0.40737000 & -1.28185700 \\ \mathrm{H} & 4.11490900 & -0.61312800 & -2.13260100 \\ \mathrm{O} & -1.03570800 & 0.12333400 & -2.13078900\end{array}$

Electronic Energy $(\mathrm{EE})=-1295.977994$ a.u.

Electronic Energy in the solvent $\left(\mathrm{E}_{\mathrm{SP}}\right)=-1296.29022$ a.u.

Thermal Correction to Enthalpy $=0.28943$

Imaginary Freq $=0$

\section{TS-IV}

$\begin{array}{lrrr}\mathrm{C} & -3.88563400 & 1.10786800 & -0.28916300 \\ \mathrm{C} & -5.11995300 & 0.45907600 & -0.20046900 \\ \mathrm{C} & -5.17738900 & -0.92337900 & -0.02525700 \\ \mathrm{C} & -3.99413800 & -1.65841000 & 0.06166100 \\ \mathrm{C} & -2.74889200 & -1.02120100 & -0.00897000 \\ \mathrm{C} & -2.68894500 & 0.38803700 & -0.18862500 \\ \mathrm{H} & -3.83782100 & 2.18072300 & -0.43814600 \\ \mathrm{H} & -6.03520800 & 1.03702800 & -0.27448400 \\ \mathrm{H} & -6.13541500 & -1.42826800 & 0.03823400\end{array}$




$\begin{array}{lrrr}\mathrm{H} & -4.03284400 & -2.73699200 & 0.18363200 \\ \mathrm{C} & -1.55987300 & -1.86293000 & 0.07672300 \\ \mathrm{O} & -0.39957500 & -1.45047100 & 0.08719600 \\ \mathrm{H} & -1.72998500 & -2.95059100 & 0.14133700 \\ \mathrm{C} & -1.44721500 & 1.18335800 & -0.32463700 \\ \mathrm{C} & -0.04252300 & 0.78191600 & 0.01589800 \\ \mathrm{C} & 1.01593900 & 0.62204600 & -1.05110900 \\ \mathrm{H} & 0.82255100 & 1.43926500 & -1.76462400 \\ \mathrm{H} & 0.98633400 & -0.32604900 & -1.59099900 \\ \mathrm{~N} & 2.29833700 & 0.82594500 & -0.37995200 \\ \mathrm{C} & 2.05225600 & 1.65237700 & 0.81274800 \\ \mathrm{H} & 2.79121100 & 1.45766200 & 1.59053700 \\ \mathrm{H} & 2.08932800 & 2.71024100 & 0.53390100 \\ \mathrm{C} & 0.61173400 & 1.29530200 & 1.27152800 \\ \mathrm{C} & 0.49785500 & 0.04765500 & 2.07198000 \\ \mathrm{H} & 0.05959000 & 2.13084700 & 1.71263300 \\ \mathrm{H} & -0.40359300 & -0.14550700 & 2.64254200 \\ \mathrm{H} & 1.27423400 & -0.70774400 & 2.01788100 \\ \mathrm{H} & 3.30932900 & -0.57383900 & -0.21118900 \\ \mathrm{H} & 3.42386300 & -0.89554400 & 1.21766100 \\ \mathrm{H} & 2.80096000 & -1.55123300 & -1.17370200 \\ \mathrm{H} & 4.89325800 & 0.05052200 & -0.77714500 \\ \mathrm{H} & 5.19639500 & 0.88079700 & -0.13848500 \\ \mathrm{H} & 5.60119600 & -0.77571200 & -0.68750900 \\ \mathrm{H} & 4.79001300 & 0.36349400 & -1.81574800 \\ \mathrm{H} & -1.43931000 & 2.33939800 & -0.72903000 \\ \mathrm{H} & & & \\ \mathrm{H} & & & \end{array}$

Electronic Energy (EE) = -1295.946620 a.u.

Electronic Energy in the solvent $\left(E_{S P}\right)=-1296.255466$ a.u.

Thermal Correction to Enthalpy $=0.288465$

Imaginary Freq = 1 


\section{INT-VI}

C

$\begin{array}{lll}-3.88126100 & 0.64706800 & -0.77883800\end{array}$

C $\quad-4.95645800 \quad-0.21171500 \quad-0.51828700$

C $\quad-4.76822000-1.43423600 \quad 0.14486400$

C $\quad-3.49698700 \quad-1.81426700 \quad 0.55449300$

C $\quad-2.40656600 \quad-0.95358800 \quad 0.29940000$

$\begin{array}{lllll}\text { C } & -2.60310400 & 0.28498900 & -0.36995900\end{array}$

$\mathrm{H} \quad-4.02355400 \quad 1.59247100 \quad-1.29096700$

$\mathrm{H} \quad \begin{aligned} & \mathrm{H} \\ & \mathrm{H}\end{aligned}$

$\mathrm{H} \quad-5.61627100 \quad-2.08322300 \quad 0.33381000$

$\mathrm{H} \quad-3.33658700 \quad-2.76103400 \quad 1.06130500$

C $\quad-1.09462000 \quad-1.33034900 \quad 0.66626600$

O $\quad-0.04930400 \quad-0.62490500 \quad 0.45438700$

$\mathrm{H} \quad-0.87486500 \quad-2.28874800 \quad 1.13568500$

C $\quad-1.44102300 \quad 1.17429100 \quad-0.61030900$

C $\quad-0.07718000 \quad 0.73727100 \quad-0.10212500$

C $\quad \begin{array}{llll}1.04594400 & 0.77042600 & -1.16961000\end{array}$

$\mathrm{H} \quad 0.92906400 \quad 1.71487600 \quad-1.71178500$

$\mathrm{H} \quad 0.99731700 \quad-0.06346500 \quad-1.86960500$

$\mathrm{N} \quad 2.28632200 \quad 0.76310800 \quad-0.40927500$

$\begin{array}{llll}\mathrm{C} & 2.06648500 & 1.54111500 & 0.82630400\end{array}$

$\mathrm{H} \quad 2.52240200 \quad 1.03003100 \quad 1.67504600$

$\begin{array}{llll}\mathrm{H} & 2.51629200 & 2.53341700 & 0.72970100\end{array}$

$\begin{array}{llll}\text { C } & 0.51414100 & 1.70008000 & 1.00873400\end{array}$

$\begin{array}{llll}\text { C } & 0.03035000 & 1.48482200 & 2.39106100\end{array}$

$\begin{array}{llll}\mathrm{H} & 0.20519300 & 2.68913600 & 0.65396900\end{array}$

$\mathrm{H} \quad-0.67579600 \quad 2.16576500 \quad 2.85127900$

$\begin{array}{llll}\mathrm{H} & 0.42358000 & 0.66872300 & 2.98832300\end{array}$

S $\quad 3.04787100 \quad-0.79448400 \quad-0.22610300$

O $\quad 3.12837400 \quad-1.10816400 \quad 1.20380800$

O $\quad 2.36907000 \quad-1.70137100 \quad-1.15810900$ 


$$
\begin{array}{lrrr}
\mathrm{C} & 4.70103800 & -0.46031700 & -0.83721700 \\
\mathrm{H} & 5.15349300 & 0.31421100 & -0.21731100 \\
\mathrm{H} & 5.26079700 & -1.39364400 & -0.75214400 \\
\mathrm{H} & 4.62902400 & -0.14373700 & -1.87746100 \\
\mathrm{O} & -1.53826000 & 2.24793500 & -1.17762900
\end{array}
$$

Electronic Energy $(\mathrm{EE})=-1295.985386$ a.u.

Electronic Energy in the solvent $\left(\mathrm{E}_{\mathrm{SP}}\right)=-1296.294952$ a.u.

Thermal Correction to Enthalpy $=0.291318$

Imaginary Freq $=0$

\section{TS-V}

$\begin{array}{lrrr}\mathrm{C} & -3.88268000 & 1.02894700 & -0.11639500 \\ \mathrm{C} & -4.95554900 & 0.13137600 & -0.03386100 \\ \mathrm{C} & -4.73838700 & -1.25445200 & -0.05997900 \\ \mathrm{C} & -3.44931100 & -1.76009500 & -0.17683300 \\ \mathrm{C} & -2.36081100 & -0.86501500 & -0.26348400 \\ \mathrm{C} & -2.58515500 & 0.54005500 & -0.21053900 \\ \mathrm{H} & -4.04072300 & 2.10197900 & -0.08984100 \\ \mathrm{H} & -5.96719600 & 0.51345300 & 0.05743900 \\ \mathrm{H} & -5.58152800 & -1.93428400 & -0.00097700 \\ \mathrm{H} & -3.27862200 & -2.83113000 & -0.23006300 \\ \mathrm{C} & -1.03884600 & -1.33206500 & -0.54666500 \\ \mathrm{O} & -0.07114600 & -0.50514600 & -0.86205500 \\ \mathrm{H} & -0.84564500 & -2.36365900 & -0.83401200 \\ \mathrm{C} & -1.40788600 & 1.44885000 & -0.22037000 \\ \mathrm{C} & -0.06465500 & 0.72637400 & -0.09187700 \\ \mathrm{C} & 1.20156300 & 1.49983100 & -0.46797300 \\ \mathrm{H} & 1.06089100 & 2.55020800 & -0.19595700 \\ \mathrm{H} & 1.43341100 & 1.42927200 & -1.53070800 \\ \mathrm{~N} & 2.26872600 & 0.91949800 & 0.35711200 \\ \mathrm{C} & 1.67405600 & 0.66096000 & 1.66883400\end{array}$




$\begin{array}{lrrr}\mathrm{H} & 2.21668500 & -0.11518800 & 2.20778300 \\ \mathrm{H} & 1.70670100 & 1.58309500 & 2.25714400 \\ \mathrm{C} & 0.15551900 & 0.28530600 & 1.43286600 \\ \mathrm{C} & -0.15142600 & -1.15043000 & 1.62972000 \\ \mathrm{H} & -0.48211000 & 0.90315800 & 2.06973200 \\ \mathrm{H} & -0.89973700 & -1.45821300 & 2.35324000 \\ \mathrm{H} & 0.58598000 & -1.88606200 & 1.31577300 \\ \mathrm{~S} & 3.13365100 & -0.41024800 & -0.36088000 \\ \mathrm{O} & 2.74198500 & -1.64844600 & 0.33463900 \\ \mathrm{O} & 2.97608100 & -0.27667100 & -1.80781100 \\ \mathrm{C} & 4.82480400 & -0.01784900 & 0.09173400 \\ \mathrm{H} & 4.90605500 & 0.01821700 & 1.17851200 \\ \mathrm{H} & 5.44669800 & -0.82024500 & -0.30973200 \\ \mathrm{H} & 5.08605500 & 0.93949900 & -0.35832600 \\ \mathrm{O} & -1.48364800 & 2.66109400 & -0.21812300\end{array}$

Electronic Energy $(\mathrm{EE})=-1295.984092$ a.u.

Electronic Energy in the solvent $\left(E_{S P}\right)=-1296.292607$ a.u.

Thermal Correction to Enthalpy $=0.29085$

Imaginary Freq = 1

\section{INT-VII}

$\begin{array}{llll}\mathrm{C} & -3.89353900 & 1.13531900 & -0.04586500 \\ \mathrm{C} & -5.00816500 & 0.30690200 & -0.07593100 \\ \mathrm{C} & -4.84461600 & -1.07970100 & -0.17918900 \\ \mathrm{C} & -3.57024000 & -1.64839400 & -0.26047400 \\ \mathrm{C} & -2.43977000 & -0.83566400 & -0.22569300 \\ \mathrm{C} & -2.61133500 & 0.56549100 & -0.13323600 \\ \mathrm{H} & -3.99264500 & 2.21297900 & 0.02805500 \\ \mathrm{H} & -6.00319900 & 0.73670400 & -0.03280200 \\ \mathrm{H} & -5.71759500 & -1.72419100 & -0.20551800 \\ \mathrm{H} & -3.46143100 & -2.72482600 & -0.35121900\end{array}$




$\begin{array}{lrrr}\mathrm{C} & -1.03466200 & -1.41531200 & -0.29608600 \\ \mathrm{O} & -0.24183800 & -0.42052000 & -0.98745700 \\ \mathrm{H} & -1.00667400 & -2.33031300 & -0.88871100 \\ \mathrm{C} & -1.42728400 & 1.44365800 & -0.08458700 \\ \mathrm{C} & -0.07864100 & 0.60326400 & -0.07305900 \\ \mathrm{C} & 1.18204400 & 1.45157000 & -0.31610800 \\ \mathrm{H} & 0.98301500 & 2.45632300 & 0.08803200 \\ \mathrm{H} & 1.47465700 & 1.51876000 & -1.36377300 \\ \mathrm{~N} & 2.17727600 & 0.76683800 & 0.47932300 \\ \mathrm{C} & 1.55183200 & 0.24708800 & 1.72119300 \\ \mathrm{H} & 2.09150700 & -0.63273900 & 2.07190100 \\ \mathrm{H} & 1.62327100 & 1.02856700 & 2.48522100 \\ \mathrm{C} & 0.08082000 & -0.05645000 & 1.35327500 \\ \mathrm{C} & -0.31104800 & -1.52389000 & 1.06636000 \\ \mathrm{H} & -0.59254600 & 0.39941900 & 2.08206400 \\ \mathrm{H} & -0.94000200 & -1.95578600 & 1.84647000 \\ \mathrm{H} & 0.57591600 & -2.14875300 & 0.93506400 \\ \mathrm{~S} & 3.30040200 & -0.30329400 & -0.38893200 \\ \mathrm{O} & 3.00924500 & -1.66174000 & 0.07452900 \\ \mathrm{O} & 3.19591400 & 0.08083100 & -1.79114700 \\ \mathrm{C} & 4.87670200 & 0.23122800 & 0.27750500 \\ \mathrm{H} & 4.87457700 & 0.08812600 & 1.35865800 \\ \mathrm{H} & 5.63425700 & -0.40393300 & -0.18764300 \\ \mathrm{H} & 5.02978600 & 1.27604400 & 0.00713100 \\ \mathrm{O} & -1.39492600 & 2.64365400 & 0.03233500\end{array}$

Electronic Energy (EE) = -1296.020743 a.u.

Electronic Energy in the solvent $\left(E_{S P}\right)=-1296.322518$ a.u.

Thermal Correction to Enthalpy $=0.293421$

Imaginary Freq $=0$ 


\section{INT-V(3b)}

C

$\begin{array}{lll}1.43266000 & 3.42495400 & 0.14969100\end{array}$

$\begin{array}{lllll}\text { C } & 2.67204300 & 3.92385000 & -0.25993200\end{array}$

C $\quad 3.75441400 \quad 3.05985200 \quad-0.41786900$

C $\quad 3.60365500 \quad 1.69152700 \quad-0.18293400$

$\begin{array}{llll}\text { C } & 2.36123100 & 1.16709000 & 0.19469600\end{array}$

$\begin{array}{llll}\text { C } & 1.27510400 & 2.05442100 & 0.35749600\end{array}$

$\begin{array}{llll}\mathrm{H} & 0.59776400 & 4.09852000 & 0.31381900\end{array}$

$\mathrm{H} \quad 2.79347600 \quad 4.98910800 \quad-0.42688500$

$\mathrm{H} \quad 4.72635100 \quad 3.45206700 \quad-0.69842600$

$\mathrm{H} \quad 4.46371900 \quad 1.03662500 \quad-0.25266900$

$\begin{array}{llll}\text { C } & 2.12477900 & -0.25806800 & 0.58808000\end{array}$

O $\quad 1.11085200 \quad-0.49219800 \quad 1.25736500$

$\begin{array}{llll}\text { C } & -0.05688700 & 1.55728400 & 0.84410100\end{array}$

$\begin{array}{lllll}\text { C } & -0.97637800 & 0.74492900 & -0.02693500\end{array}$

$\begin{array}{llll}\text { C } & -2.12108600 & 0.16128400 & 0.78070900\end{array}$

$\mathrm{H} \quad-2.24500600 \quad 0.85839700 \quad 1.65028200$

$\mathrm{H} \quad-1.95620000 \quad-0.84184200 \quad 1.18537500$

$\mathrm{N} \quad \begin{array}{llll}-3.25590300 & 0.19720600 & -0.09523600\end{array}$

C $\quad-3.08868700 \quad 1.10897700 \quad-1.23483600$

$\mathrm{H} \quad-3.50259400 \quad 0.63601500 \quad-2.13301000$

$\mathrm{H} \quad-3.66240500 \quad 2.03116000 \quad-1.05794400$

C $\quad-1.59925300 \quad 1.36576200 \quad-1.30399100$

C $\quad-0.68224900 \quad 0.17773900 \quad-1.37731800$

$\mathrm{H} \quad-1.25871000 \quad 2.32476900 \quad-1.67846300$

$\mathrm{H} \quad 0.30289200 \quad 0.37590200 \quad-1.78721400$

$\mathrm{H} \quad-1.08313600 \quad-0.80445800 \quad-1.61453800$

S $\quad-4.44781900 \quad-1.13540100 \quad-0.14815200$

O $\quad-4.97691700-1.08839900-1.50324000$

O $\quad-3.74426900 \quad-2.28608200 \quad 0.39916300$

C $\quad-5.68660300 \quad-0.56156900 \quad 1.01760000$ 


$\begin{array}{lrrr}\mathrm{H} & -6.10703200 & 0.37635000 & 0.65360700 \\ \mathrm{H} & -6.45393600 & -1.33953800 & 1.04177800 \\ \mathrm{H} & -5.22663500 & -0.45277200 & 2.00038800 \\ \mathrm{O} & -0.51450000 & 1.98184000 & 1.90052000 \\ \mathrm{C} & 3.04708900 & -1.35568500 & 0.21416800 \\ \mathrm{C} & 3.13531600 & -2.45929900 & 1.08229400 \\ \mathrm{C} & 3.76484500 & -1.36979800 & -0.99559400 \\ \mathrm{C} & 3.95387600 & -3.53668000 & 0.76357100 \\ \mathrm{H} & 2.56447000 & -2.44822500 & 2.00435000 \\ \mathrm{C} & 4.56496100 & -2.46312600 & -1.32034000 \\ \mathrm{H} & 3.67093700 & -0.55014800 & -1.69991600 \\ \mathrm{C} & 4.66961300 & -3.54005900 & -0.43765600 \\ \mathrm{H} & 4.03279700 & -4.37653600 & 1.44621800 \\ \mathrm{H} & 5.10416600 & -2.47608400 & -2.26200900 \\ \mathrm{H} & 5.30353100 & -4.38492600 & -0.68813600\end{array}$

Electronic Energy $(\mathrm{EE})=-1527.050191$ a.u.

Imaginary Freq $=0$

\section{INT-VII(3b)}

$\begin{array}{lrrr}\mathrm{C} & 3.00390400 & -2.87562300 & -0.37790900 \\ \mathrm{C} & 4.28536900 & -2.46645500 & -0.03141400 \\ \mathrm{C} & 4.49370900 & -1.16034400 & 0.42479700 \\ \mathrm{C} & 3.43402000 & -0.25596900 & 0.52174200 \\ \mathrm{C} & 2.13890200 & -0.64813600 & 0.18049300 \\ \mathrm{C} & 1.93652500 & -1.96785900 & -0.28424800 \\ \mathrm{H} & 2.80556800 & -3.88157700 & -0.73269800 \\ \mathrm{H} & 5.11941900 & -3.15385400 & -0.12494800 \\ \mathrm{H} & 5.49266400 & -0.83797100 & 0.70139100 \\ \mathrm{H} & 3.61747900 & 0.75687300 & 0.86166600 \\ \mathrm{C} & 0.92773400 & 0.31891800 & 0.30373400 \\ \mathrm{O} & 0.00389300 & -0.14883300 & -0.71385600\end{array}$




\begin{tabular}{|c|c|c|c|}
\hline $\mathrm{C}$ & 0.57449500 & -2.44804100 & -0.60613000 \\
\hline $\mathrm{C}$ & -0.49927900 & -1.36300300 & -0.25930200 \\
\hline $\mathrm{C}$ & -1.90713000 & -1.66349200 & -0.80648700 \\
\hline $\mathrm{H}$ & -2.01750100 & -2.75726400 & -0.84030800 \\
\hline $\mathrm{H}$ & -2.09298700 & -1.23928300 & -1.79262600 \\
\hline $\mathrm{N}$ & -2.78465400 & -1.09601000 & 0.19970500 \\
\hline $\mathrm{C}$ & -2.16844600 & -1.28235200 & 1.53440700 \\
\hline $\mathrm{H}$ & -2.51211400 & -0.50915500 & 2.22161800 \\
\hline $\mathrm{H}$ & -2.48833800 & -2.25704700 & 1.91819300 \\
\hline $\mathrm{C}$ & -0.63777700 & -1.25069600 & 1.30390600 \\
\hline $\mathrm{C}$ & 0.10615000 & 0.06935200 & 1.60187700 \\
\hline $\mathrm{H}$ & -0.16374500 & -2.09454000 & 1.81001700 \\
\hline $\mathrm{H}$ & 0.73359100 & 0.00290200 & 2.49192800 \\
\hline $\mathrm{H}$ & -0.59895700 & 0.89606900 & 1.71748100 \\
\hline S & -3.49659100 & 0.47624700 & -0.15641000 \\
\hline $\mathrm{O}$ & -2.96805400 & 1.41807900 & 0.83501700 \\
\hline $\mathrm{O}$ & -3.31664000 & 0.68084000 & -1.59026300 \\
\hline $\mathrm{C}$ & -5.22780200 & 0.16641500 & 0.19597200 \\
\hline $\mathrm{H}$ & -5.33005100 & -0.13731800 & 1.23833000 \\
\hline $\mathrm{H}$ & -5.74976600 & 1.11005200 & 0.02233300 \\
\hline $\mathrm{H}$ & -5.58712800 & -0.60497700 & -0.48506800 \\
\hline $\mathrm{O}$ & 0.25991700 & -3.54791100 & -0.99473200 \\
\hline $\mathrm{C}$ & 1.30842000 & 1.75102900 & 0.02207600 \\
\hline $\mathrm{C}$ & 1.63610500 & 2.62000600 & 1.07811200 \\
\hline $\mathrm{C}$ & 1.36870800 & 2.21796800 & -1.30264400 \\
\hline $\mathrm{C}$ & 2.00261000 & 3.93661700 & 0.81433200 \\
\hline $\mathrm{H}$ & 1.59462100 & 2.27428400 & 2.10621700 \\
\hline $\mathrm{C}$ & 1.72875900 & 3.53538100 & -1.56102000 \\
\hline $\mathrm{H}$ & 1.11700800 & 1.55182600 & -2.11993300 \\
\hline $\mathrm{C}$ & 2.04904900 & 4.39831900 & -0.50519100 \\
\hline $\mathrm{H}$ & 2.24520800 & 4.60424600 & 1.63443800 \\
\hline
\end{tabular}




$$
\begin{array}{llll}
\mathrm{H} & 1.76180100 & 3.89421700 & -2.58444400 \\
\mathrm{H} & 2.33417800 & 5.42502100 & -0.71091400
\end{array}
$$

Electronic Energy $(\mathrm{EE})=-1527.082838$ a.u.

Imaginary Freq $=0$

3b

$\mathrm{C}$

$\begin{array}{lll}-1.98997100 & 3.62160600 & 0.09535500\end{array}$

C $\quad-3.34701800 \quad 3.92811700 \quad 0.07268600$

C $\quad-4.28794600 \quad 2.92004400 \quad-0.14016900$

$\begin{array}{llll}\text { C } & -3.85880500 & 1.60636200 & -0.31628700\end{array}$

C $\quad-2.49623000 \quad 1.27579600 \quad-0.30706200$

C $\quad-1.53749100 \quad 2.30395800 \quad-0.10692600$

$\mathrm{H} \quad-1.25505200 \quad 4.40098500 \quad 0.26618400$

$\mathrm{H} \quad-3.66835600 \quad 4.95467400 \quad 0.22099200$

$\mathrm{H} \quad-5.34770500 \quad 3.15317500 \quad-0.16807000$

$\mathrm{H} \quad-4.58859200 \quad 0.82180400 \quad-0.48621400$

C $\quad-2.07176700 \quad-0.13334900 \quad-0.60432900$

$\begin{array}{llll}\text { O } & -1.10439800 & -0.35247400 & -1.32557100\end{array}$

$\begin{array}{llll}\text { C } & -0.13135800 & 2.06104800 & -0.06570800\end{array}$

$\begin{array}{llll}\text { C } & 1.07277200 & 1.94164400 & 0.00301900\end{array}$

$\begin{array}{llll}\text { C } & 2.52361800 & 1.77019700 & 0.02577200\end{array}$

$\begin{array}{llll}\mathrm{H} & 2.94364800 & 2.16690600 & 0.95288500\end{array}$

$\begin{array}{llll}\mathrm{H} & 2.96843200 & 2.35430100 & -0.79677900\end{array}$

$\begin{array}{llll}\mathrm{N} & 2.89724400 & 0.33967100 & -0.07058800\end{array}$

C $\quad 2.56793500 \quad-0.26523400 \quad-1.39474100$

$\begin{array}{lllll}\mathrm{H} & 1.53414900 & 0.03170300 & -1.58728800\end{array}$

$\begin{array}{llll}\mathrm{H} & 3.21021800 & 0.17487700 & -2.17152300\end{array}$

C $\quad 2.66209000 \quad-1.76925900-1.44811400$

C $\quad \begin{array}{llll}1.59158200 & -2.56507700 & -1.44002600\end{array}$

$\mathrm{H} \quad 3.66153100 \quad-2.18913600 \quad-1.53530900$

$\mathrm{H} \quad 1.69269500 \quad-3.64465500 \quad-1.51514800$ 


$\begin{array}{lrrr}\mathrm{H} & 0.58534000 & -2.15986400 & -1.36851500 \\ \mathrm{~S} & 4.43162700 & -0.03354500 & 0.57507700 \\ \mathrm{O} & 5.32694200 & -0.50812400 & -0.48921700 \\ \mathrm{O} & 4.83459800 & 1.08555700 & 1.43712000 \\ \mathrm{C} & 4.04627600 & -1.43082400 & 1.64375300 \\ \mathrm{H} & 4.99280100 & -1.78403400 & 2.05711300 \\ \mathrm{H} & 3.38293300 & -1.07941900 & 2.43367100 \\ \mathrm{H} & 3.56442500 & -2.20643900 & 1.04913000 \\ \mathrm{C} & -2.85868200 & -1.27632100 & -0.03548800 \\ \mathrm{C} & -2.81369900 & -2.50853800 & -0.70749700 \\ \mathrm{C} & -3.57404000 & -1.17834100 & 1.16787700 \\ \mathrm{C} & -3.48731900 & -3.61399200 & -0.19823800 \\ \mathrm{H} & -2.24918500 & -2.57451000 & -1.63148000 \\ \mathrm{C} & -4.23348100 & -2.29274400 & 1.68632800 \\ \mathrm{H} & -3.59661800 & -0.23772700 & 1.70769700 \\ \mathrm{C} & -4.19713400 & -3.50827700 & 1.00168200 \\ \mathrm{H} & -3.45883300 & -4.55933800 & -0.73181000 \\ \mathrm{H} & -4.77351600 & -2.21196300 & 2.62488300 \\ \mathrm{H} & -4.71817300 & -4.37288900 & 1.40247700\end{array}$

Electronic Energy $(\mathrm{EE})=-1452.055196$ a.u.

$\mathrm{EE}+$ Thermal Free Energy Correction $=-1451.764363$ a.u.

Imaginary Freq $=0$
$4 \mathbf{b}$
C $\quad 2.07442200 \quad 3.35394900 \quad-0.12515200$
C $\quad 3.39449900 \quad 3.47335700 \quad-0.56035300$
C $\quad 4.22244100 \quad 2.35182900 \quad-0.58182400$
C $\quad 3.72392700 \quad 1.11335900 \quad-0.17858000$
$\begin{array}{llll}\text { C } & 2.39223400 & 0.97490300 & 0.23928800\end{array}$
$\begin{array}{llll}\text { C } & 1.55843800 & 2.11578100 & 0.26682400\end{array}$
$\begin{array}{llll}\mathrm{H} & 1.43036200 & 4.22618200 & -0.07622700\end{array}$ 


\begin{tabular}{|c|c|c|c|}
\hline $\mathrm{H}$ & 3.77784200 & 4.44216600 & -0.86625100 \\
\hline $\mathrm{H}$ & 5.25791800 & 2.44083800 & -0.89578200 \\
\hline $\mathrm{H}$ & 4.38243500 & 0.25208100 & -0.15413500 \\
\hline $\mathrm{C}$ & 1.88286600 & -0.31356100 & 0.81696000 \\
\hline $\mathrm{O}$ & 1.05014900 & -0.27362900 & 1.71882400 \\
\hline $\mathrm{C}$ & 0.12776000 & 2.09142500 & 0.75295700 \\
\hline $\mathrm{C}$ & -0.88772200 & 1.21261800 & 0.07760500 \\
\hline $\mathrm{C}$ & -2.03788200 & 0.71501900 & 0.95154600 \\
\hline $\mathrm{H}$ & -2.22736500 & 1.45047000 & 1.73838500 \\
\hline $\mathrm{H}$ & -1.81462800 & -0.25412200 & 1.40220000 \\
\hline $\mathrm{N}$ & -3.21650900 & 0.61804000 & 0.05480300 \\
\hline $\mathrm{C}$ & -3.02485100 & 1.51553800 & -1.10785400 \\
\hline $\mathrm{H}$ & -3.51393400 & 1.09606100 & -1.98864900 \\
\hline $\mathrm{H}$ & -3.48177500 & 2.48899600 & -0.89067400 \\
\hline $\mathrm{C}$ & -1.51751600 & 1.67059500 & -1.24091100 \\
\hline $\mathrm{C}$ & -0.65565300 & 0.44146700 & -1.19784800 \\
\hline $\mathrm{H}$ & -1.14281500 & 2.55504800 & -1.74819100 \\
\hline $\mathrm{H}$ & 0.31905900 & 0.52220900 & -1.66753800 \\
\hline $\mathrm{H}$ & -1.11283300 & -0.54146500 & -1.27301500 \\
\hline $\mathrm{S}$ & -3.88676800 & -0.91177000 & -0.22094000 \\
\hline $\mathrm{O}$ & -4.65625000 & -0.82585500 & -1.46761600 \\
\hline $\mathrm{O}$ & -2.88319000 & -1.97220100 & -0.04355400 \\
\hline $\mathrm{C}$ & -5.04405100 & -1.04714000 & 1.15156600 \\
\hline $\mathrm{H}$ & -5.78504600 & -0.25355200 & 1.06011000 \\
\hline $\mathrm{H}$ & -5.51295000 & -2.02990400 & 1.07783600 \\
\hline $\mathrm{H}$ & -4.49283000 & -0.96168200 & 2.08890000 \\
\hline $\mathrm{O}$ & -0.21736600 & 2.87639700 & 1.62226600 \\
\hline $\mathrm{C}$ & 2.38970800 & -1.63265600 & 0.32388400 \\
\hline $\mathrm{C}$ & 2.28971100 & -2.73365900 & 1.19021300 \\
\hline $\mathrm{C}$ & 2.88203500 & -1.82771500 & -0.97636400 \\
\hline $\mathrm{C}$ & 2.70124700 & -3.99577100 & 0.77603700 \\
\hline
\end{tabular}




$$
\begin{array}{lrrr}
\mathrm{H} & 1.88273000 & -2.57373600 & 2.18269800 \\
\mathrm{C} & 3.27737900 & -3.09783600 & -1.39528300 \\
\mathrm{H} & 2.93395300 & -0.99447200 & -1.66885600 \\
\mathrm{C} & 3.19629200 & -4.18021500 & -0.51839200 \\
\mathrm{H} & 2.63054000 & -4.83885700 & 1.45658500 \\
\mathrm{H} & 3.64405500 & -3.24222100 & -2.40702400 \\
\mathrm{H} & 3.51068200 & -5.16740500 & -0.84422000
\end{array}
$$

Electronic Energy $(\mathrm{EE})=-1527.335568$ a.u.

$\mathrm{EE}+$ Thermal Free Energy Correction $=-1527.036026$ a.u.

Imaginary Freq $=0$

$2 \mathbf{b}^{\prime}$

$\mathrm{C}$

$3.09302400-2.78453100-0.41643400$

C $\quad 4.36474600-2.35005000-0.06039400$

C $\quad 4.53486400 \quad-1.05290700 \quad 0.43160800$

C $\quad 3.44450100 \quad-0.19008900 \quad 0.55755400$

C $\quad 2.16204800 \quad-0.60893500 \quad 0.19593400$

C $\quad 1.99335200-1.92727000 \quad-0.28464800$

$\mathrm{H} \quad 2.91706600 \quad-3.78828300 \quad-0.78975100$

$\mathrm{H} \quad 5.21858500 \quad-3.01302500 \quad-0.16131400$

$\mathrm{H} \quad \begin{array}{llll}5.52567900 & -0.70427700 & 0.70864900\end{array}$

$\begin{array}{llll}\mathrm{H} & 3.59851500 & 0.82320000 & 0.91308300\end{array}$

$\begin{array}{llll}\text { C } & 0.92350600 & 0.30245400 & 0.29369100\end{array}$

O $\quad-0.01401800-0.14792800 \quad-0.70287300$

C $\quad 0.62644900-2.43834800 \quad-0.58510900$

C $\quad-0.46433900-1.42849200 \quad-0.25048400$

C $\quad-1.86135100 \quad-1.71385800 \quad-0.78850100$

$\mathrm{H} \quad-2.02099000 \quad-2.79558000 \quad-0.83329300$

$\mathrm{H} \quad-2.02401500-1.28255300 \quad-1.77534400$

$\mathrm{N} \quad \quad-2.78855300 \quad-1.12898400 \quad 0.20419400$

C $\quad-2.15548800 \quad-1.29184200 \quad 1.53267900$ 


\begin{tabular}{|c|c|c|c|}
\hline $\mathrm{H}$ & -2.48986000 & -0.50964400 & 2.21495900 \\
\hline $\mathrm{H}$ & -2.46932000 & -2.26152000 & 1.93481500 \\
\hline $\mathrm{C}$ & -0.62134800 & -1.28000300 & 1.30603200 \\
\hline $\mathrm{C}$ & 0.12374000 & 0.03917000 & 1.60374400 \\
\hline $\mathrm{H}$ & -0.16292500 & -2.12019300 & 1.83477600 \\
\hline $\mathrm{H}$ & 0.75990500 & -0.02873900 & 2.48936500 \\
\hline $\mathrm{H}$ & -0.58712700 & 0.85914800 & 1.73317400 \\
\hline$S$ & -3.46409900 & 0.39758100 & -0.15323000 \\
\hline $\mathrm{O}$ & -3.01858500 & 1.39345500 & 0.83320500 \\
\hline $\mathrm{O}$ & -3.31486200 & 0.63314300 & -1.59140900 \\
\hline $\mathrm{C}$ & -5.20913400 & 0.08743200 & 0.17208100 \\
\hline $\mathrm{H}$ & -5.32776500 & -0.21774900 & 1.21230300 \\
\hline $\mathrm{H}$ & -5.73878200 & 1.02395600 & -0.01126900 \\
\hline $\mathrm{H}$ & -5.55490700 & -0.69271400 & -0.50553400 \\
\hline $\mathrm{O}$ & 0.39539400 & -3.56575100 & -0.99373600 \\
\hline $\mathrm{C}$ & 1.24726700 & 1.76110700 & 0.02295900 \\
\hline $\mathrm{C}$ & 1.55426500 & 2.64157400 & 1.06726100 \\
\hline $\mathrm{C}$ & 1.27314200 & 2.23547500 & -1.29469300 \\
\hline $\mathrm{C}$ & 1.88500500 & 3.97161900 & 0.80067700 \\
\hline $\mathrm{H}$ & 1.53043200 & 2.29525100 & 2.09629500 \\
\hline $\mathrm{C}$ & 1.59507200 & 3.56553400 & -1.55896200 \\
\hline $\mathrm{H}$ & 1.02164000 & 1.56063900 & -2.10477500 \\
\hline $\mathrm{C}$ & 1.90583300 & 4.43727600 & -0.51349600 \\
\hline $\mathrm{H}$ & 2.11763800 & 4.64288800 & 1.62222500 \\
\hline $\mathrm{H}$ & 1.60029200 & 3.92239600 & -2.58475000 \\
\hline $\mathrm{H}$ & 2.15701800 & 5.47313300 & -0.72178700 \\
\hline
\end{tabular}




\begin{tabular}{|c|c|c|c|}
\hline $\mathrm{C}$ & -3.26682200 & -1.44169000 & 0.42405700 \\
\hline $\mathrm{C}$ & -4.51404700 & -1.57483800 & -0.19241900 \\
\hline $\mathrm{C}$ & -5.10197800 & -0.48610100 & -0.83749600 \\
\hline $\mathrm{C}$ & -4.43691200 & 0.73716600 & -0.85477700 \\
\hline $\mathrm{C}$ & -3.18339100 & 0.88000000 & -0.24479200 \\
\hline $\mathrm{C}$ & -2.58070900 & -0.22600300 & 0.39301900 \\
\hline $\mathrm{H}$ & -2.82529500 & -2.28878800 & 0.94040900 \\
\hline $\mathrm{H}$ & -5.02834300 & -2.53078300 & -0.16142900 \\
\hline $\mathrm{H}$ & -6.07208000 & -0.58878500 & -1.31319100 \\
\hline $\mathrm{H}$ & -4.89034500 & 1.60009600 & -1.33646500 \\
\hline $\mathrm{C}$ & -2.53802100 & 2.21048300 & -0.25000500 \\
\hline $\mathrm{O}$ & -1.48353700 & 2.47051000 & 0.29862800 \\
\hline $\mathrm{H}$ & -3.10048100 & 2.99539300 & -0.80031700 \\
\hline $\mathrm{C}$ & -1.24869900 & -0.13349700 & 1.11544400 \\
\hline $\mathrm{C}$ & 0.01540500 & -0.19201500 & 0.32407500 \\
\hline $\mathrm{C}$ & 1.30358700 & 0.15369200 & 1.06613800 \\
\hline $\mathrm{H}$ & 1.19998300 & -0.12818200 & 2.11753000 \\
\hline $\mathrm{H}$ & 1.53711400 & 1.21865200 & 1.00084300 \\
\hline $\mathrm{N}$ & 2.36932000 & -0.65484900 & 0.42261500 \\
\hline $\mathrm{C}$ & 1.76487100 & -1.82631500 & -0.25121600 \\
\hline $\mathrm{H}$ & 2.34931000 & -2.09198800 & -1.13377300 \\
\hline $\mathrm{H}$ & 1.77199100 & -2.68180100 & 0.43581700 \\
\hline $\mathrm{C}$ & 0.33144300 & -1.41593300 & -0.55294000 \\
\hline $\mathrm{C}$ & 0.07937600 & -0.08309400 & -1.18218300 \\
\hline $\mathrm{H}$ & -0.40635100 & -2.20307400 & -0.67715300 \\
\hline $\mathrm{H}$ & -0.85745100 & 0.03536600 & -1.71550800 \\
\hline $\mathrm{H}$ & 0.90914300 & 0.48719400 & -1.59060500 \\
\hline $\mathrm{S}$ & 3.62265200 & 0.16253900 & -0.37110800 \\
\hline $\mathrm{O}$ & 4.23696900 & -0.79444000 & -1.29837500 \\
\hline $\mathrm{O}$ & 3.17663700 & 1.47822100 & -0.85338700 \\
\hline
\end{tabular}




$\begin{array}{llll}\mathrm{C} & 4.76996600 & 0.45763500 & 0.98431800 \\ \mathrm{H} & 5.09343900 & -0.50469500 & 1.37981600 \\ \mathrm{H} & 5.61338800 & 1.01464900 & 0.57264000 \\ \mathrm{H} & 4.26713100 & 1.04788200 & 1.75148300 \\ \mathrm{O} & -1.23922400 & -0.12363400 & 2.33588300\end{array}$

Electronic Energy $(\mathrm{EE})=-1296.269361$ a.u.

Imaginary Freq $=0$ 


\section{X-Ray Crystallographic Analysis}

\section{CCDC: 2042834}

Table 1: Crystal data and structure refinement for $\mathbf{2 m}$

Identification code

Empirical formula

Formula weight

Temperature

Wavelength

Crystal system

Space group

Z

Unit cell dimensions

\section{Volume}

Density (calculated)

Absorption coefficient

Crystal shape

Crystal size

Crystal colour

Theta range for data collection

Index ranges

Reflections collected

Independent reflections

Observed reflections

Absorption correction

Max. and min. transmission

Refinement method

Data/restraints/parameters

Goodness-of-fit on $\mathrm{F}^{2}$

Final R indices (I>2sigma(I))

Largest diff. peak and hole

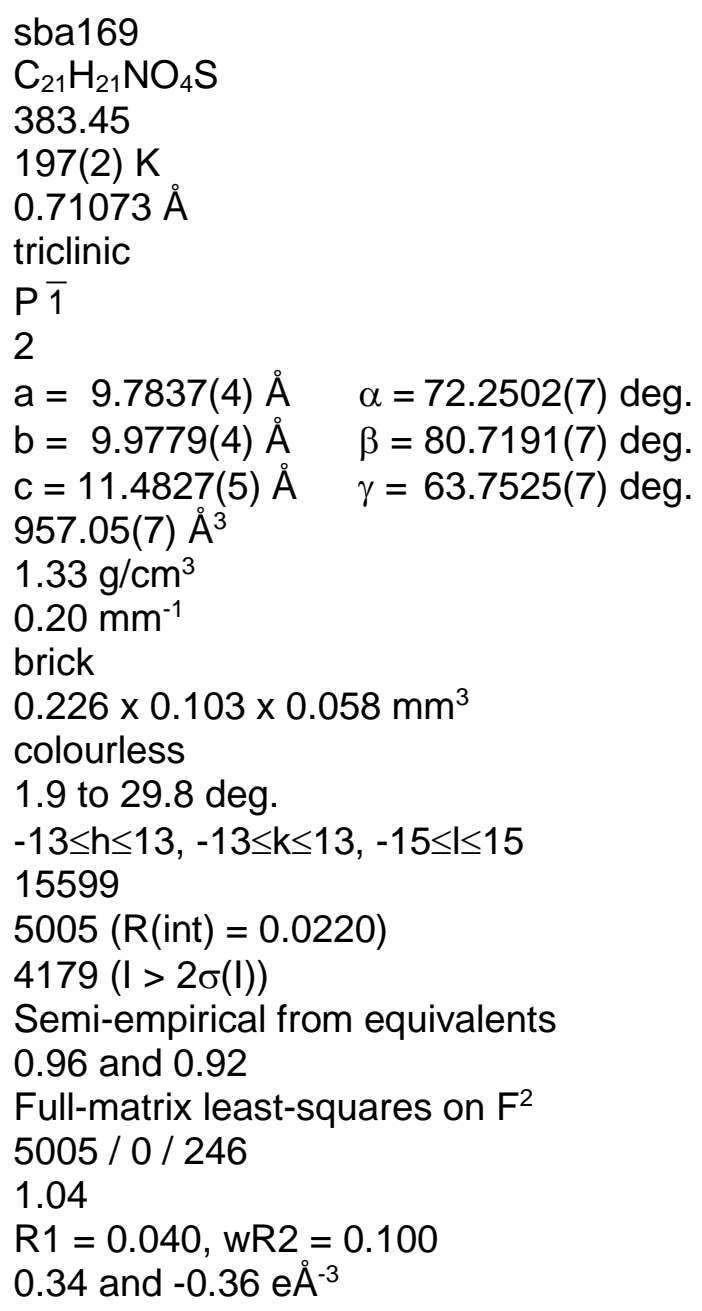

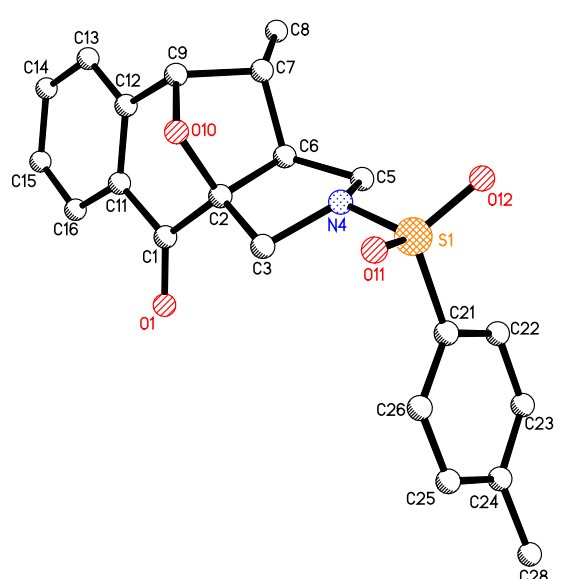

Thermal ellipsoid plot for compound (2m), displacement ellipsoids are drawn at the $50 \%$ probability level.

Dichloromethane was used as solvent at room emperature for crystal prepration. 
Vorschlag für eine stichwortartige Experimentbeschreibung (suggestion for a short experimental part):

sba169: colourless crystal (brick), dimensions $0.226 \times 0.103 \times 0.058 \mathrm{~mm}^{3}$, crystal system triclinic, space group $P \overline{1}, \quad Z=2, \quad a=9.7837(4) \AA ⿻ \quad b=9.9779(4) \quad \AA, \quad c=11.4827(5) \AA$, alpha=72.2502(7) deg, beta=80.7191(7) deg, gamma=63.7525(7) deg, $V=957.05(7) \AA^{3}$, rho=1.331 g/ $\mathrm{cm}^{3}, T=197(2) \mathrm{K}$, Theta $\max =29.781 \mathrm{deg}$, radiation MoK $\alpha$, lambda=0.71073 $\AA$, 0.5 deg omega-scans with CCD area detector, covering the asymmetric unit in reciprocal space with a mean redundancy of 2.85 and a completeness of $91.5 \%$ to a resolution of $0.72 \AA, 15599$ reflections measured, 5005 unique $(R($ int $)=0.0220), 4179$ observed $(I>2 \sigma(I))$, intensities were corrected for Lorentz and polarization effects, an empirical scaling and absorption correction was applied using SADABS ${ }^{[1]}$ based on the Laue symmetry of the reciprocal space, $\mathrm{mu}=0.20 \mathrm{~mm}^{-1}, \mathrm{~T}_{\min }=0.92, \mathrm{~T}_{\max }=0.96$, structure solved with SHELXT-2018/2 (Sheldrick 2015) ${ }^{[2]}$ and refined against $F^{2}$ with a Full-matrix least-squares algorithm using the SHELXL-2018/3 (Sheldrick, 2018) software ${ }^{[3]}, 246$ parameters refined, hydrogen atoms were treated using appropriate riding models, goodness of fit 1.04 for observed reflections, final residual values $\mathrm{R} 1(\mathrm{~F})=0.040, \mathrm{wR}\left(\mathrm{F}^{2}\right)=0.100$ for observed reflections, residual electron density -0.36 to 0.34 $\mathrm{e}^{-3}$. CCDC 2042834 contains the supplementary crystallographic data for this paper. The data can be obtained free of charge from The Cambridge Crystallographic Data Centre via www.ccdc.cam.ac.uk/structures.

Lit. 1: (SADABS-2016/2 - Bruker AXS area detector scaling and absorption correction) Krause, L., Herbst-Irmer, R., Sheldrick G.M. \& Stalke D., J. Appl. Cryst. 48 (2015) 3-10.

Lit. 2: (SHELXT - Integrated space-group and crystal structure determination) Sheldrick G. M., Acta Cryst. A71 (2015) 3-8.

Lit. 3: (program SHELXL-2018/3 (Sheldrick, 2018) for structure refinement) Sheldrick G. M., Acta Cryst. (2015). C71, 3-8

Lit. APEX, APEX2, SMART, SAINT, SAINT-Plus:

Bruker (2007). "Program name(s)". Bruker AXS Inc., Madison, Wisconsin, USA. 
12. ${ }^{1} \mathrm{H},{ }^{13} \mathrm{C}$ NMR, and HRMS spectra

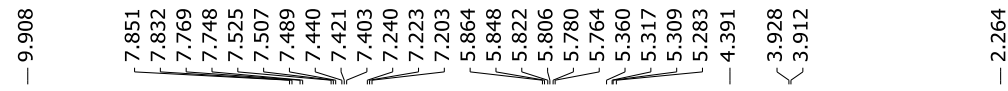
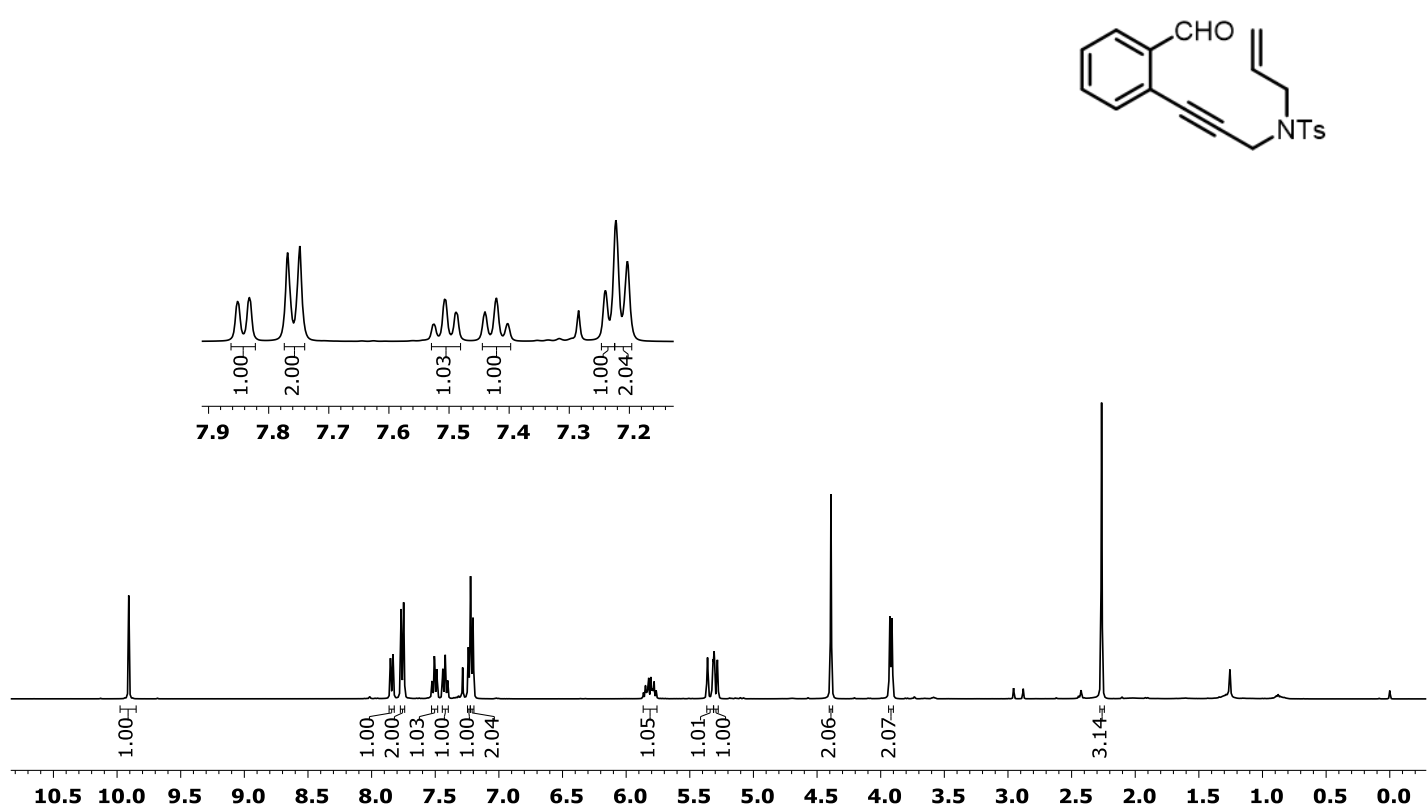

${ }^{1} \mathrm{H}$ NMR Compound $1 \mathbf{a}\left(400 \mathrm{MHz}, \mathrm{CDCl}_{3}\right)$

\begin{tabular}{|c|c|c|c|}
\hline & 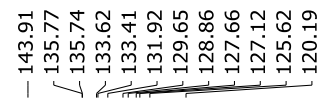 & $\begin{array}{l}\dot{\infty} \\
\text { । }\end{array}$ & \\
\hline
\end{tabular}
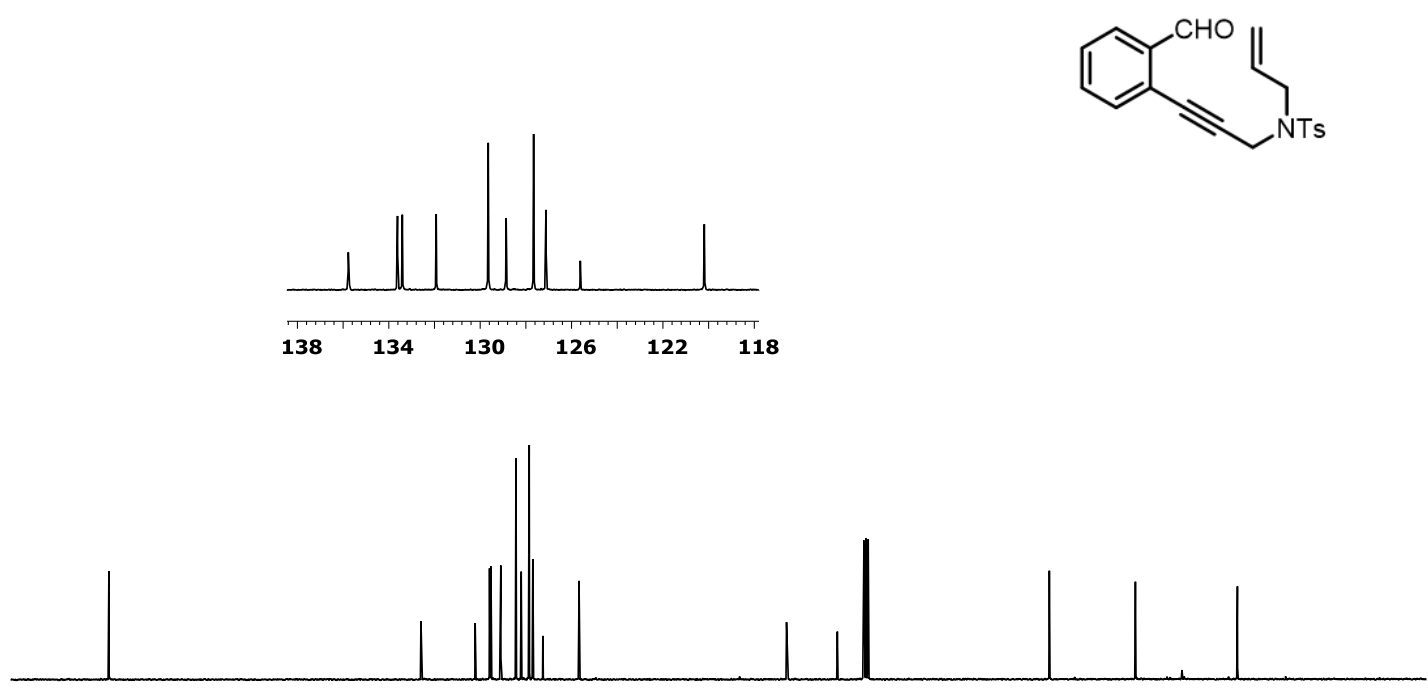

$\begin{array}{lllllllllllllllllllll}200 & 190 & 180 & 170 & 160 & 150 & 140 & 130 & 120 & 110 & 100 & 90 & 80 & 70 & 60 & 50 & 40 & 30 & 20 & 10 & 0\end{array}$

${ }^{13} \mathrm{C}$ NMR Compound $1 \mathrm{a}\left(100 \mathrm{MHz}, \mathrm{CDCl}_{3}\right)$ 


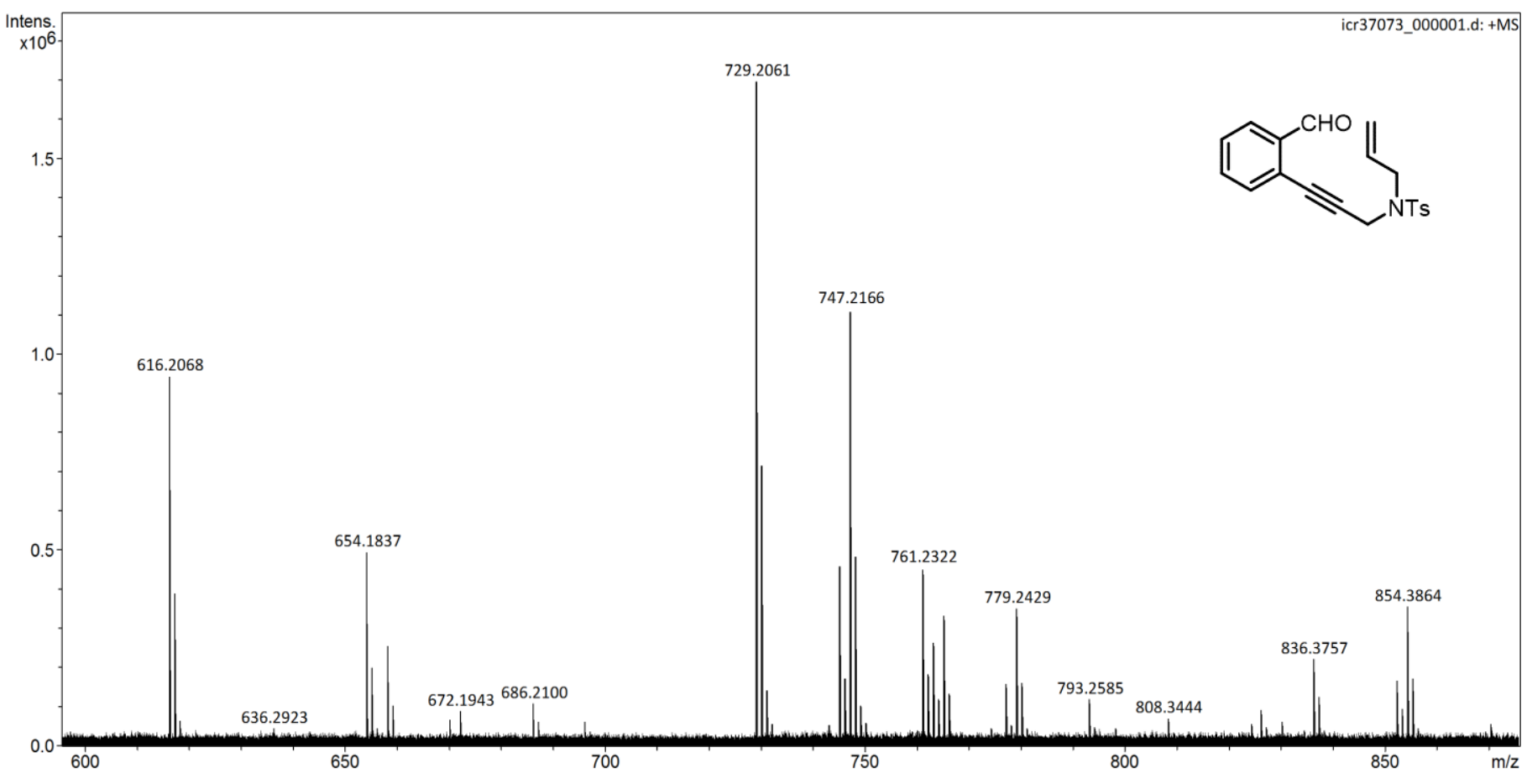

HRMS-ESI Compound 1a

$\begin{array}{rcrrrrrr}\text { Meas. } \mathrm{m} / \mathrm{z} & \text { Ion Formula } & \mathrm{m} / \mathrm{z} & \mathrm{err}[\mathrm{ppm}] & \mathrm{mSigma} & \mathrm{rdb} & \mathrm{e}^{-} \text {Conf } & \text { N-Rule } \\ 729.2061 & \mathrm{C} 40 \mathrm{H} 30 \mathrm{~N} 6 \mathrm{NaO} 7 & 729.2068 & 1.0 & 14.7 & 28.5 & \text { even } & \text { ok } \\ & \mathrm{C} 40 \mathrm{H} 35 \mathrm{~N} 5 \mathrm{O} 5 \mathrm{~S} 2 & 729.2074 & 1.8 & 17.8 & 26.0 & \text { odd } & \text { ok } \\ & \mathrm{C} 40 \mathrm{H} 38 \mathrm{~N} 2 \mathrm{NaO} 6 \mathrm{~S} 2 & 729.2063 & 0.4 & 19.1 & 22.5 & \text { even } & \text { ok }\end{array}$



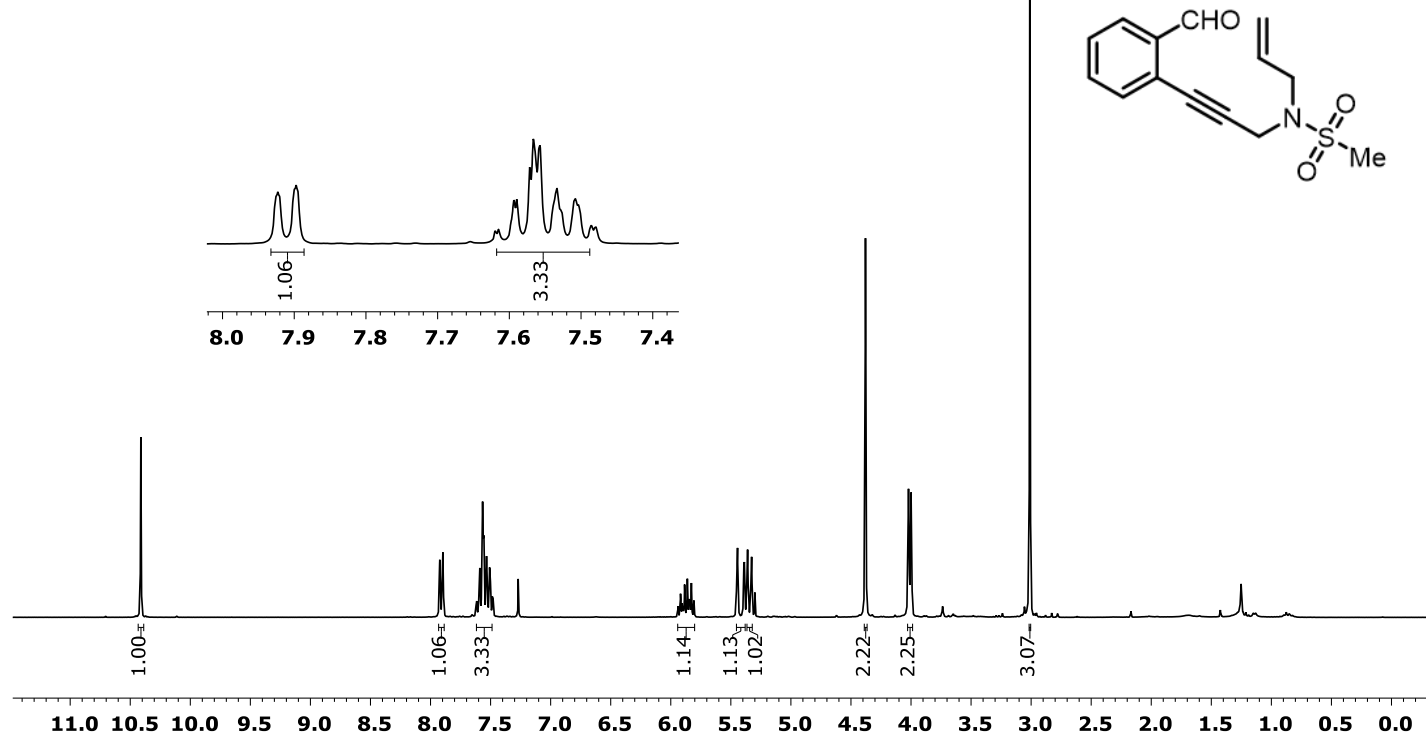

${ }^{1} \mathrm{H}$ NMR Compound $\mathbf{1 b}\left(300 \mathrm{MHz}, \mathrm{CDCl}_{3}\right)$

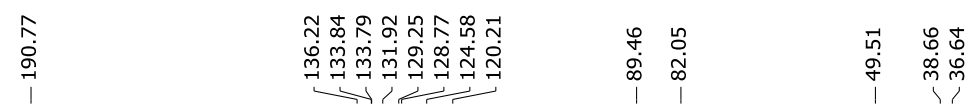
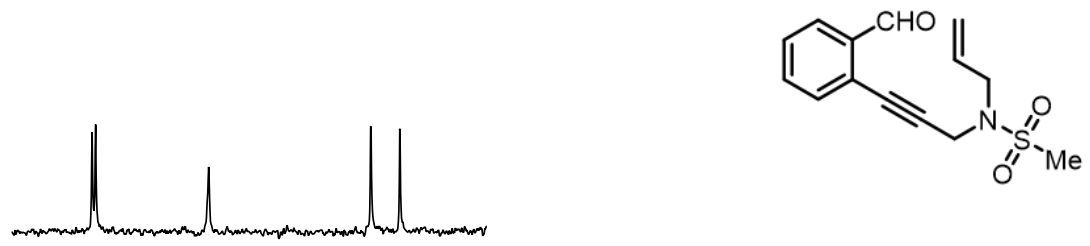

$\begin{array}{llllllll}135 & 134 & 133 & 132 & 131 & 130 & 129 & 128\end{array}$

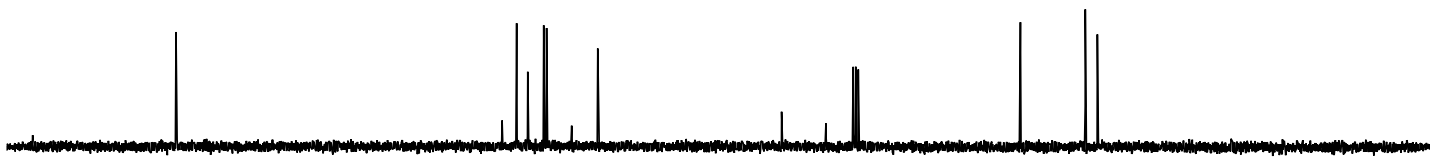

$\begin{array}{lllllllllllllllllllllllllllll}210 & 200 & 190 & 180 & 170 & 160 & 150 & 140 & 130 & 120 & 110 & 100 & 90 & 80 & 70 & 60 & 50 & 40 & 30 & 20 & 10 & 0 & -10 & \end{array}$

${ }^{13} \mathrm{C}$ NMR Compound $1 \mathbf{b}\left(75 \mathrm{MHz}, \mathrm{CDCl}_{3}\right)$ 


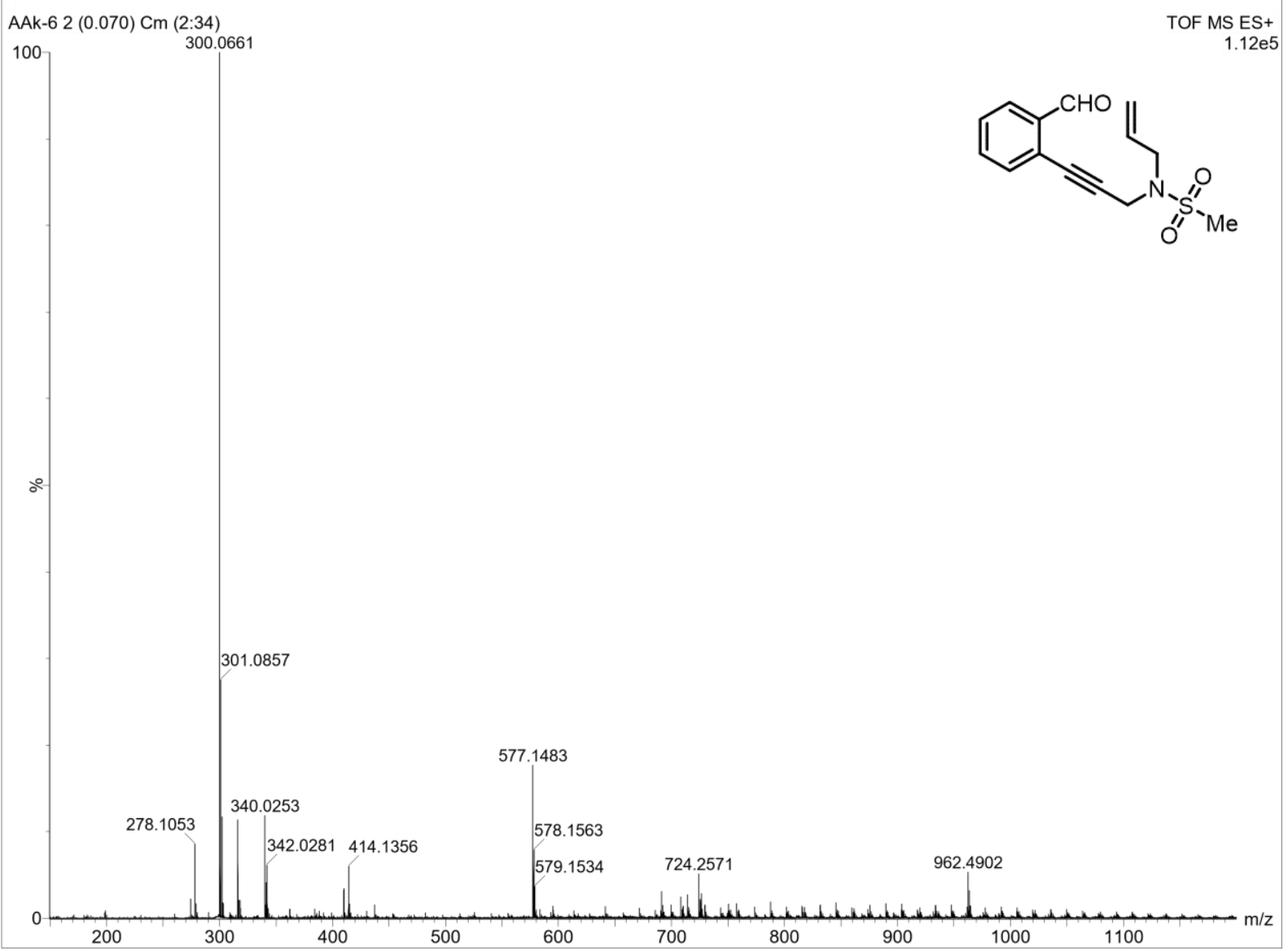

Mass-ESI Compound $\mathbf{1 b}$ 

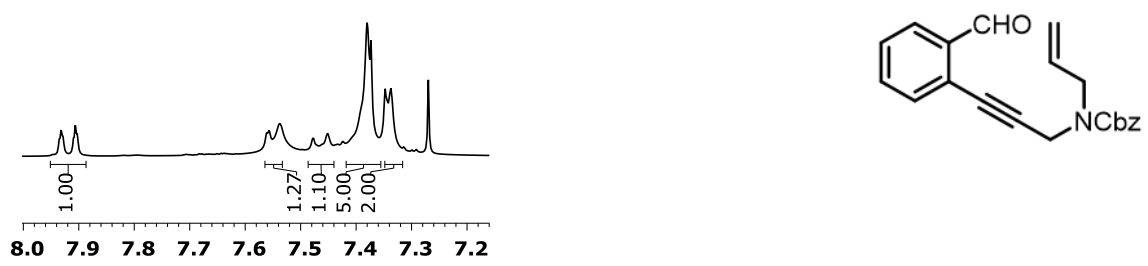

$\begin{array}{llllllllll}8.0 & 7.9 & 7.8 & 7.7 & 7.6 & 7.5 & 7.4 & 7.3 & 7.2\end{array}$

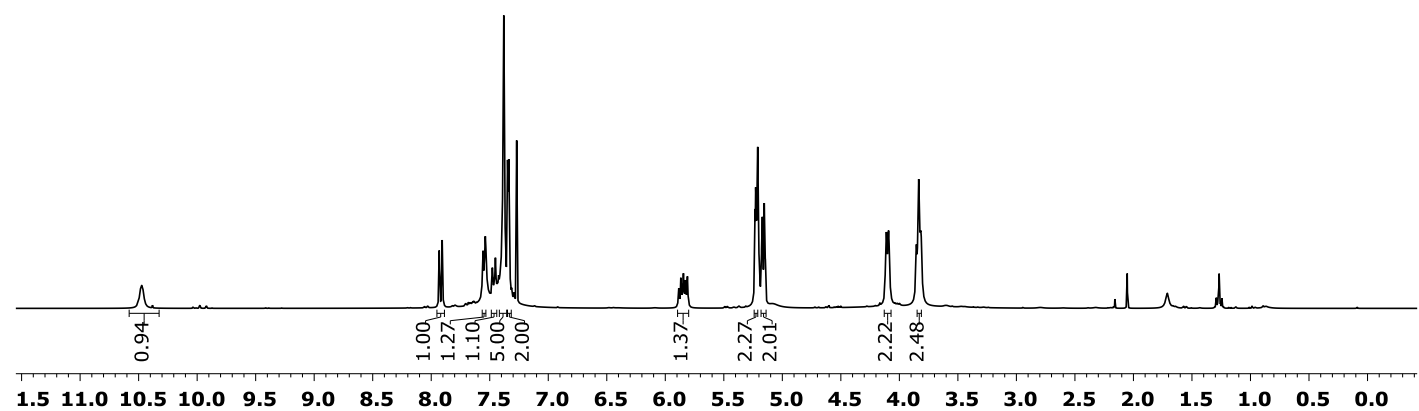

${ }^{1} \mathrm{H}$ NMR Compound 1c $\left(300 \mathrm{MHz}, \mathrm{CDCl}_{3}\right)$

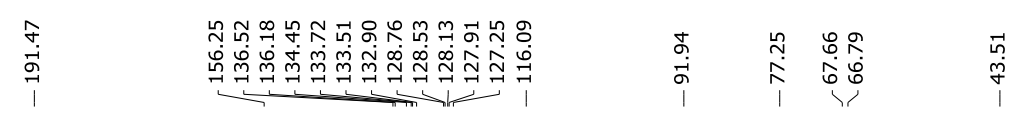
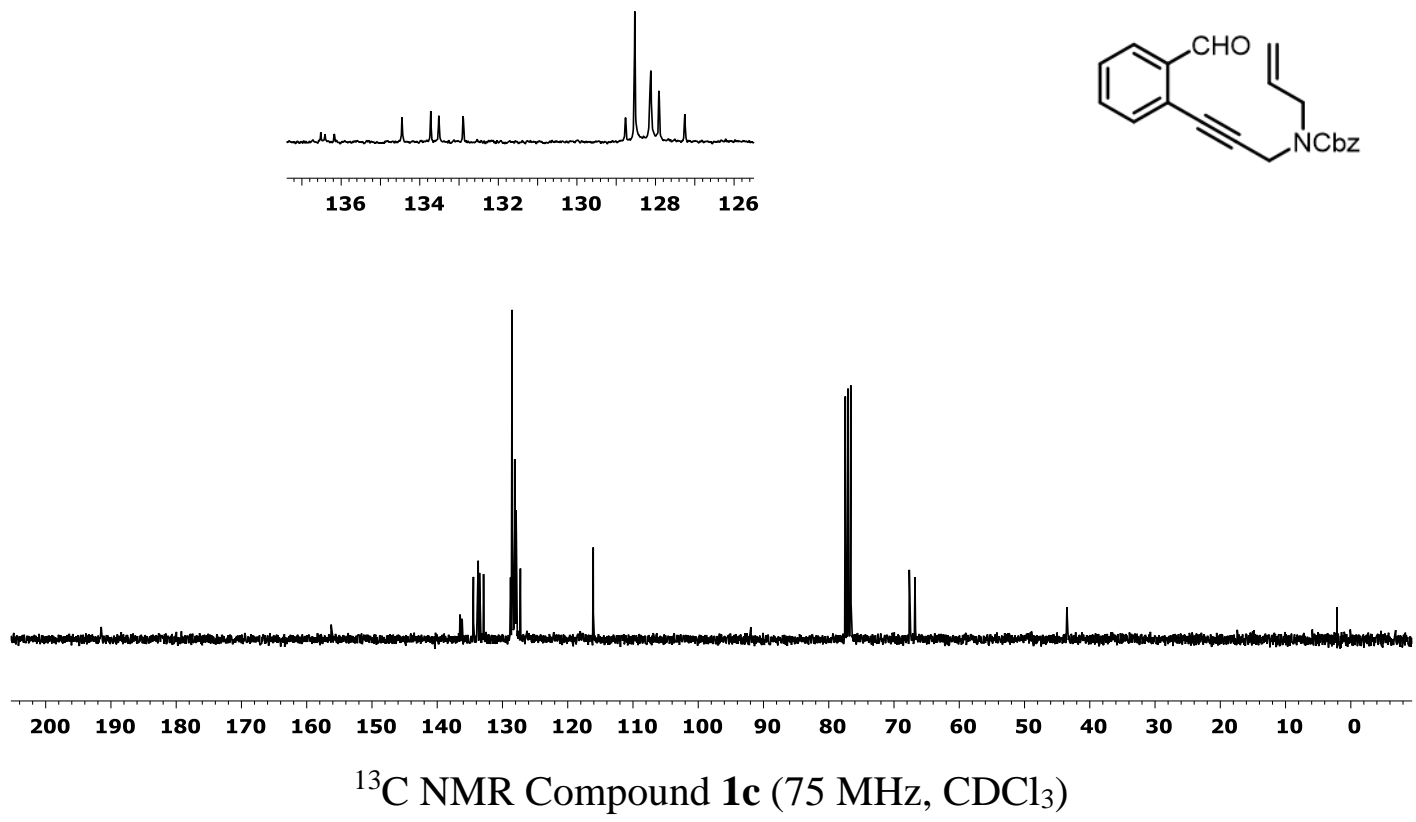
krbta52shr2\#1 RT: $0.02 \quad$ AV: $1 \quad \mathrm{NL}: 4.79 \mathrm{E}$

T: FTMS + p ESI Full ms [100.00-700.00]

$$
\mathrm{C}_{21} \mathrm{H}_{20} \mathrm{O}_{3} \mathrm{~N}=334.1438
$$

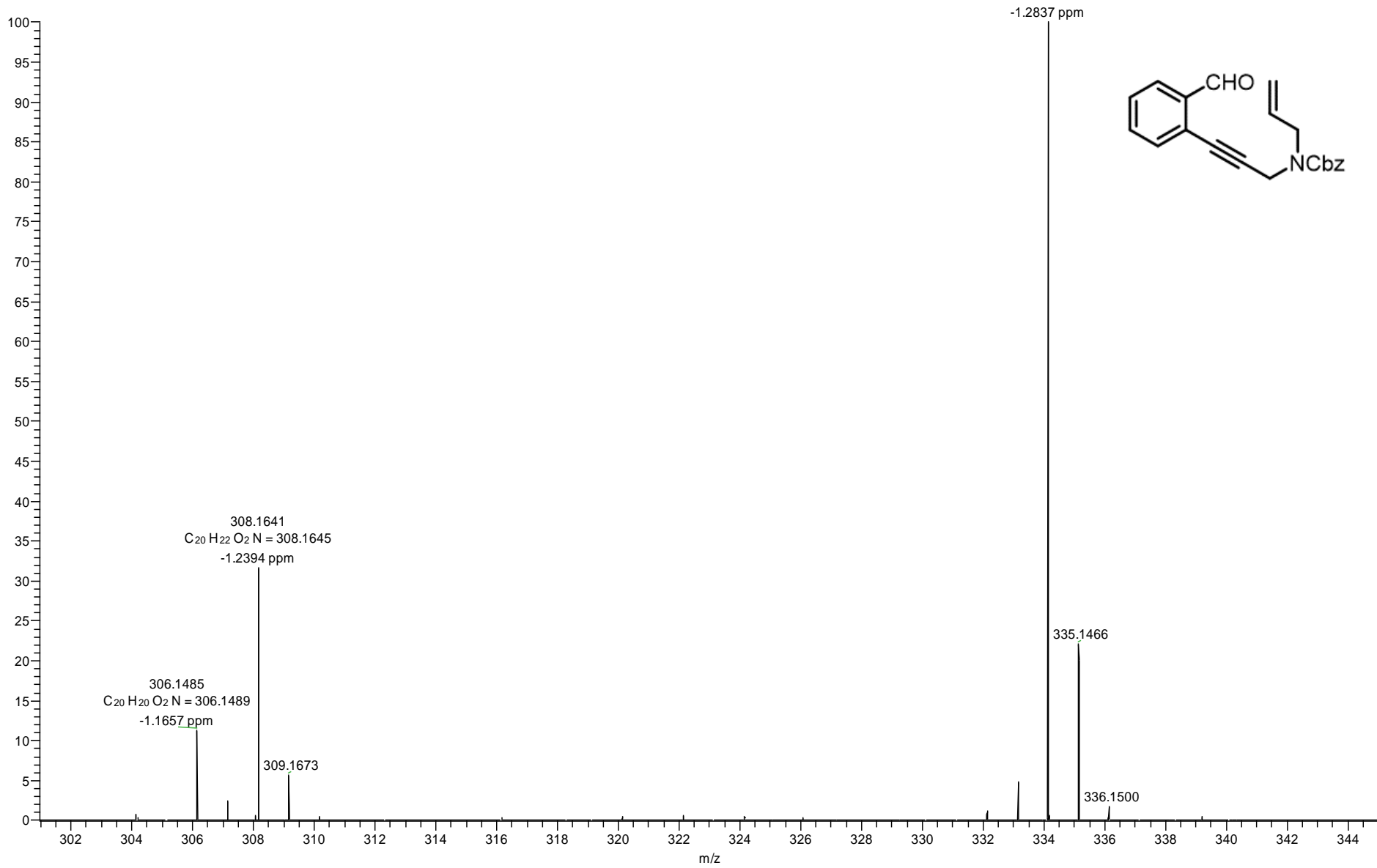

HRMS-ESI Compound 1c 

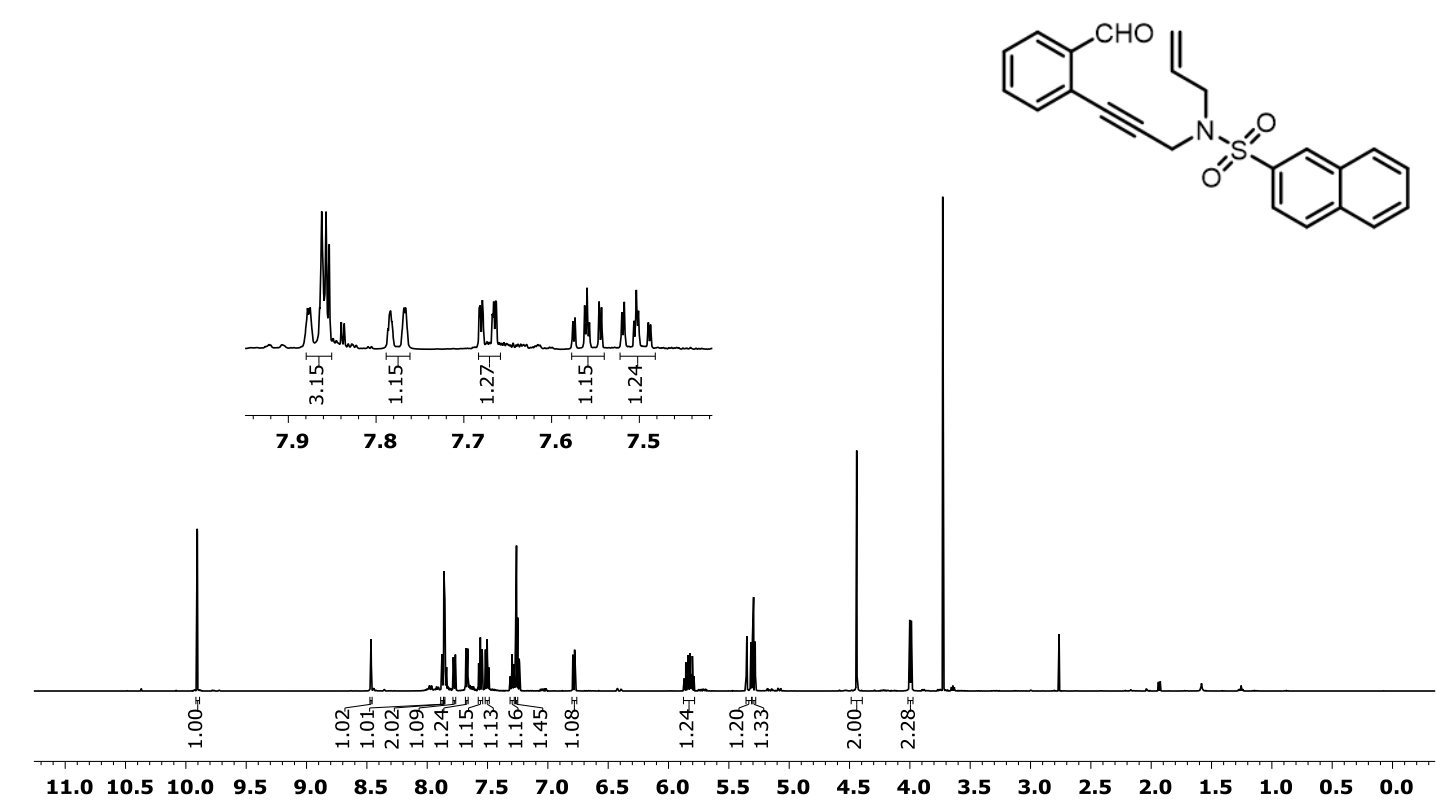

${ }^{1} \mathrm{H}$ NMR Compound $1 d\left(500 \mathrm{MHz}, \mathrm{CDCl}_{3}\right)$
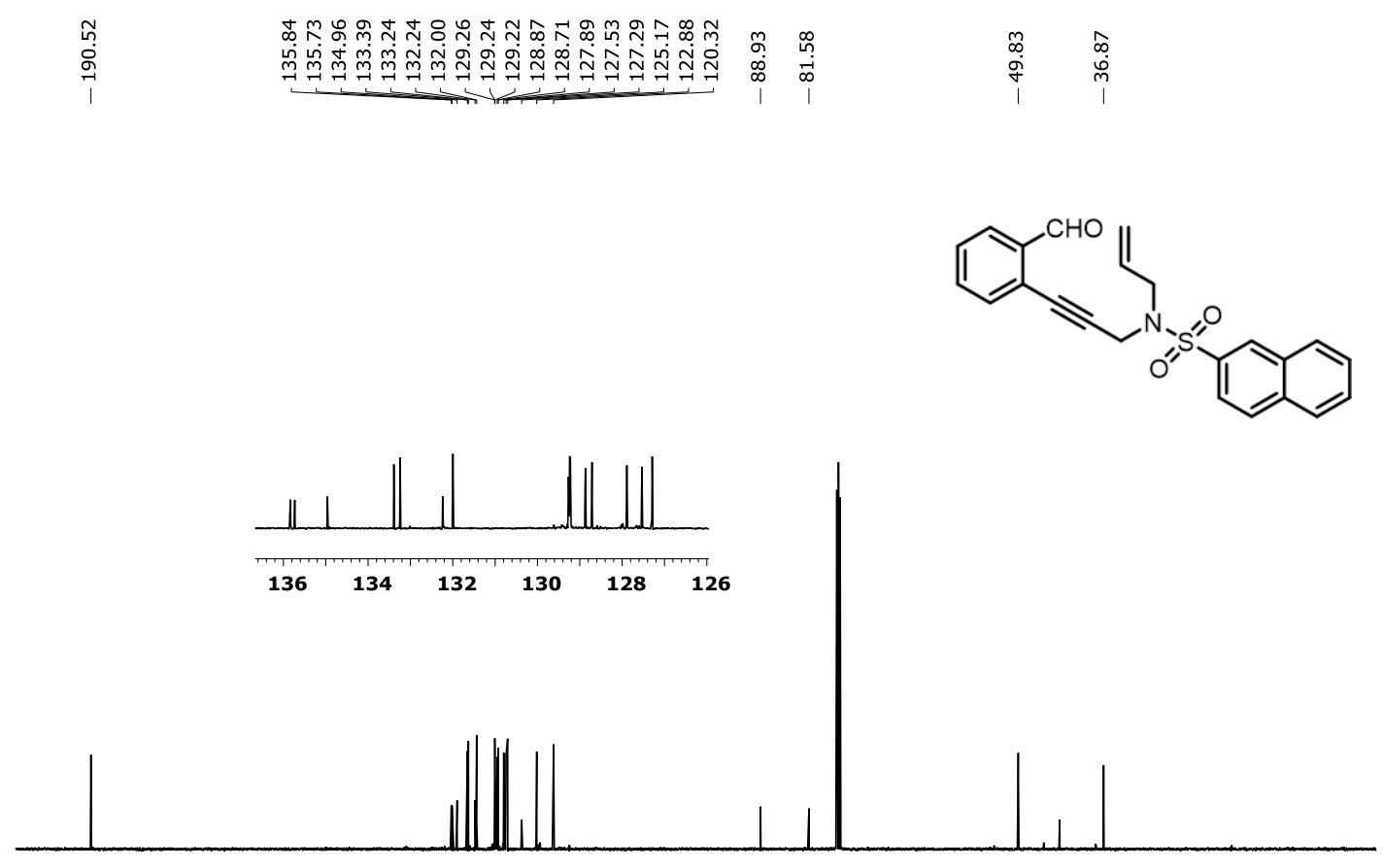

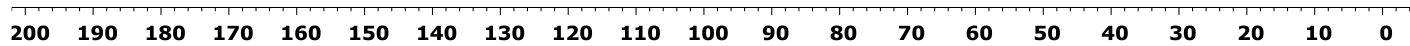

${ }^{13} \mathrm{C}$ NMR Compound $\mathbf{1 d}\left(125 \mathrm{MHz}, \mathrm{CDCl}_{3}\right)$ 


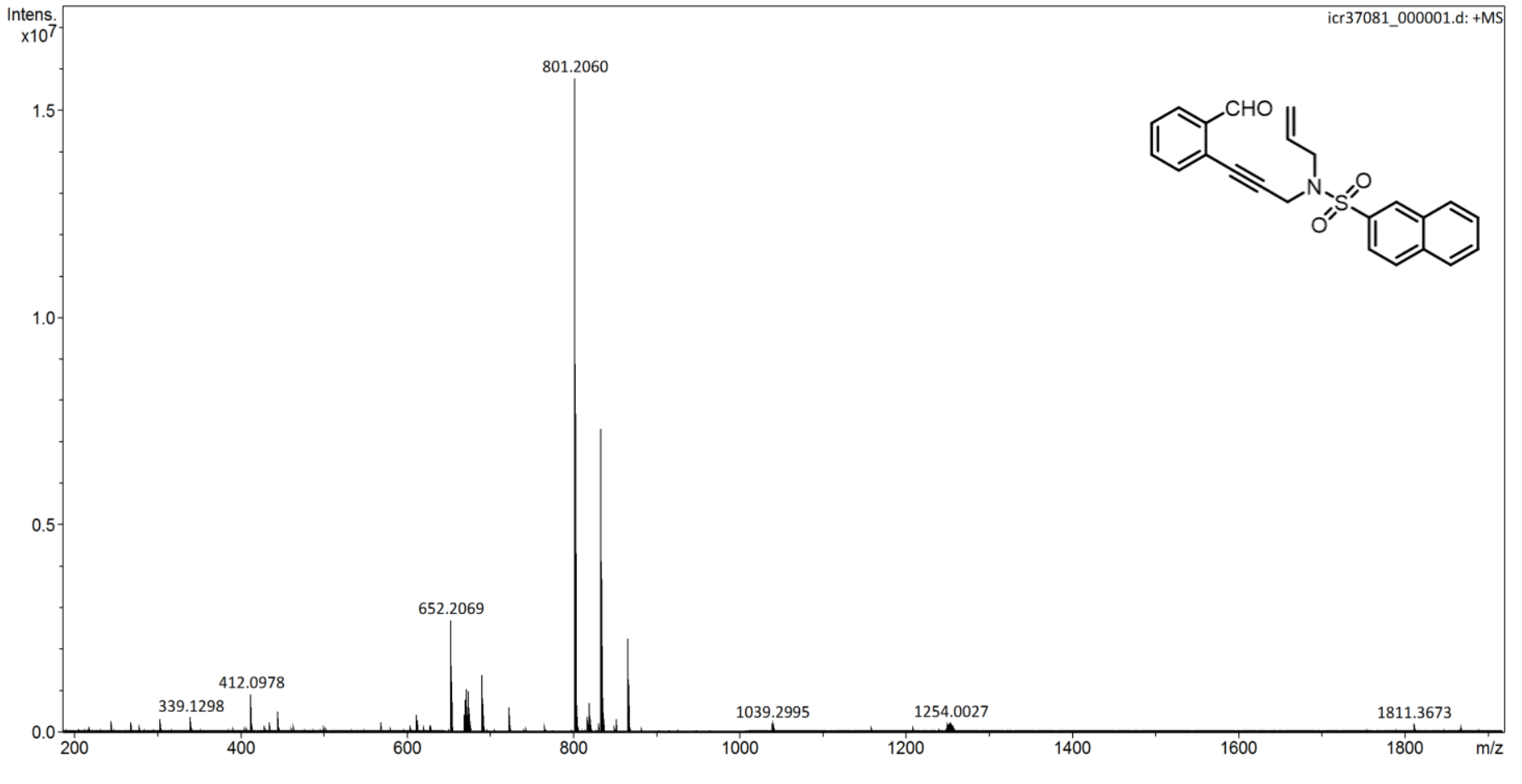

HRMS-ESI Compound 1d

\begin{tabular}{|c|c|c|c|c|c|c|c|}
\hline Meas. $\mathrm{m} / \mathrm{z}$ & Ion Formula & $\mathrm{m} / \mathrm{z}$ & err [ppm] & mSigma & $\mathrm{rdb}$ & $\mathrm{e}^{-}$Conf & N-Rule \\
\hline \multirow[t]{2}{*}{390.1158} & $\mathrm{C} 23 \mathrm{H} 20 \mathrm{NO} 3 \mathrm{~S}$ & 390.1158 & 0.0 & 26.2 & 14.5 & even & ok \\
\hline & C29H14N2 & 390.1151 & -1.7 & 26.7 & 24.0 & odd & \\
\hline \multirow[t]{6}{*}{412.0978} & $\mathrm{C} 23 \mathrm{H} 19 \mathrm{NNaO} 3 \mathrm{~S}$ & 412.0978 & -0.1 & 8.2 & 14.5 & even & \\
\hline & $\mathrm{C} 22 \mathrm{H} 20 \mathrm{O} 6 \mathrm{~S}$ & 412.0975 & -0.7 & 9.3 & 13.0 & odd & \\
\hline & $\mathrm{C} 16 \mathrm{H} 18 \mathrm{~N} 3 \mathrm{O} 10$ & 412.0987 & 2.1 & 32.4 & 9.5 & even & \\
\hline & $\mathrm{C} 16 \mathrm{H} 21 \mathrm{NaO} 11$ & 412.0976 & -0.5 & 35.9 & 6.0 & odd & \\
\hline & $\mathrm{C} 29 \mathrm{H} 13 \mathrm{~N} 2 \mathrm{Na}$ & 412.0971 & -1.8 & 36.3 & 24.0 & odd & \\
\hline & C21H14N7OS & 412.0975 & -0.8 & 116.3 & 18.5 & even & \\
\hline \multirow[t]{7}{*}{801.2060} & $\mathrm{C} 46 \mathrm{H} 38 \mathrm{~N} 2 \mathrm{NaO} 6 \mathrm{~S} 2$ & 801.2063 & 0.5 & 22.2 & 28.5 & even & \\
\hline & $\mathrm{C} 46 \mathrm{H} 35 \mathrm{~N} 5 \mathrm{O} 5 \mathrm{~S} 2$ & 801.2074 & 1.8 & 25.0 & 32.0 & odd & \\
\hline & $\mathrm{C} 46 \mathrm{H} 30 \mathrm{~N} 6 \mathrm{NaO} 7$ & 801.2068 & 1.1 & 29.9 & 34.5 & even & \\
\hline & $\mathrm{C} 50 \mathrm{H} 30 \mathrm{~N} 6 \mathrm{NaO} 2 \mathrm{~S}$ & 801.2043 & -2.1 & 42.9 & 38.5 & even & \\
\hline & C51H33N2O6S & 801.2054 & -0.7 & 46.8 & 36.5 & even & \\
\hline & C52H32N3NaO3S & 801.2057 & -0.4 & 50.4 & 38.0 & odd & \\
\hline & $\mathrm{C} 54 \mathrm{H} 34 \mathrm{NaO} 4 \mathrm{~S}$ & 801.2070 & 1.3 & 58.3 & 37.5 & even & \\
\hline
\end{tabular}



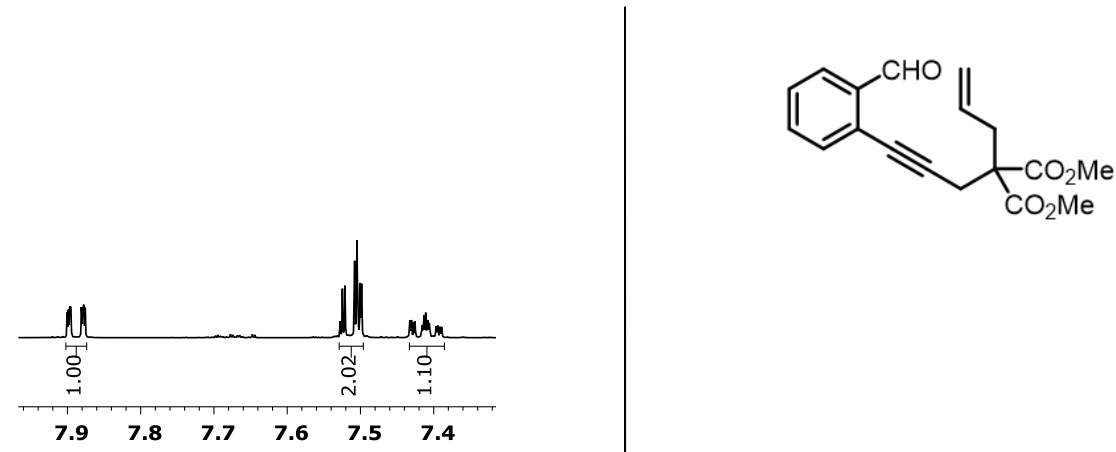

$\mathrm{O}_{2} \mathrm{Me}$

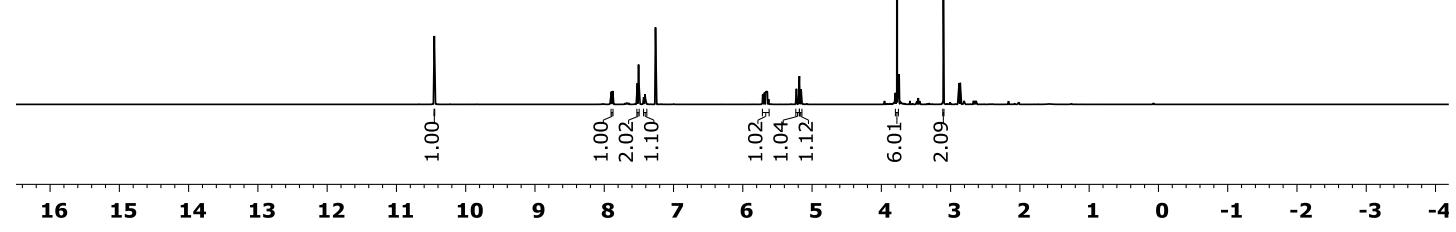

${ }^{1} \mathrm{H}$ NMR Compound $1 \mathrm{e}\left(400 \mathrm{MHz}, \mathrm{CDCl}_{3}\right)$

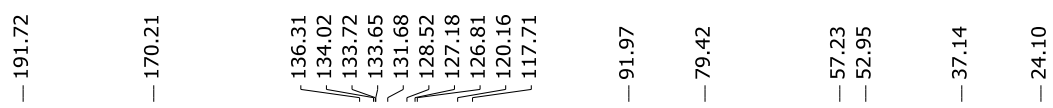
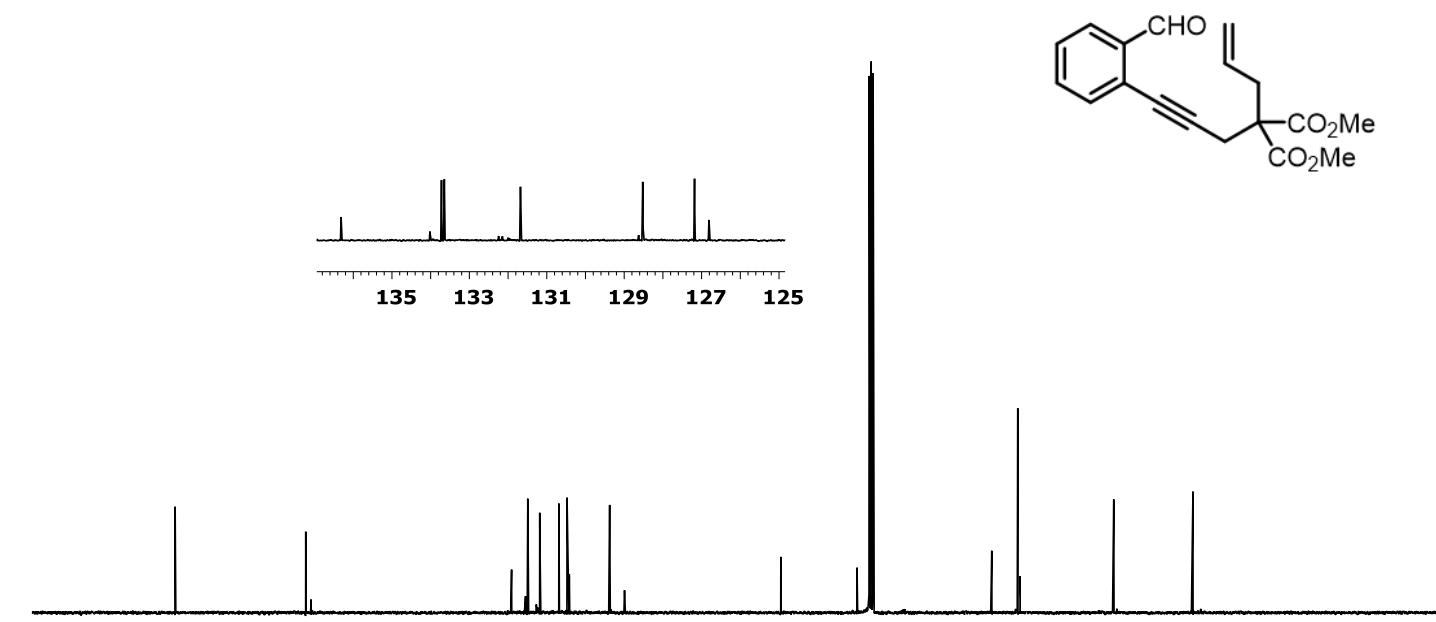

$\begin{array}{lllllllllllllllllllllllllllll}210 & 200 & 190 & 180 & 170 & 160 & 150 & 140 & 130 & 120 & 110 & 100 & 90 & 80 & 70 & 60 & 50 & 40 & 30 & 20 & 10 & 0 & -10\end{array}$

${ }^{13} \mathrm{C}$ NMR Compound $1 \mathrm{e}\left(100 \mathrm{MHz}, \mathrm{CDCl}_{3}\right)$ 


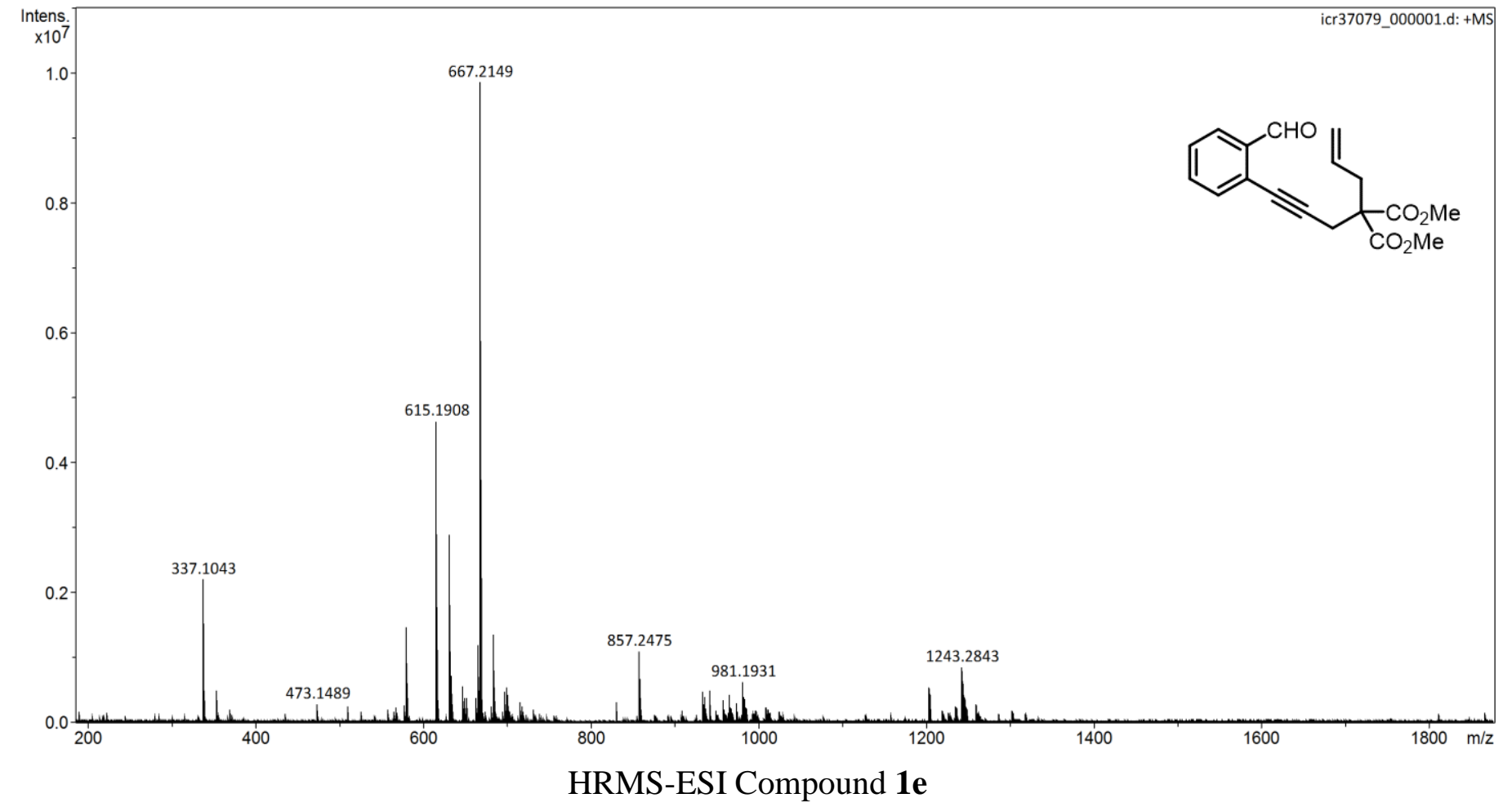

\begin{tabular}{|c|c|c|c|c|c|c|c|}
\hline Meas. m/z & Ion Formula & $\mathrm{m} / \mathrm{z}$ & err [ppm] & mSigma & $\mathrm{rdb}$ & $\mathrm{e}^{-}$Conf & N-Rule \\
\hline \multirow[t]{2}{*}{315.1229} & C18H19O5 & 315.1227 & -0.5 & n.a. & 9.5 & even & ok \\
\hline & C17H13N7 & 315.1227 & -0.5 & n.a. & 15.0 & odd & ok \\
\hline \multirow[t]{3}{*}{337.1043} & $\mathrm{C} 18 \mathrm{H} 18 \mathrm{NaO} 5$ & 337.1046 & 1.0 & 13.2 & 9.5 & even & ok \\
\hline & $\mathrm{C} 17 \mathrm{H} 12 \mathrm{~N} 7 \mathrm{Na}$ & 337.1046 & 1.0 & 20.3 & 15.0 & odd & ok \\
\hline & C16H13N6O3 & 337.1044 & 0.2 & 22.4 & 13.5 & even & ok \\
\hline \multirow[t]{5}{*}{651.2202} & $\mathrm{C} 37 \mathrm{H} 32 \mathrm{~N} 4 \mathrm{NaO} 6$ & 651.2214 & 1.8 & 12.4 & 23.5 & even & ok \\
\hline & $\mathrm{C} 36 \mathrm{H} 33 \mathrm{~N} 3 \mathrm{O} 9$ & 651.2211 & 1.4 & 14.9 & 22.0 & odd & ok \\
\hline & $\mathrm{C} 36 \mathrm{H} 36 \mathrm{NaO} 10$ & 651.2201 & -0.2 & 15.6 & 18.5 & even & ok \\
\hline & $\mathrm{C} 36 \mathrm{H} 26 \mathrm{~N} 11 \mathrm{NaO}$ & 651.2214 & 1.8 & 25.5 & 29.0 & odd & ok \\
\hline & $\mathrm{C} 48 \mathrm{H} 29 \mathrm{NO} 2$ & 651.2193 & -1.4 & 55.2 & 35.0 & odd & ok \\
\hline
\end{tabular}



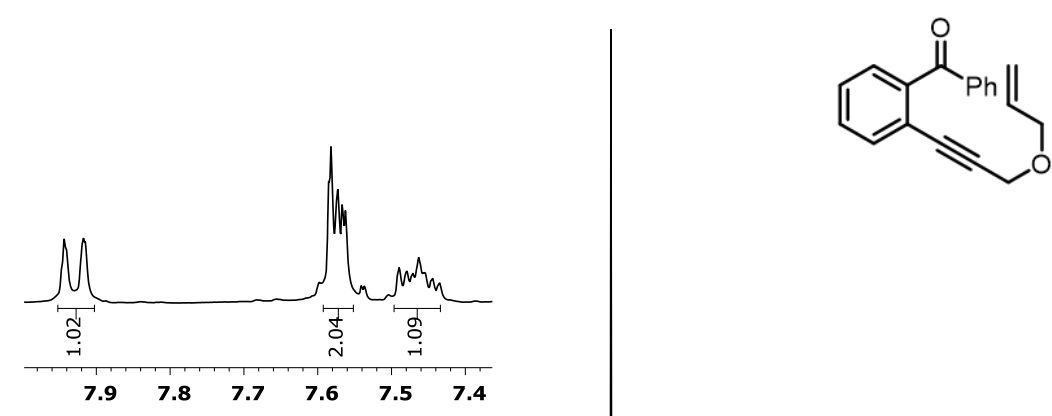

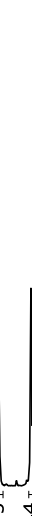

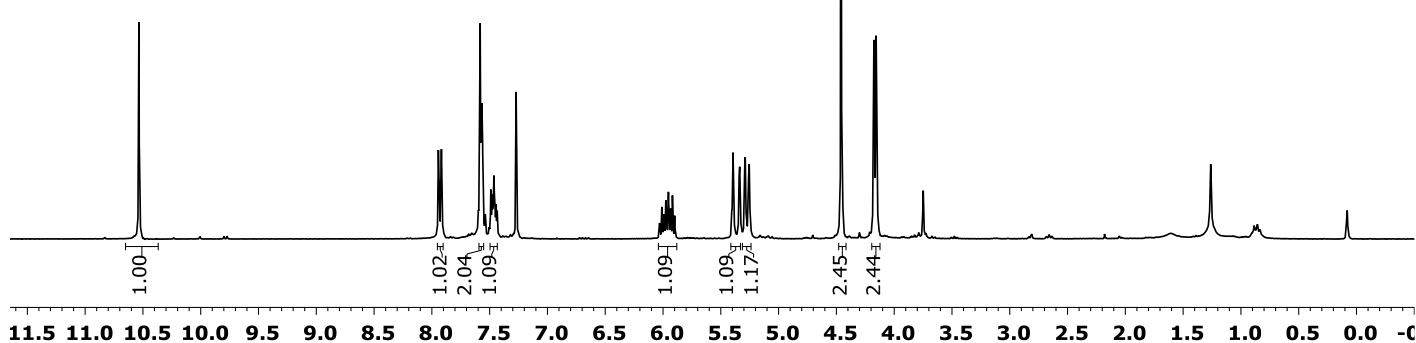

${ }^{1} \mathrm{H}$ NMR Compound $1 f\left(300 \mathrm{MHz}, \mathrm{CDCl}_{3}\right)$

菖
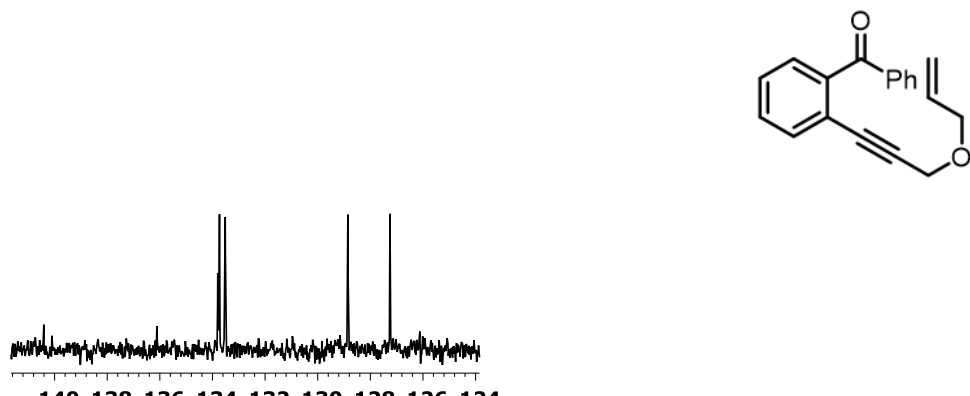

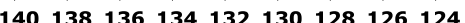

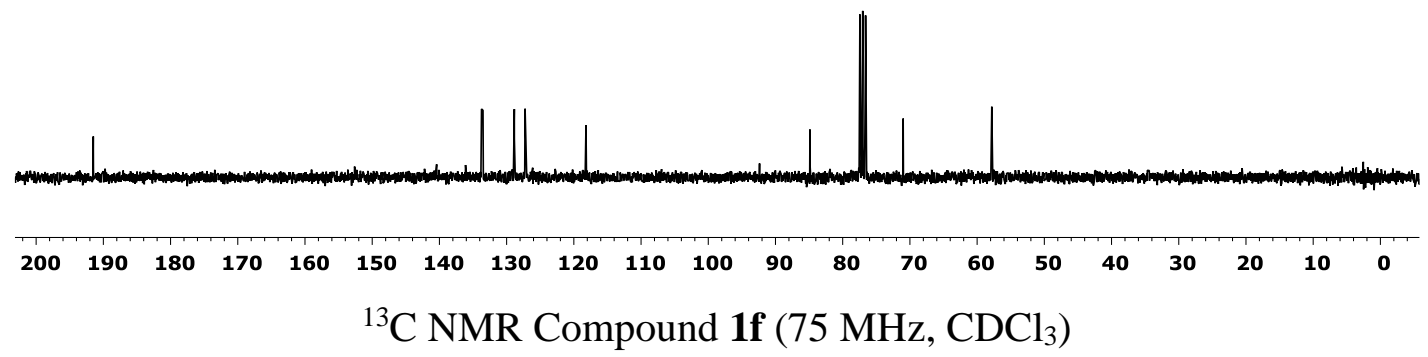


hobta30shr1 \#1 RT: 0.02 AV: 1 NL: $1.97 E 6$

T: FTMS + p ESI Full lock ms [100.00-800.00]

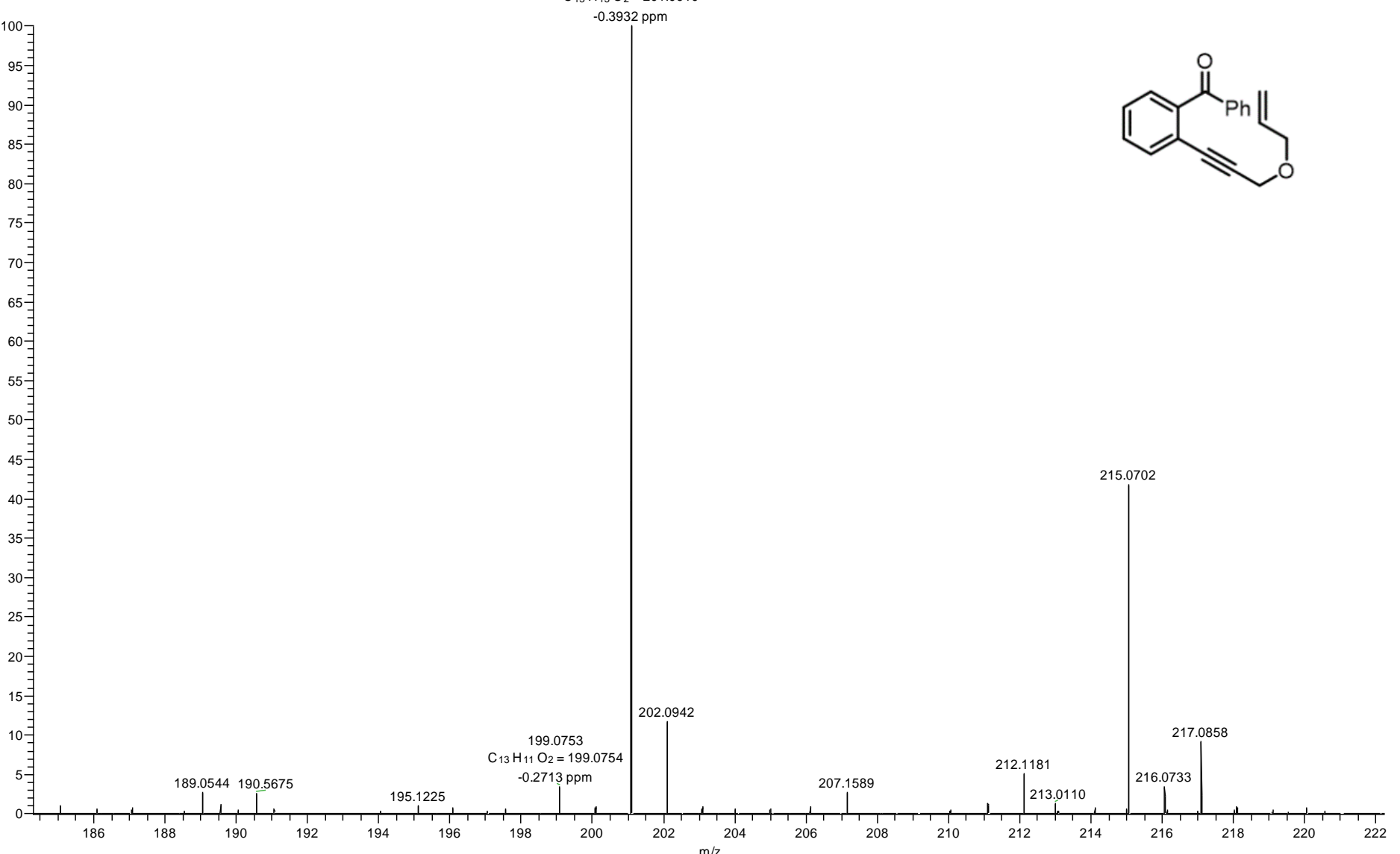

HRMS-ESI Compound 1f 

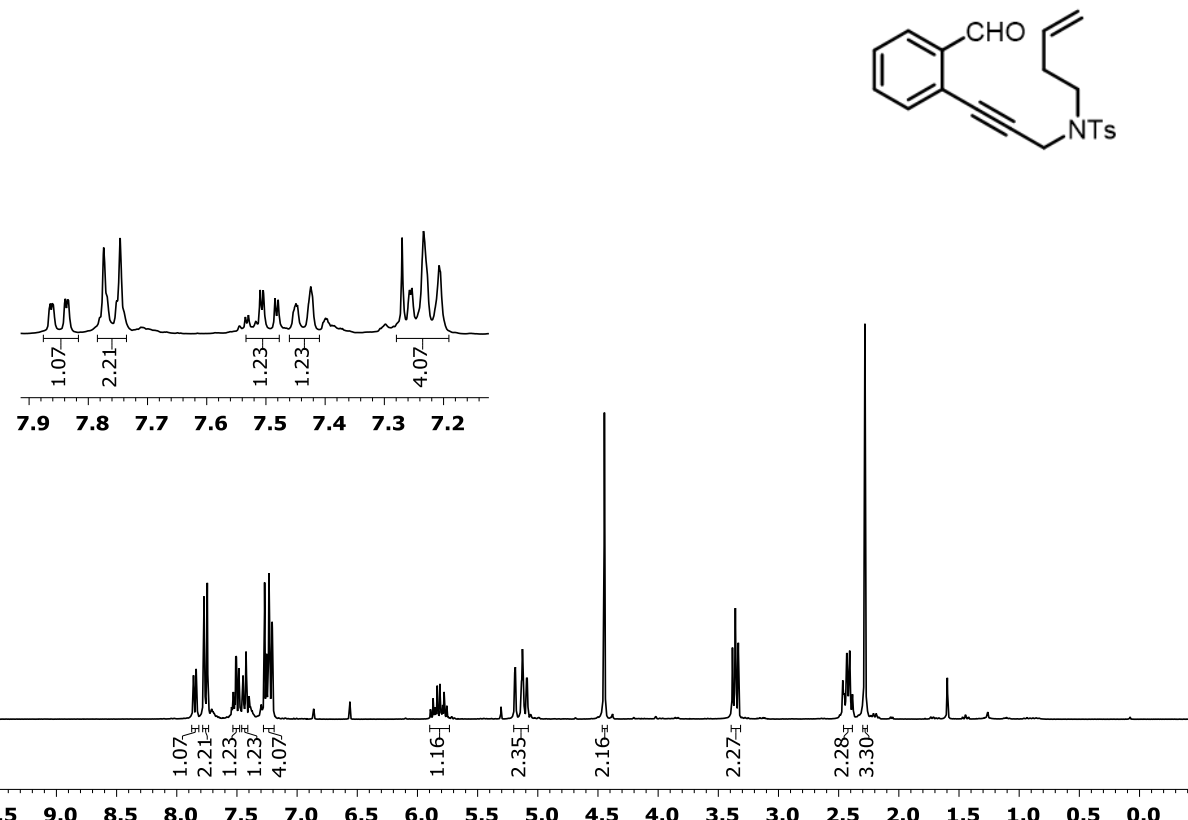

${ }^{1} \mathrm{H}$ NMR Compound $1 \mathrm{~g}\left(300 \mathrm{MHz}, \mathrm{CDCl}_{3}\right)$

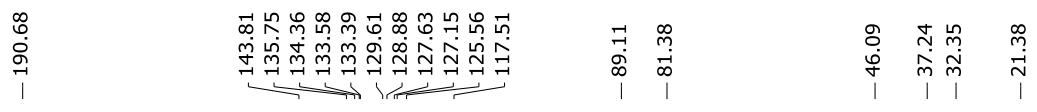
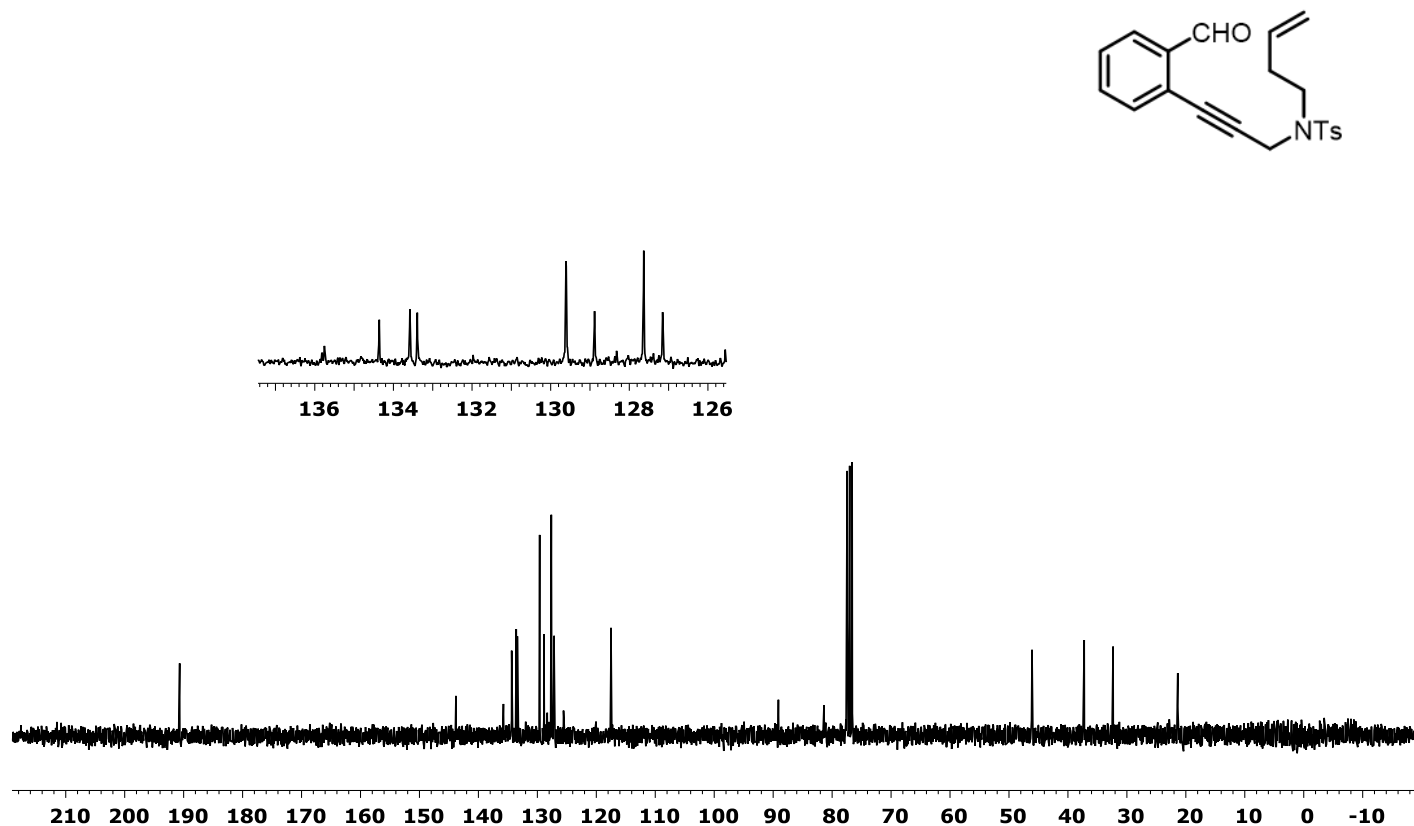

${ }^{13} \mathrm{C}$ NMR Compound $1 \mathrm{~g}\left(75 \mathrm{MHz}, \mathrm{CDCl}_{3}\right)$ 
hobta28shr2 \#1 RT: 0.02 AV: 1 NL: 1.50E6 T: FTMS + p ESI Full lock ms [100.00-700.00]

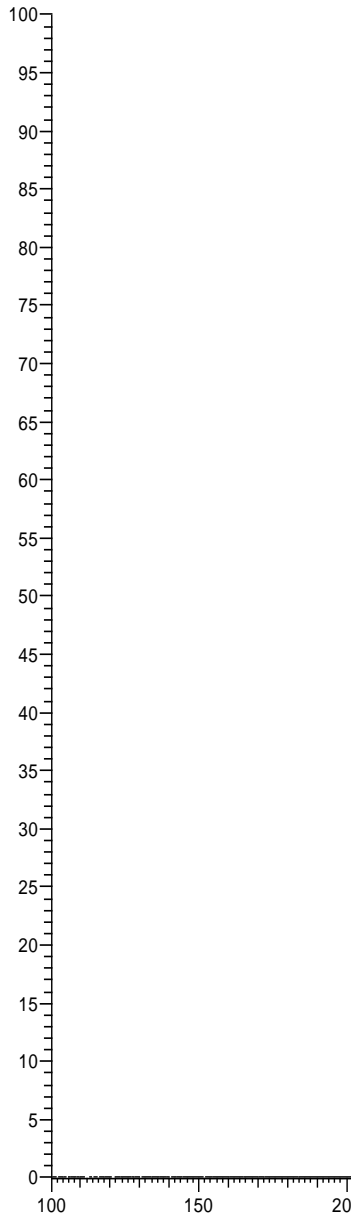

$-0.2229 \mathrm{ppm}$
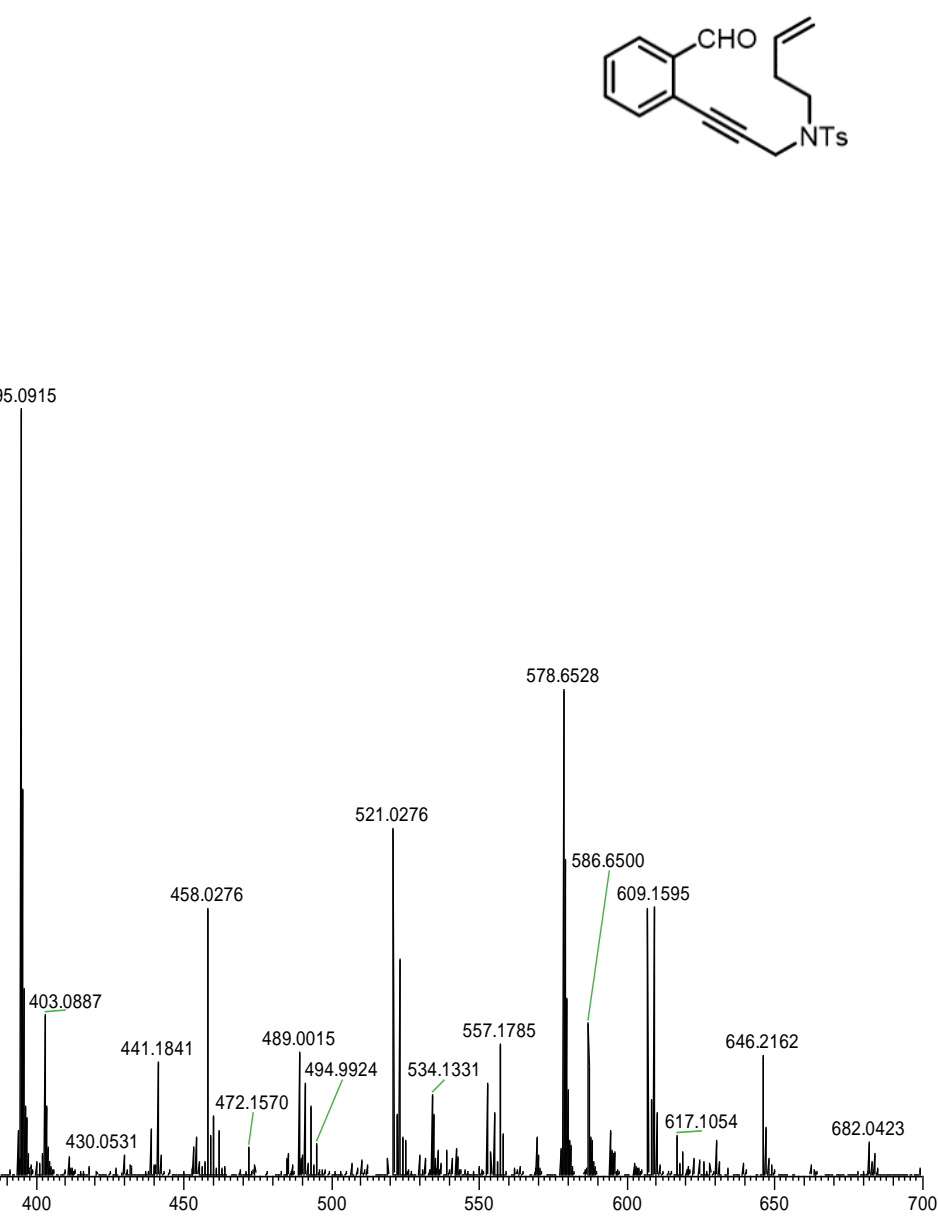

HRMS-ESI Compound 1g 


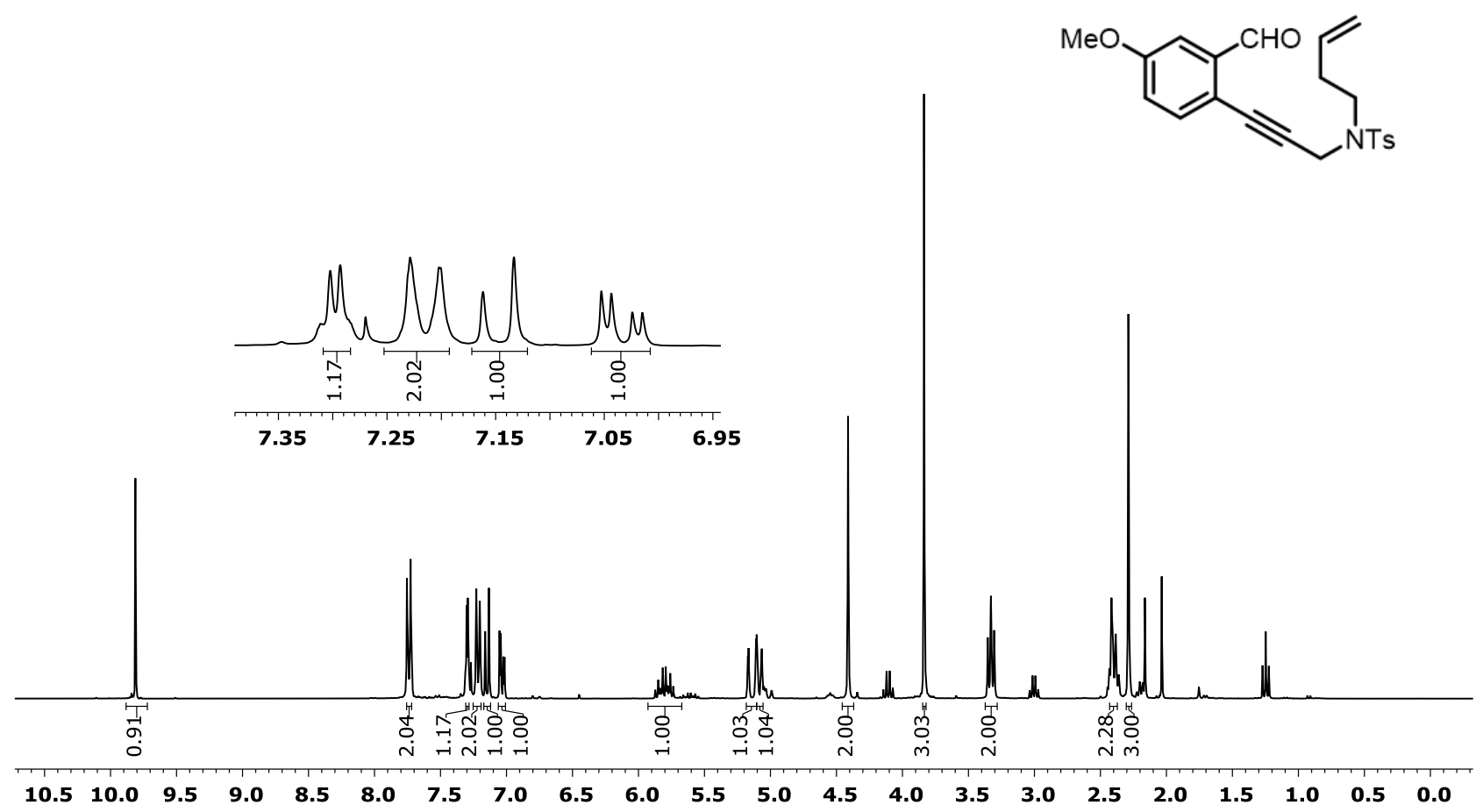

${ }^{1} \mathrm{H}$ NMR Compound $\mathbf{1 h}\left(300 \mathrm{MHz}, \mathrm{CDCl}_{3}\right)$

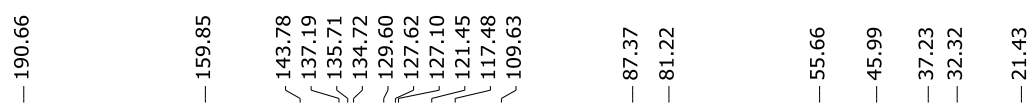
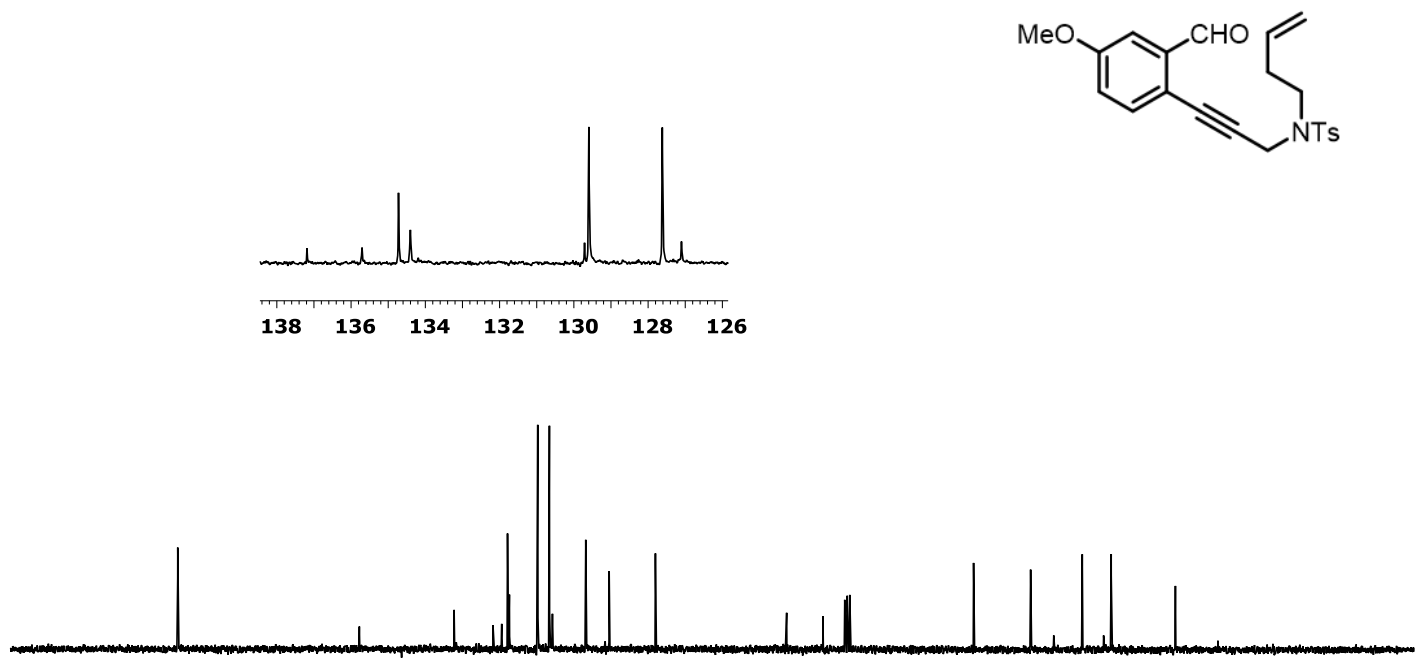

$\begin{array}{lllllllllllllllllllllll}210 & 200 & 190 & 180 & 170 & 160 & 150 & 140 & 130 & 120 & 110 & 100 & 90 & 80 & 70 & 60 & 50 & 40 & 30 & 20 & 10 & 0 & -10\end{array}$

${ }^{13} \mathrm{C}$ NMR Compound $1 \mathbf{h}\left(75 \mathrm{MHz}, \mathrm{CDCl}_{3}\right)$ 


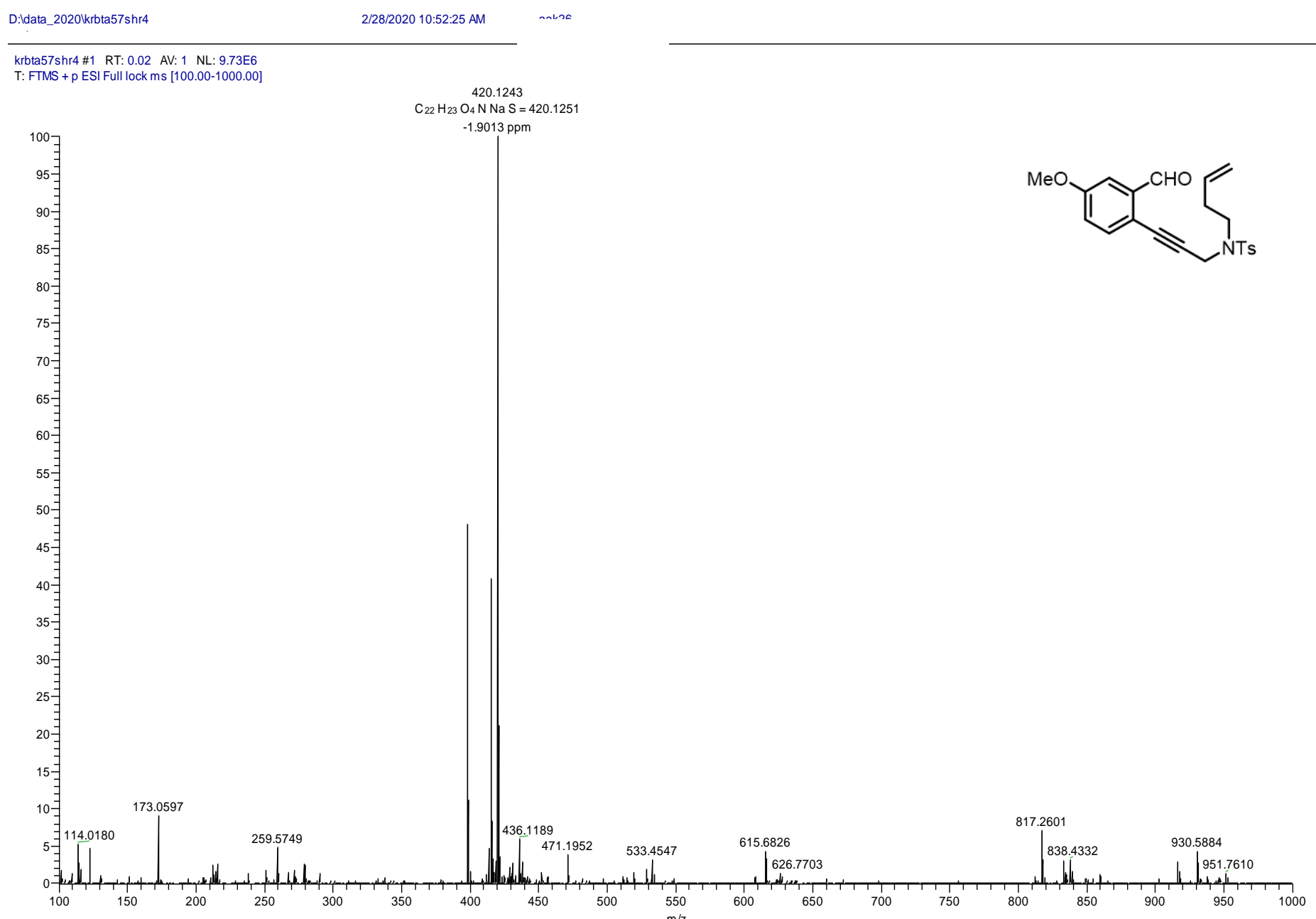

\section{HRMS-ESI Compound 1h}



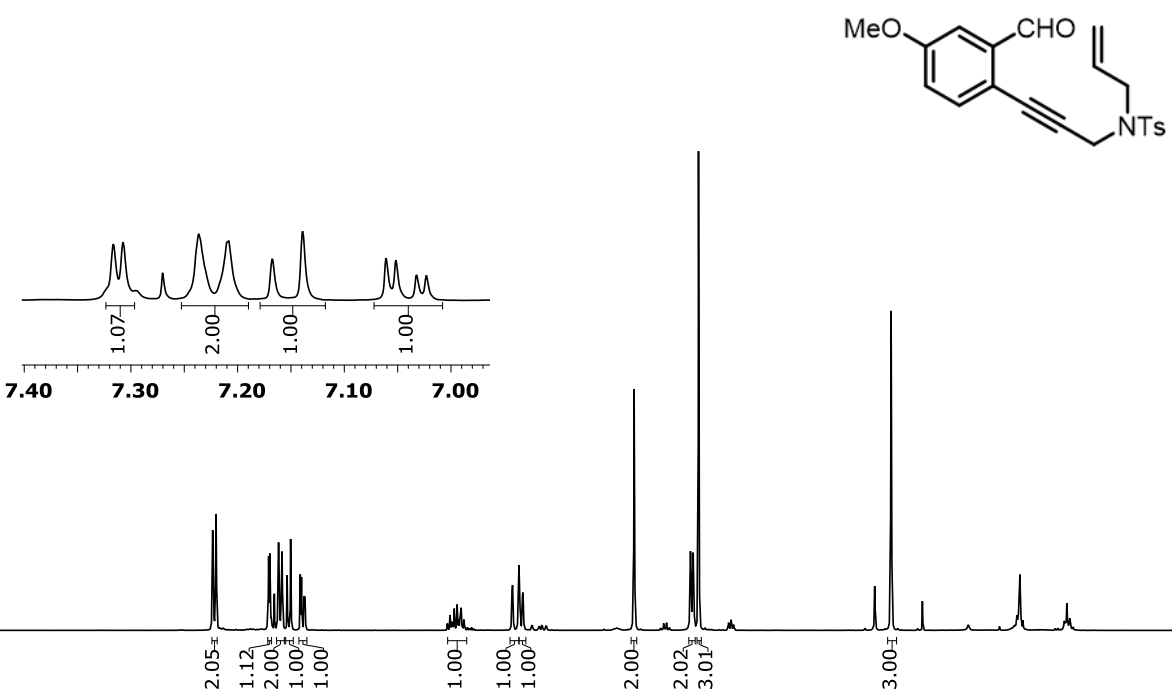

$\begin{array}{llllllllllllllllllllllll}1.0 & 10.5 & 10.0 & 9.5 & 9.0 & 8.5 & 8.0 & 7.5 & 7.0 & 6.5 & 6.0 & 5.5 & 5.0 & 4.5 & 4.0 & 3.5 & 3.0 & 2.5 & 2.0 & 1.5 & 1.0 & 0.5 & 0.0\end{array}$

${ }^{1} \mathrm{H}$ NMR Compound $\mathbf{1 i}\left(300 \mathrm{MHz}, \mathrm{CDCl}_{3}\right)$

\begin{tabular}{|c|c|c|c|c|c|c|}
\hline 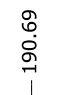 & $\begin{array}{l}\stackrel{0}{\infty} \\
\stackrel{0}{0} \\
\stackrel{0}{0} \\
1\end{array}$ & 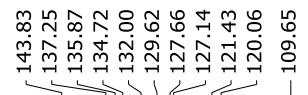 & 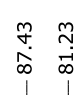 & & $\begin{array}{l}\text { 苟 } \\
\text { gे }\end{array}$ & $\begin{array}{l}\stackrel{m}{0} \\
\dot{p} \\
i\end{array}$ \\
\hline
\end{tabular}
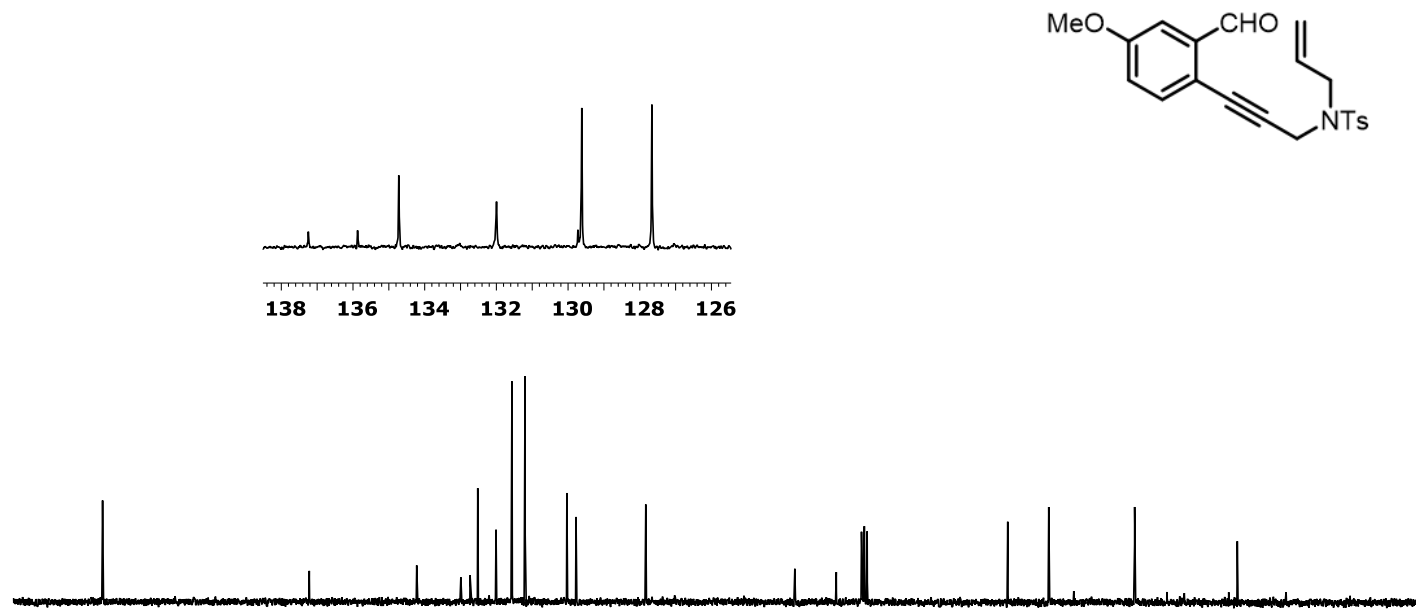

$\begin{array}{llllllllllllllllllllllllll}200 & 190 & 180 & 170 & 160 & 150 & 140 & 130 & 120 & 110 & 100 & 90 & 80 & 70 & 60 & 50 & 40 & 30 & 20 & 10 & 0\end{array}$

${ }^{13} \mathrm{C}$ NMR Compound $\mathbf{1 i}\left(75 \mathrm{MHz}, \mathrm{CDCl}_{3}\right)$ 


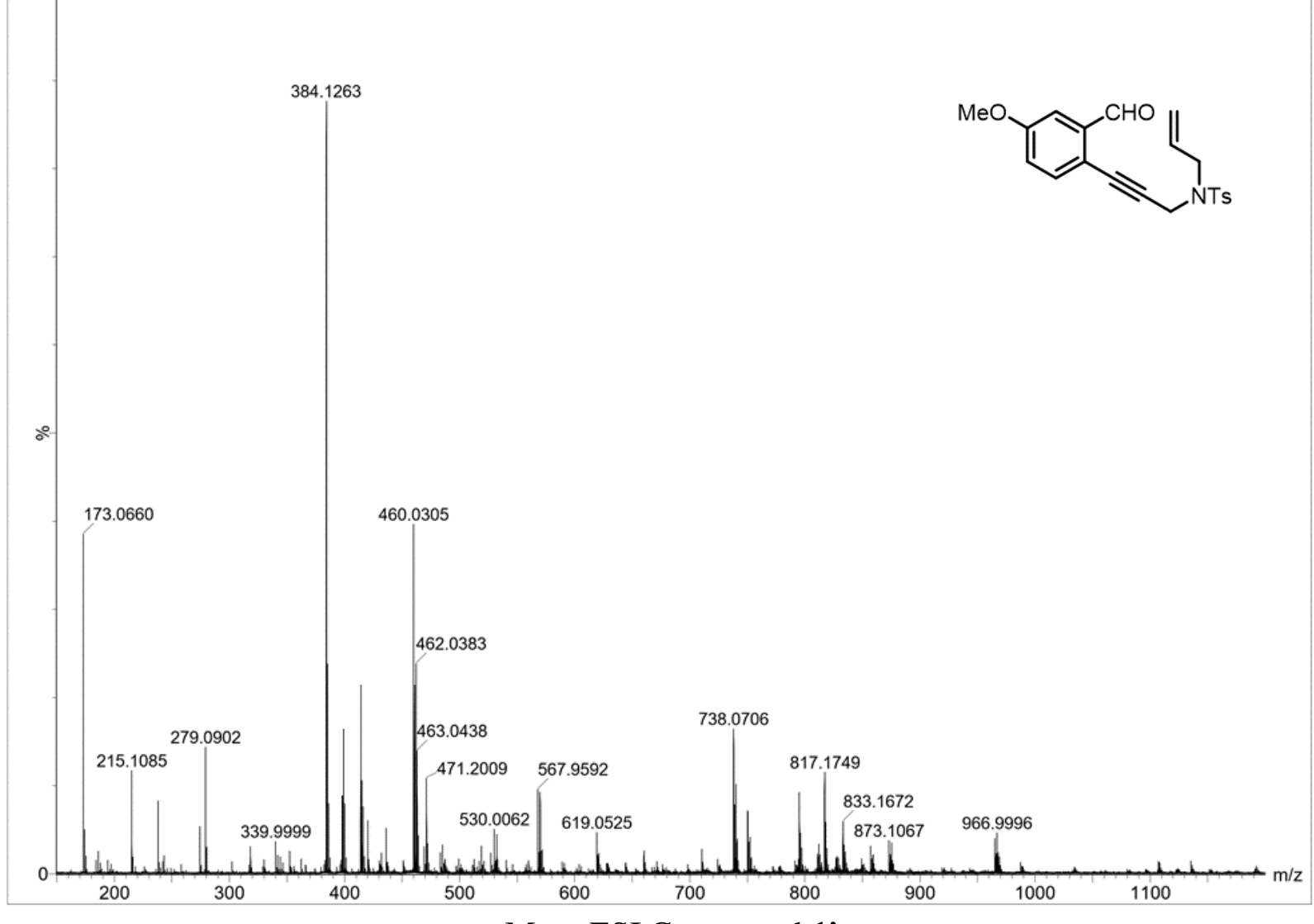

Mass-ESI Compound 1i 


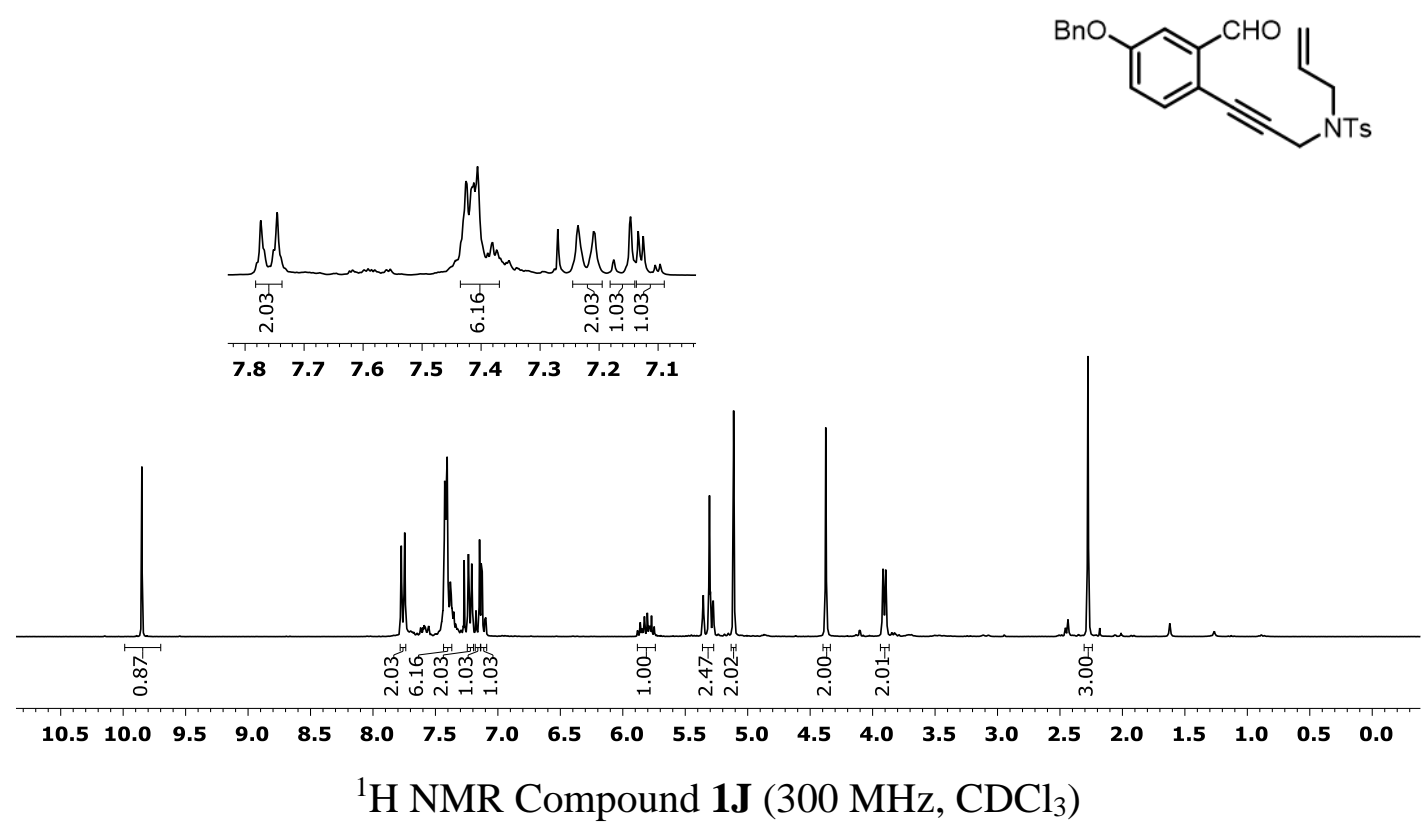

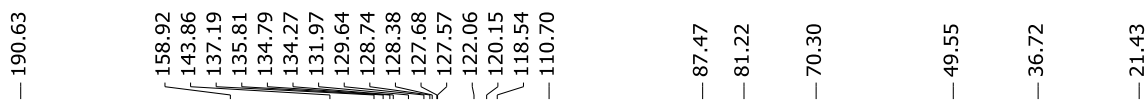

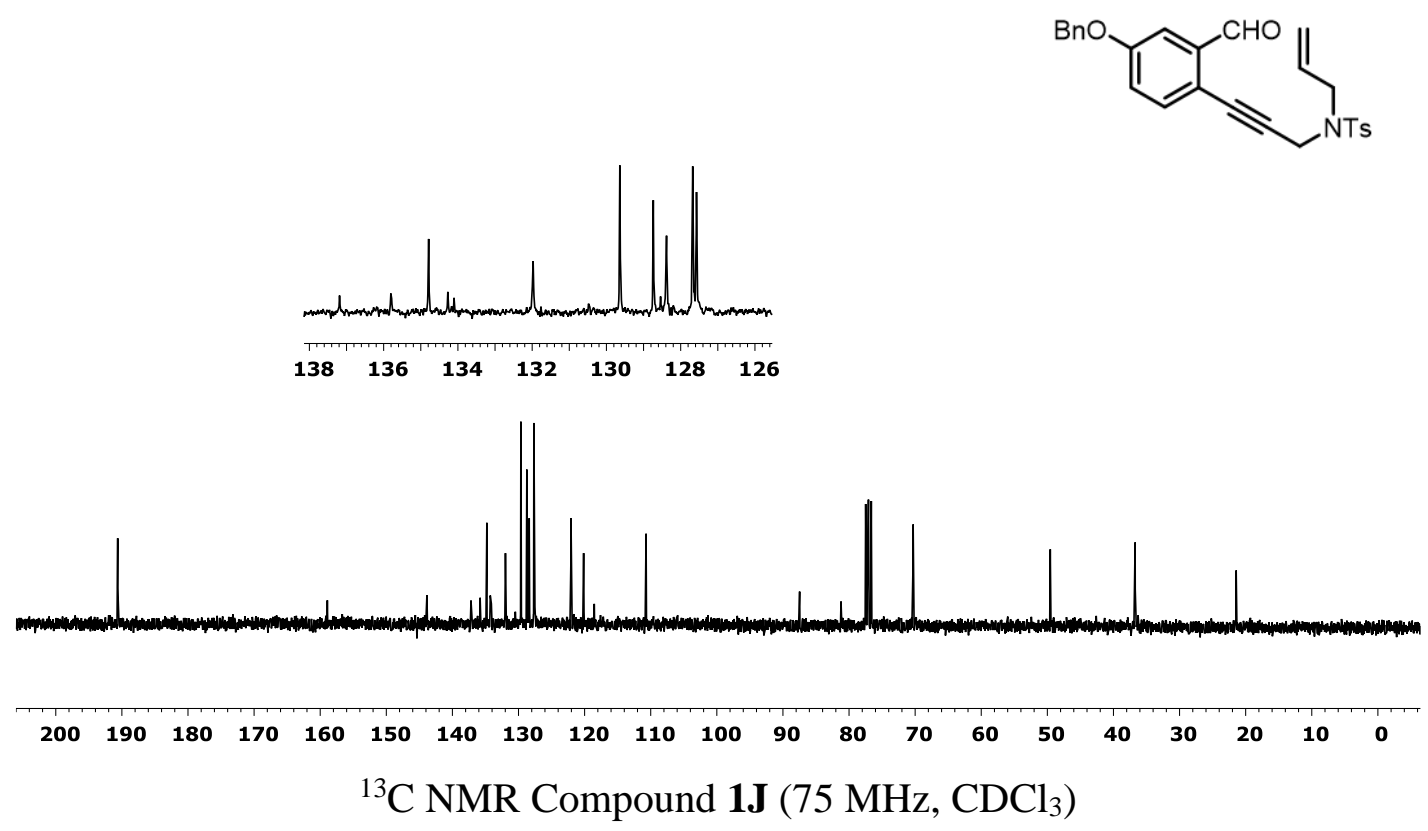


hobta32shr1 \#1 RT: $0.02 \quad$ AV: $1 \quad \mathrm{NL}: 2.17 \mathrm{E}$

T: FTMS + p ESI Full lock ms [100.00-800.00]
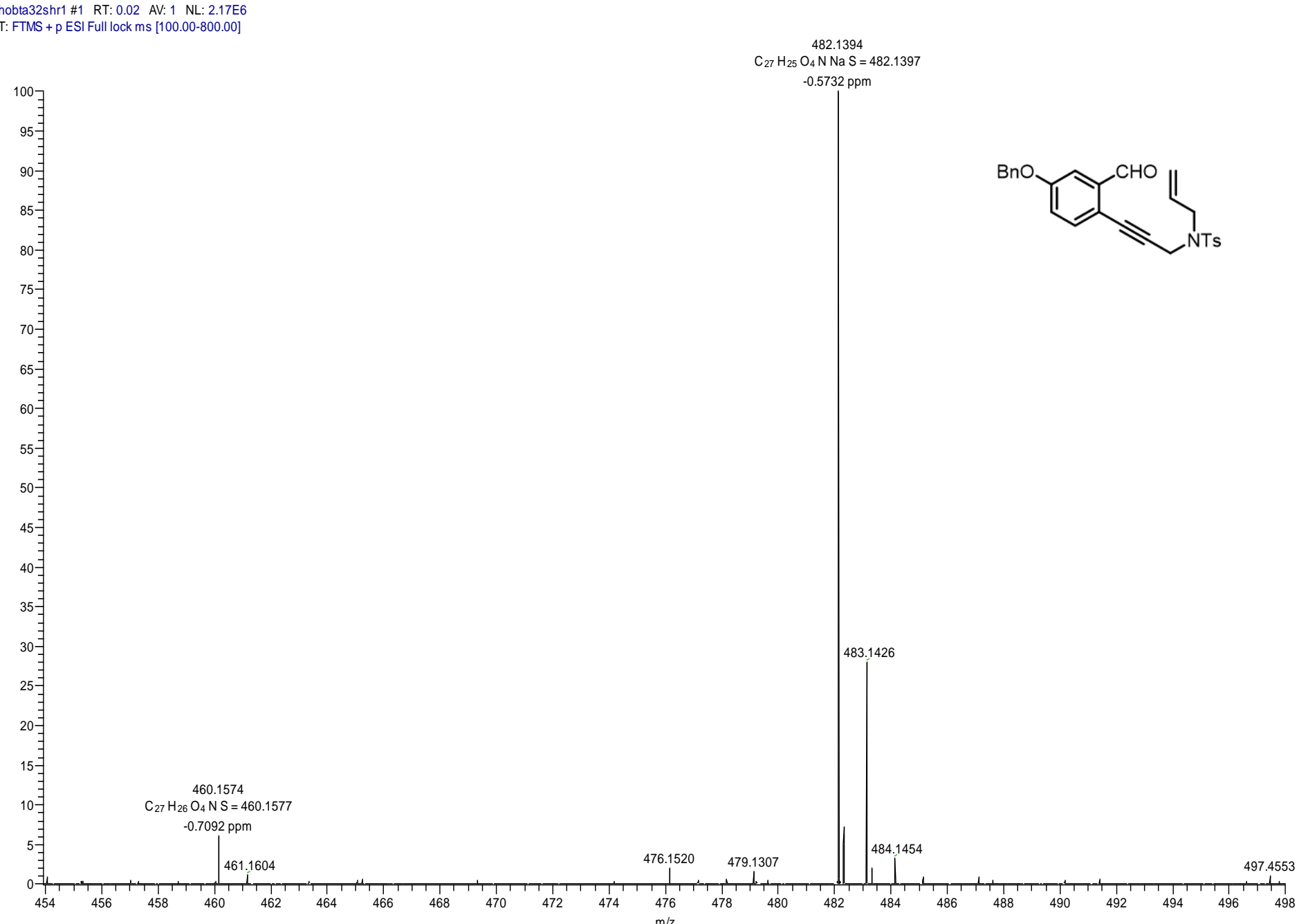

HRMS-ESI Compound 1J 

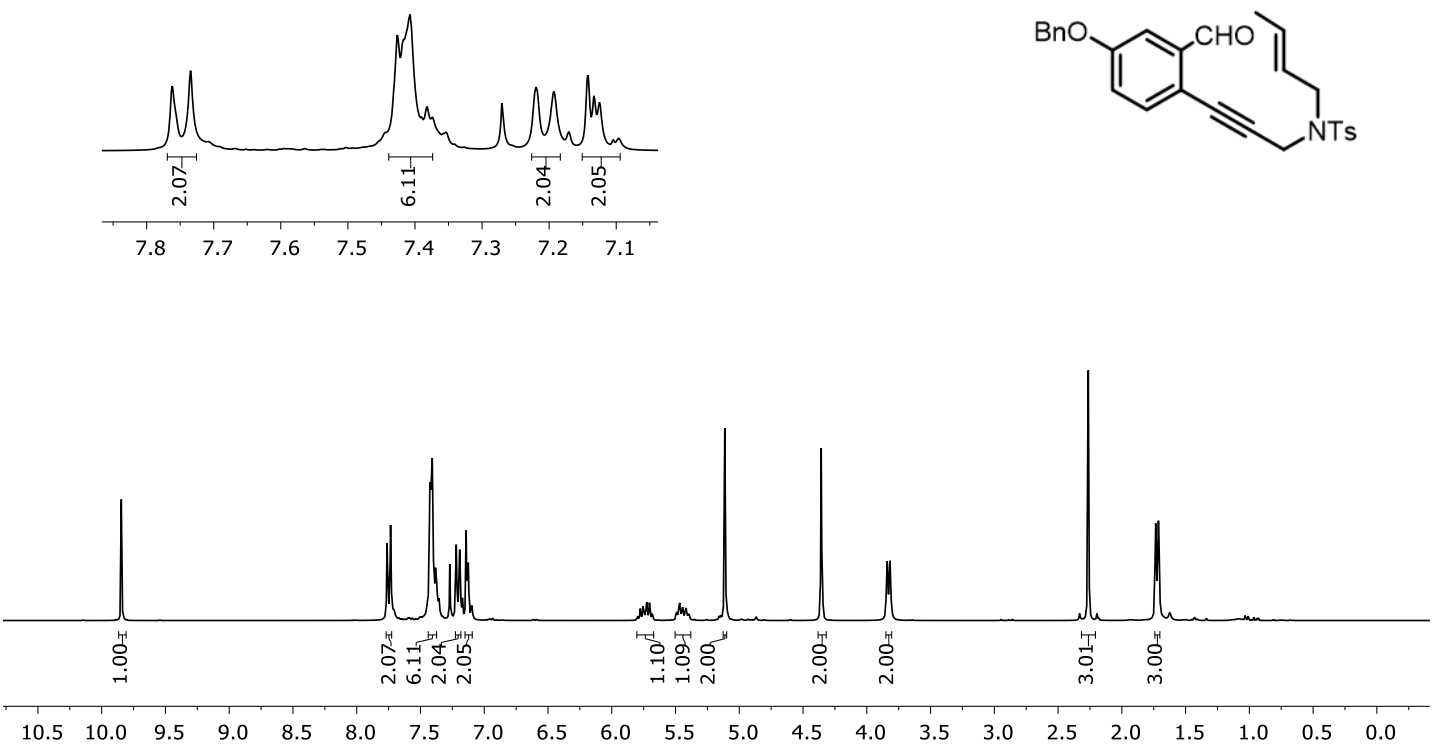

${ }^{1} \mathrm{H}$ NMR Compound $1 \mathbf{k}\left(300 \mathrm{MHz}, \mathrm{CDCl}_{3}\right)$
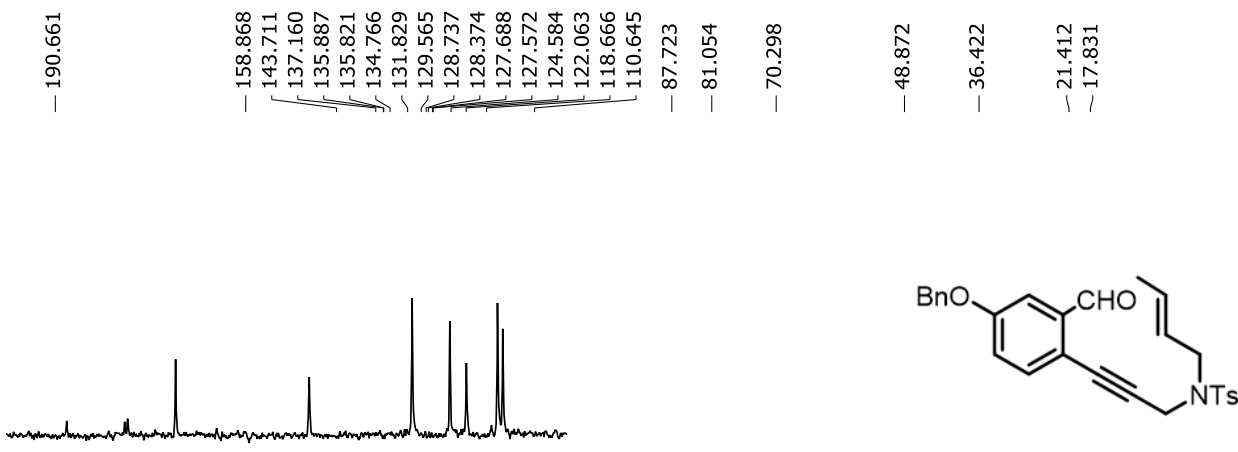

138137136135134133132131130129128127

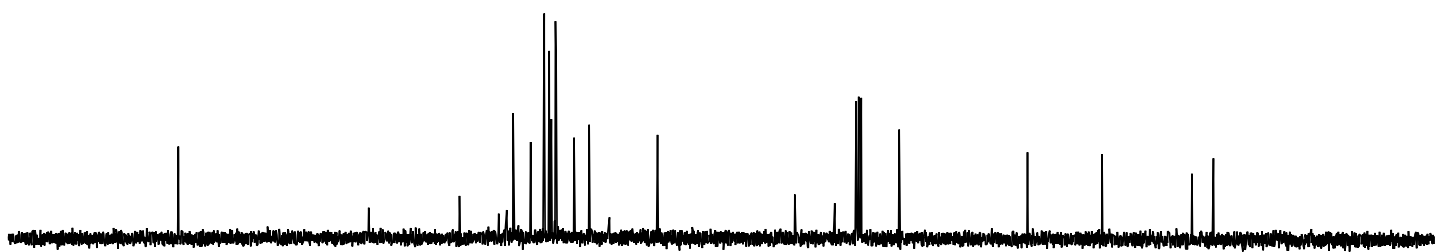

$\begin{array}{lllllllllllllllllllllll}210 & 200 & 190 & 180 & 170 & 160 & 150 & 140 & 130 & 120 & 110 & 100 & 90 & 80 & 70 & 60 & 50 & 40 & 30 & 20 & 10 & 0 & -10\end{array}$

${ }^{13} \mathrm{C}$ NMR Compound $1 \mathbf{k}\left(75 \mathrm{MHz}, \mathrm{CDCl}_{3}\right)$ 


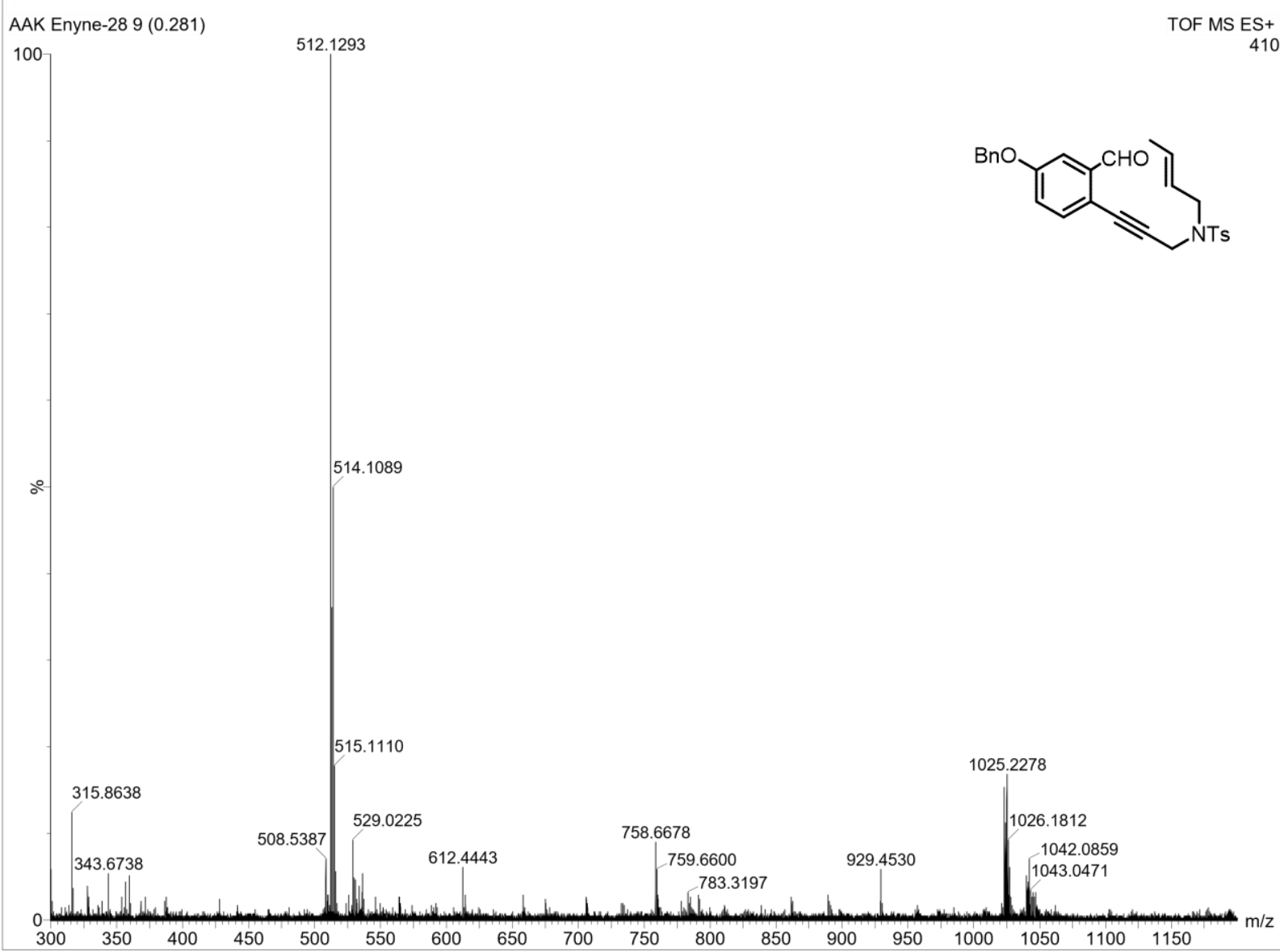

Mass-ESI Compound 1k 

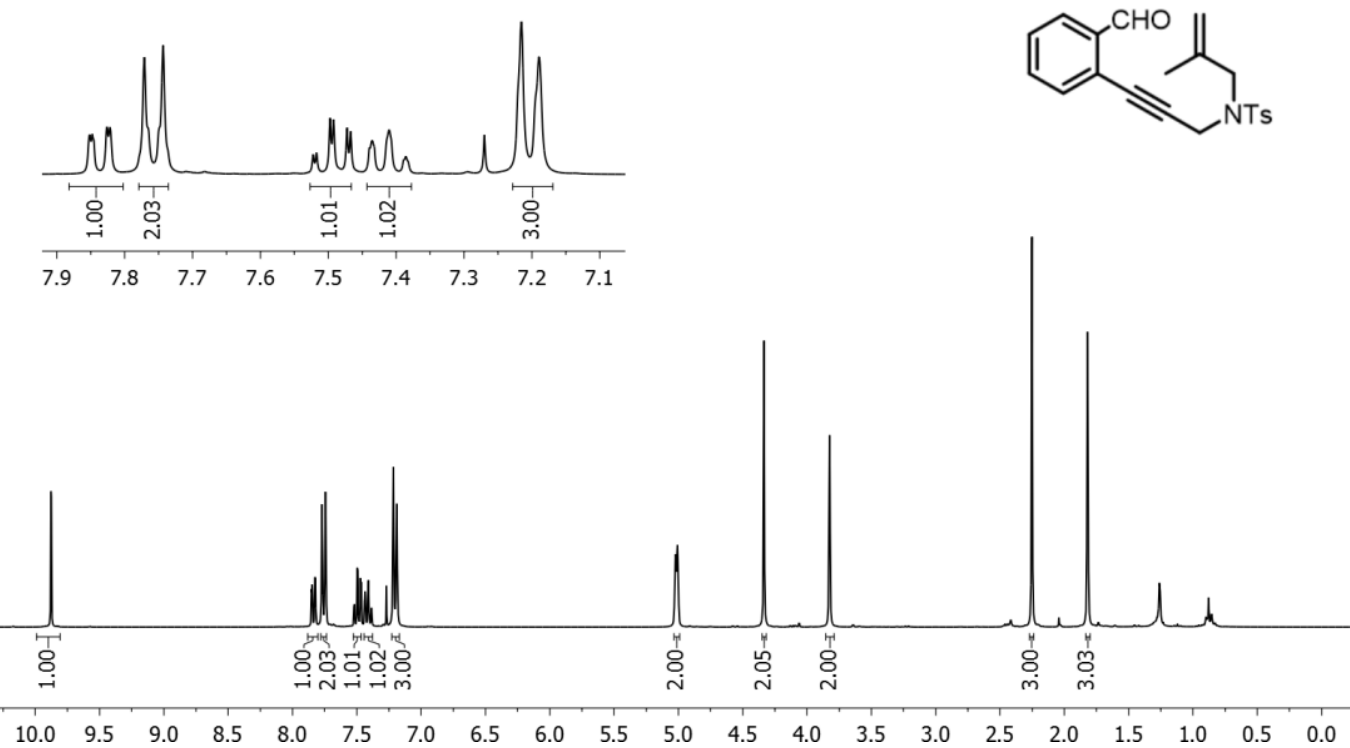

${ }^{1} \mathrm{H}$ NMR Compound 11 (300 MHz, $\mathrm{CDCl}_{3}$ )

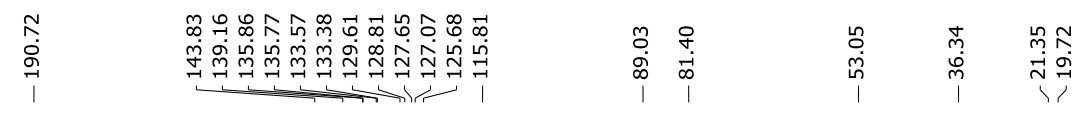
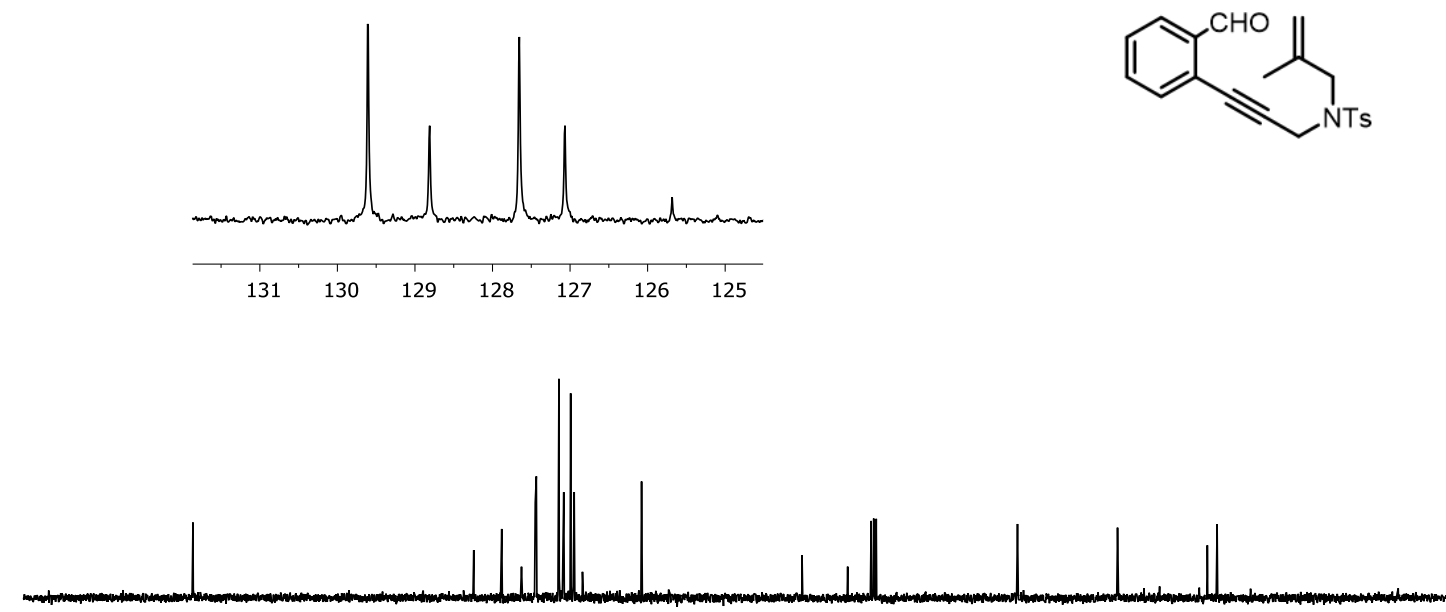

$\begin{array}{lllllllllllllllllllllll}210 & 200 & 190 & 180 & 170 & 160 & 150 & 140 & 130 & 120 & 110 & 100 & 90 & 80 & 70 & 60 & 50 & 40 & 30 & 20 & 10 & 0 & -10\end{array}$

${ }^{13} \mathrm{C}$ NMR Compound 11 ( $\left.75 \mathrm{MHz}, \mathrm{CDCl}_{3}\right)$ 
AAK Enyne-42 3 (0.070) Cm (3:28) TOF MS ES+

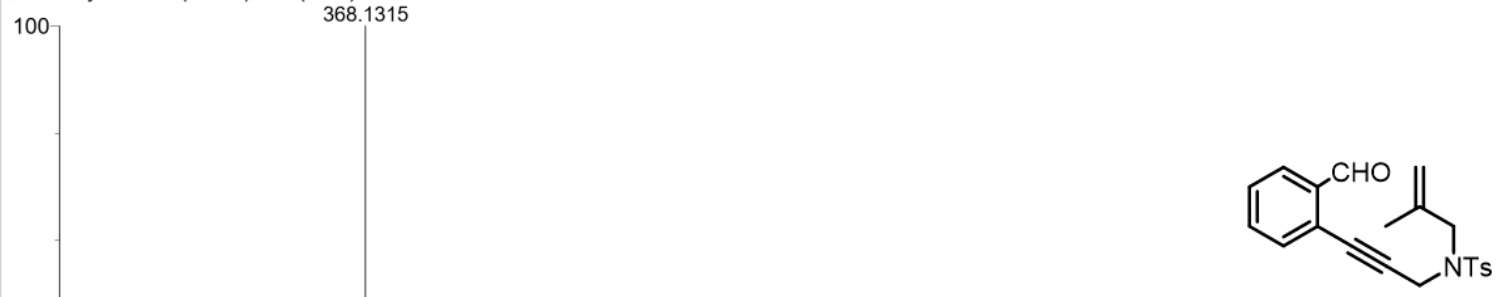

$3.81 \mathrm{e} 4$
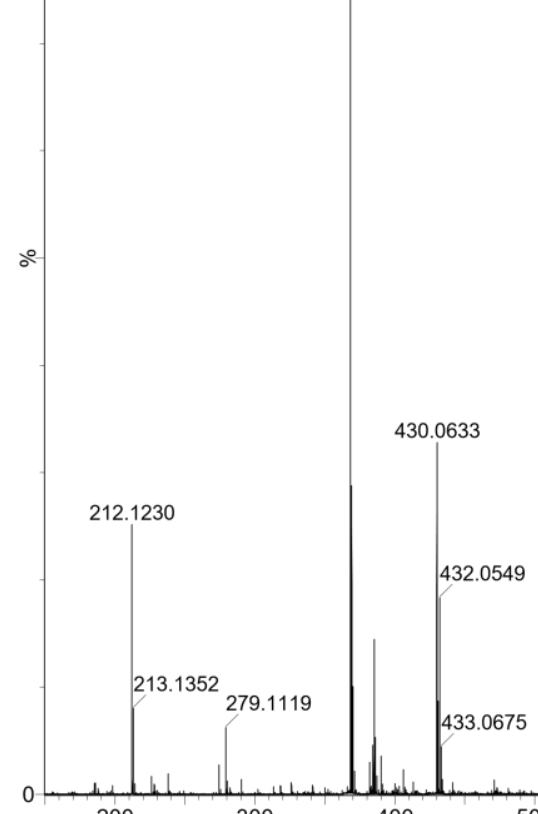

433.0675

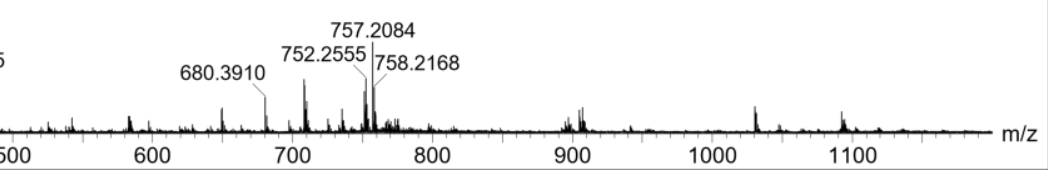

Mass-ESI Compound 11 


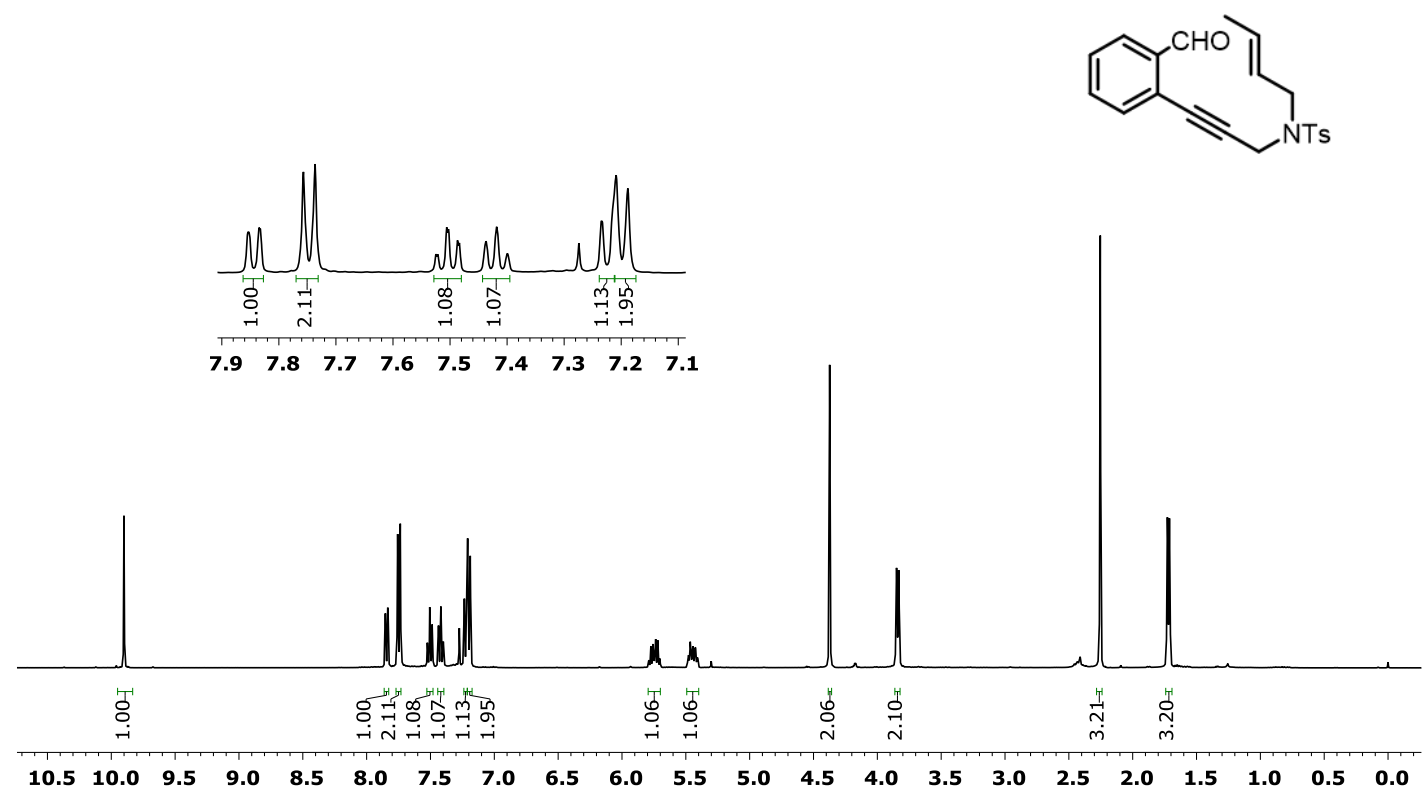

${ }^{1} \mathrm{H}$ NMR Compound $1 \mathrm{~m}\left(400 \mathrm{MHz}, \mathrm{CDCl}_{3}\right)$

\begin{tabular}{|c|c|c|c|c|c|}
\hline 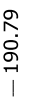 & 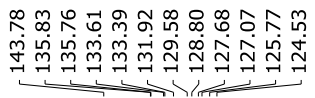 & $\begin{array}{c}\tilde{N} \\
\infty \\
\infty \\
1\end{array}$ & 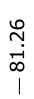 & $\begin{array}{c}m \\
\alpha \\
\infty \\
\infty \\
\mid\end{array}$ & $\begin{array}{c}\text { mे } \\
\text { iे } \\
\text { in }\end{array}$ \\
\hline
\end{tabular}
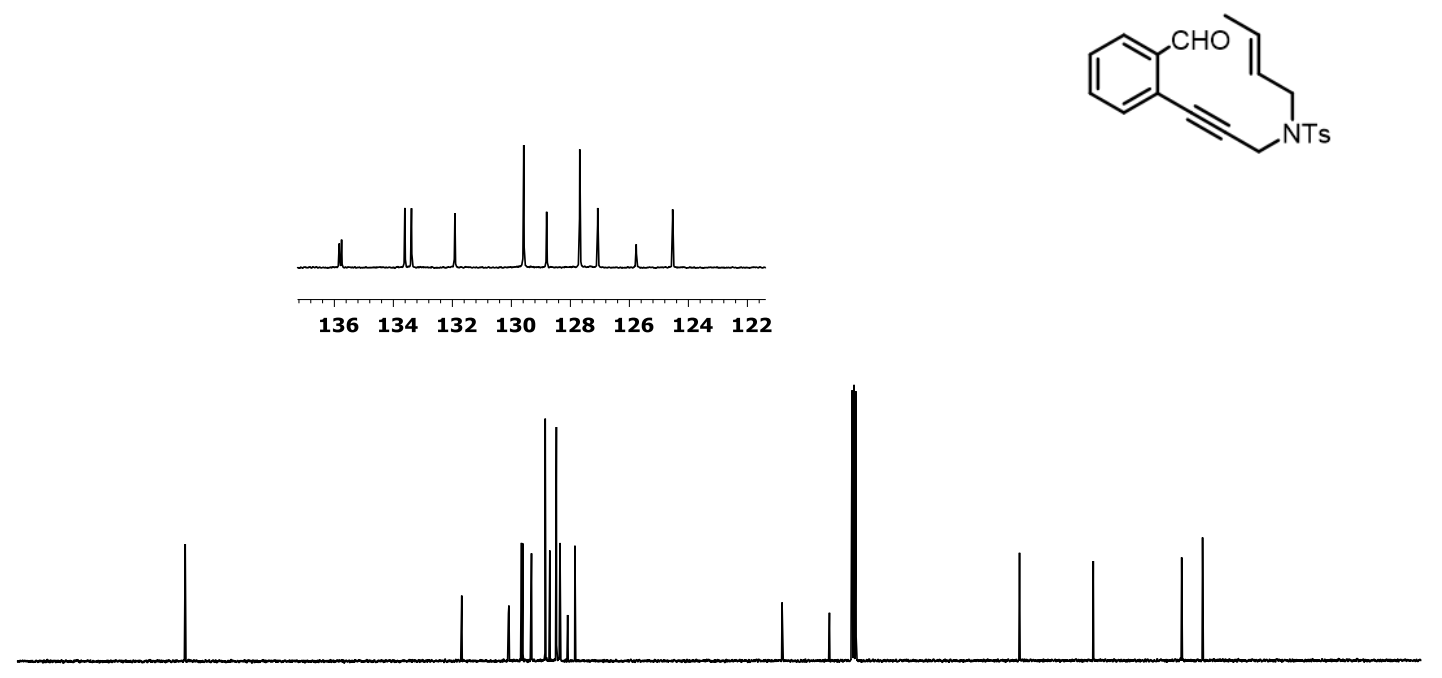

$\begin{array}{llllllllllllllllllllllllllllll}210 & 200 & 190 & 180 & 170 & 160 & 150 & 140 & 130 & 120 & 110 & 100 & 90 & 80 & 70 & 60 & 50 & 40 & 30 & 20 & 10 & 0 & -10\end{array}$

${ }^{13} \mathrm{C}$ NMR Compound $1 \mathrm{~m}\left(100 \mathrm{MHz}, \mathrm{CDCl}_{3}\right)$ 


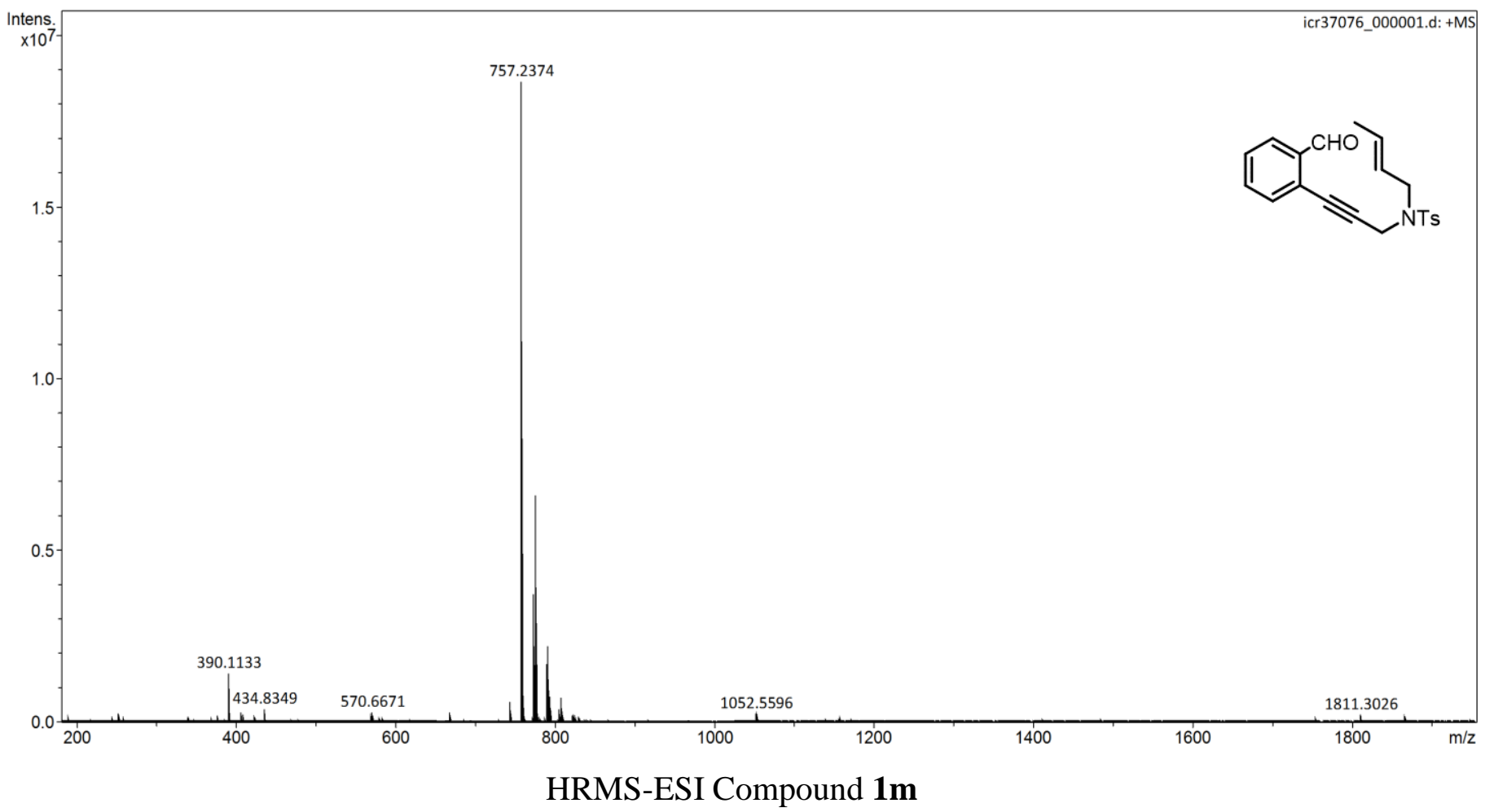

\begin{tabular}{|c|c|c|c|c|c|c|c|}
\hline Meas. m/z & Ion Formula & $\mathrm{m} / \mathrm{z}$ & err $[\mathrm{ppm}]$ & mSigma & $\mathrm{rdb}$ & $\mathrm{e}^{-}$Conf & N-Rule \\
\hline \multirow[t]{2}{*}{368.1313} & $\mathrm{C} 27 \mathrm{H} 16 \mathrm{~N} 2$ & 368.1308 & -1.5 & 22.9 & 21.0 & odd & ok \\
\hline & $\mathrm{C} 21 \mathrm{H} 22 \mathrm{NO} 3 \mathrm{~S}$ & 368.1315 & 0.4 & 37.2 & 11.5 & even & ok \\
\hline \multirow[t]{4}{*}{390.1133} & $\mathrm{C} 20 \mathrm{H} 22 \mathrm{O} 6 \mathrm{~S}$ & 390.1132 & -0.3 & 9.1 & 10.0 & odd & ok \\
\hline & $\mathrm{C} 21 \mathrm{H} 21 \mathrm{NNaO} 3 \mathrm{~S}$ & 390.1134 & 0.4 & 10.3 & 11.5 & even & ok \\
\hline & C26H16NO3 & 390.1125 & -2.0 & 37.6 & 19.5 & even & ok \\
\hline & $\mathrm{C} 27 \mathrm{H} 15 \mathrm{~N} 2 \mathrm{Na}$ & 390.1127 & -1.3 & 42.7 & 21.0 & odd & ok \\
\hline \multirow[t]{3}{*}{757.2374} & $\mathrm{C} 42 \mathrm{H} 34 \mathrm{~N} 6 \mathrm{NaO} 7$ & 757.2381 & 0.9 & 18.2 & 28.5 & even & ok \\
\hline & C42H39N5O5S2 & 757.2387 & 1.7 & 18.5 & 26.0 & odd & ok \\
\hline & $\mathrm{C} 42 \mathrm{H} 42 \mathrm{~N} 2 \mathrm{NaO} 6 \mathrm{~S} 2$ & 757.2376 & 0.3 & 18.8 & 22.5 & even & ok \\
\hline
\end{tabular}



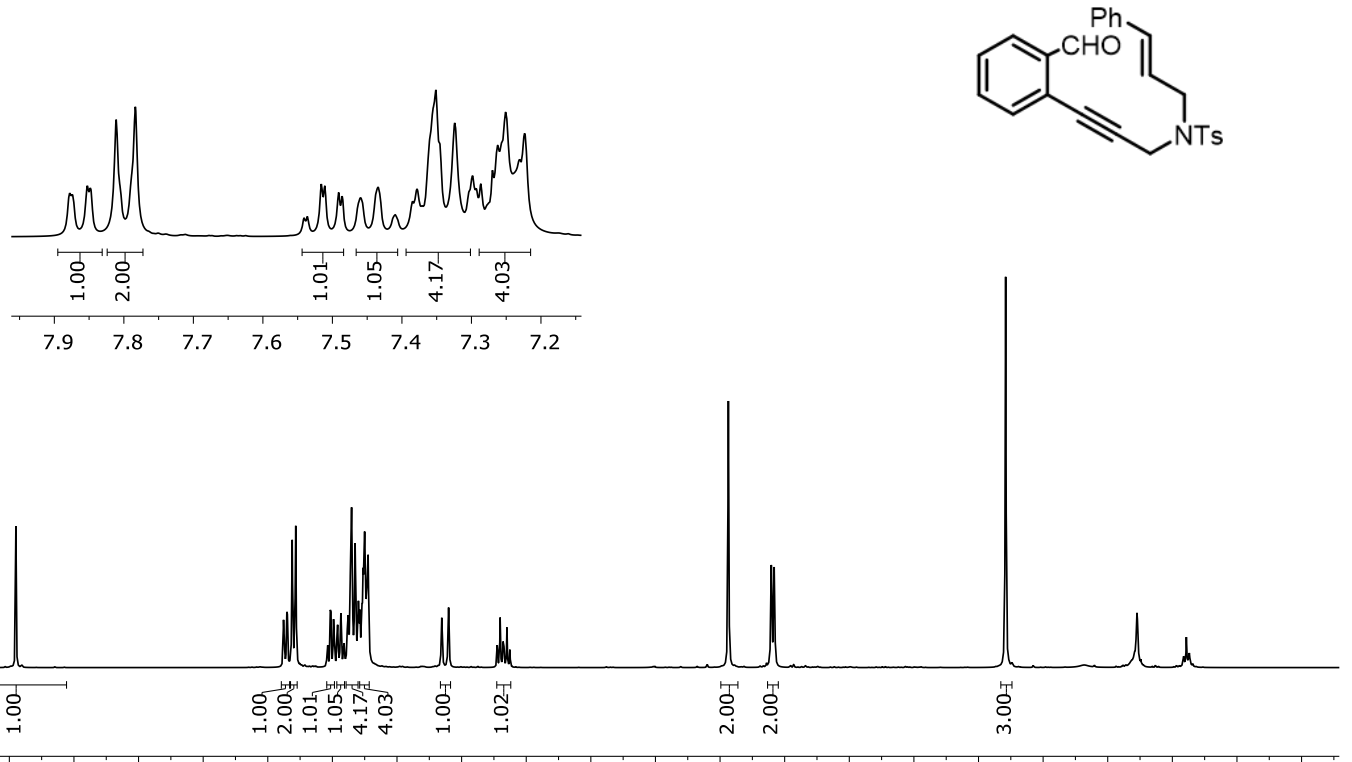

$\begin{array}{llllllllllllllllllllll}10.5 & 10.0 & 9.5 & 9.0 & 8.5 & 8.0 & 7.5 & 7.0 & 6.5 & 6.0 & 5.5 & 5.0 & 4.5 & 4.0 & 3.5 & 3.0 & 2.5 & 2.0 & 1.5 & 1.0 & 0.5 & 0.0\end{array}$

${ }^{1} \mathrm{H}$ NMR Compound 1 (n $\left(300 \mathrm{MHz}, \mathrm{CDCl}_{3}\right)$

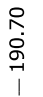
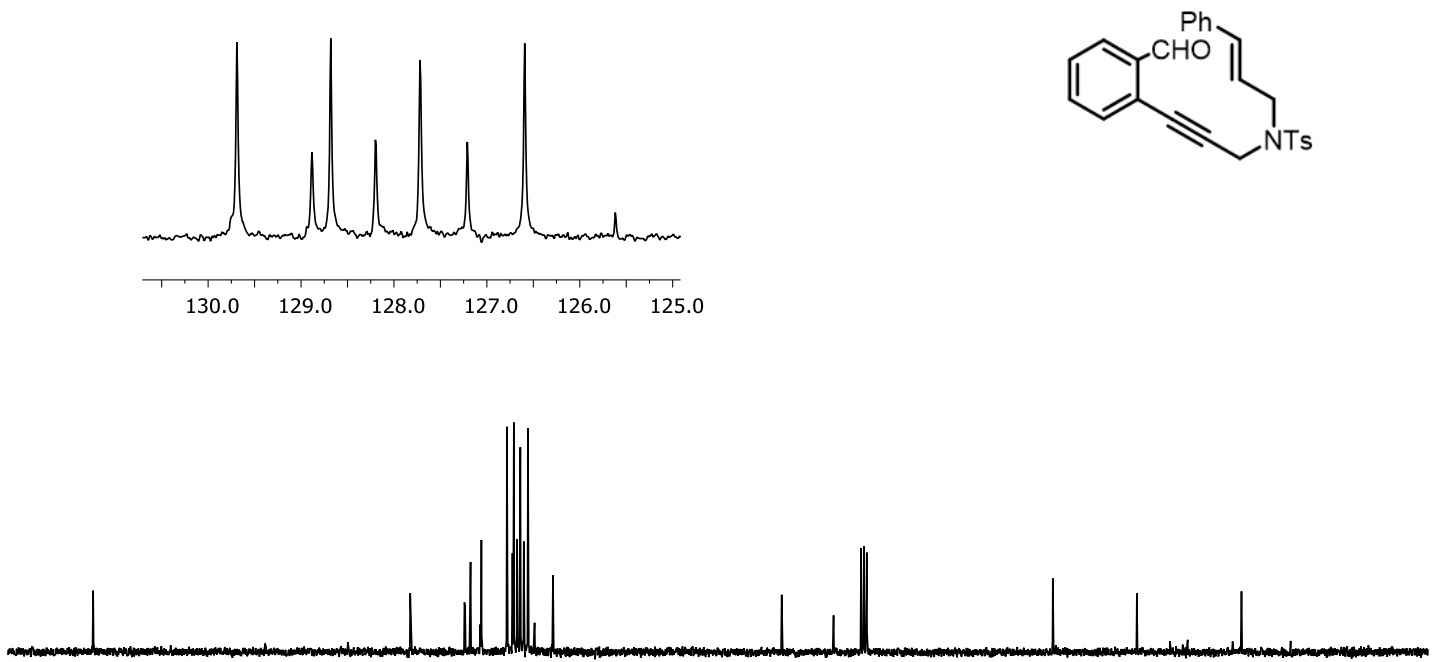

$\begin{array}{lllllllllllllllllllll}200 & 190 & 180 & 170 & 160 & 150 & 140 & 130 & 120 & 110 & 100 & 90 & 80 & 70 & 60 & 50 & 40 & 30 & 20 & 10 & 0\end{array}$

${ }^{13} \mathrm{C}$ NMR Compound $1 n\left(75 \mathrm{MHz}, \mathrm{CDCl}_{3}\right)$ 
AAK Enyne-44 4 (0.106) Cm (4:42)

TOF MS ES+

100 $1.49 \mathrm{e} 4$

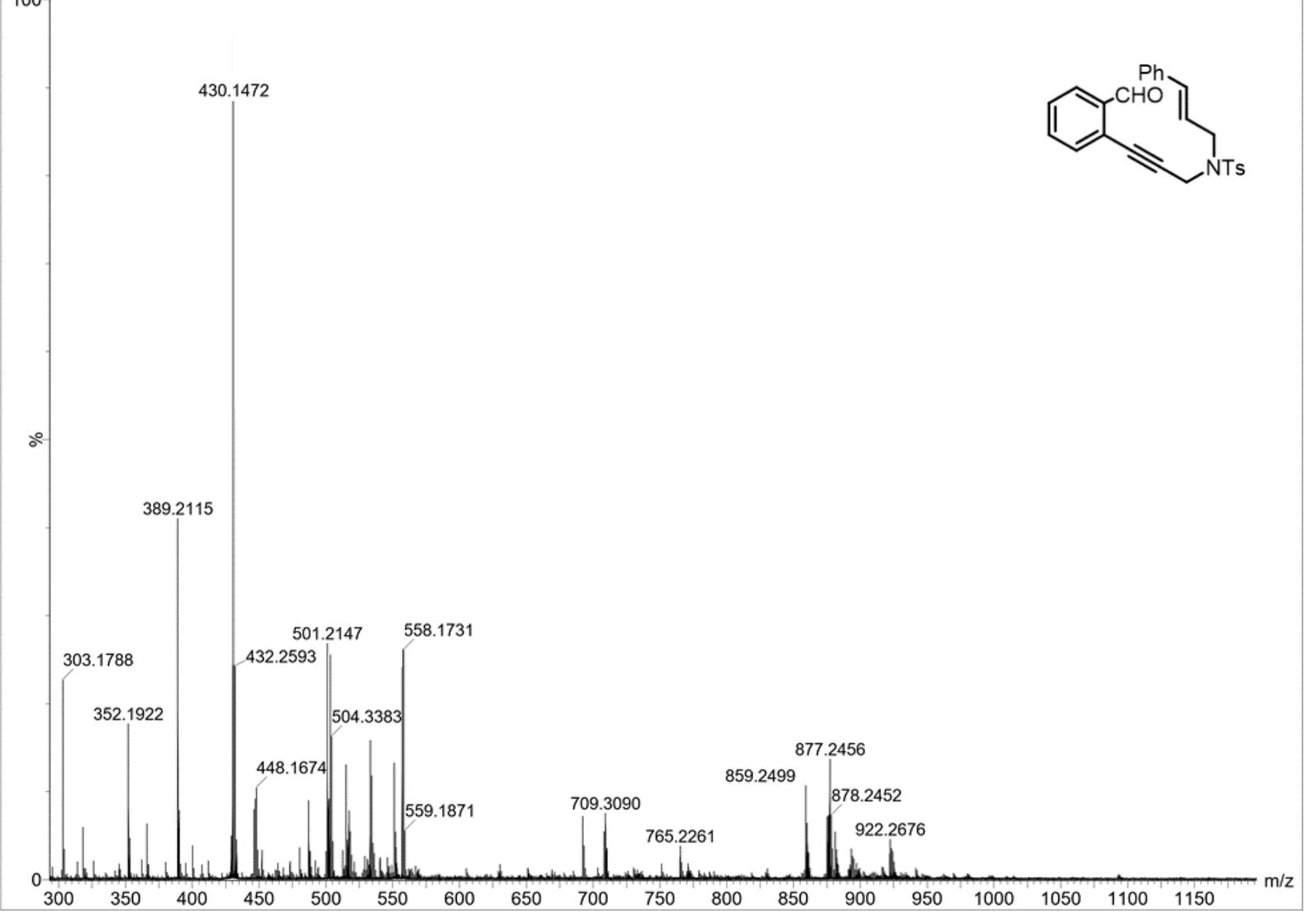

Mass-ESI Compound 1n 

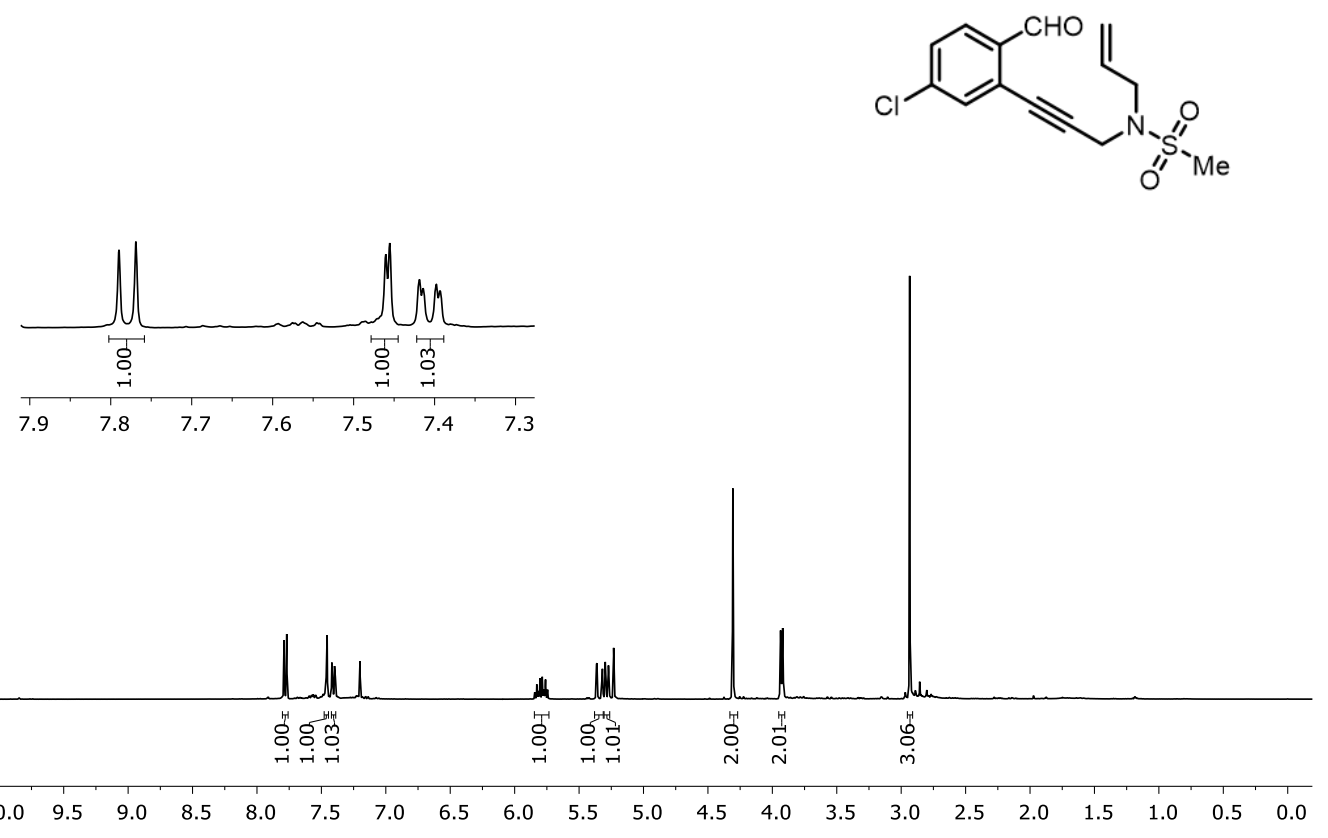

${ }^{1} \mathrm{H}$ NMR Compound $10\left(400 \mathrm{MHz}, \mathrm{CDCl}_{3}\right)$

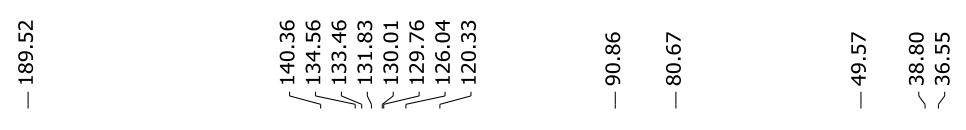
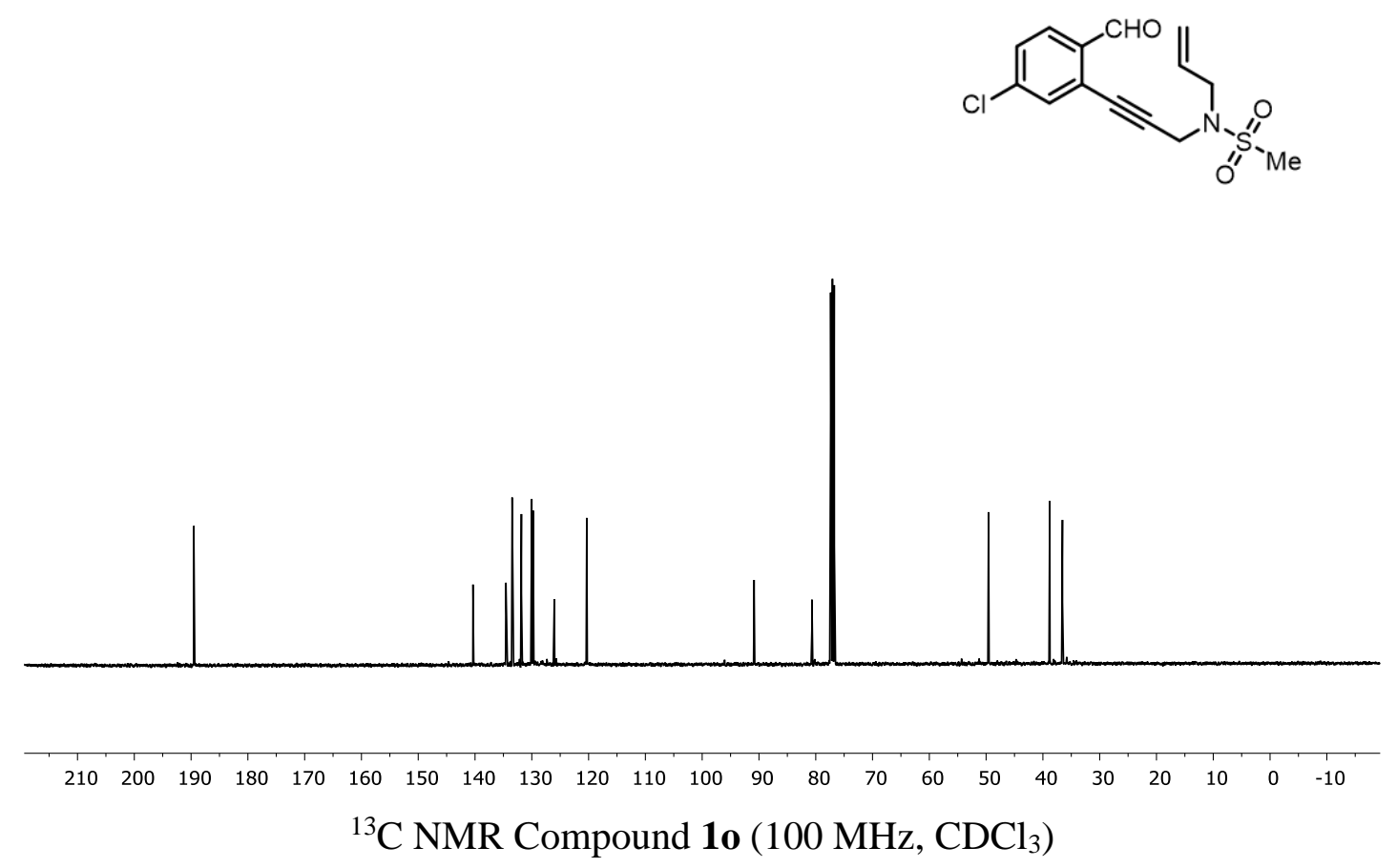


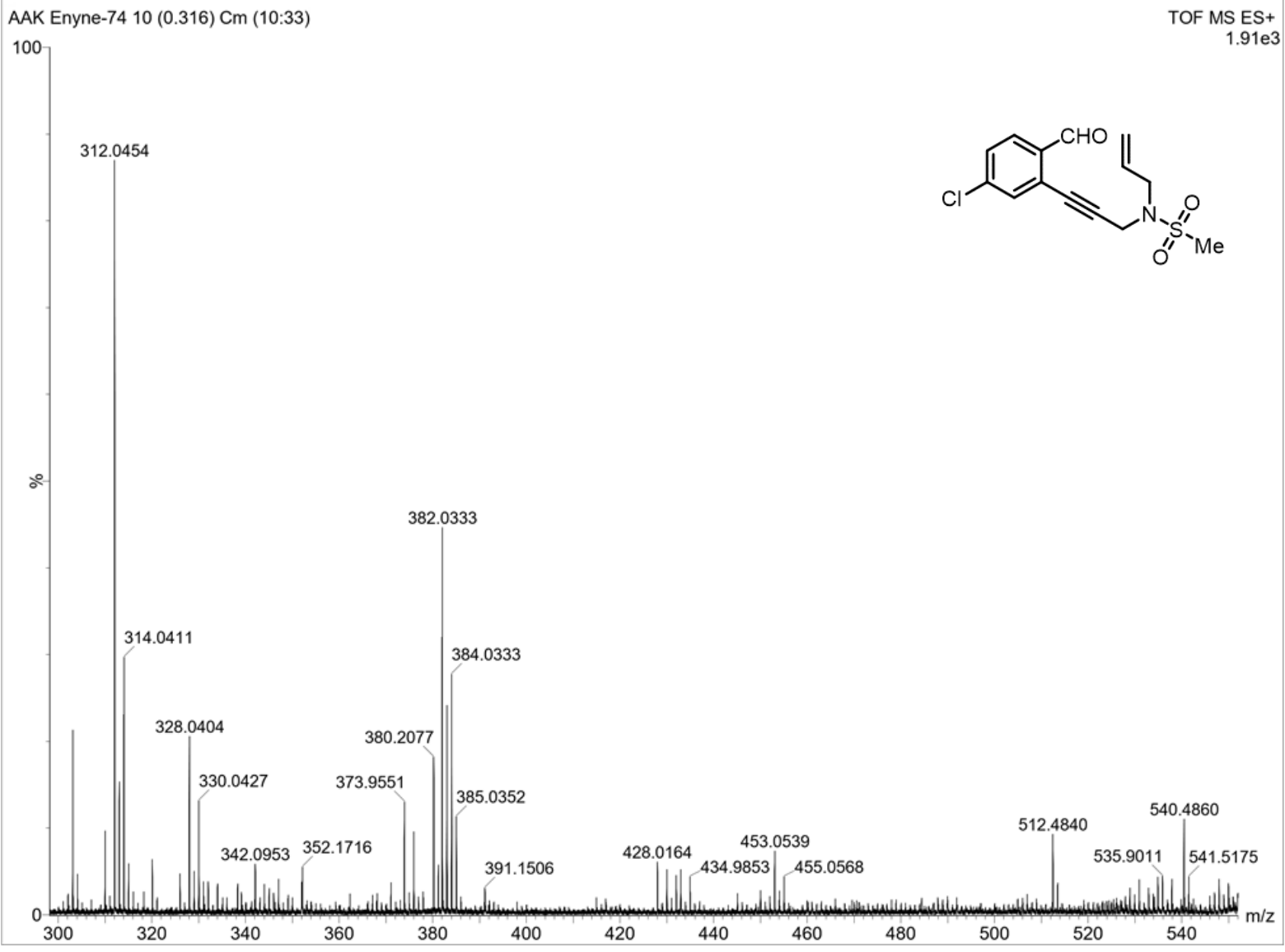

Mass-ESI Compound 10 

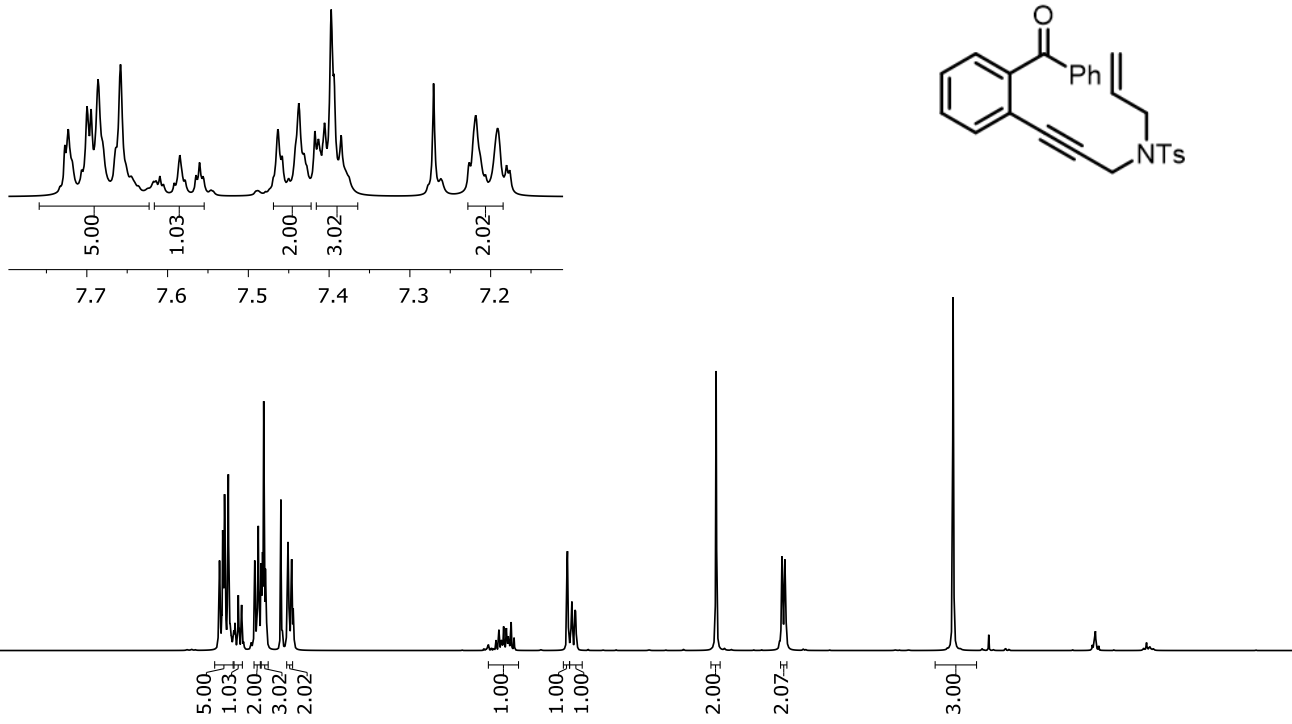

${ }^{1} \mathrm{H}$ NMR Compound 3a $\left(300 \mathrm{MHz}, \mathrm{CDCl}_{3}\right)$

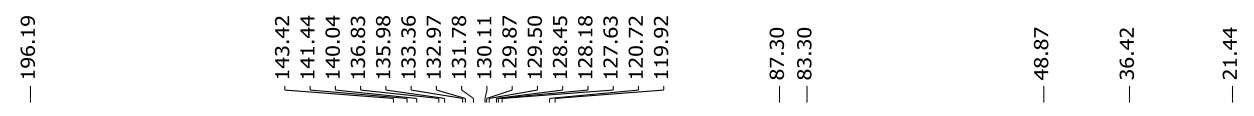
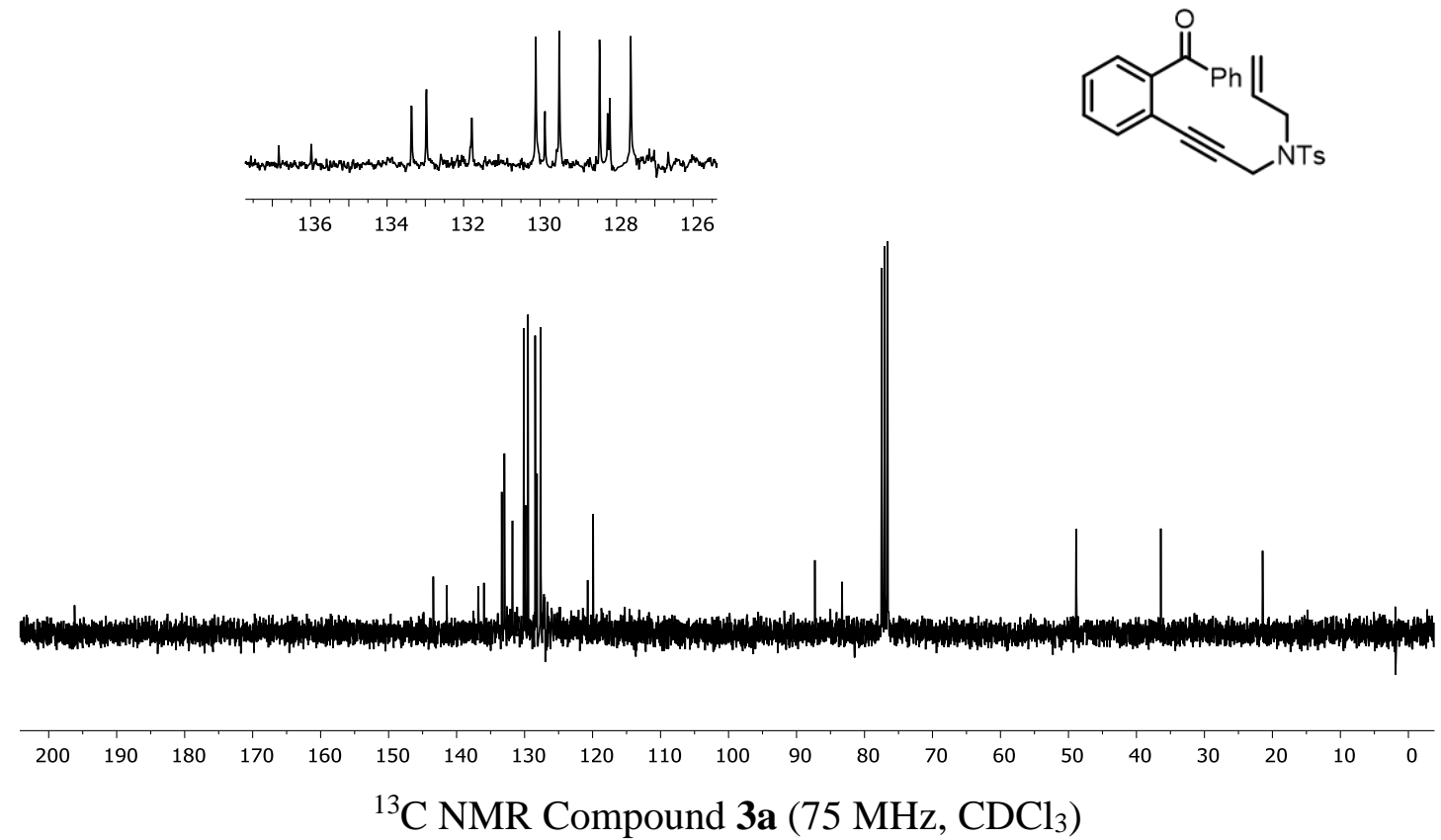
AAK Enyne-38 12 (0.421)

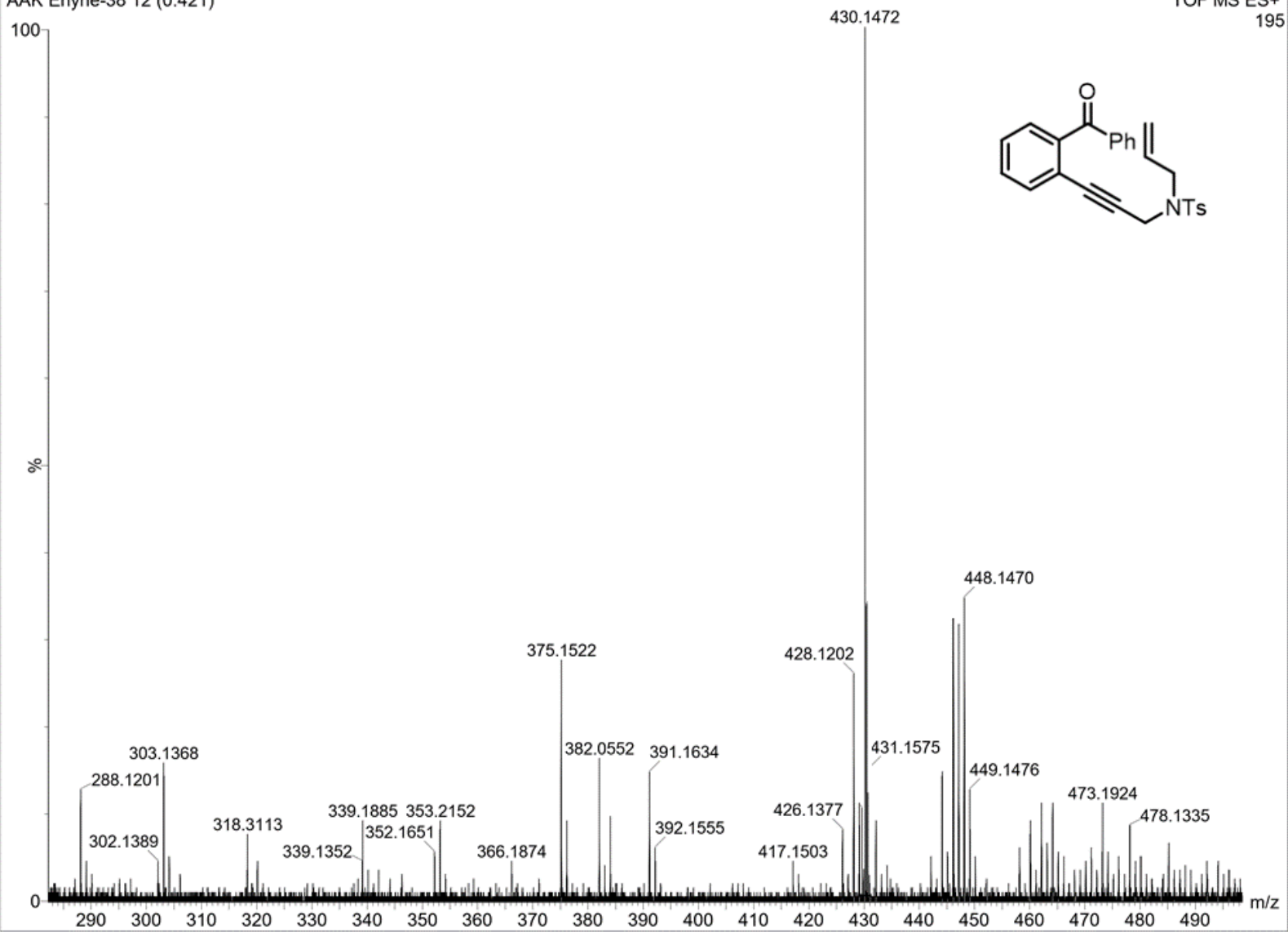

Mass-ESI Compound 3a 

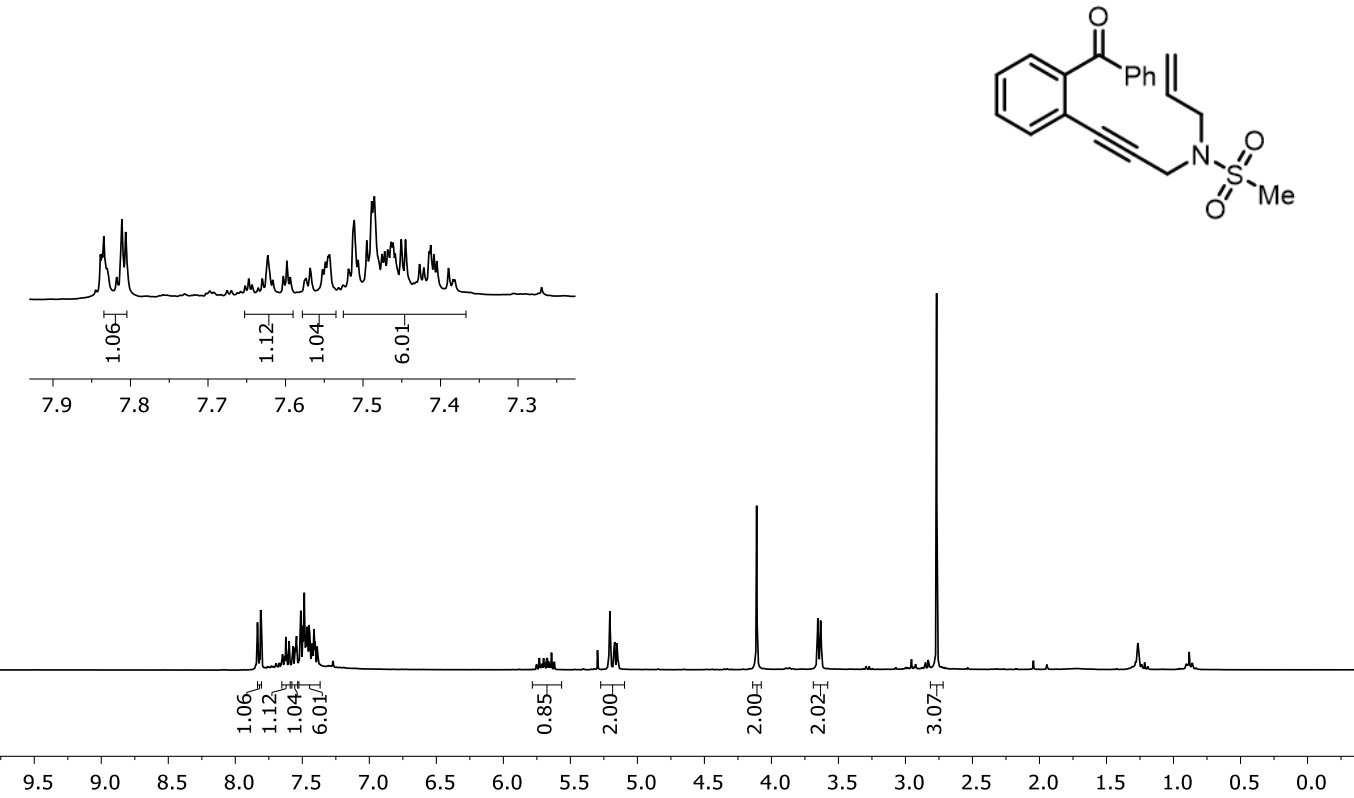

${ }^{1} \mathrm{H}$ NMR Compound $\mathbf{3 b}\left(300 \mathrm{MHz}, \mathrm{CDCl}_{3}\right)$

\begin{tabular}{l}
$\stackrel{5}{m}$ \\
$\stackrel{\circ}{\circ}$ \\
\multirow{1}{1}{}
\end{tabular}

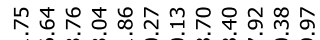
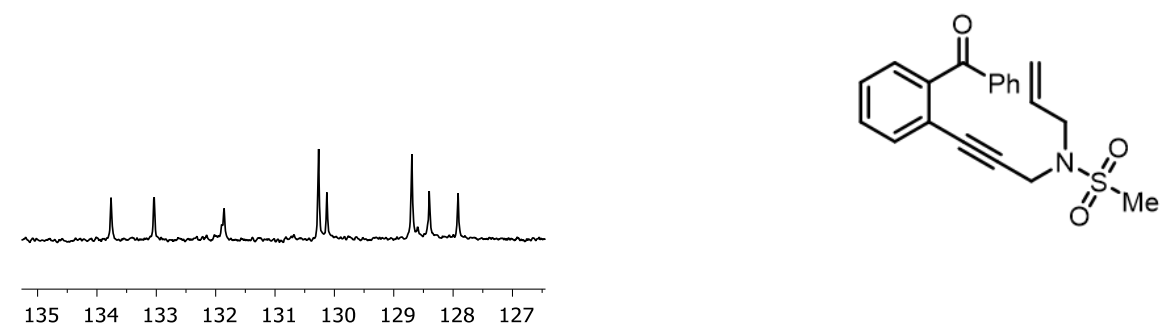

$\begin{array}{lllllllll}135 & 134 & 133 & 132 & 131 & 130 & 129 & 128 & 127\end{array}$

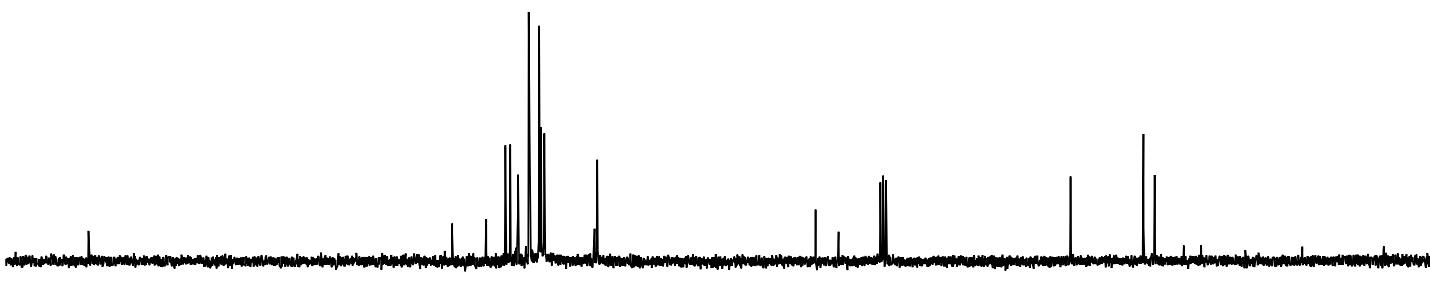

$\begin{array}{lllllllllllllllllllll}200 & 190 & 180 & 170 & 160 & 150 & 140 & 130 & 120 & 110 & 100 & 90 & 80 & 70 & 60 & 50 & 40 & 30 & 20 & 10 & 0\end{array}$

${ }^{13} \mathrm{C}$ NMR Compound $\mathbf{3 b}\left(75 \mathrm{MHz}, \mathrm{CDCl}_{3}\right)$ 
AAK Enyne-52 4 (0.106) Cm (4:58)

100

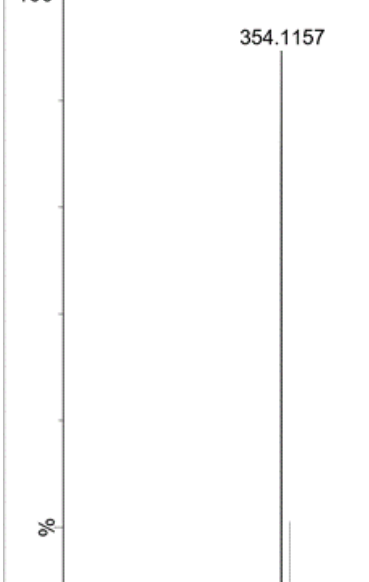

TOF MS ES+

$1.78 \mathrm{e} 4$

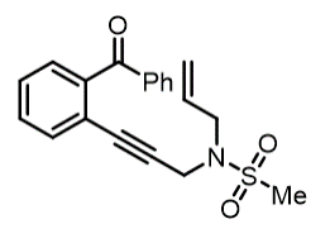

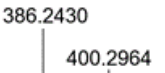

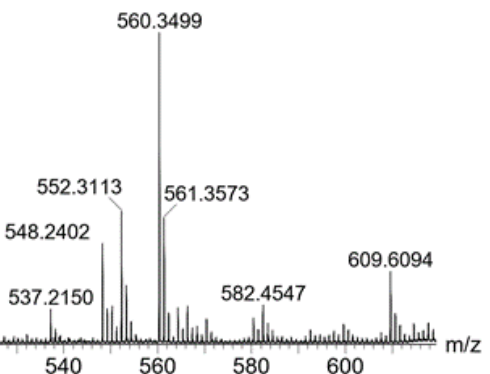

Mass-ESI Compound 3b 

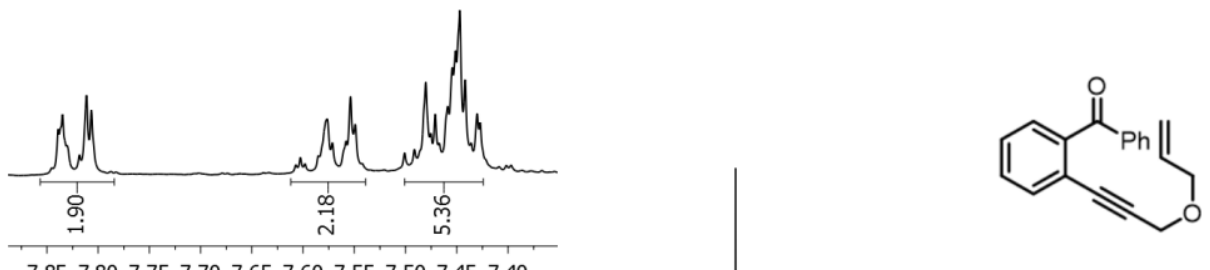

7.857 .807 .757 .707 .657 .607 .557 .507 .457 .40

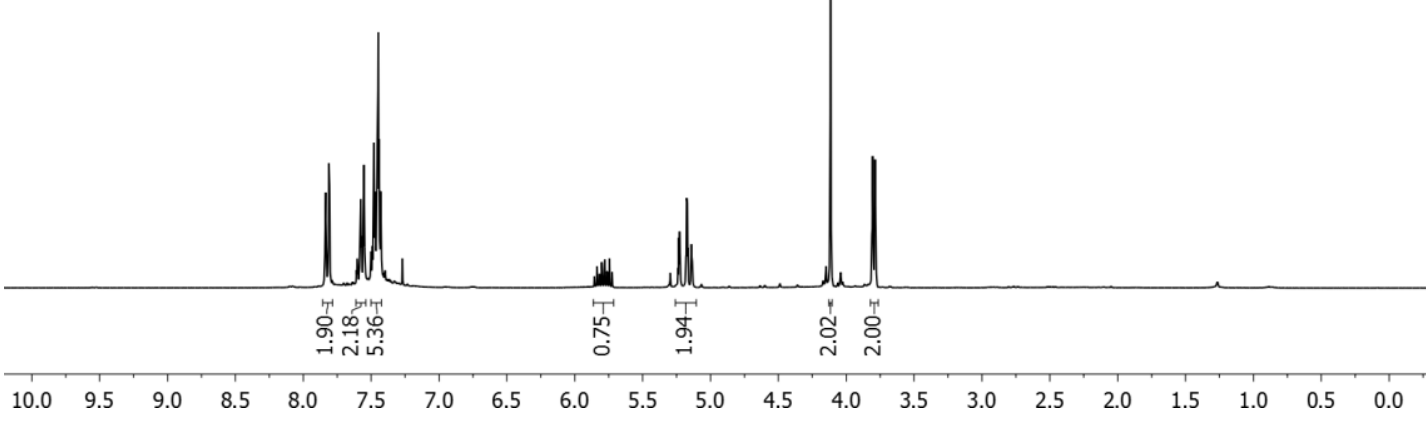

${ }^{1} \mathrm{H}$ NMR Compound 3c (300 MHz, $\left.\mathrm{CDCl}_{3}\right)$
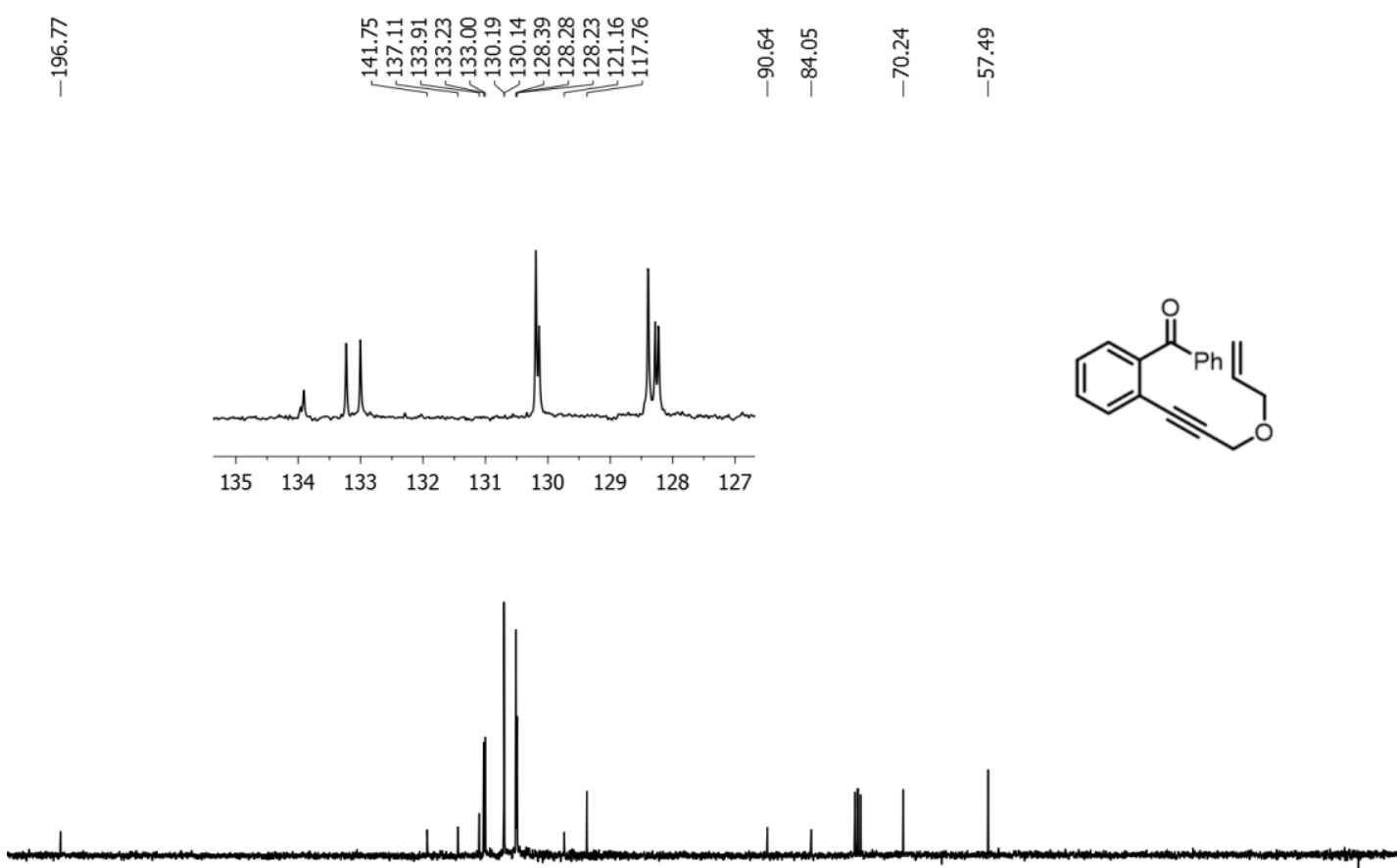

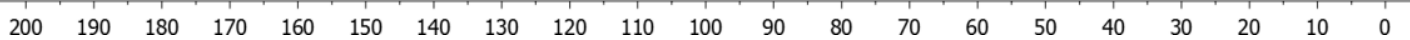

${ }^{13} \mathrm{CNMR}$ Compound $3 \mathbf{c}\left(75 \mathrm{MHz}, \mathrm{CDCl}_{3}\right)$ 


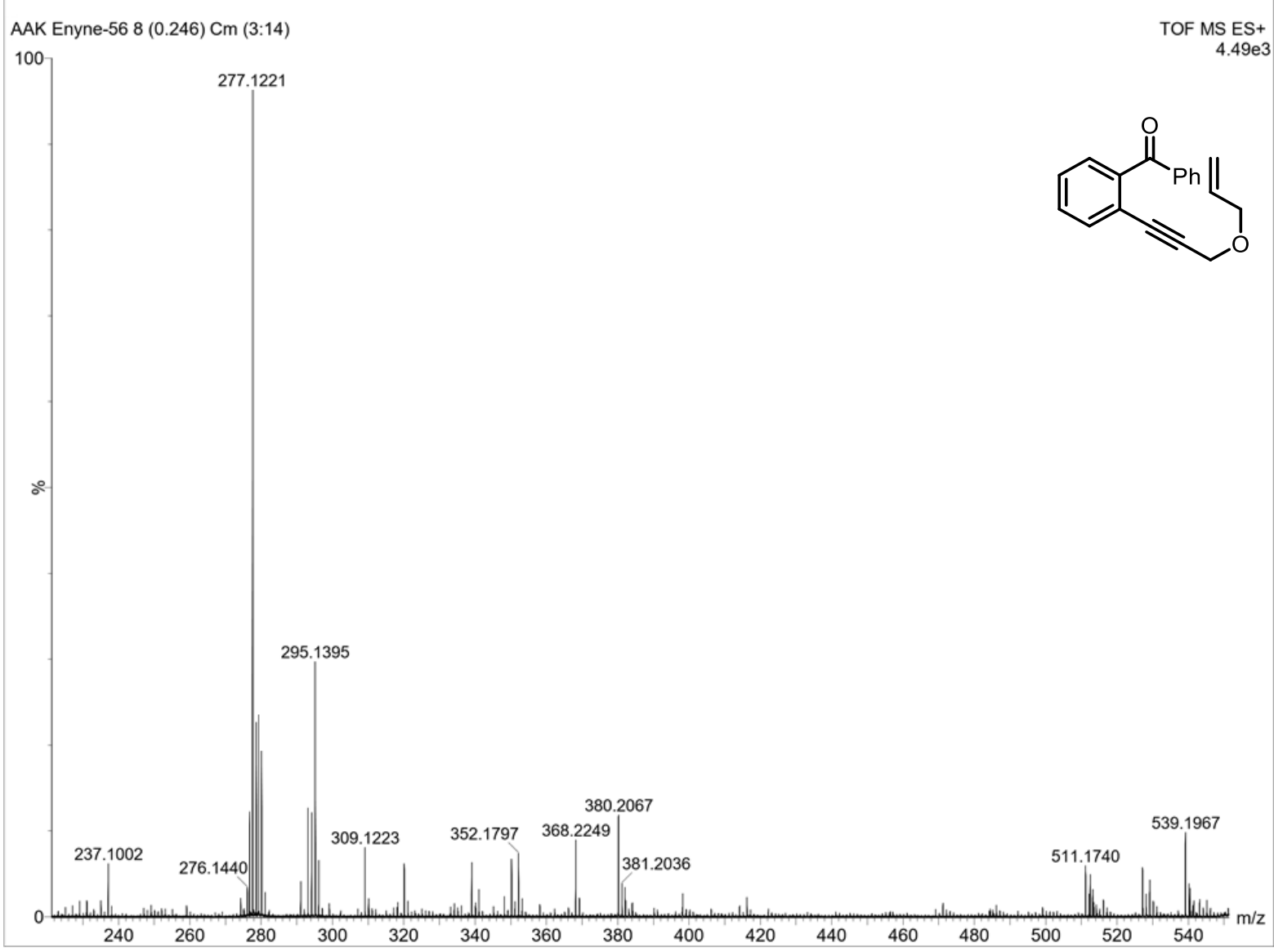

Mass-ESI Compound 3c 

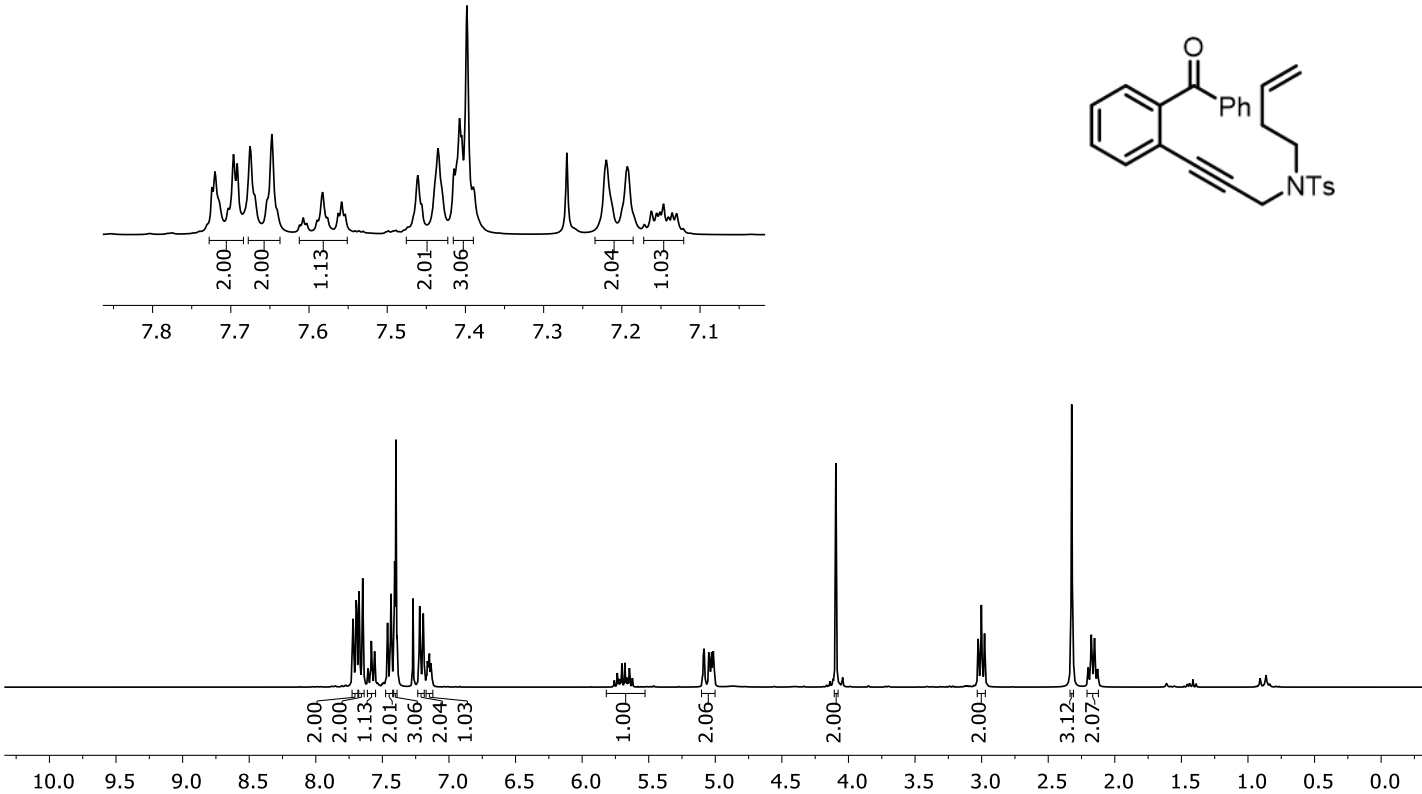

${ }^{1} \mathrm{H}$ NMR Compound 3d $\left(300 \mathrm{MHz}, \mathrm{CDCl}_{3}\right)$

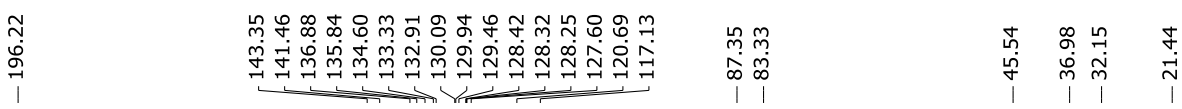
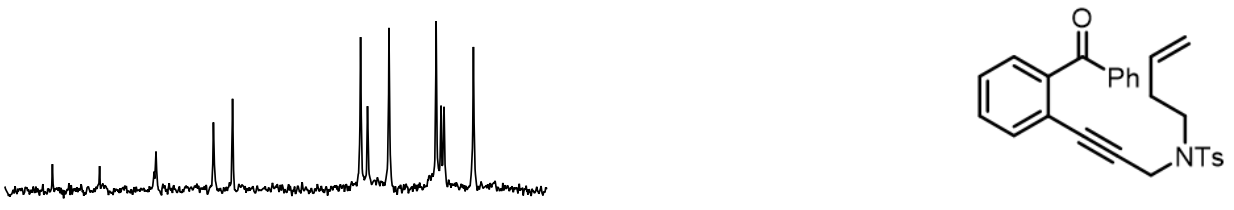

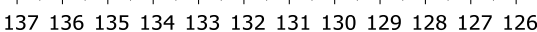

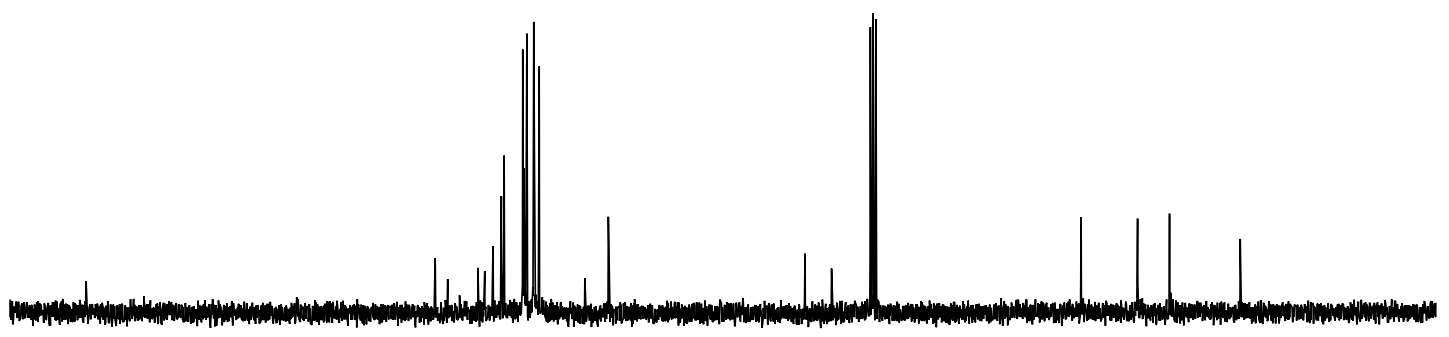

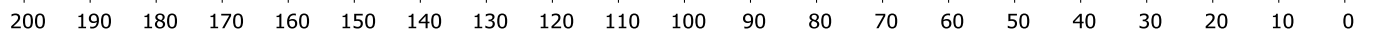

${ }^{13} \mathrm{C}$ NMR Compound 3d $\left(75 \mathrm{MHz}, \mathrm{CDCl}_{3}\right)$ 
AAK Enyne-64 5 (0.140) Cm (3:53)

100

TOF MS ES+
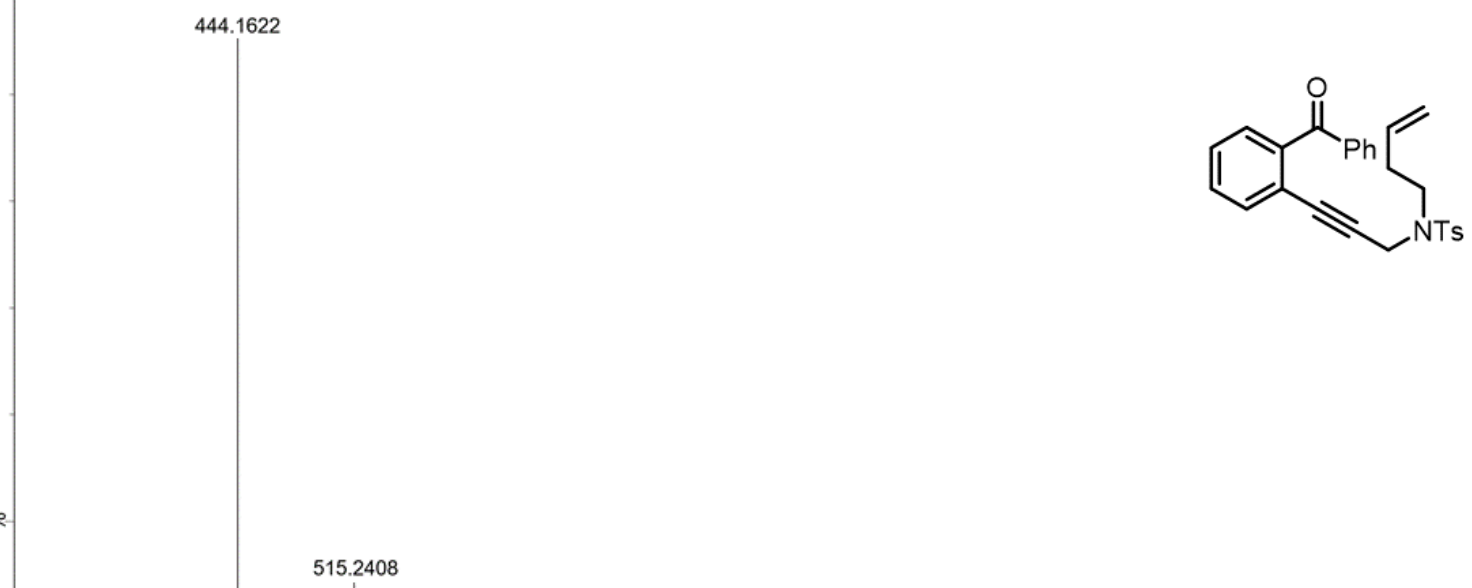

61.1761

557.1808

|||||| 558.1825

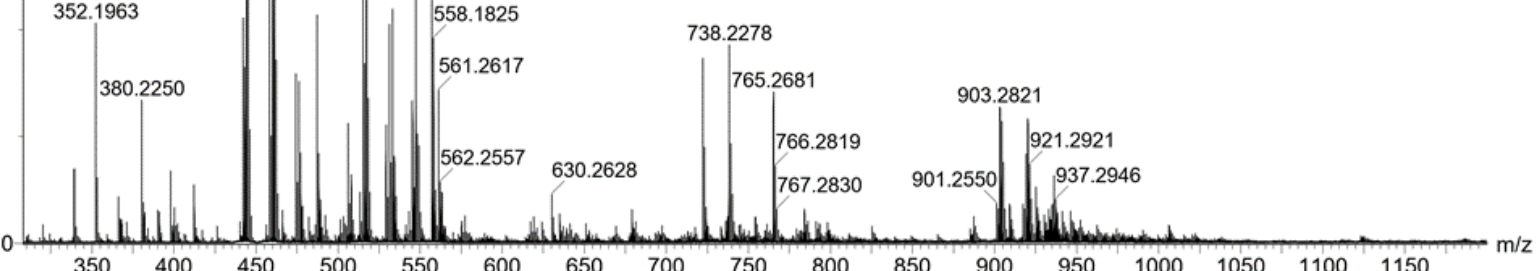

Mass-ESI Compound 3d 

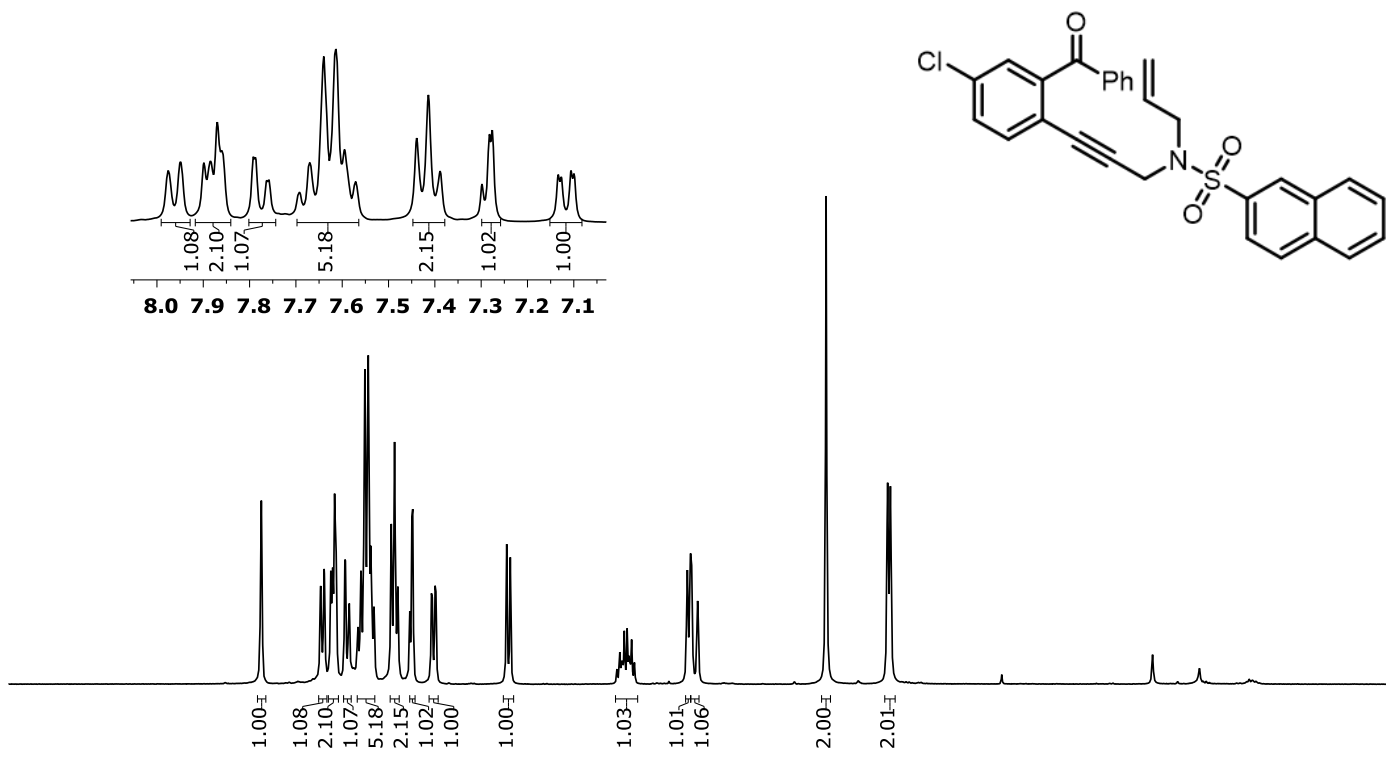

$\begin{array}{lllllllllllllllllllll}10.0 & 9.5 & 9.0 & 8.5 & 8.0 & 7.5 & 7.0 & 6.5 & 6.0 & 5.5 & 5.0 & 4.5 & 4.0 & 3.5 & 3.0 & 2.5 & 2.0 & 1.5 & 1.0 & 0.5 & 0.0\end{array}$

${ }^{1} \mathrm{H}$ NMR Compound $3 e\left(300 \mathrm{MHz}, \mathrm{CDCl}_{3}\right)$

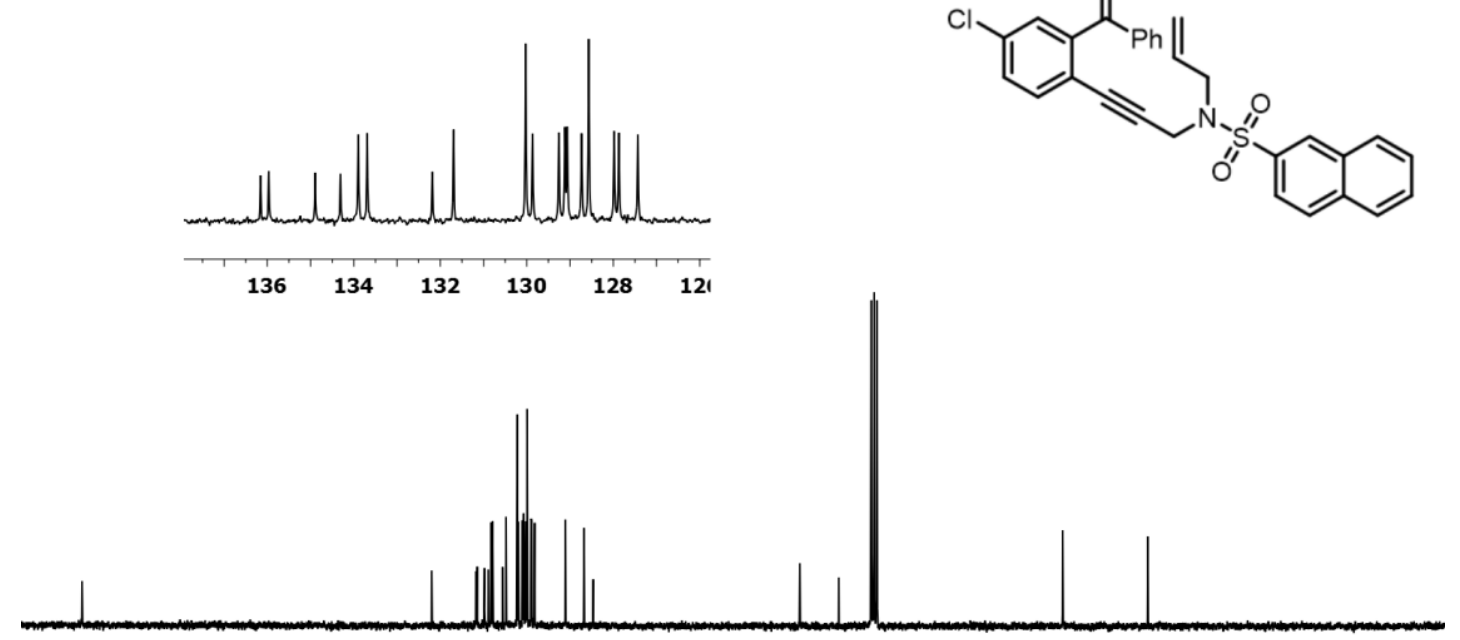

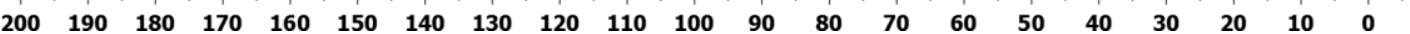

${ }^{13} \mathrm{C}$ NMR Compound $3 \mathbf{e}\left(75 \mathrm{MHz}, \mathrm{CDCl}_{3}\right)$ 


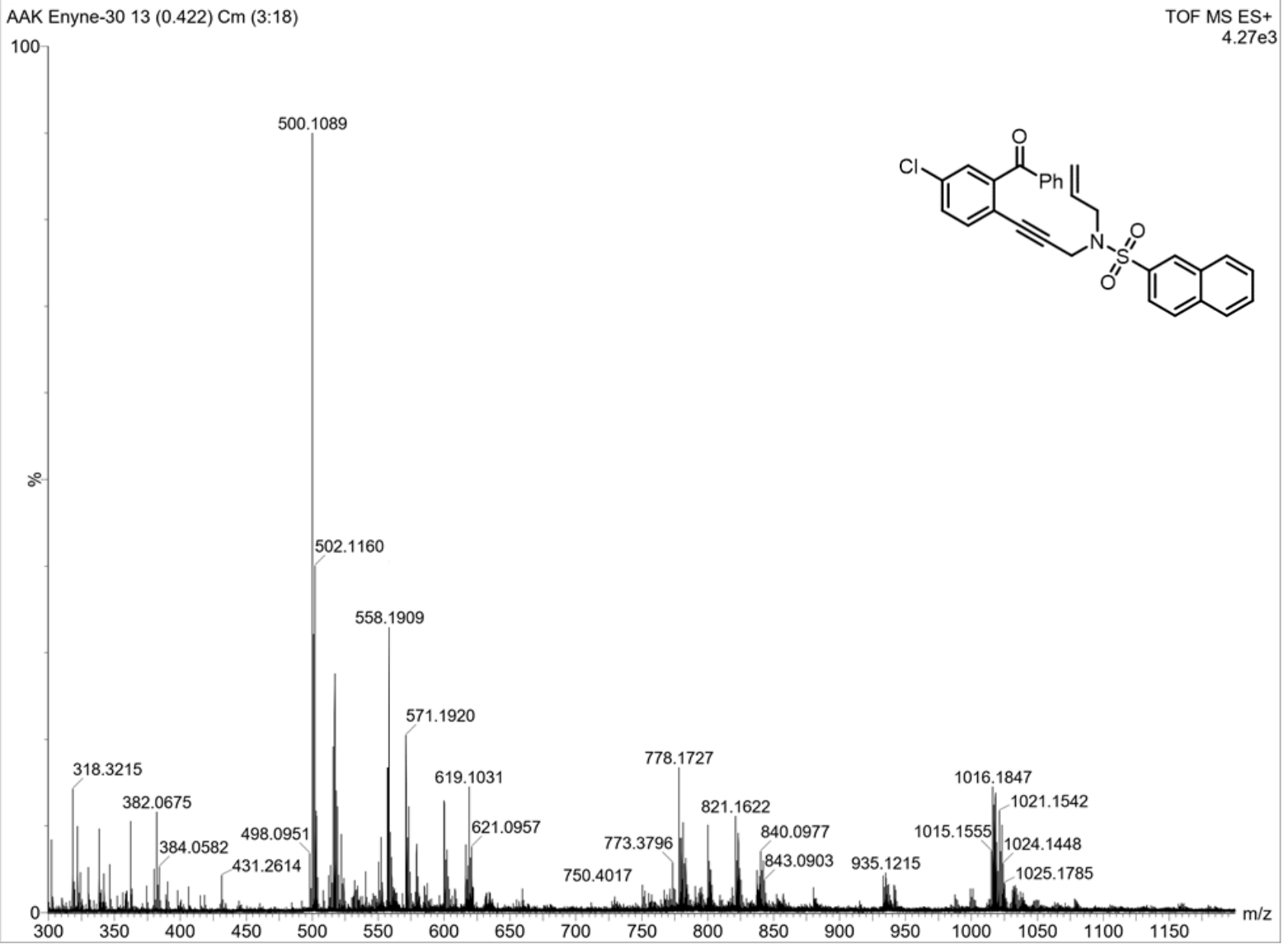

Mass-ESI Compound 3e 

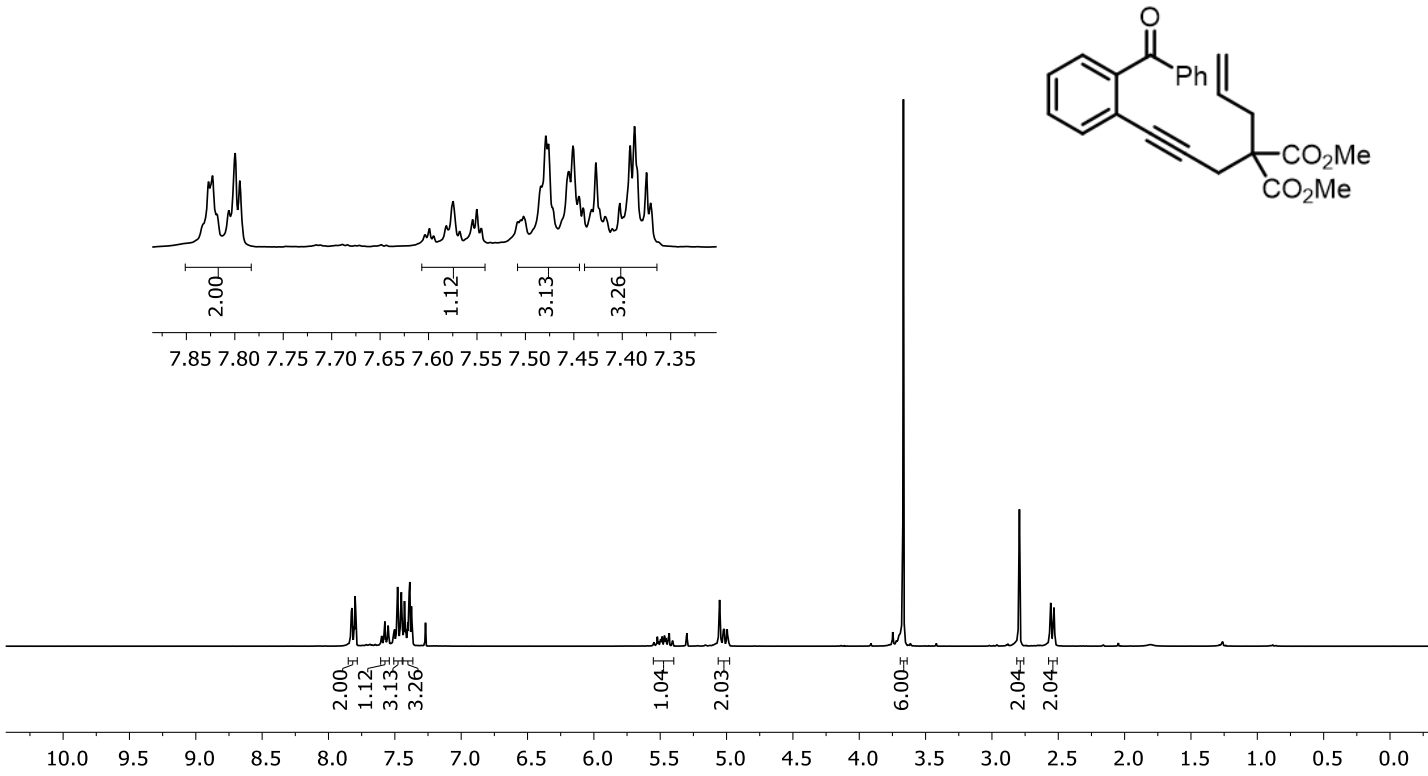

${ }^{1} \mathrm{H}$ NMR Compound $\mathbf{3 f}\left(300 \mathrm{MHz}, \mathrm{CDCl}_{3}\right)$

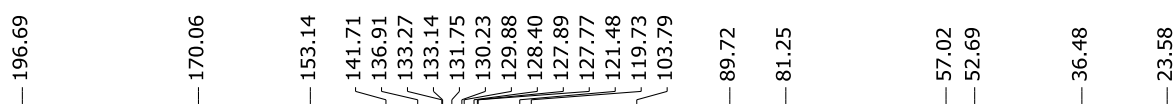
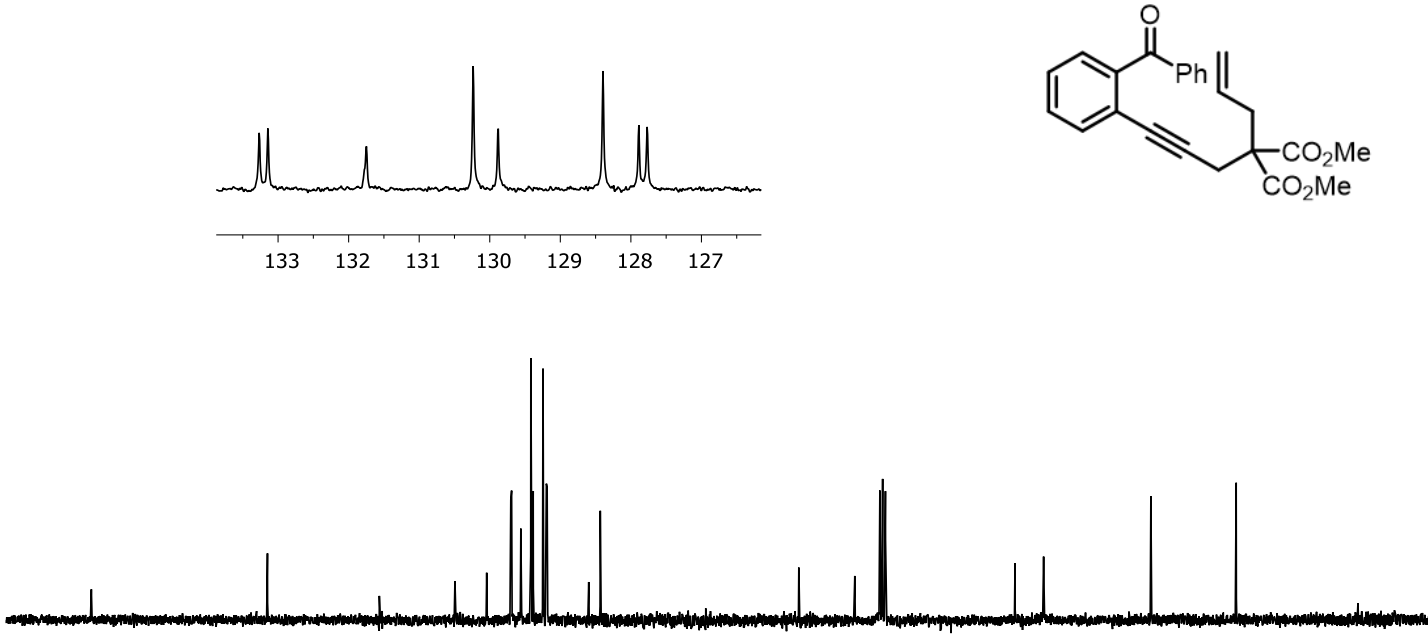

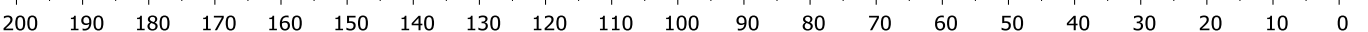

${ }^{13} \mathrm{C}$ NMR Compound $\mathbf{3 f}\left(75 \mathrm{MHz}, \mathrm{CDCl}_{3}\right)$ 


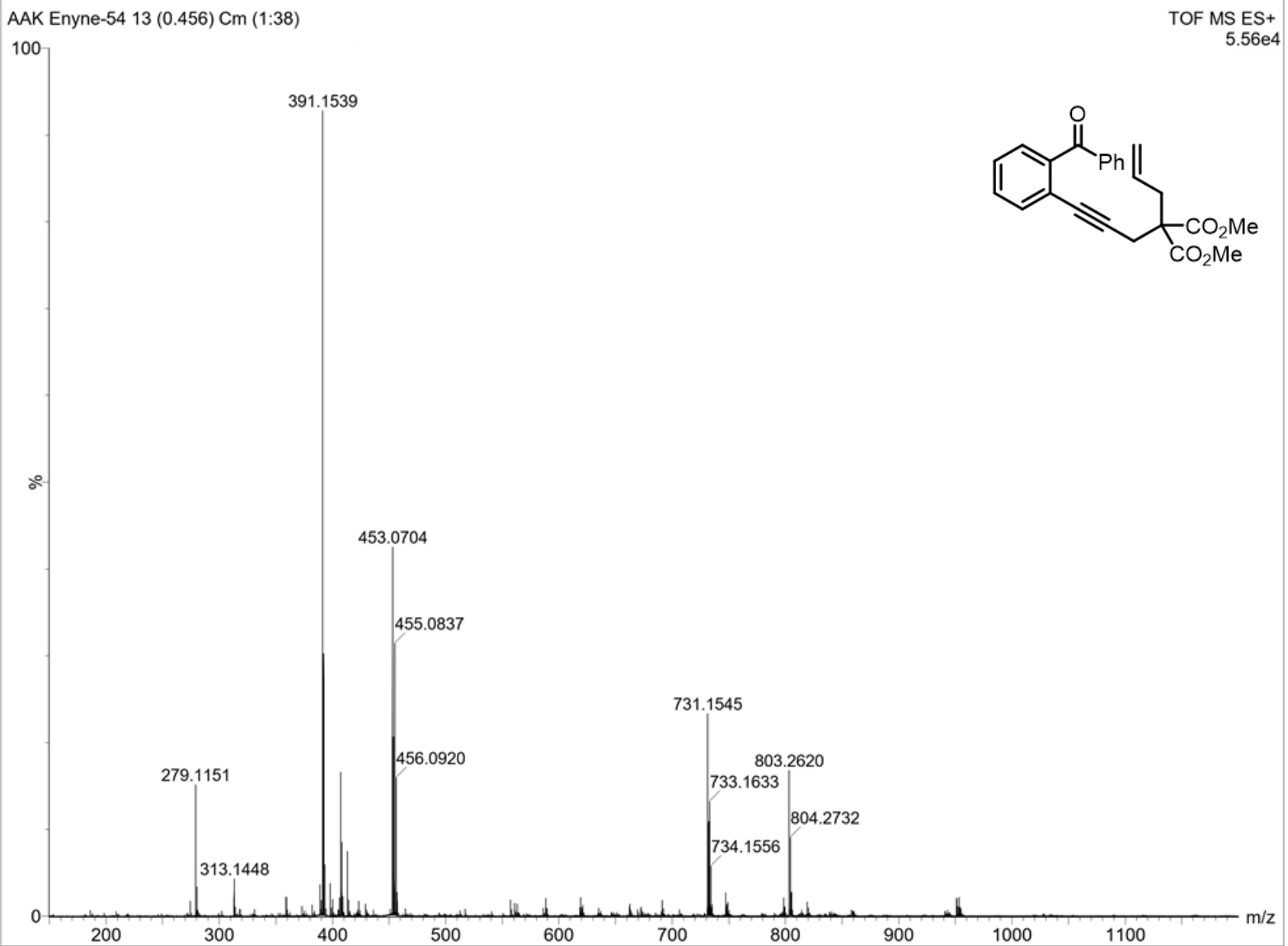

Mass-ESI Compound 3f 

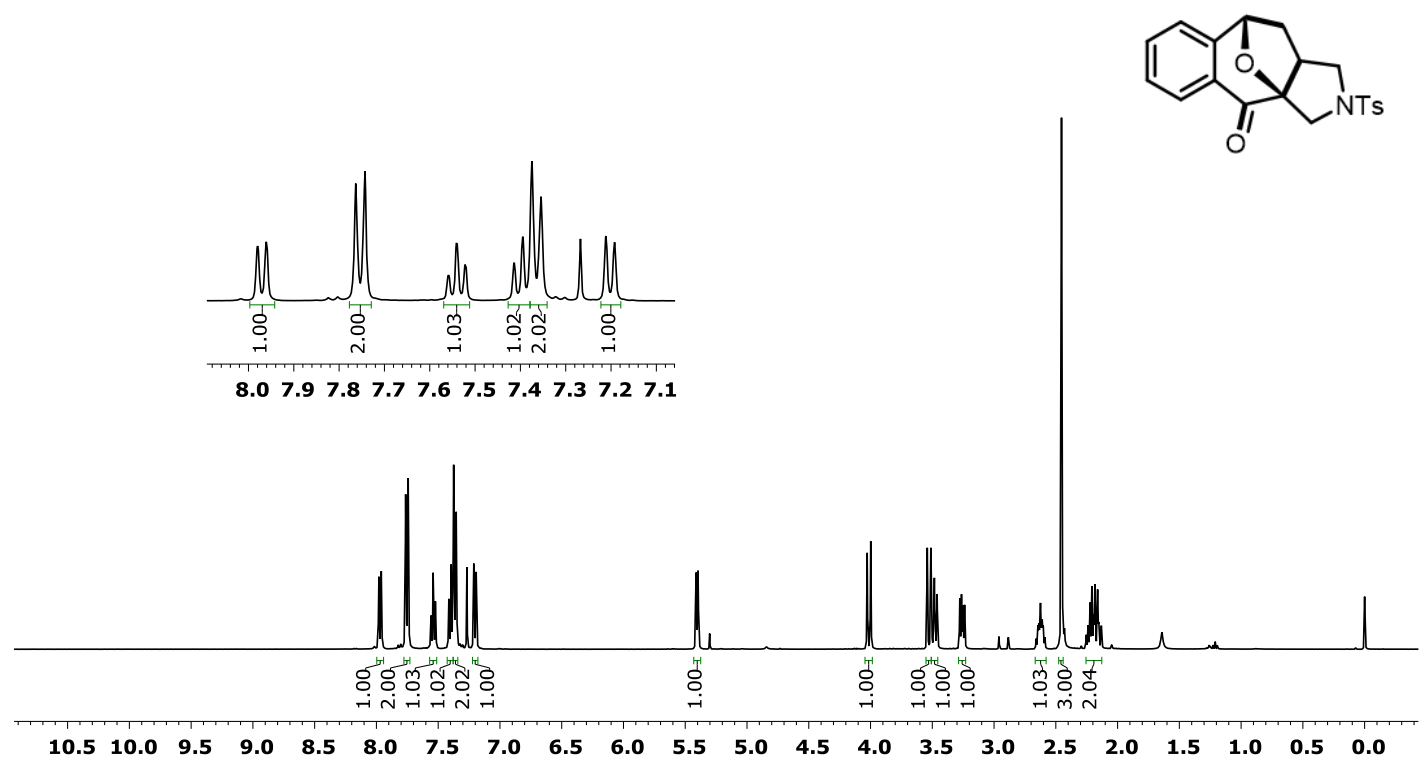

${ }^{1} \mathrm{H}$ NMR Compound 2a $\left(400 \mathrm{MHz}, \mathrm{CDCl}_{3}\right)$

$\underset{\substack{1 \\ \stackrel{1}{2}}}{\stackrel{1}{2}}$
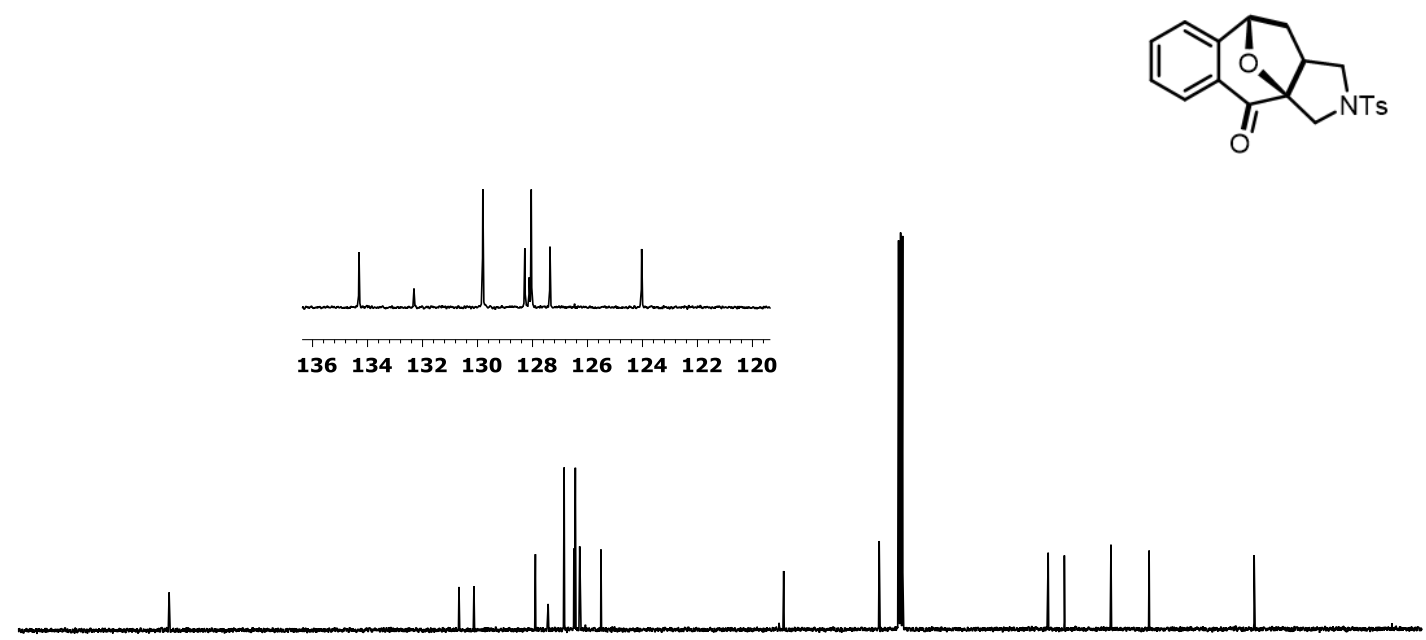

$\begin{array}{lllllllllllllllll}210 & 200 & 190 & 180 & 170 & 160 & 150 & 140 & 130 & 120 & 110 & 100 & 90 & 80 & 70 & 60 & 50\end{array}$

$\begin{array}{lllll}40 & 30 & 20 & 10 & 0\end{array}$

${ }^{13} \mathrm{C}$ NMR Compound $2 \mathbf{a}\left(100 \mathrm{MHz}, \mathrm{CDCl}_{3}\right)$ 


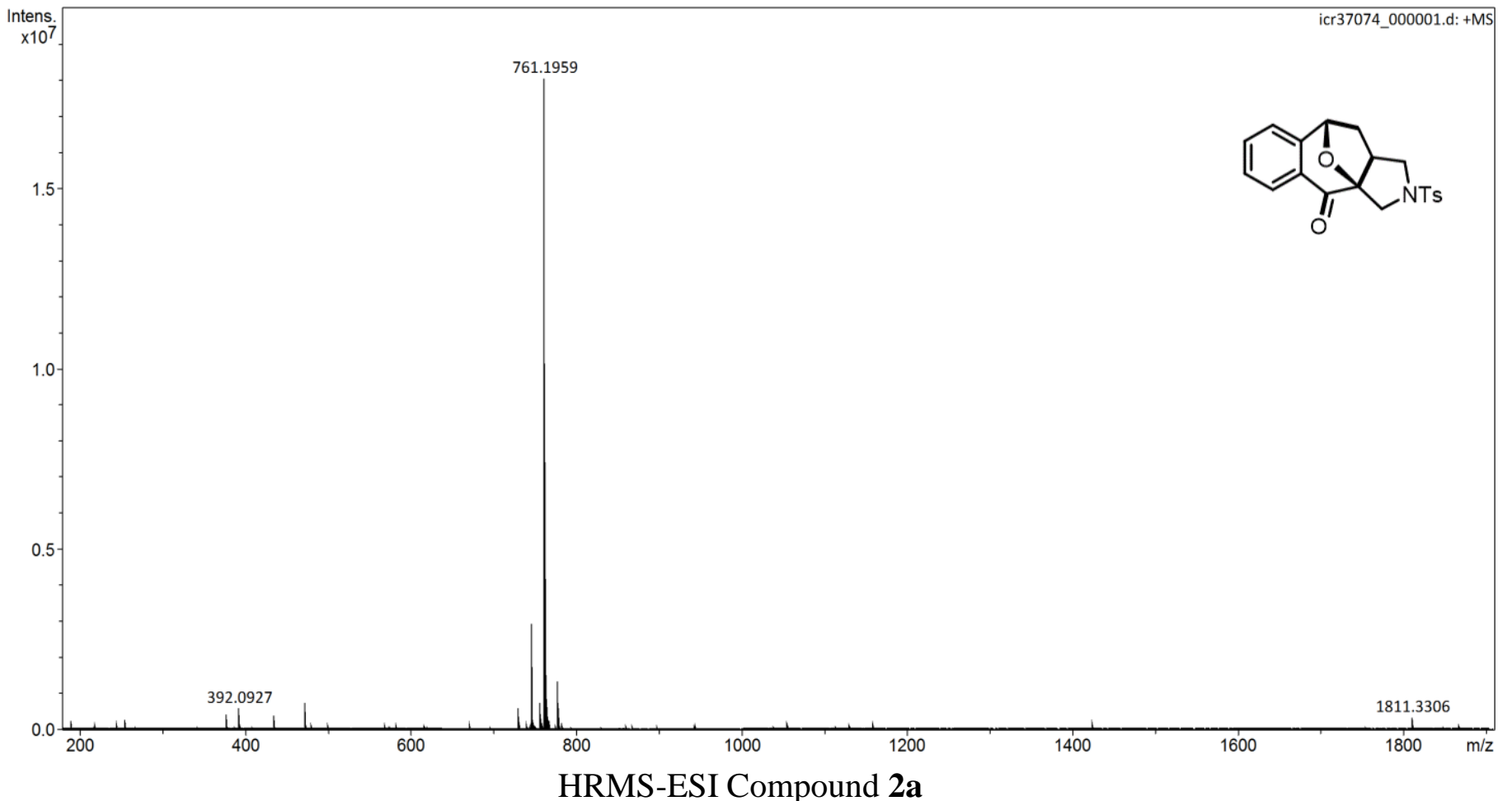

HRMS-ESI Compound 2a

\begin{tabular}{|c|c|c|c|c|c|c|c|}
\hline Meas. m/z & Ion Formula & $\mathrm{m} / \mathrm{z}$ & err [ppm] & mSigma & $\mathrm{rdb}$ & $\mathrm{e}^{-}$Conf & N-Rule \\
\hline \multirow[t]{5}{*}{392.0927} & $\mathrm{C} 20 \mathrm{H} 19 \mathrm{NNaO} 4 \mathrm{~S}$ & 392.0927 & 0.1 & 14.6 & 11.5 & even & ok \\
\hline & $\mathrm{C} 19 \mathrm{H} 20 \mathrm{O} 7 \mathrm{~S}$ & 392.0924 & -0.6 & 15.5 & 10.0 & odd & ok \\
\hline & $\mathrm{C} 26 \mathrm{H} 13 \mathrm{~N} 2 \mathrm{NaO}$ & 392.0920 & -1.7 & 36.3 & 21.0 & odd & ok \\
\hline & C26H10N5 & 392.0931 & 1.0 & 37.1 & 24.5 & even & ok \\
\hline & $\mathrm{C} 18 \mathrm{H} 14 \mathrm{~N} 7 \mathrm{O} 2 \mathrm{~S}$ & 392.0924 & -0.6 & 103.1 & 15.5 & even & ok \\
\hline \multirow[t]{3}{*}{761.1959} & $\mathrm{C} 40 \mathrm{H} 35 \mathrm{~N} 5 \mathrm{O} 7 \mathrm{~S} 2$ & 761.1972 & 1.8 & 21.7 & 26.0 & odd & ok \\
\hline & $\mathrm{C} 40 \mathrm{H} 30 \mathrm{~N} 6 \mathrm{NaO} 9$ & 761.1966 & 1.0 & 22.2 & 28.5 & even & ok \\
\hline & $\mathrm{C} 40 \mathrm{H} 38 \mathrm{~N} 2 \mathrm{NaO} 8 \mathrm{~S} 2$ & 761.1962 & 0.4 & 22.6 & 22.5 & even & ok \\
\hline
\end{tabular}



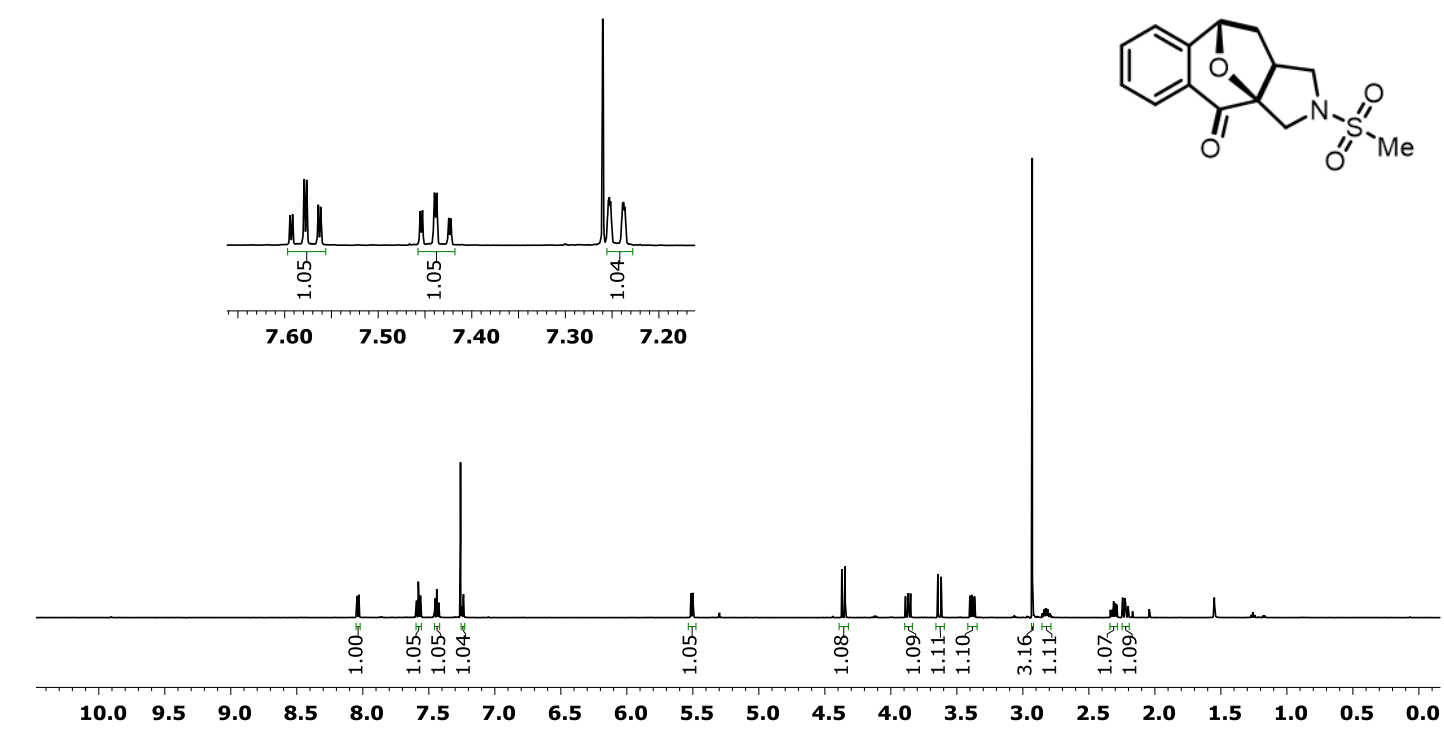

${ }^{1} \mathrm{H}$ NMR Compound $2 \mathbf{b}\left(500 \mathrm{MHz}, \mathrm{CDCl}_{3}\right)$

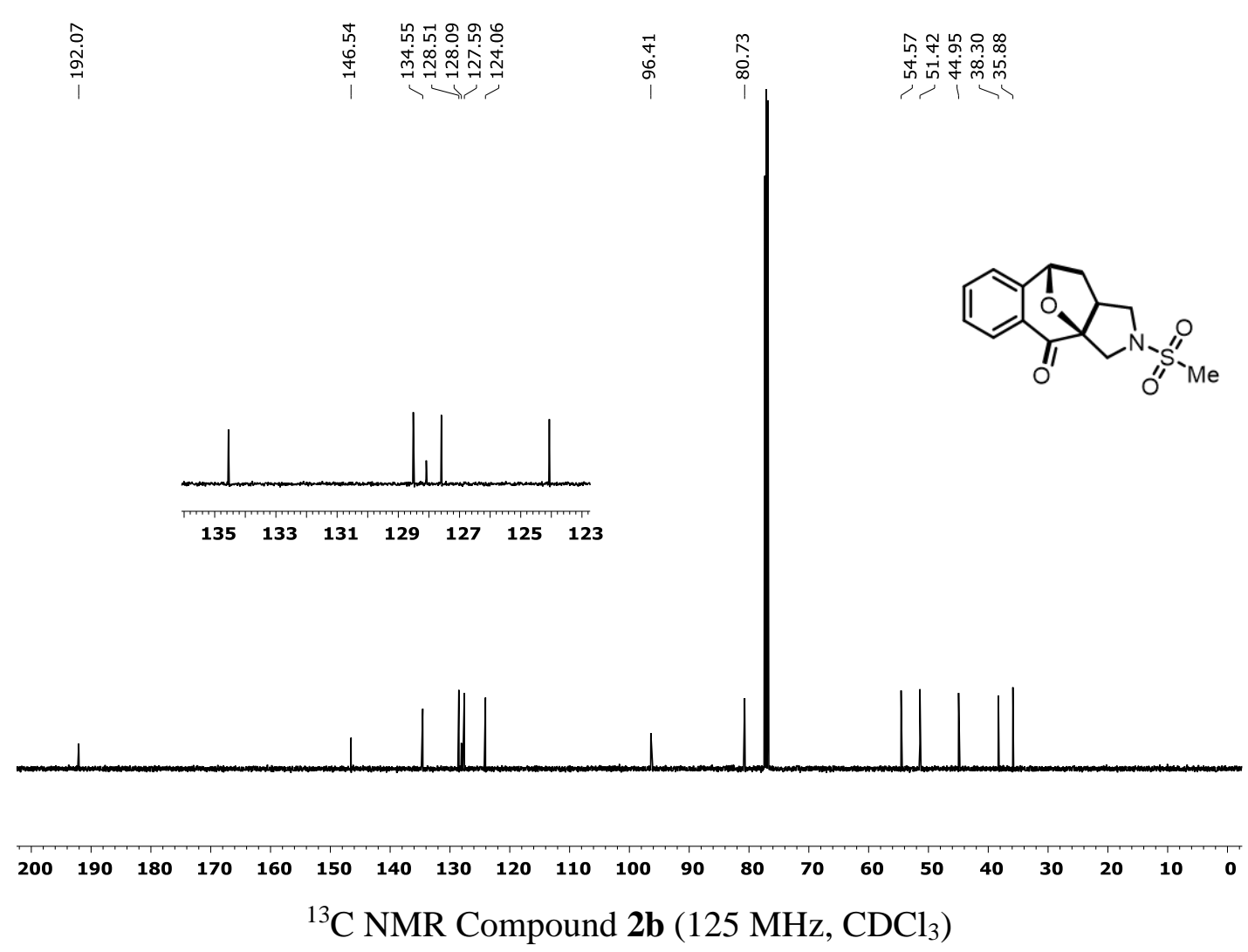




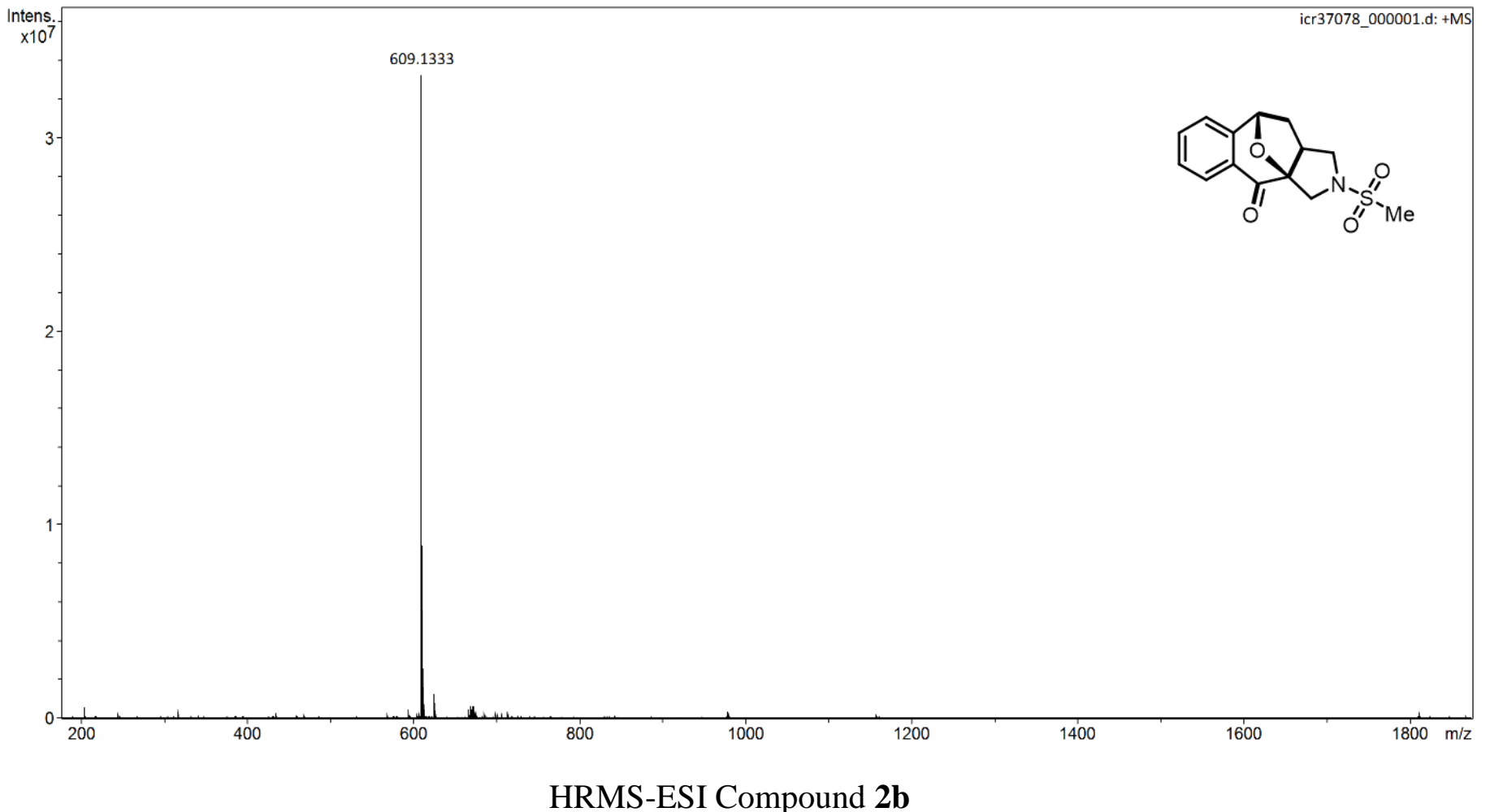

HRMS-ESI Compound 2b

$\begin{array}{ccrrrrrr}\text { Meas. } \mathrm{m} / \mathrm{z} & \text { Ion Formula } & \mathrm{m} / \mathrm{z} & \text { err [ppm] } & \text { mSigma } & \text { rdb } & \mathrm{e}^{-} \text {Conf } & \begin{array}{r}\text { N-Rule } \\ \text { 316.0613 }\end{array} \\ & \text { C20H9N2NaO } & 316.0607 & -1.9 & 13.1 & 17.0 & \text { odd } & \text { ok } \\ & \text { C20H6N5 } & 316.0618 & 1.5 & 15.4 & 20.5 & \text { even } & \text { ok } \\ & \text { C14H15NNaO4S } & 316.0614 & 0.3 & 26.8 & 7.5 & \text { even } & \text { ok } \\ 609.1333 & \text { C13H16O7S } & 316.0611 & -0.5 & 32.1 & 6.0 & \text { odd } & \text { ok } \\ & \text { C28H30N2NaO8S2 } & 609.1336 & 0.4 & 19.1 & 14.5 & \text { even } & \text { ok }\end{array}$



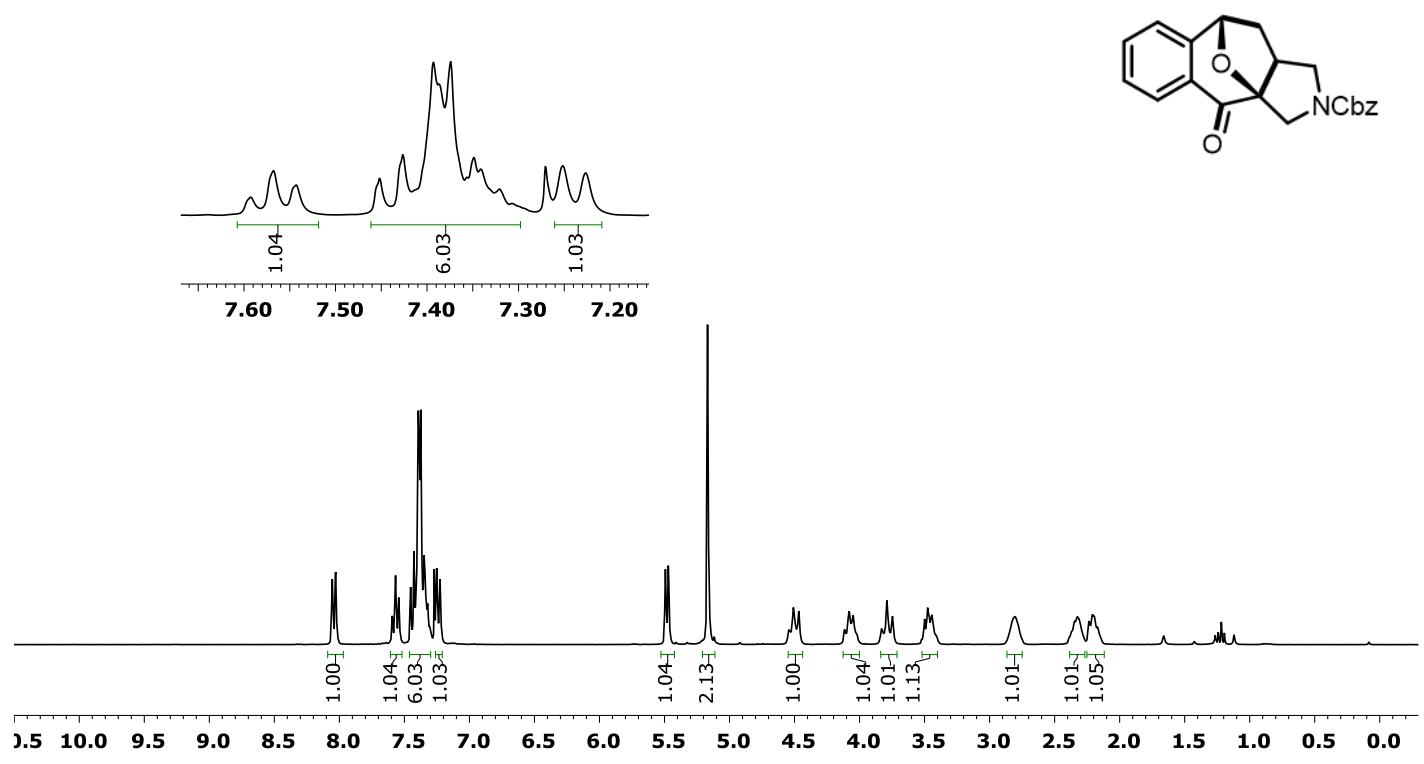

${ }^{1} \mathrm{H}$ NMR Compound 2c (300 $\left.\mathrm{MHz}, \mathrm{CDCl}_{3}\right)$

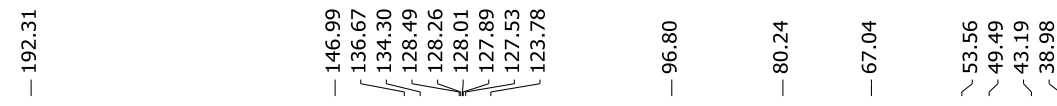
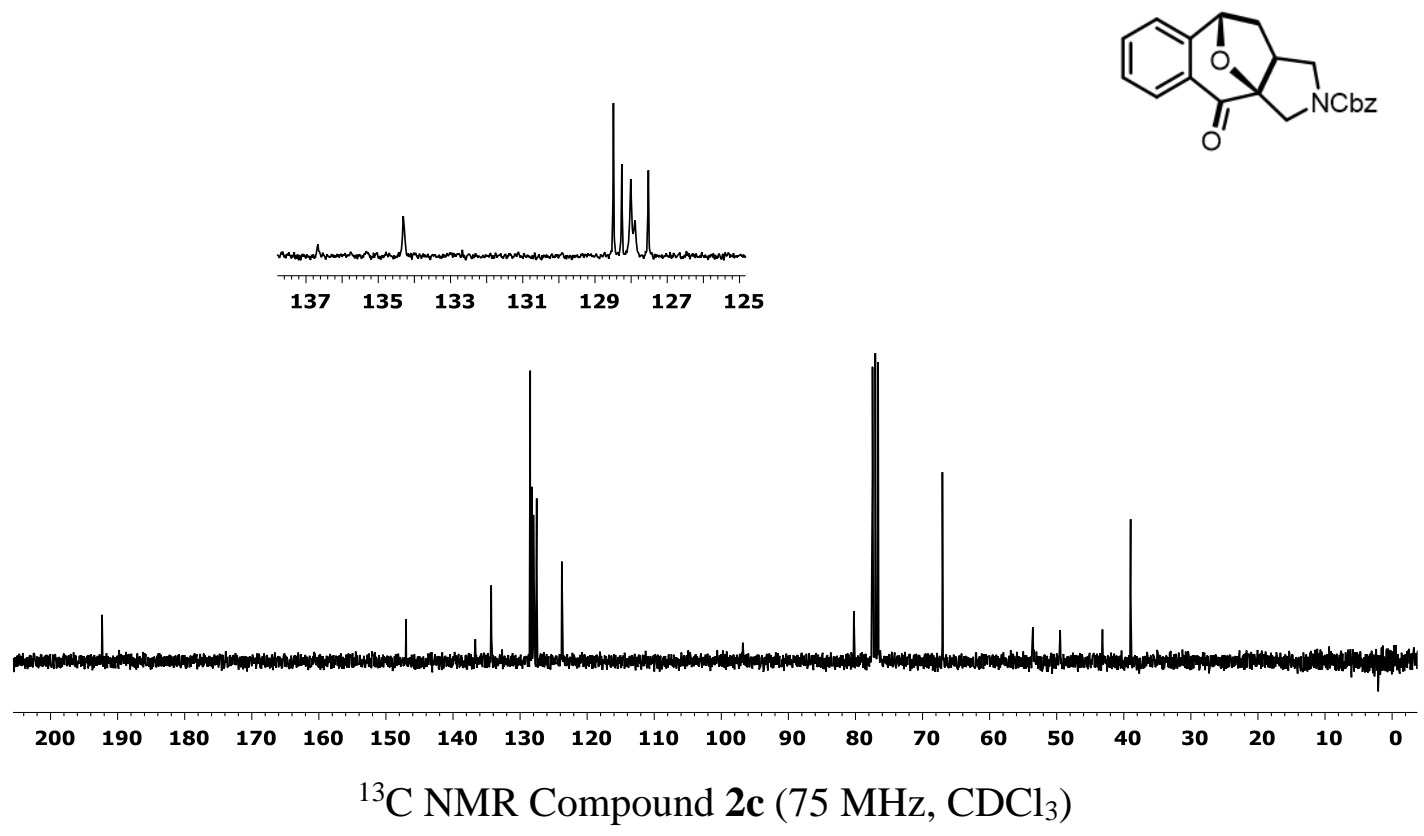
hobta27shr1 \#1 RT: 0.02 AV: 1 NL: 1.38E7

T: FTMS + p ESI Full ms [100.00-800.00]

306.1485

$\mathrm{C}_{20} \mathrm{H}_{20} \mathrm{O}_{2} \mathrm{~N}=306.1489$

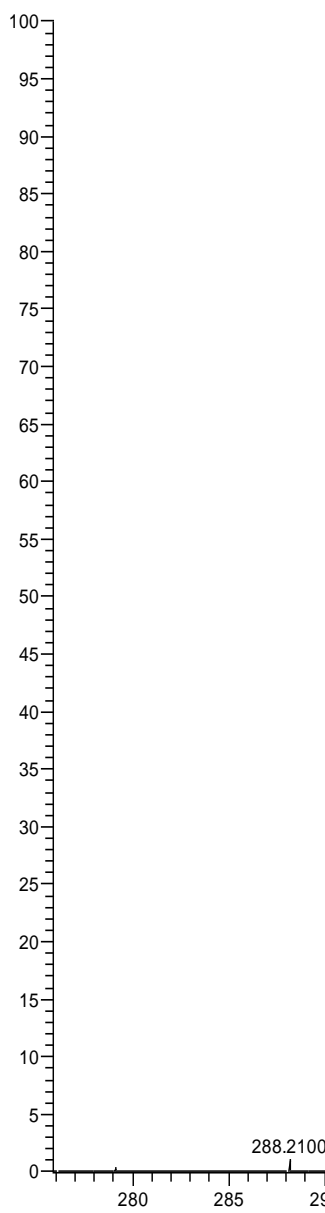

$-1.0660 \mathrm{ppm}$

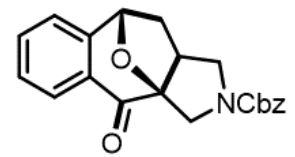

350.1382

$\mathrm{C}_{21} \mathrm{H}_{20} \mathrm{O}_{4} \mathrm{~N}=350.1387$

$-1.2566 \mathrm{ppm}$

367.1649 $\mathrm{C}_{21} \mathrm{H}_{23} \mathrm{O}_{4} \mathrm{~N}_{2}=367.1652$ $-0.7794 \mathrm{ppm}$ 


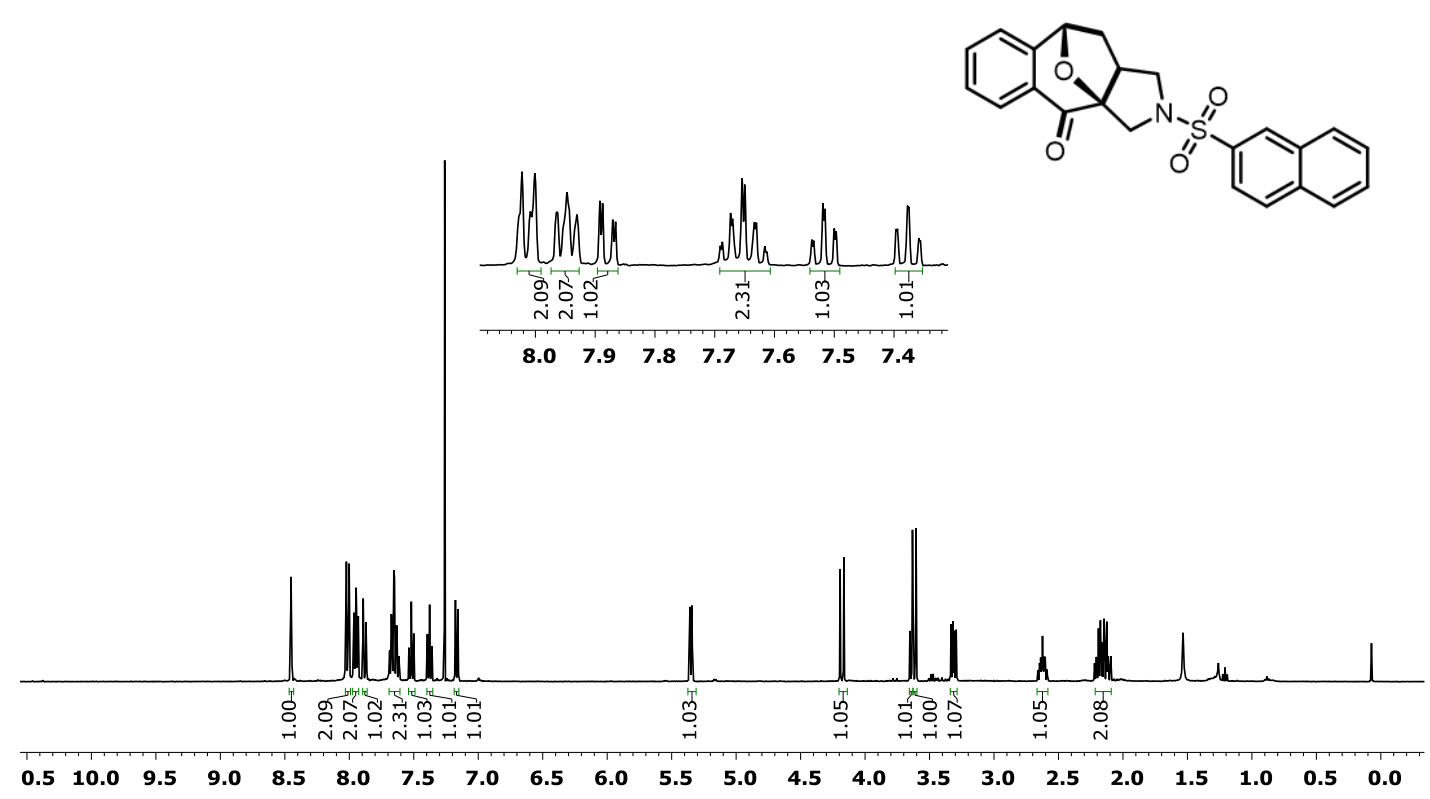

${ }^{1} \mathrm{H}$ NMR Compound $2 \mathbf{d}\left(400 \mathrm{MHz}, \mathrm{CDCl}_{3}\right)$

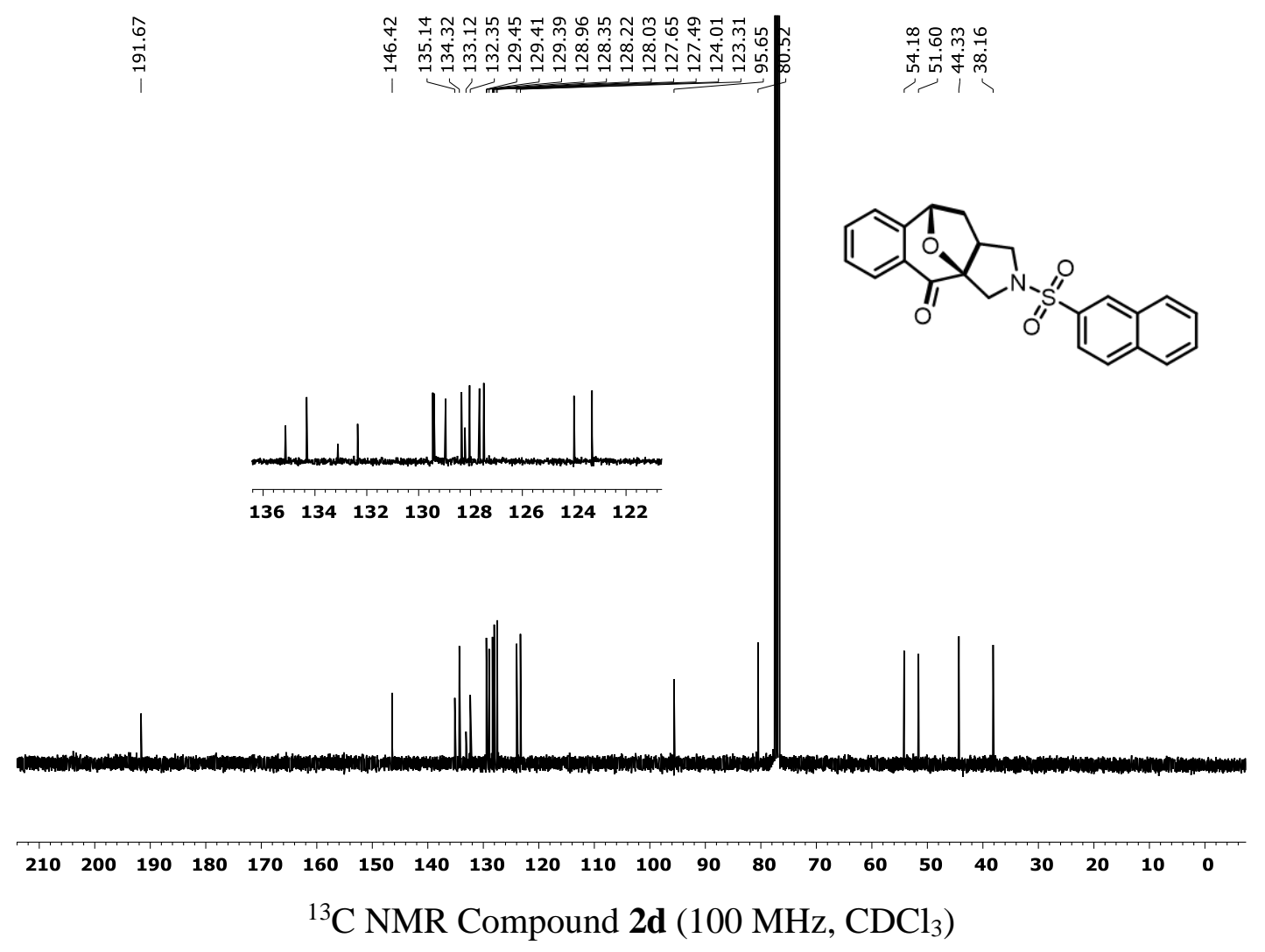




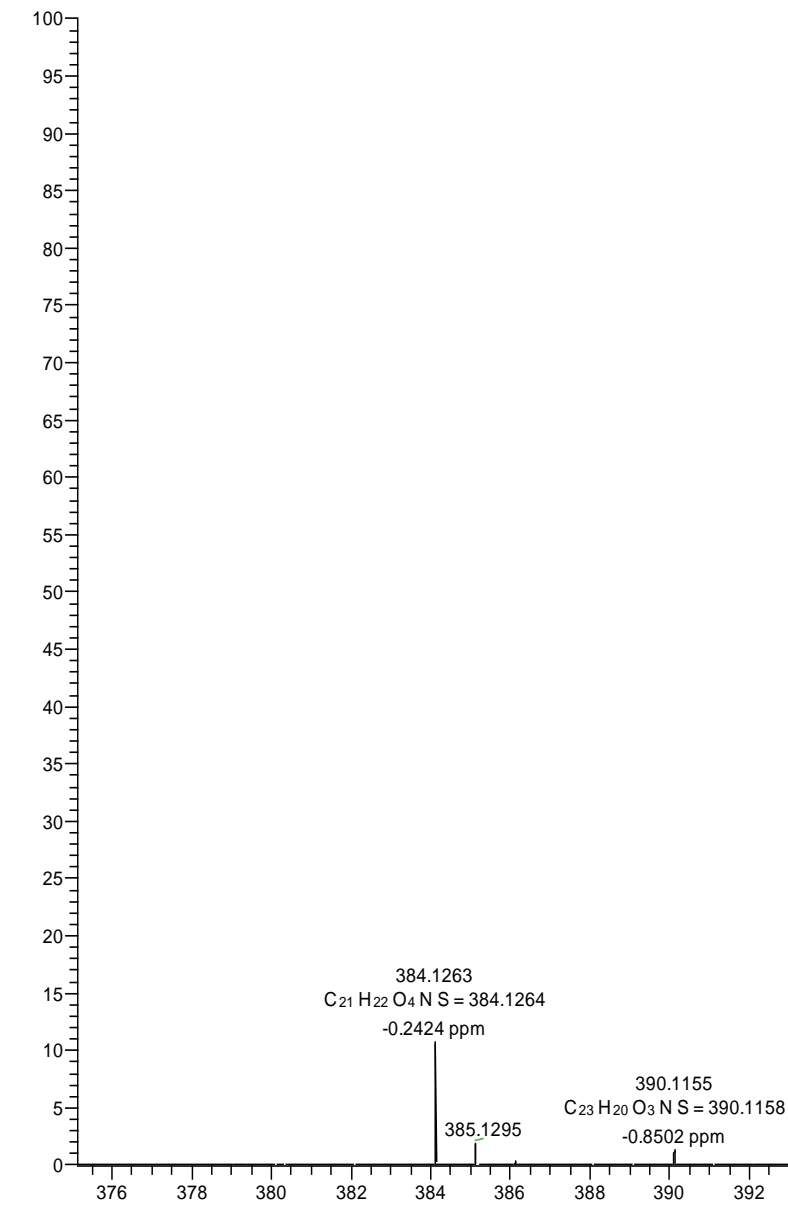
$0.9596 \mathrm{ppm}$

HRMS-ESI Compound 2d 

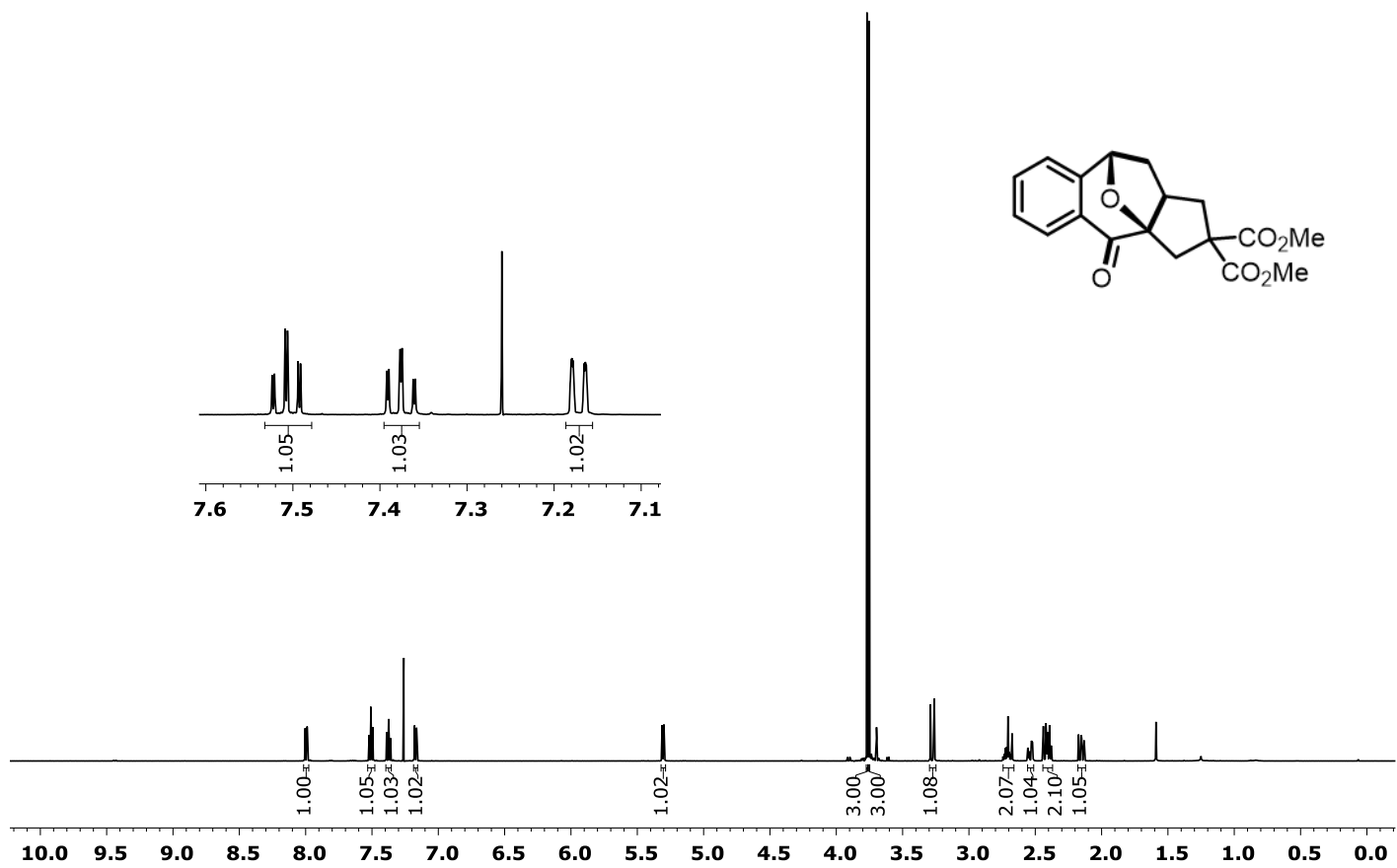

${ }^{1} \mathrm{H}$ NMR Compound $2 \mathrm{e}\left(500 \mathrm{MHz}, \mathrm{CDCl}_{3}\right)$

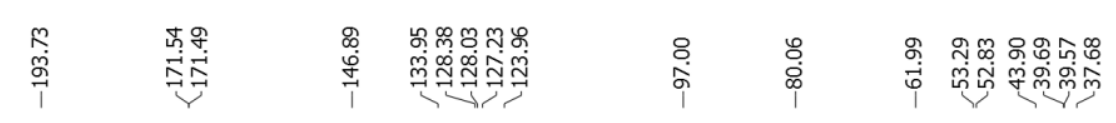

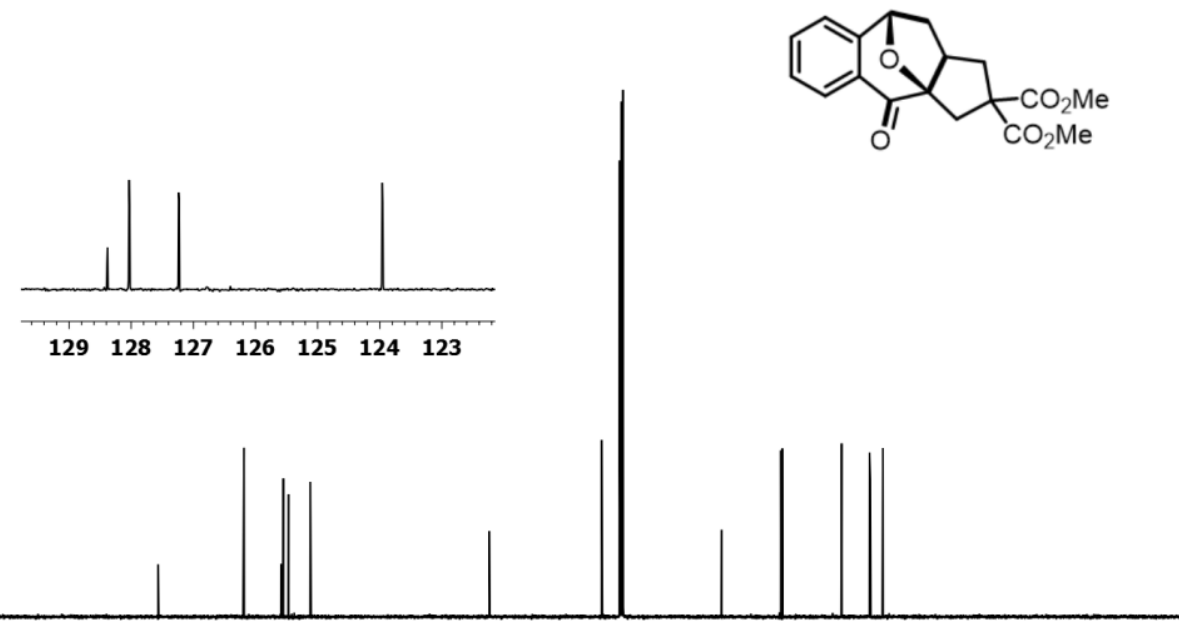

$\begin{array}{lllllllllllllllllllll}200 & 190 & 180 & 170 & 160 & 150 & 140 & 130 & 120 & 110 & 100 & 90 & 80 & 70 & 60 & 50 & 40 & 30 & 20 & 10 & 0\end{array}$

${ }^{13} \mathrm{C}$ NMR Compound $2 \mathbf{e}\left(125 \mathrm{MHz}, \mathrm{CDCl}_{3}\right)$ 


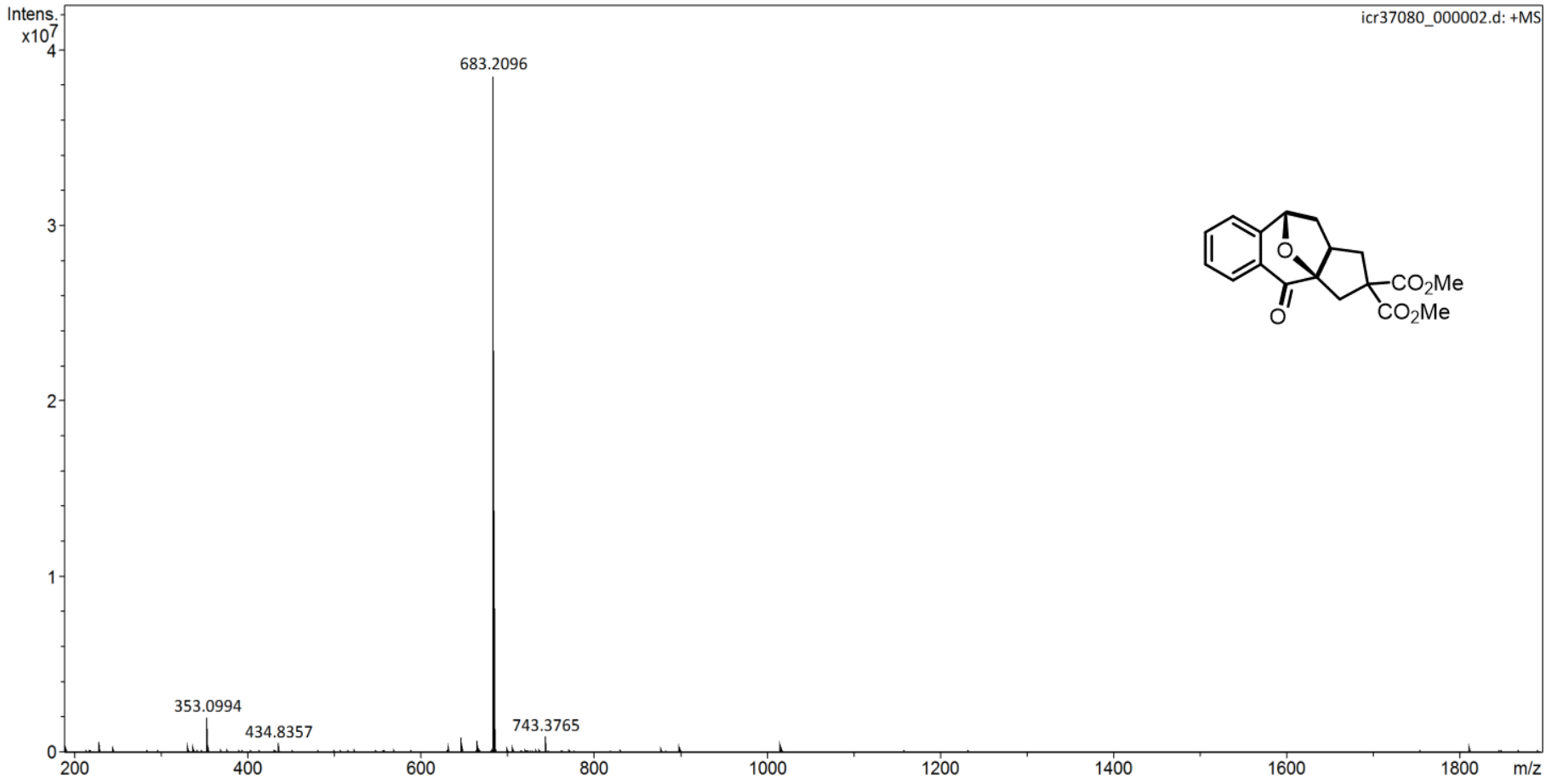

HRMS-ESI Compound 2e

$\begin{array}{ccrrrrrr}\text { Meas. } \mathrm{m} / \mathrm{z} & \text { Ion Formula } & \mathrm{m} / \mathrm{z} & \text { err }[\mathrm{ppm}] & \mathrm{mSigma} & \mathrm{rdb} & \mathrm{e}^{-} \text {Conf } & \begin{array}{r}\text { N-Rule } \\ \text { 331.1176 }\end{array} \\ & \text { C18H19O6 } & 331.1176 & -0.1 & 11.6 & 9.5 & \text { even } & \text { ok } \\ 353.0994 & \text { C17H13N7O } & 331.1176 & -0.1 & 18.0 & 15.0 & \text { odd } & \text { ok } \\ & \text { C18H18NaO6 } & 353.0996 & 0.5 & 12.3 & 9.5 & \text { even } & \text { ok } \\ & \text { C16H13N6O4 } & 353.0993 & -0.3 & 13.3 & 13.5 & \text { even } & \text { ok } \\ & \text { C17H12N7NaO } & 353.0996 & 0.5 & 15.1 & 15.0 & \text { odd } & \text { ok } \\ 683.2096 & \text { C36H33N3O11 } & 683.2110 & 2.0 & 27.3 & 22.0 & \text { odd } & \text { ok } \\ & \text { C36H36NaO12 } & 683.2099 & 0.5 & 31.2 & 18.5 & \text { even } & \text { ok } \\ & \text { C49H25N5 } & 683.2104 & 1.3 & 87.5 & 40.0 & \text { odd } & \text { ok } \\ & \text { C48H29NO4 } & 683.2091 & -0.7 & 94.5 & 35.0 & \text { odd } & \text { ok } \\ & \text { C49H28N2NaO } & 683.2094 & -0.3 & 98.3 & 36.5 & \text { even } & \text { ok }\end{array}$




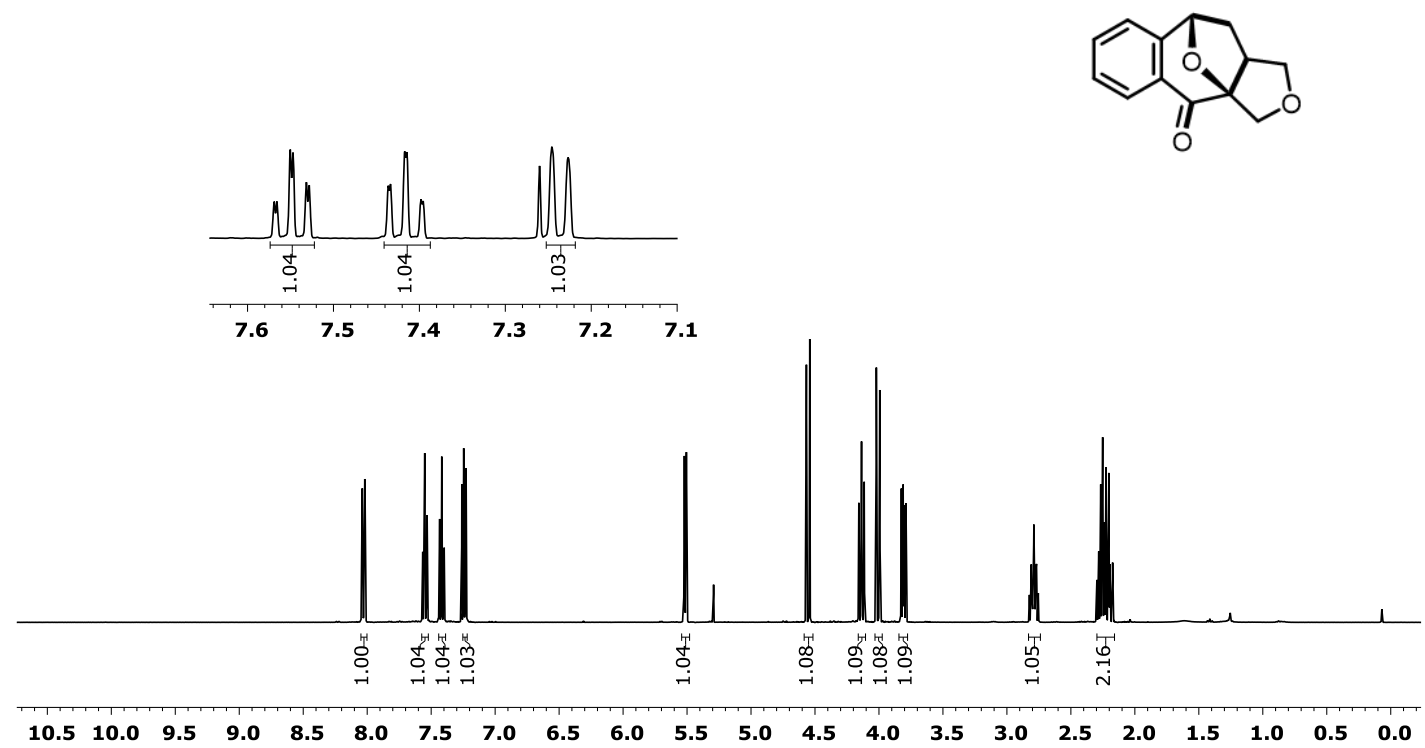

${ }^{1} \mathrm{H}$ NMR Compound $2 f\left(400 \mathrm{MHz}, \mathrm{CDCl}_{3}\right)$

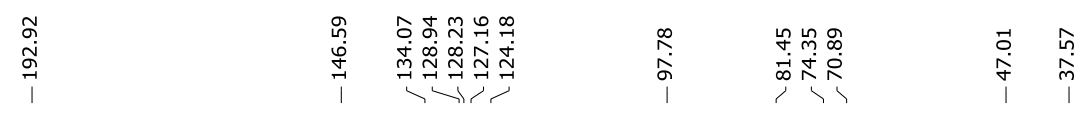

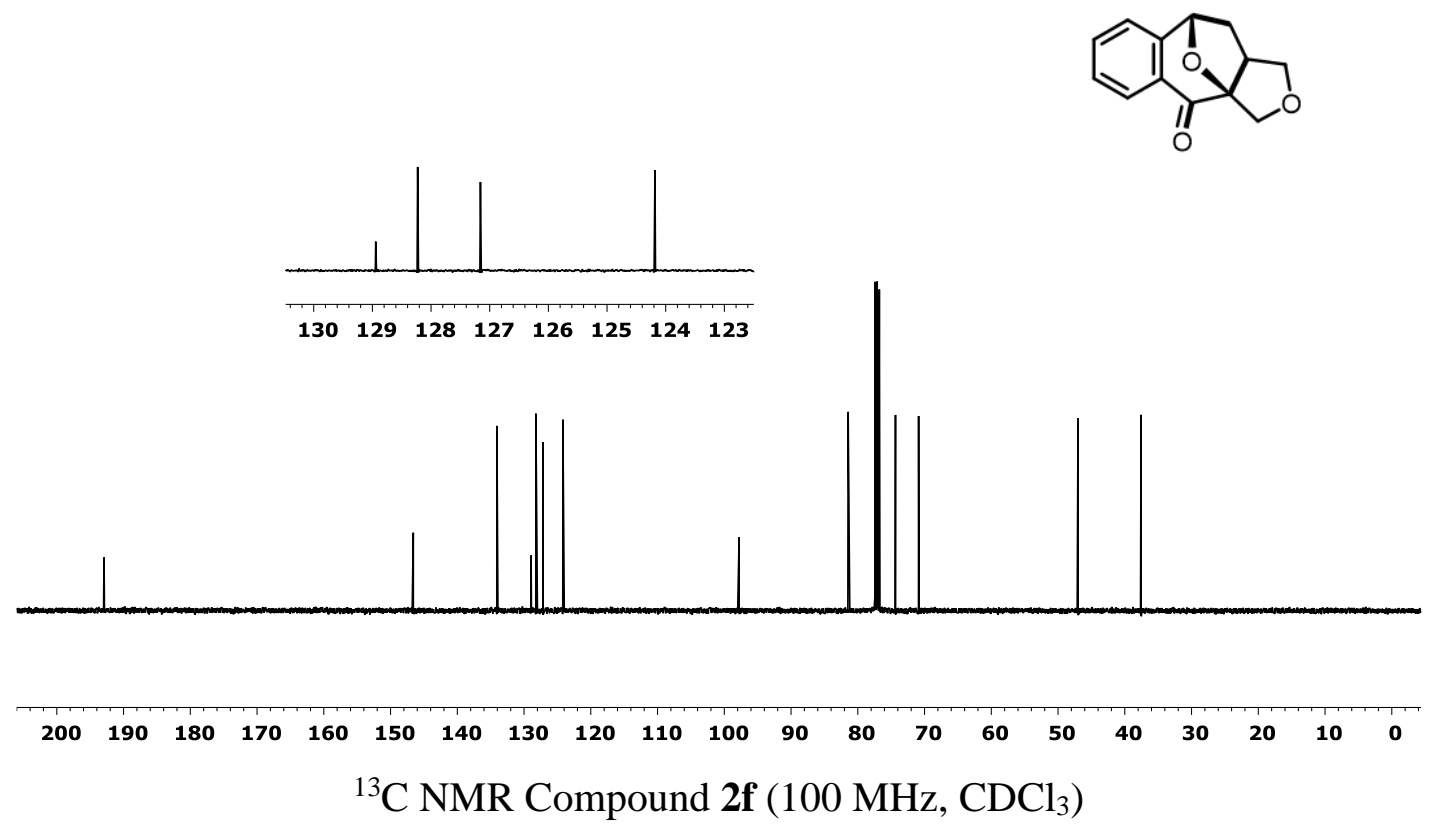




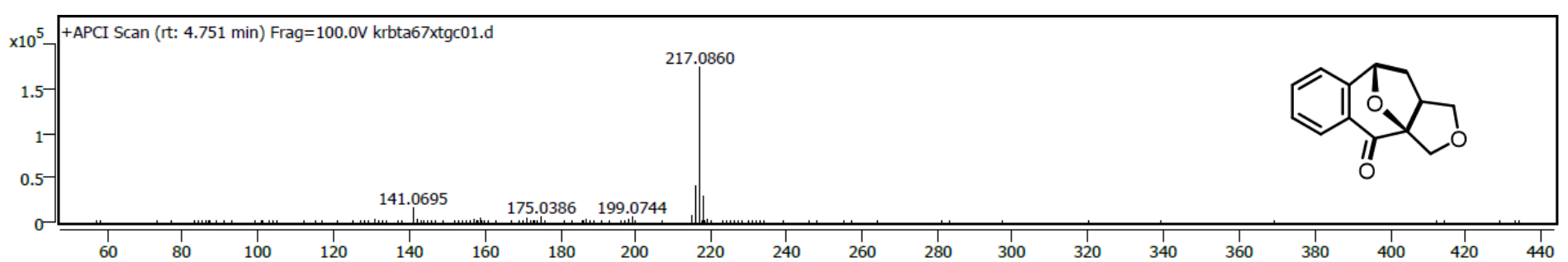

HRMS-ESI Compound $2 \mathbf{f}$

Spectrum Peaks

$\begin{array}{rrrrr}\mathbf{m} / \mathbf{z} & \mathbf{Z} & \text { Abund } & \text { Abund \% } & \mathbf{m} / \mathbf{z}(\mathbf{C a l c}) \\ 217.0860 & 1 & 174515 & 100.00 & 217.0859 \\ 218.0890 & 1 & 27921 & 16.00 & 218.0893 \\ 219.0903 & 1 & 2815 & 1.61 & 219.0917 \\ 128.0609 & 1051 & 0.60 & \\ 129.0704 & 1113 & 0.64 & \\ 131.0484 & 2460 & 1.41 & \\ 133.0279 & 1284 & 0.74 & \\ 141.0695 & 1 & 15871 & 9.09 & \\ 142.0731 & 1 & 2734 & 1.57 & \\ 145.0635 & 1115 & 0.64 & \\ 157.0644 & 2943 & 1.69 & \\ 158.0712 & 1301 & 0.75 & \\ 159.0435 & 3331 & 1.91 & \\ 159.0802 & 1676 & 0.96 & \\ 169.0637 & 1206 & 0.69 & \\ 171.0801 & 3942 & 2.26 & \\ 175.0386 & 4561 & 2.61 & \\ 187.0751 & 2814 & 1.61 & \\ 198.0664 & 2459 & 1.41 & \\ 199.0744 & 5294 & 3.03 & \\ 215.0695 & 6537 & 3.75 & \\ 216.0779 & 40354 & 23.12 & \\ 223.0637 & 1442 & 0.83 & \\ 231.0855 & 1006 & 0.58 & \end{array}$
Diff (ppm) Ion Species Formula
Ion Type

$\begin{array}{rrr}0.18 & (\mathrm{M}+\mathrm{H})+ & \mathrm{C} 13 \mathrm{H} 12 \mathrm{O} 3 \\ -1.42 & (\mathrm{M}+\mathrm{H})+ & \mathrm{C} 13 \mathrm{H} 12 \mathrm{O} 3\end{array}$

218.0890

$(\mathrm{M}+\mathrm{H})+$

$\mathrm{C} 13 \mathrm{H} 12 \mathrm{O} 3$ 

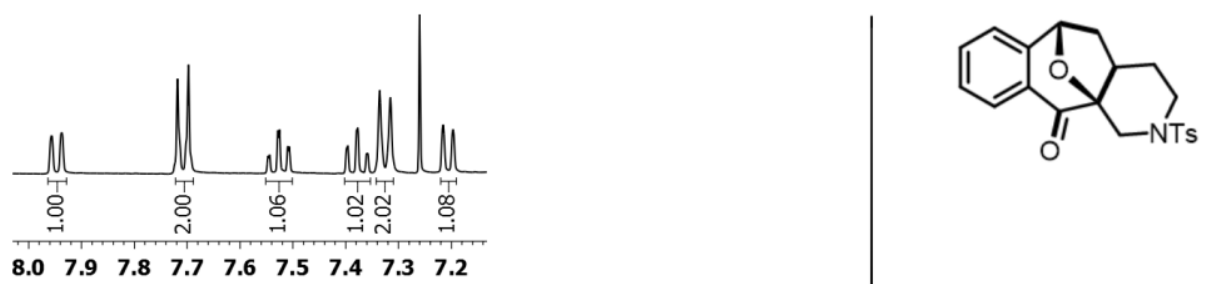

$\begin{array}{lllllllll}8.0 & 7.9 & 7.8 & 7.7 & 7.6 & 7.5 & 7.4 & 7.3 & 7.2\end{array}$

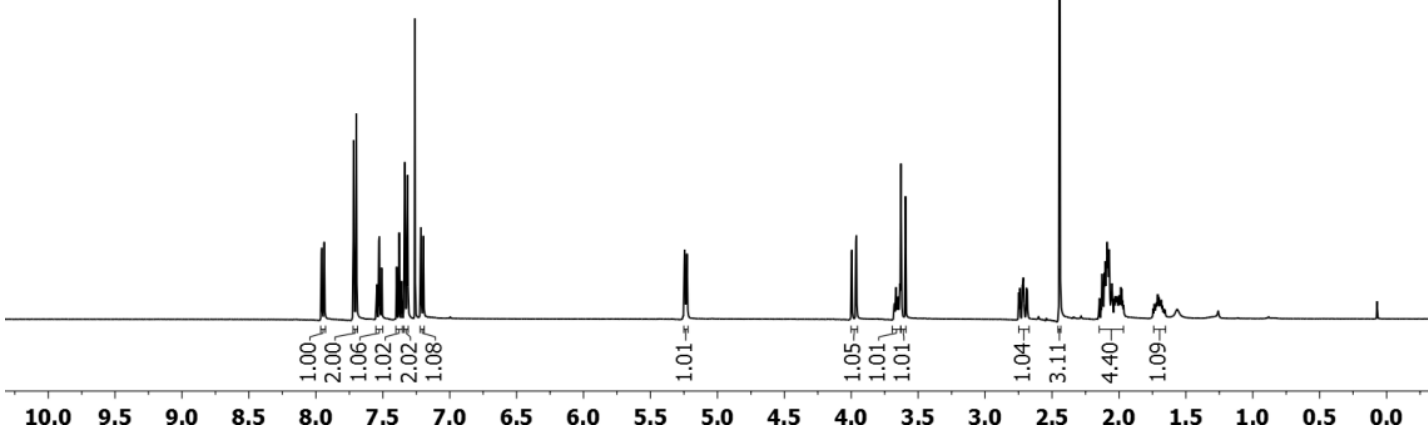

${ }^{1} \mathrm{H}$ NMR Compound 2g (400 MHz, $\mathrm{CDCl}_{3}$ )
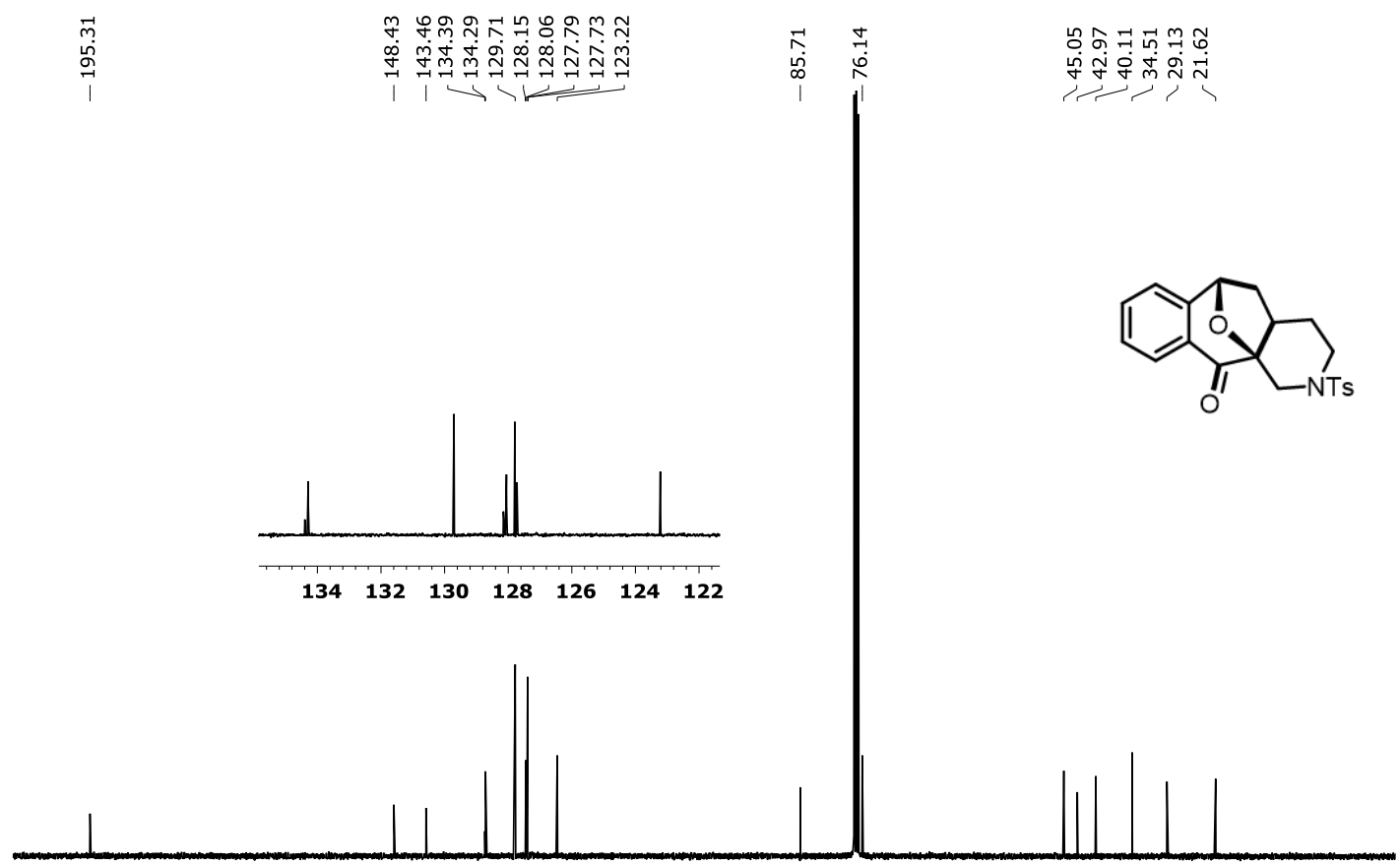

${ }^{13} \mathrm{C}$ NMR Compound $2 \mathrm{~g}\left(100 \mathrm{MHz}, \mathrm{CDCl}_{3}\right)$ 
hobta29shr1 \#1 RT: 0.02 AV: 1 NL: 1.96E7

T: FTMS + p ESI Full ms [100.00-700.00]

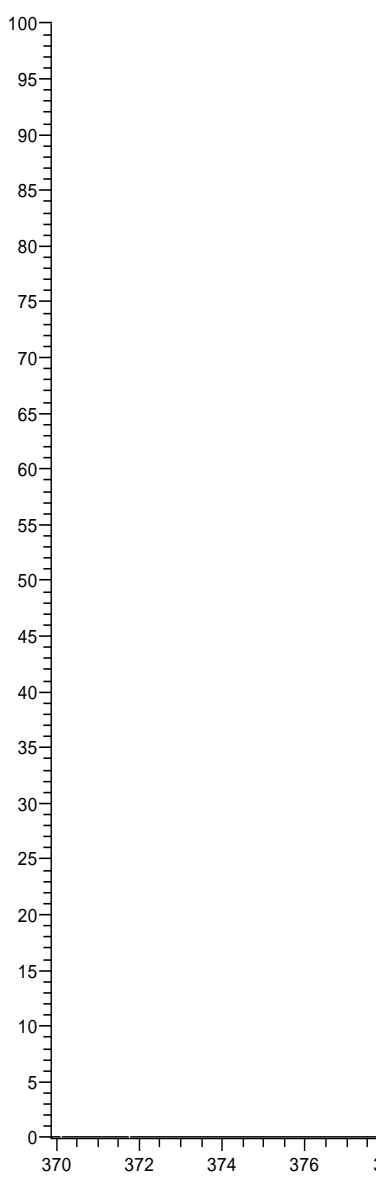

384.1264

$\mathrm{C}_{21} \mathrm{H}_{22} \mathrm{O}_{4} \mathrm{~N} \mathrm{~S}=384.1264$

$-0.0040 \mathrm{ppm}$

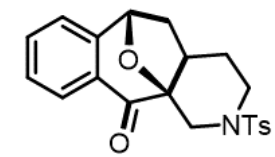

385.1297

$386.1220 \quad 0.8360 \mathrm{ppm}$

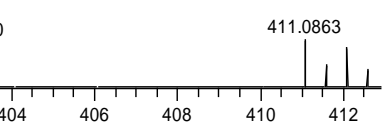

HRMS-ESI Compound 2g 


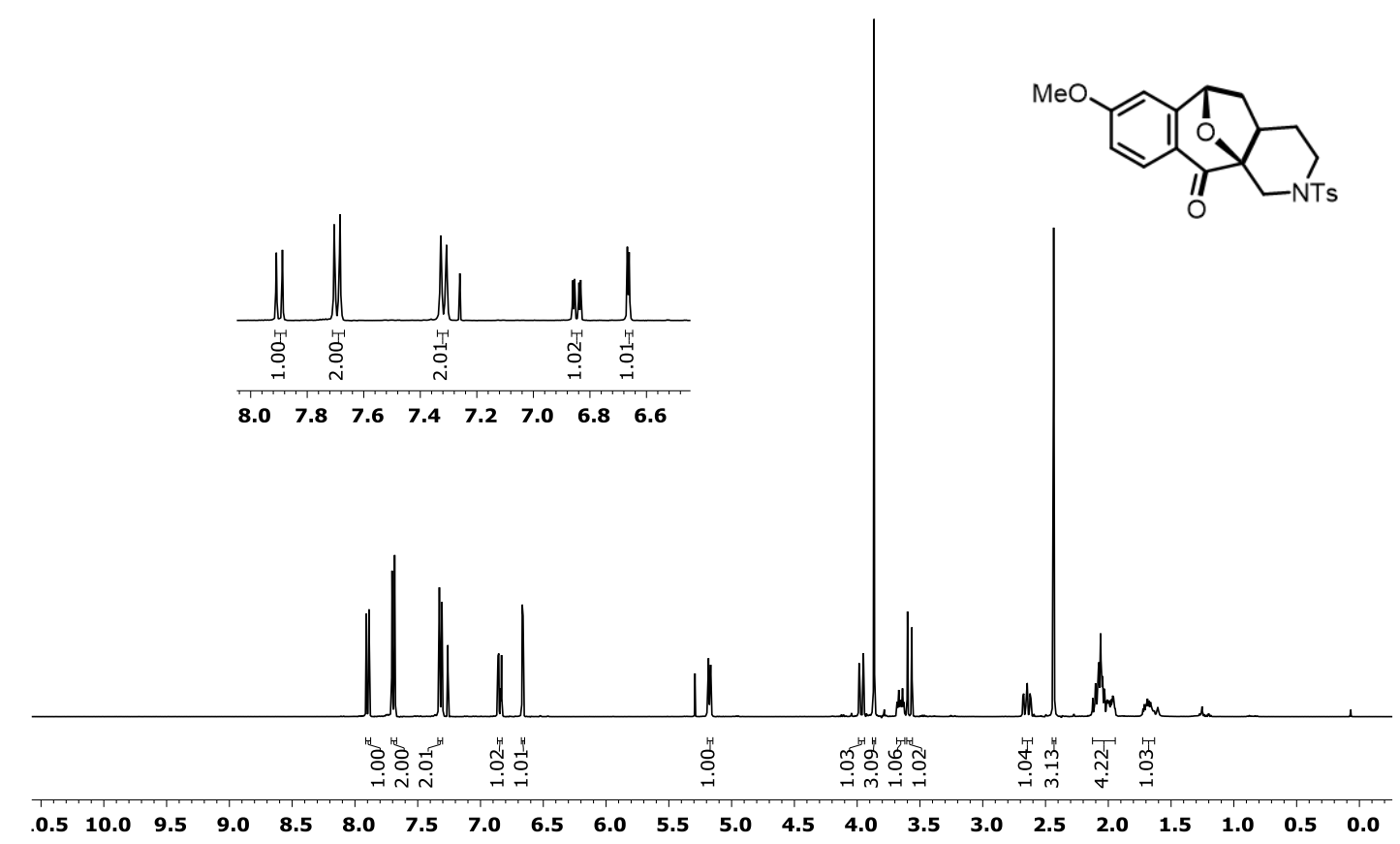

${ }^{1} \mathrm{H}$ NMR Compound $2 \mathbf{h}\left(400 \mathrm{MHz}, \mathrm{CDCl}_{3}\right)$

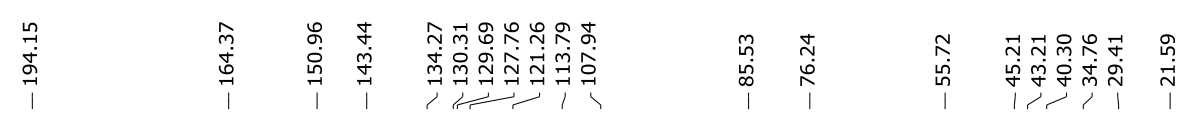
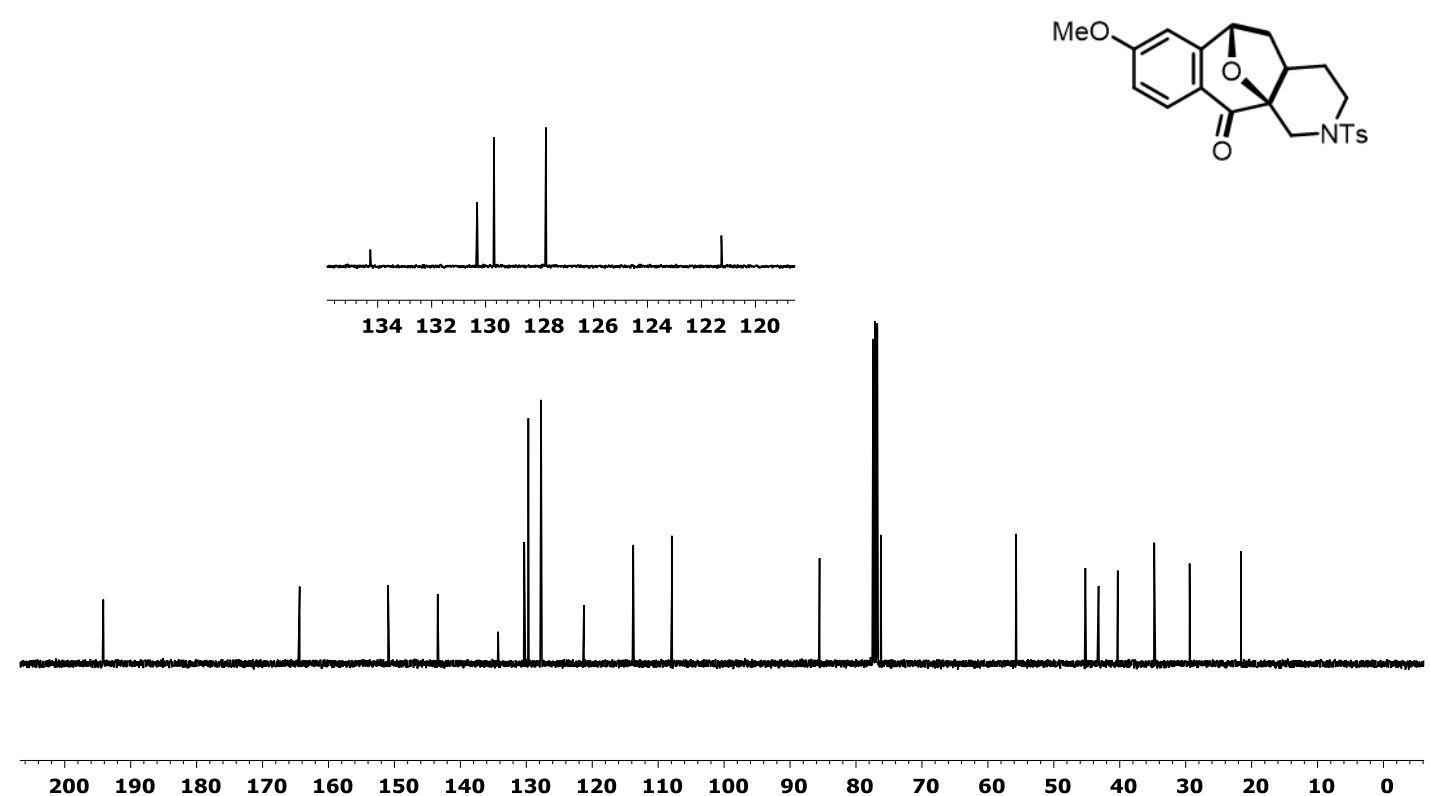

${ }^{13} \mathrm{C}$ NMR Compound $\mathbf{2 h}\left(100 \mathrm{MHz}, \mathrm{CDCl}_{3}\right)$ 
krbta58shr3 \#1 RT: 0.02 AV: 1 NL: 3.92E6 T: FTMS + p ESI Full lock ms [100.00-1000.00]

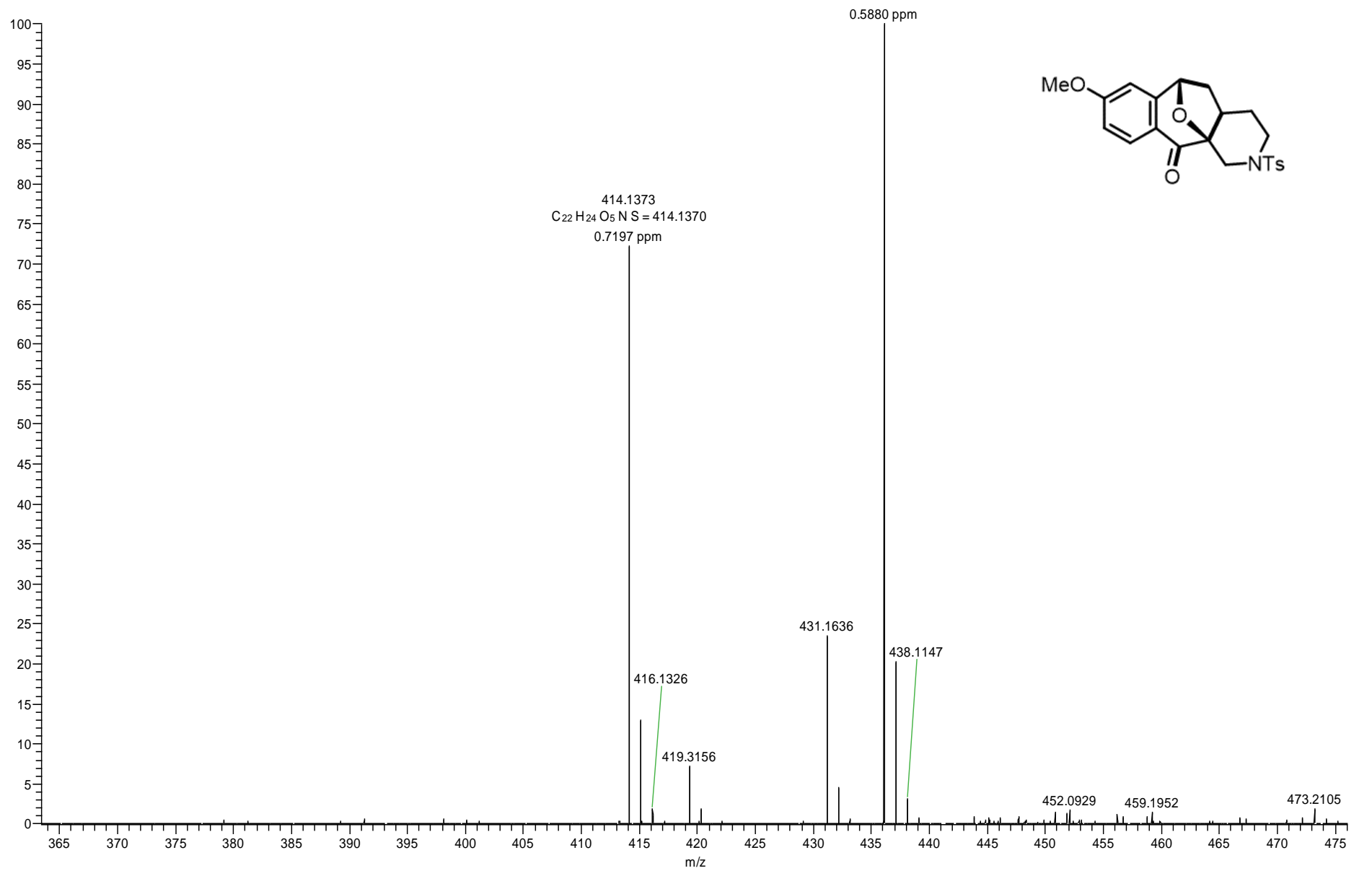

HRMS-ESI Compound $\mathbf{2 h}$ 

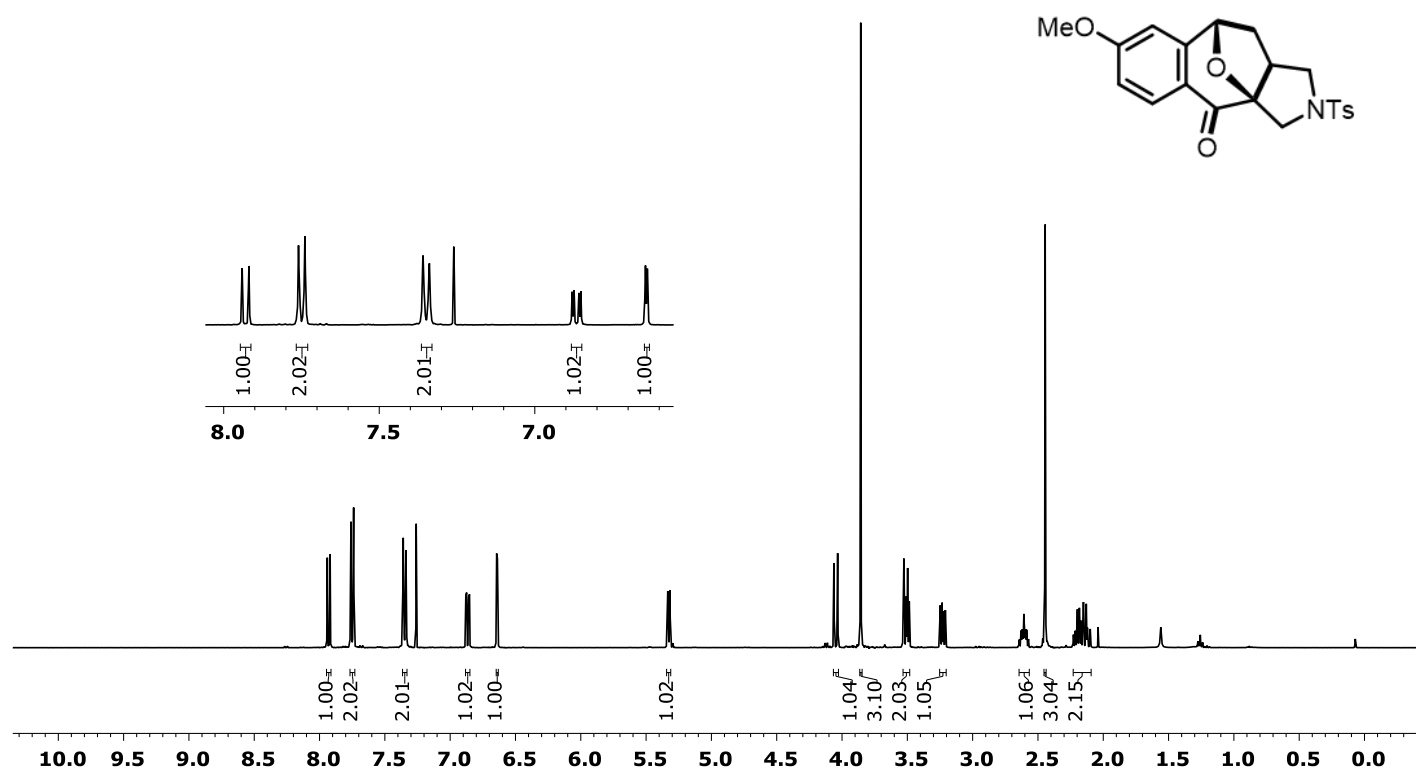

${ }^{1} \mathrm{H}$ NMR Compound $2 \mathbf{i}\left(400 \mathrm{MHz}, \mathrm{CDCl}_{3}\right.$ )

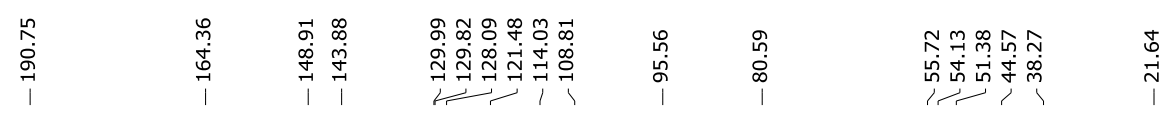
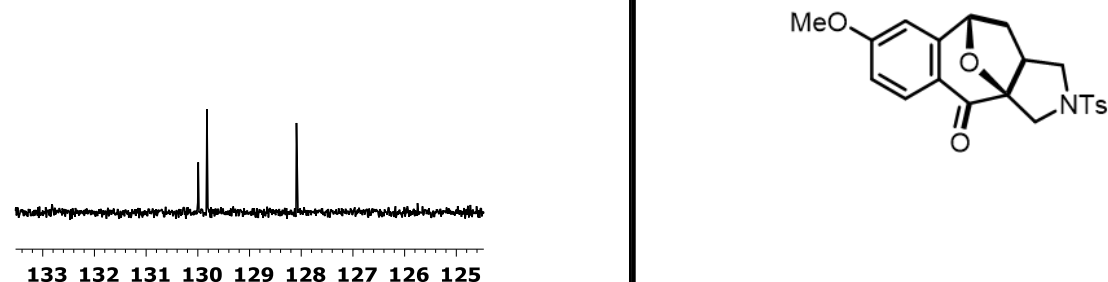

133132131130129128127126125

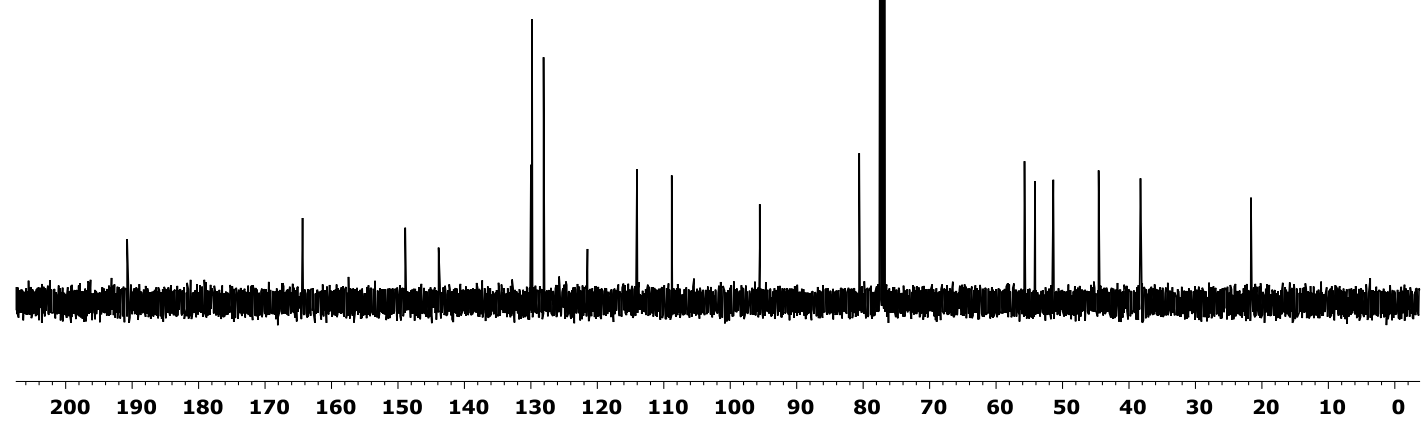

${ }^{13} \mathrm{C}$ NMR Compound $2 \mathbf{i}\left(100 \mathrm{MHz}, \mathrm{CDCl}_{3}\right)$ 
krbta54shr1 \#1 RT: 0.02 AV: 1 NL: 9.71E5 T: FTMS + p ESI Full lock ms [100.00-800.00]

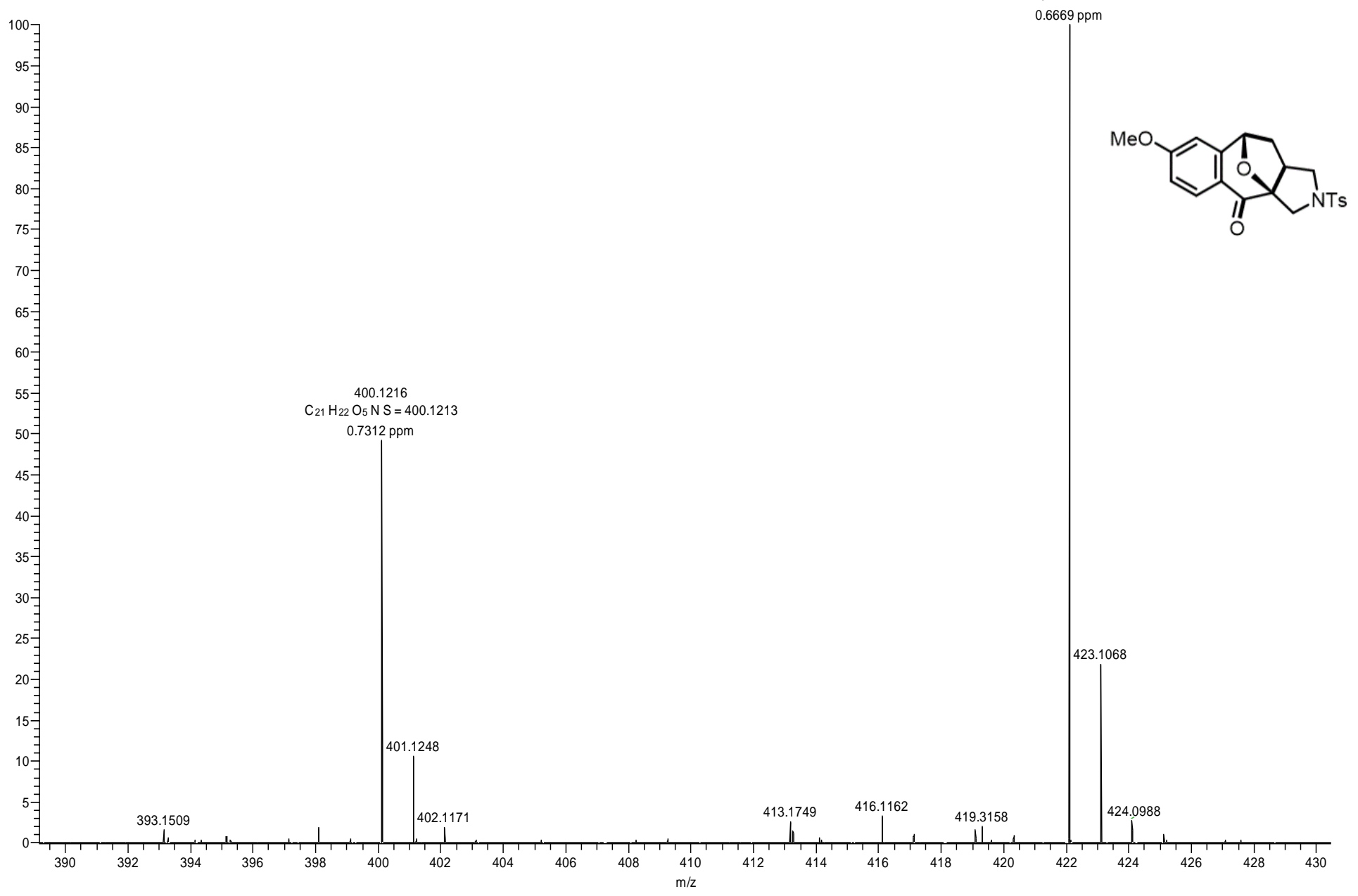

HRMS-ESI Compound 2i 


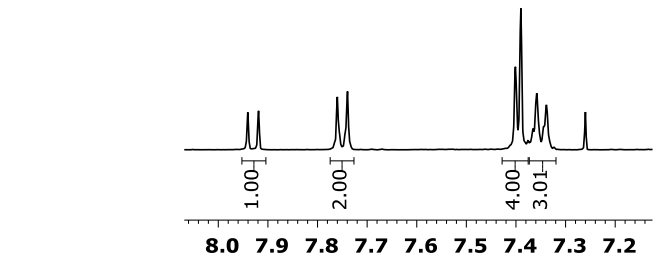

$\mathrm{BnO}$
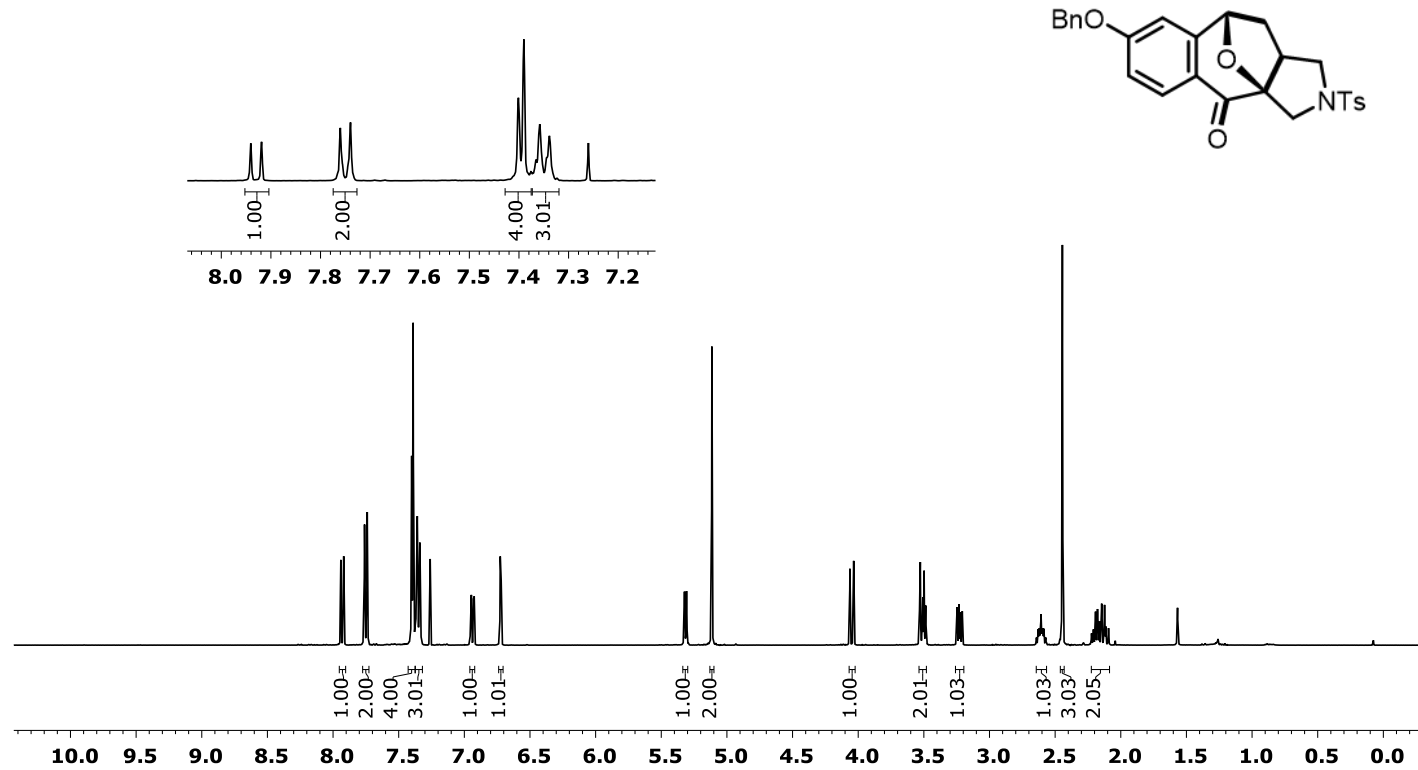

${ }^{1} \mathrm{H}$ NMR Compound 2J (400 MHz, $\left.\mathrm{CDCl}_{3}\right)$

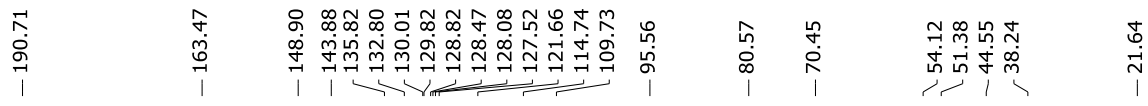

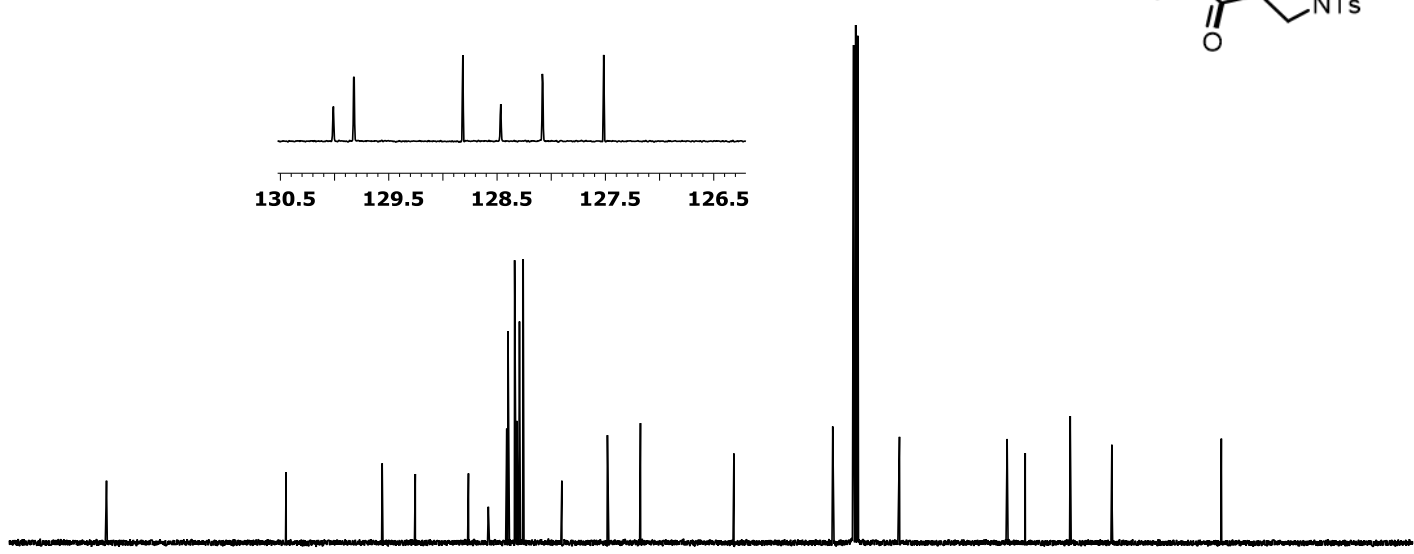

$\begin{array}{lllllllllllllllllllll}200 & 190 & 180 & 170 & 160 & 150 & 140 & 130 & 120 & 110 & 100 & 90 & 80 & 70 & 60 & 50 & 40 & 30 & 20 & 10 & 0\end{array}$

${ }^{13} \mathrm{C}$ NMR Compound 2J (100 MHz, $\left.\mathrm{CDCl}_{3}\right)$ 


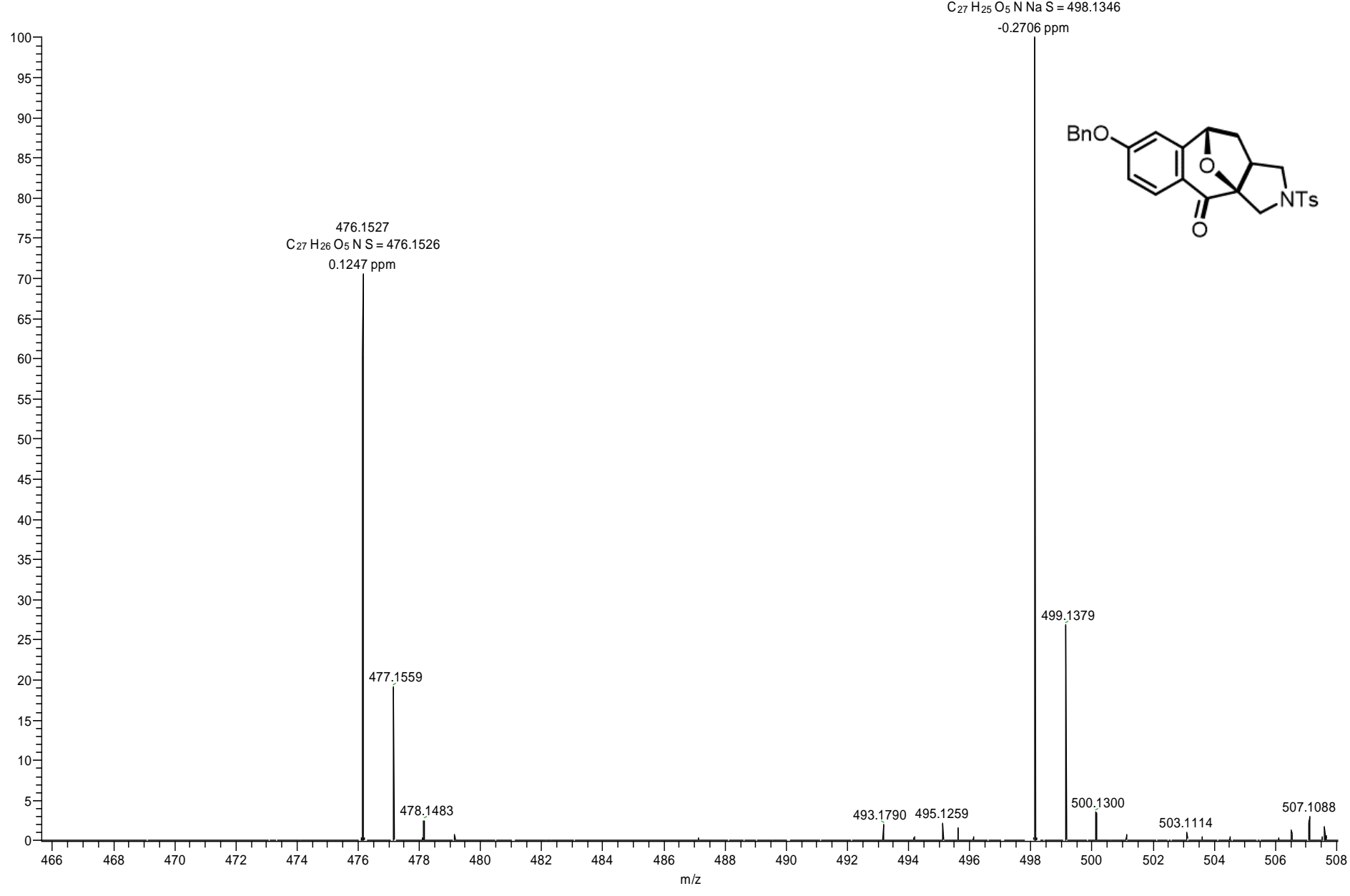

HRMS-ESI Compound 2J 

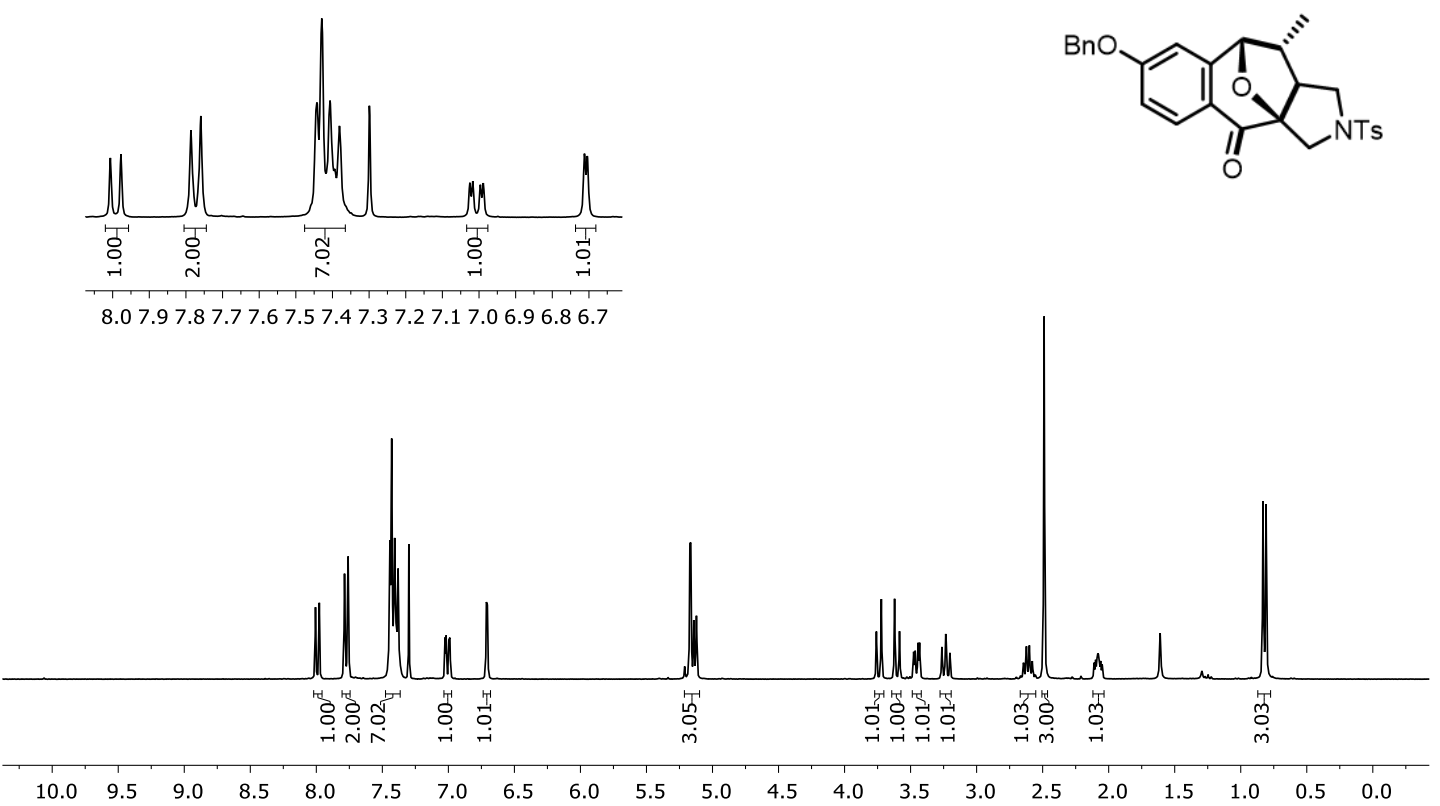

${ }^{1} \mathrm{H}$ NMR Compound 2k (300 MHz, $\left.\mathrm{CDCl}_{3}\right)$

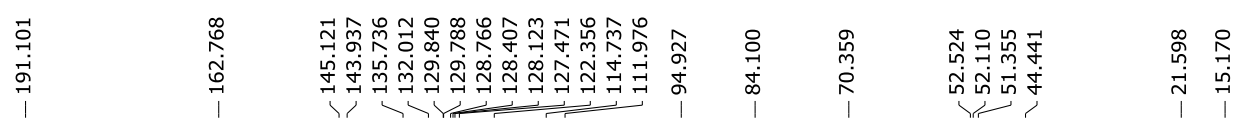
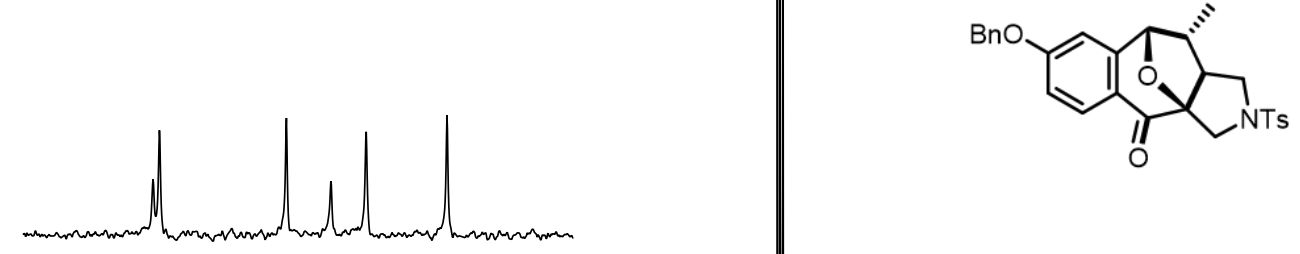

130.5130 .0129 .5129 .0128 .5128 .0127 .5127 .0126 .5

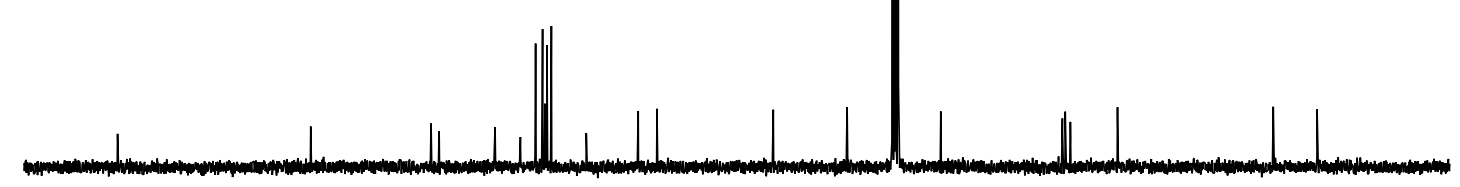

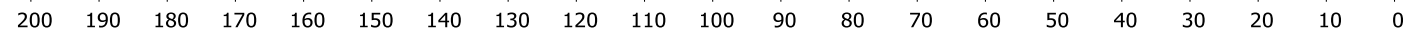

${ }^{13} \mathrm{C}$ NMR Compound $\mathbf{2 k}\left(75 \mathrm{MHz}, \mathrm{CDCl}_{3}\right)$ 


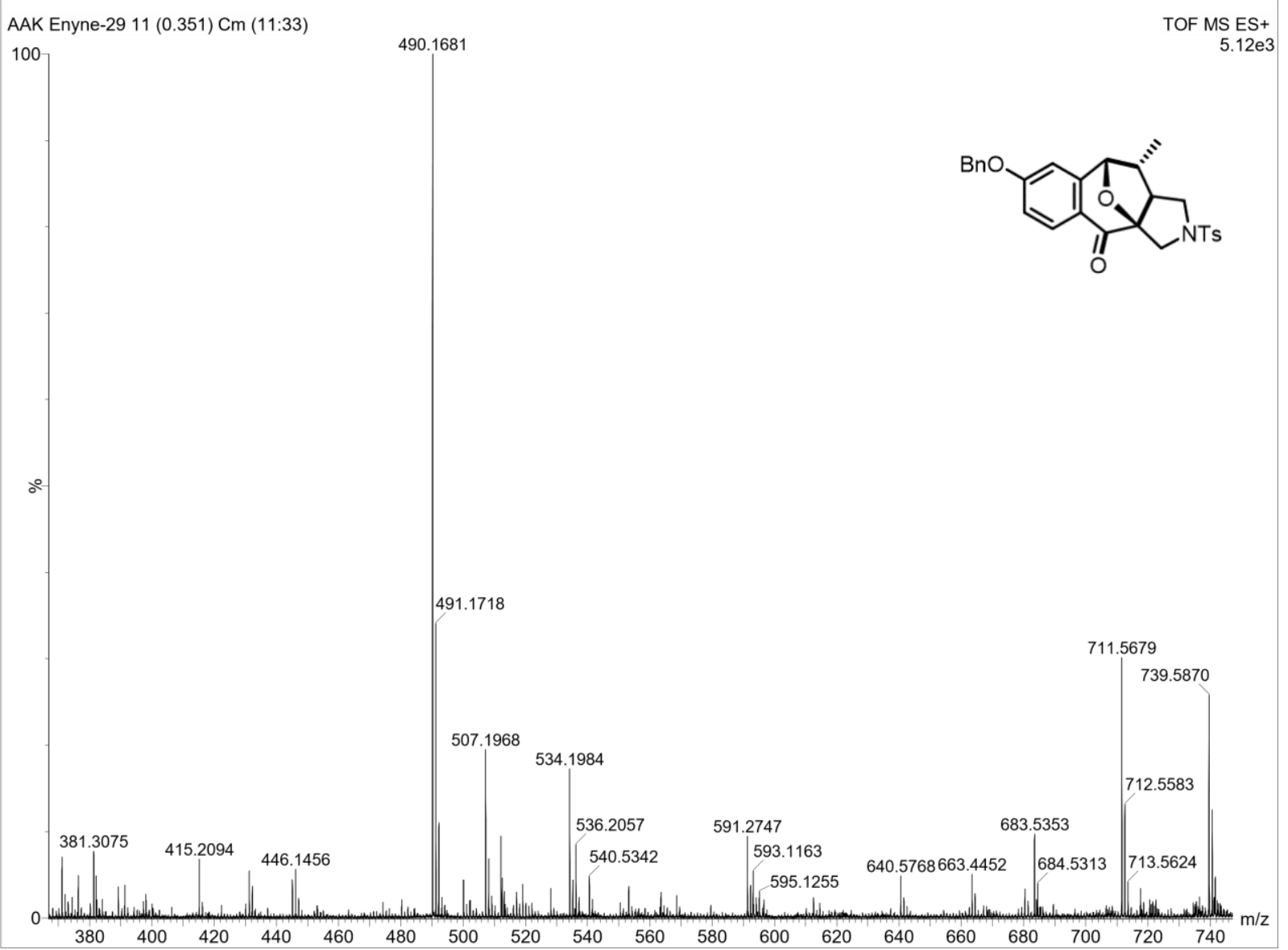

Mass-ESI Compound 2k 

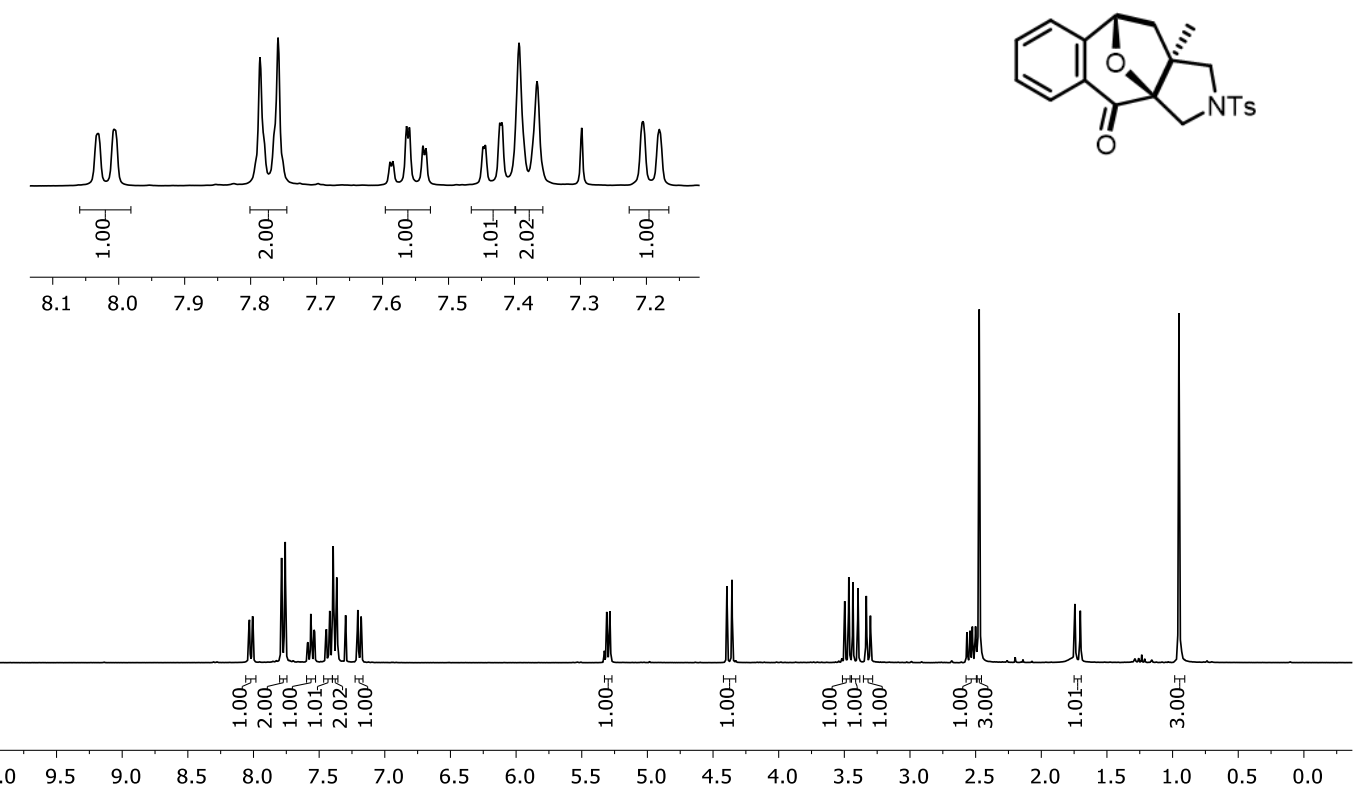

${ }^{1} \mathrm{H}$ NMR Compound $2 \mathrm{l}\left(300 \mathrm{MHz}, \mathrm{CDCl}_{3}\right)$
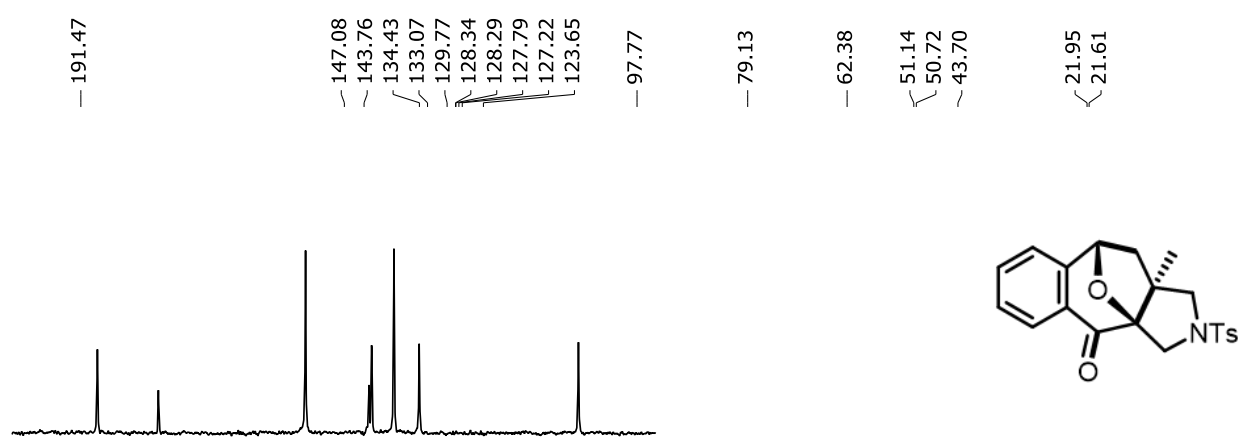

136135134133132131130129128127126125124123122

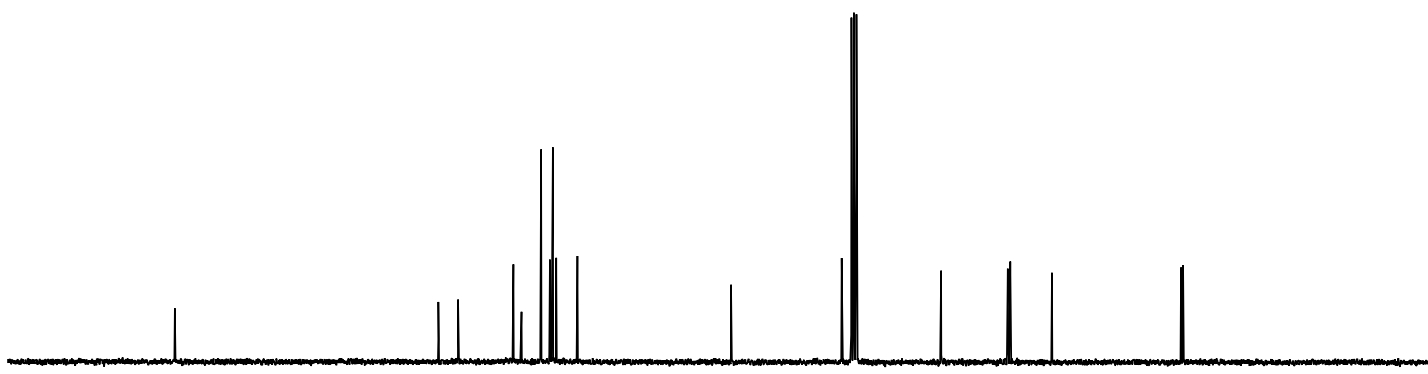

$\begin{array}{lllllllllllllllllllllll}210 & 200 & 190 & 180 & 170 & 160 & 150 & 140 & 130 & 120 & 110 & 100 & 90 & 80 & 70 & 60 & 50 & 40 & 30 & 20 & 10 & 0 & -10\end{array}$

${ }^{13} \mathrm{C}$ NMR Compound $2 \mathrm{l}\left(75 \mathrm{MHz}, \mathrm{CDCl}_{3}\right)$ 


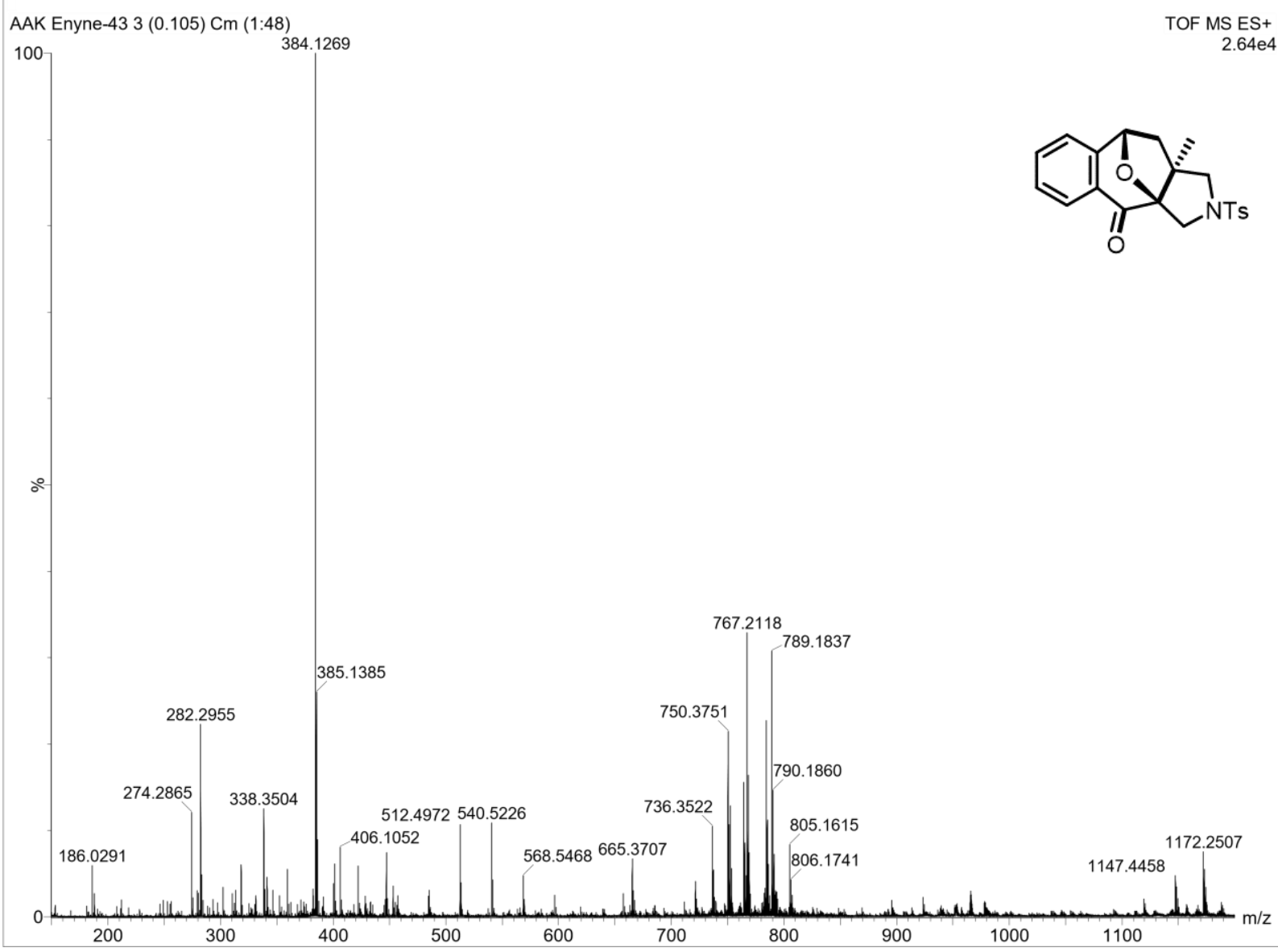

Mass-ESI Compound 2l 

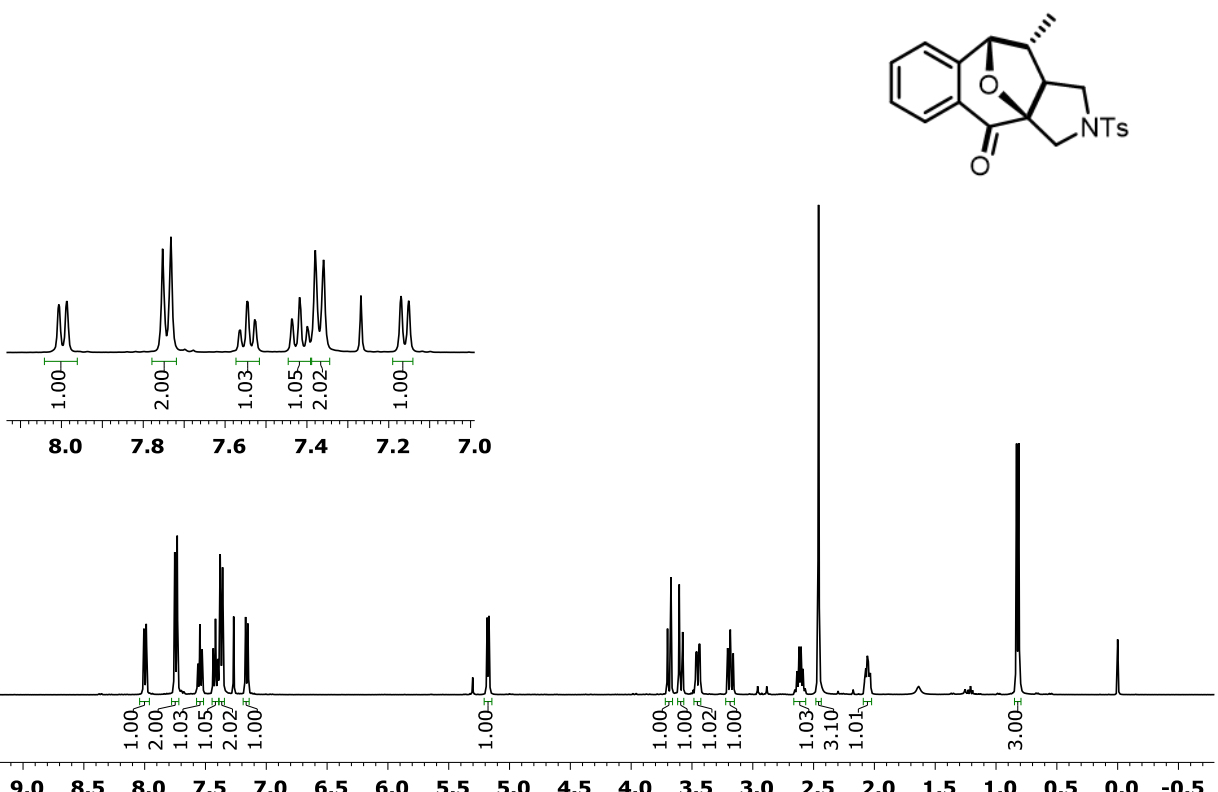

${ }^{1} \mathrm{H}$ NMR Compound $2 \mathrm{~m}\left(400 \mathrm{MHz}, \mathrm{CDCl}_{3}\right)$

$\underset{\substack{0 \\ \stackrel{1}{1}}}{\substack{1 \\ j}}$
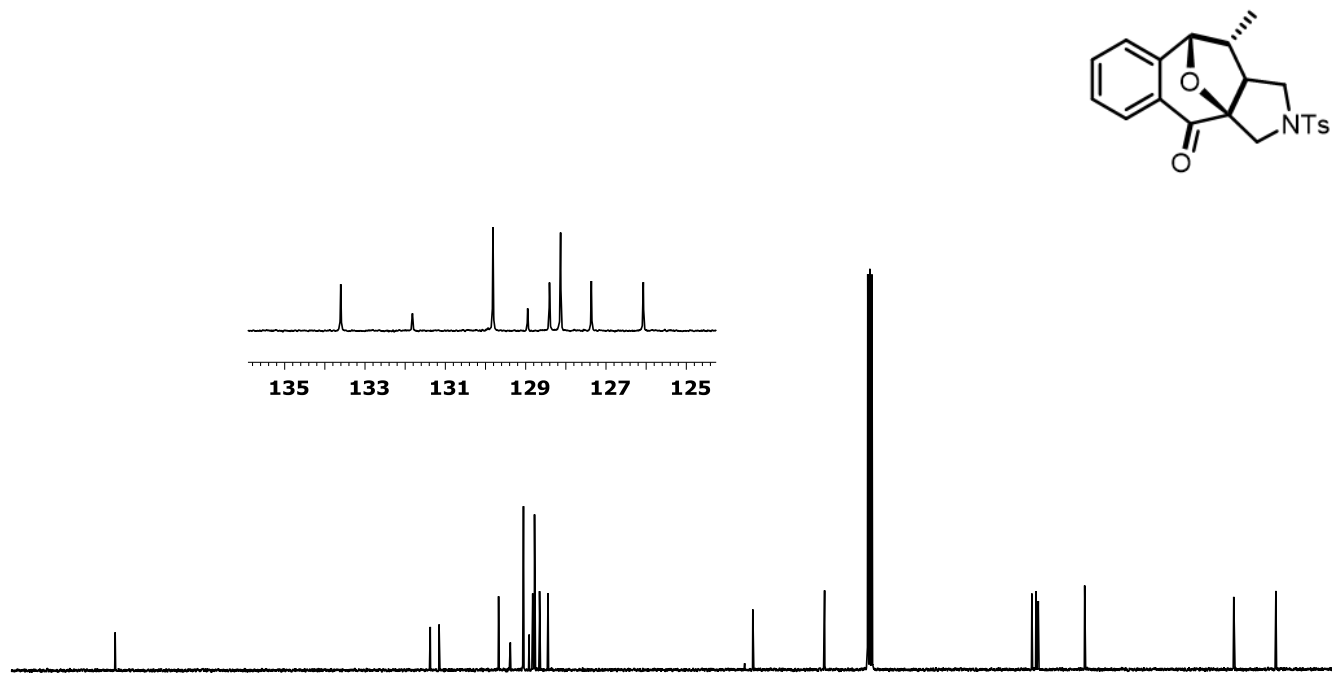

$\begin{array}{lllllllllllllllllllll}200 & 190 & 180 & 170 & 160 & 150 & 140 & 130 & 120 & 110 & 100 & 90 & 80 & 70 & 60 & 50 & 40 & 30 & 20 & 10 & 0\end{array}$

${ }^{13} \mathrm{C}$ NMR Compound $\mathbf{2 m}\left(100 \mathrm{MHz}, \mathrm{CDCl}_{3}\right)$ 


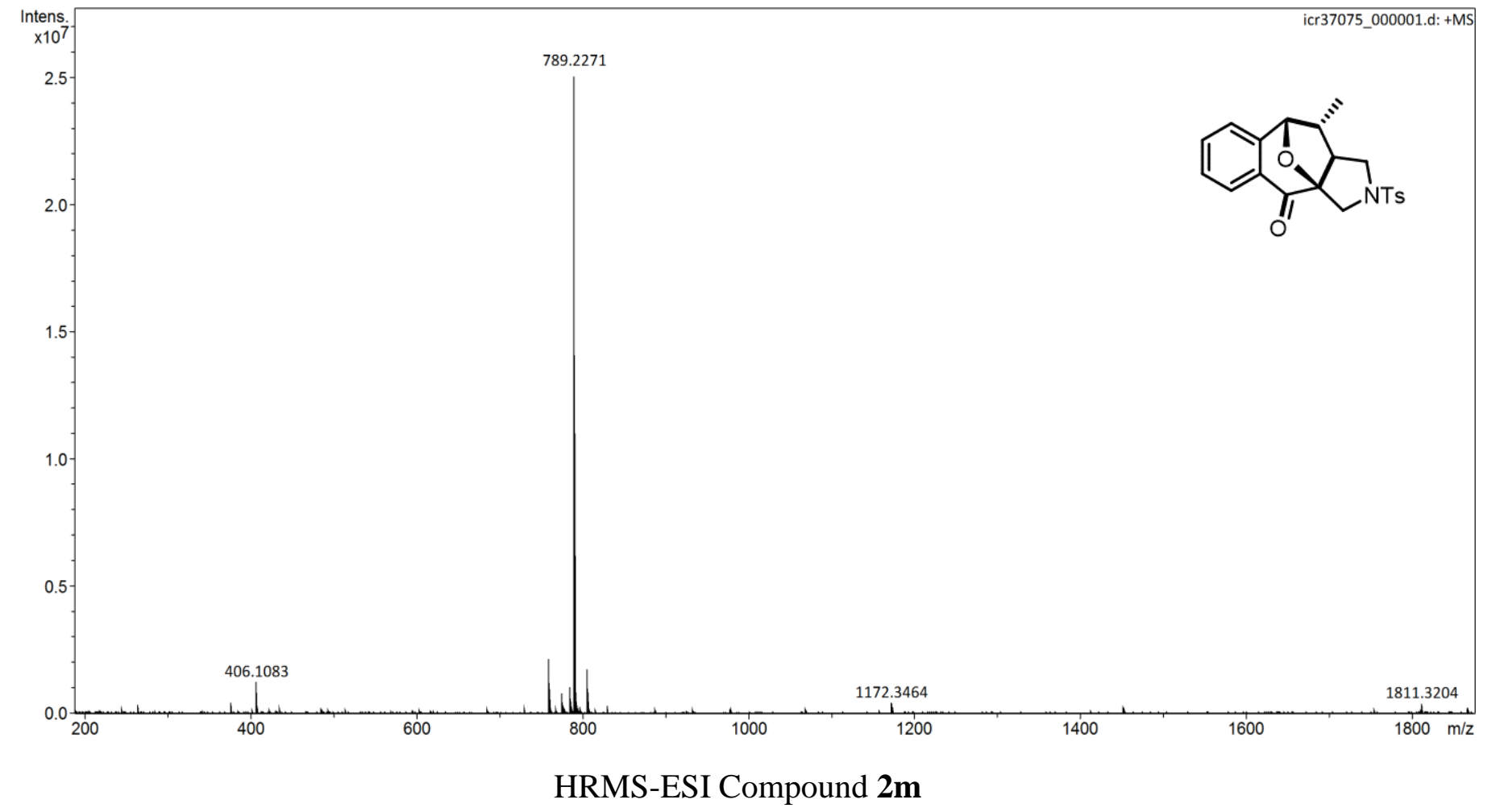

$\begin{array}{ccrrrrrr}\text { Meas. } \mathrm{m} / \mathrm{z} & \text { Ion Formula } & \mathrm{m} / \mathrm{z} & \mathrm{err}[\mathrm{ppm}] & \mathrm{mSigma} & \mathrm{rdb} & \mathrm{e}^{-} \text {Conf } & \text { N-Rule } \\ 406.1083 & \mathrm{C} 21 \mathrm{H} 21 \mathrm{NNaO} 4 \mathrm{~S} & 406.1083 & 0.1 & 11.0 & 11.5 & \text { even } & \text { ok } \\ & \mathrm{C} 20 \mathrm{H} 22 \mathrm{O} 7 \mathrm{~S} & 406.1081 & -0.6 & 14.9 & 10.0 & \text { odd } & \text { ok } \\ & \mathrm{C} 27 \mathrm{H} 15 \mathrm{~N} 2 \mathrm{NaO} & 406.1077 & -1.6 & 21.6 & 21.0 & \text { odd } & \text { ok } \\ & \mathrm{C} 27 \mathrm{H} 12 \mathrm{~N} 5 & 406.1087 & 1.0 & 22.9 & 24.5 & \text { even } & \text { ok } \\ 789.2271 & \mathrm{C} 19 \mathrm{H} 16 \mathrm{~N} 7 \mathrm{O} 2 \mathrm{~S} & 406.1081 & -0.6 & 113.3 & 15.5 & \text { even } & \text { ok } \\ & \mathrm{C} 42 \mathrm{H} 42 \mathrm{~N} 2 \mathrm{NaO} 8 \mathrm{~S} 2 & 789.2275 & 0.4 & 26.0 & 22.5 & \text { even } & \text { ok } \\ & \text { C42H39N5O7S2 } & 789.2285 & 1.8 & 28.9 & 26.0 & \text { odd } & \text { ok } \\ & \mathrm{C} 42 \mathrm{H} 34 \mathrm{~N} 6 \mathrm{NaO} 9 & 789.2279 & 1.0 & 34.5 & 28.5 & \text { even } & \text { ok }\end{array}$



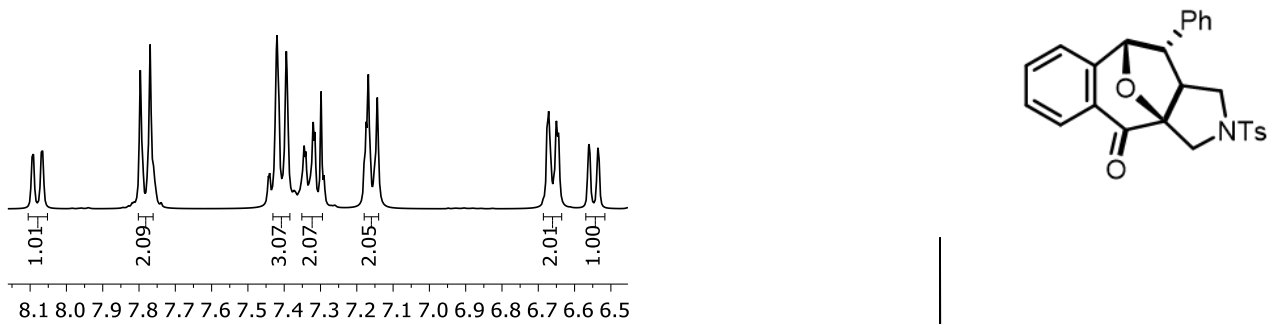

8.1 8.0 7.9 7.8 7.7 7.6 7.5 7.4 7.3 7.2 7.17.06.96.8 6.7 6.6 6.5

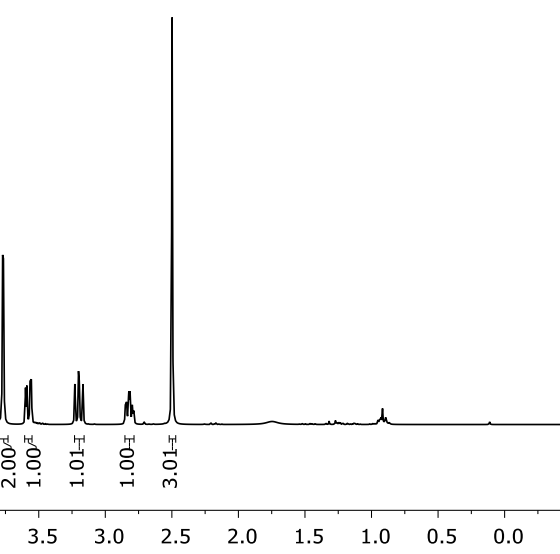

${ }^{1} \mathrm{H}$ NMR Compound $2 \mathbf{n}\left(300 \mathrm{MHz}, \mathrm{CDCl}_{3}\right)$

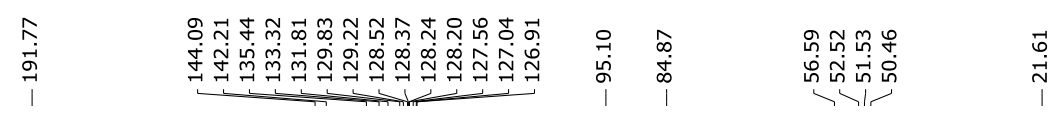
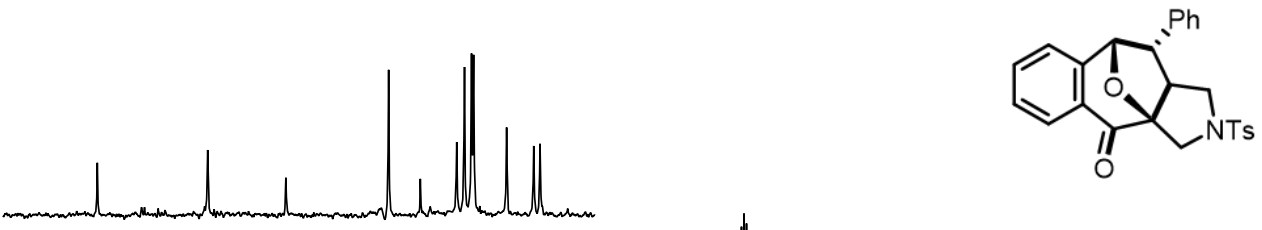

$\begin{array}{llllllllllll}137 & 136 & 135 & 134 & 133 & 132 & 131 & 130 & 129 & 128 & 127 & 126\end{array}$

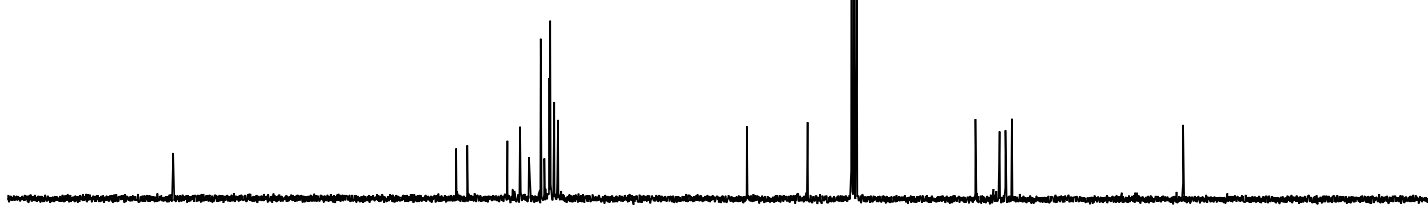

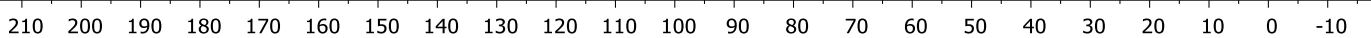

${ }^{13} \mathrm{C}$ NMR Compound 2n $\left(75 \mathrm{MHz}, \mathrm{CDCl}_{3}\right)$ 


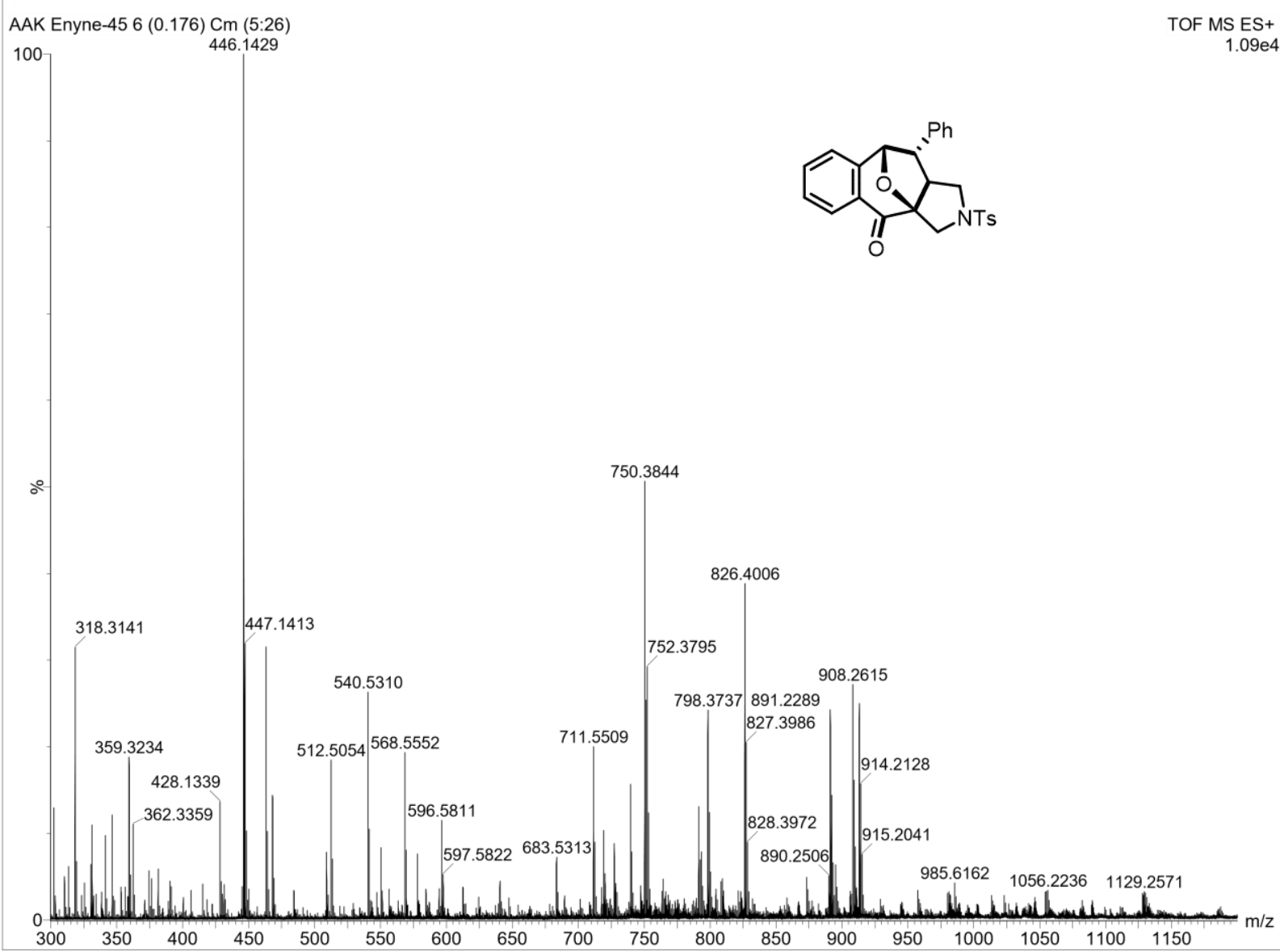

Mass-ESI Compound 2n 

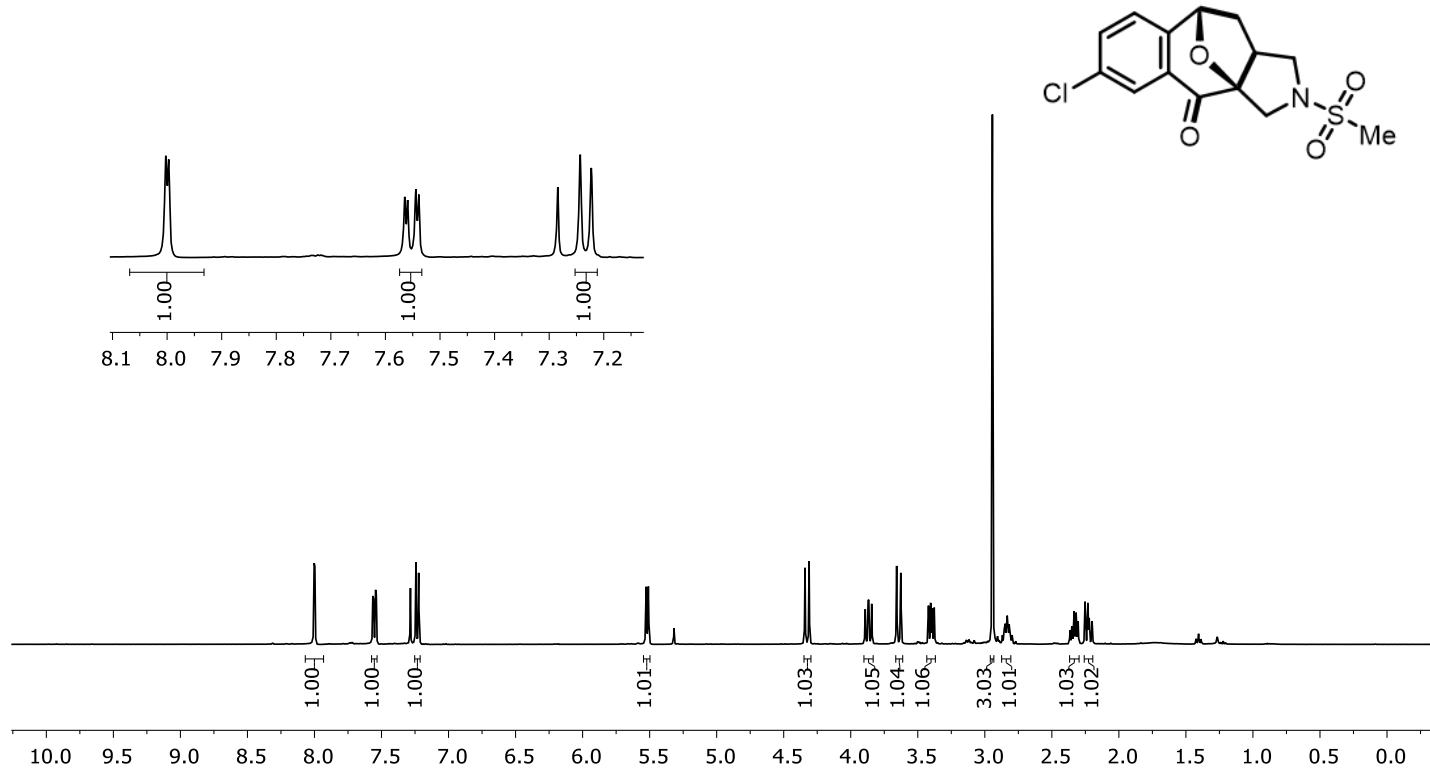

${ }^{1} \mathrm{H}$ NMR Compound $20\left(400 \mathrm{MHz}, \mathrm{CDCl}_{3}\right)$

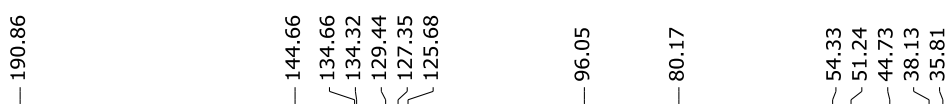
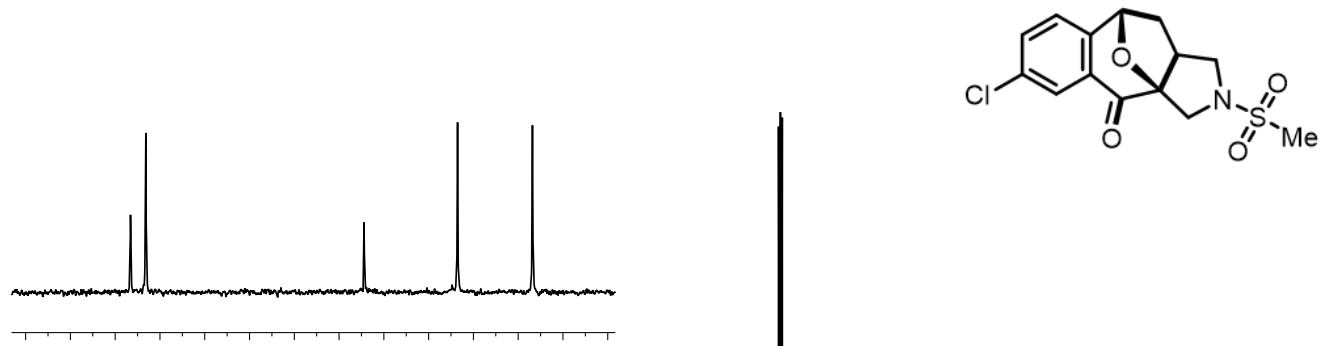

137136135134133132131130129128127126125124

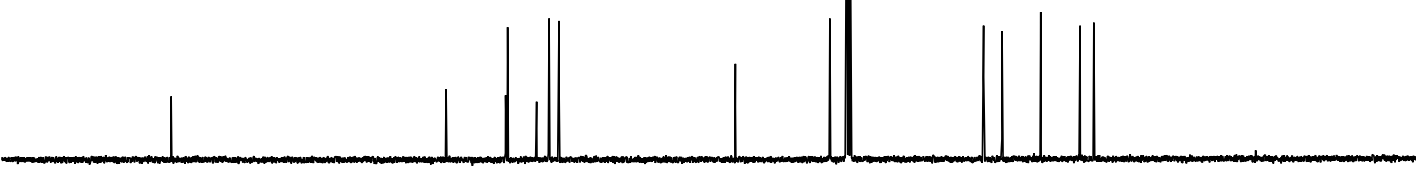

$\begin{array}{lllllllllllllllllllllll}210 & 200 & 190 & 180 & 170 & 160 & 150 & 140 & 130 & 120 & 110 & 100 & 90 & 80 & 70 & 60 & 50 & 40 & 30 & 20 & 10 & 0 & -10\end{array}$

${ }^{13} \mathrm{C}$ NMR Compound $20\left(100 \mathrm{MHz}, \mathrm{CDCl}_{3}\right)$ 


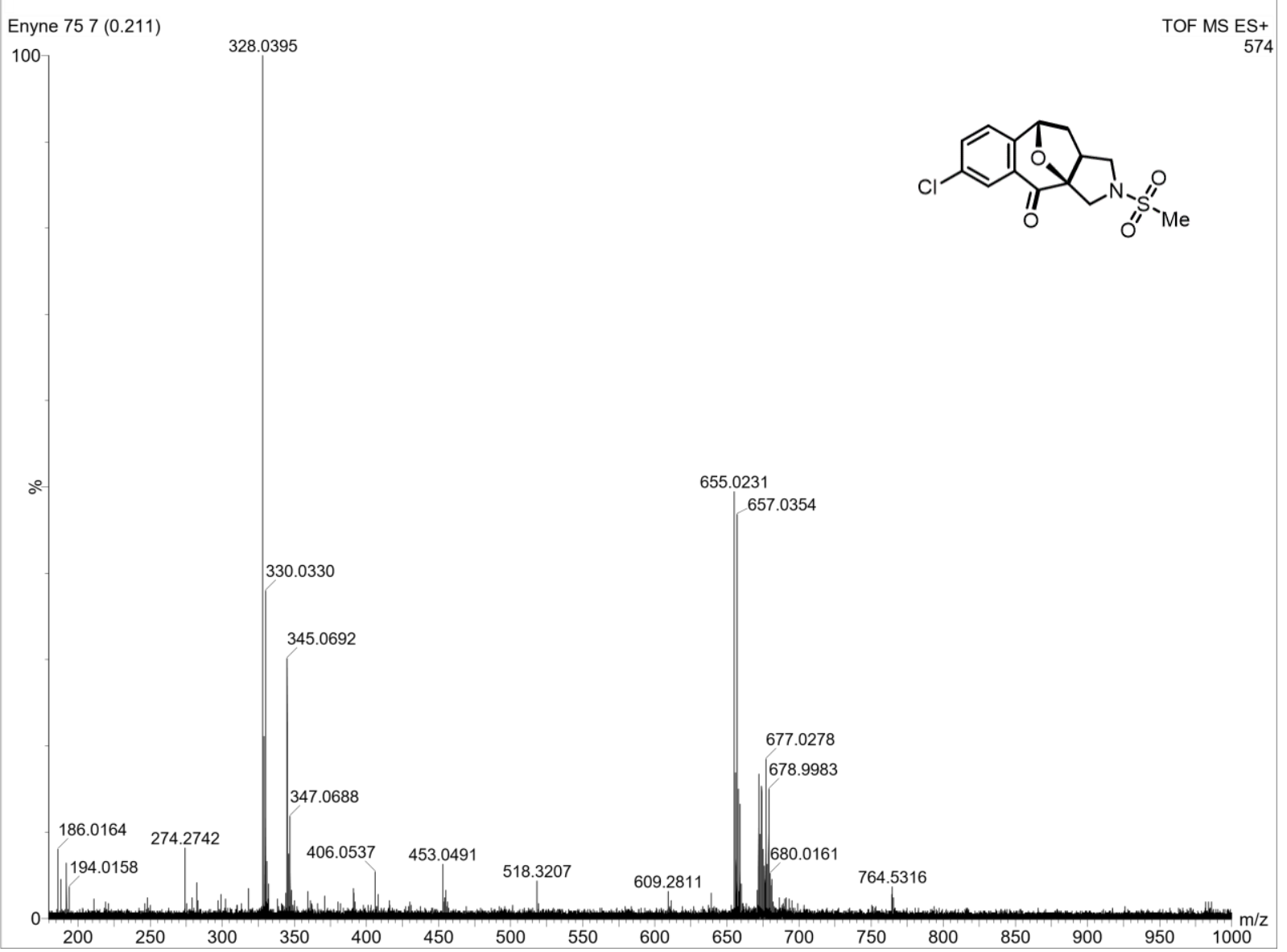

Mass-ESI Compound 20 

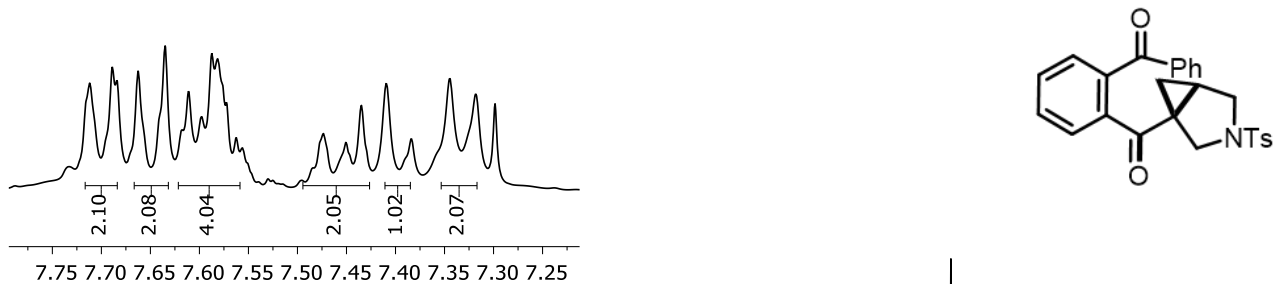

7.75 7.70 7.65 7.60 7.55 7.507.457.407.357.307.25

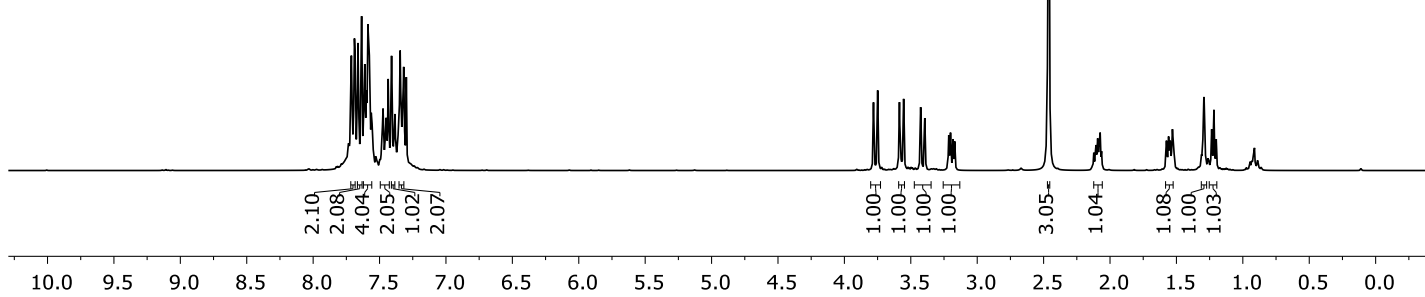

${ }^{1} \mathrm{H}$ NMR Compound $4 \mathbf{a}\left(300 \mathrm{MHz}, \mathrm{CDCl}_{3}\right)$

유ำ 궁

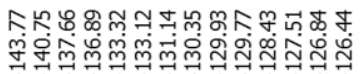
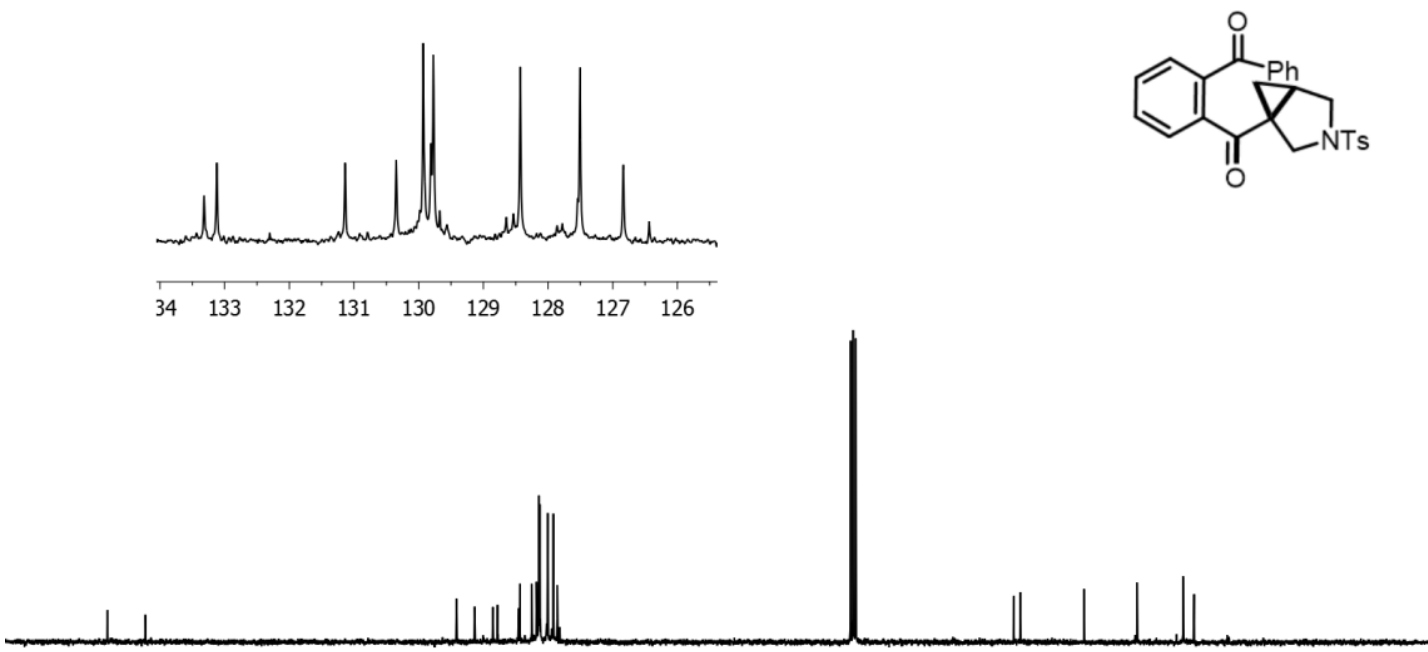

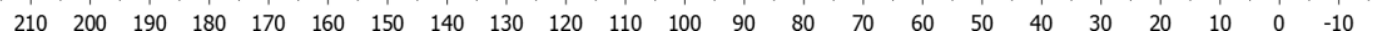

${ }^{13} \mathrm{C}$ NMR Compound $4 \mathbf{a}\left(75 \mathrm{MHz}, \mathrm{CDCl}_{3}\right)$ 


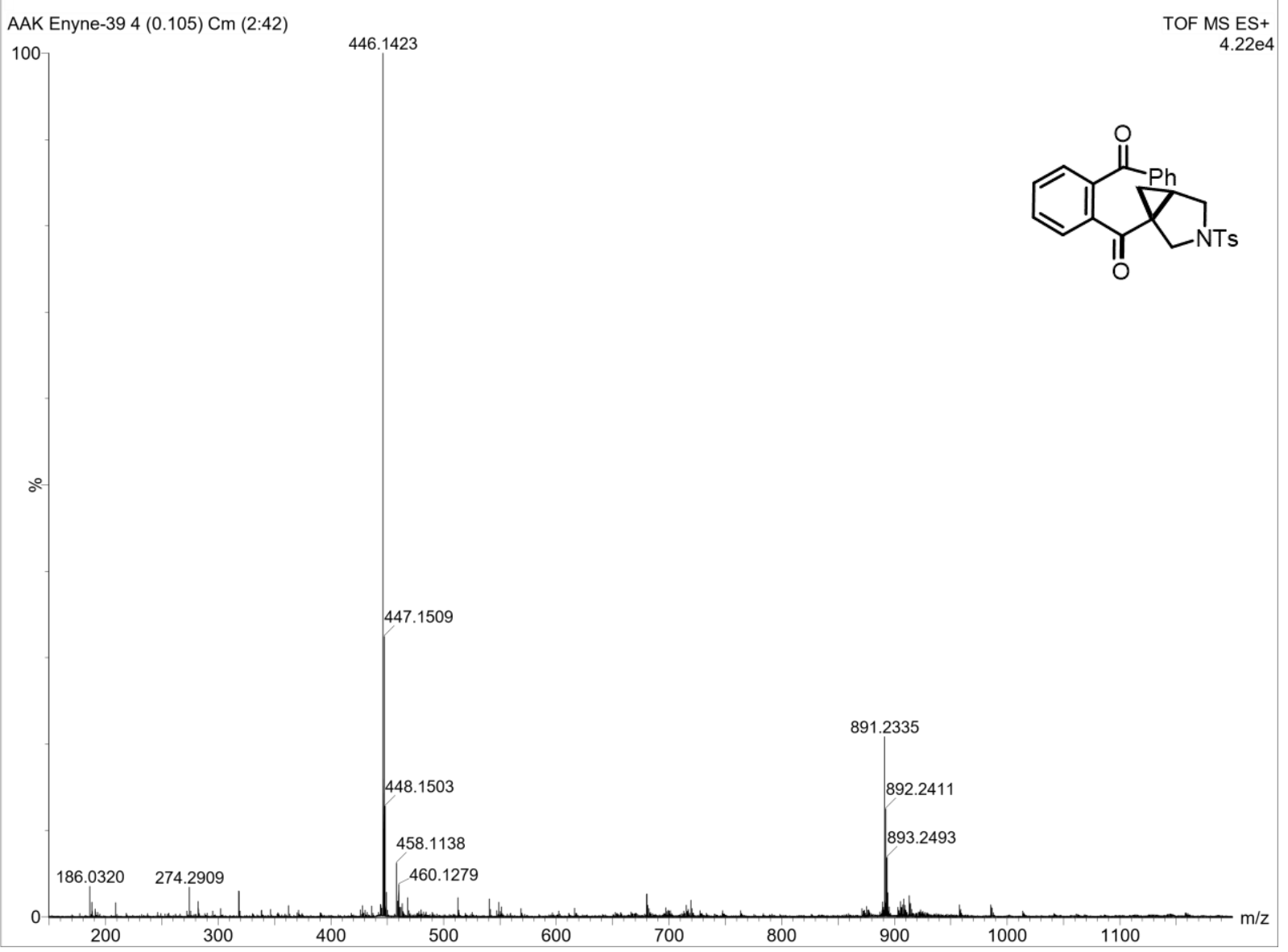

Mass-ESI Compound 4a 

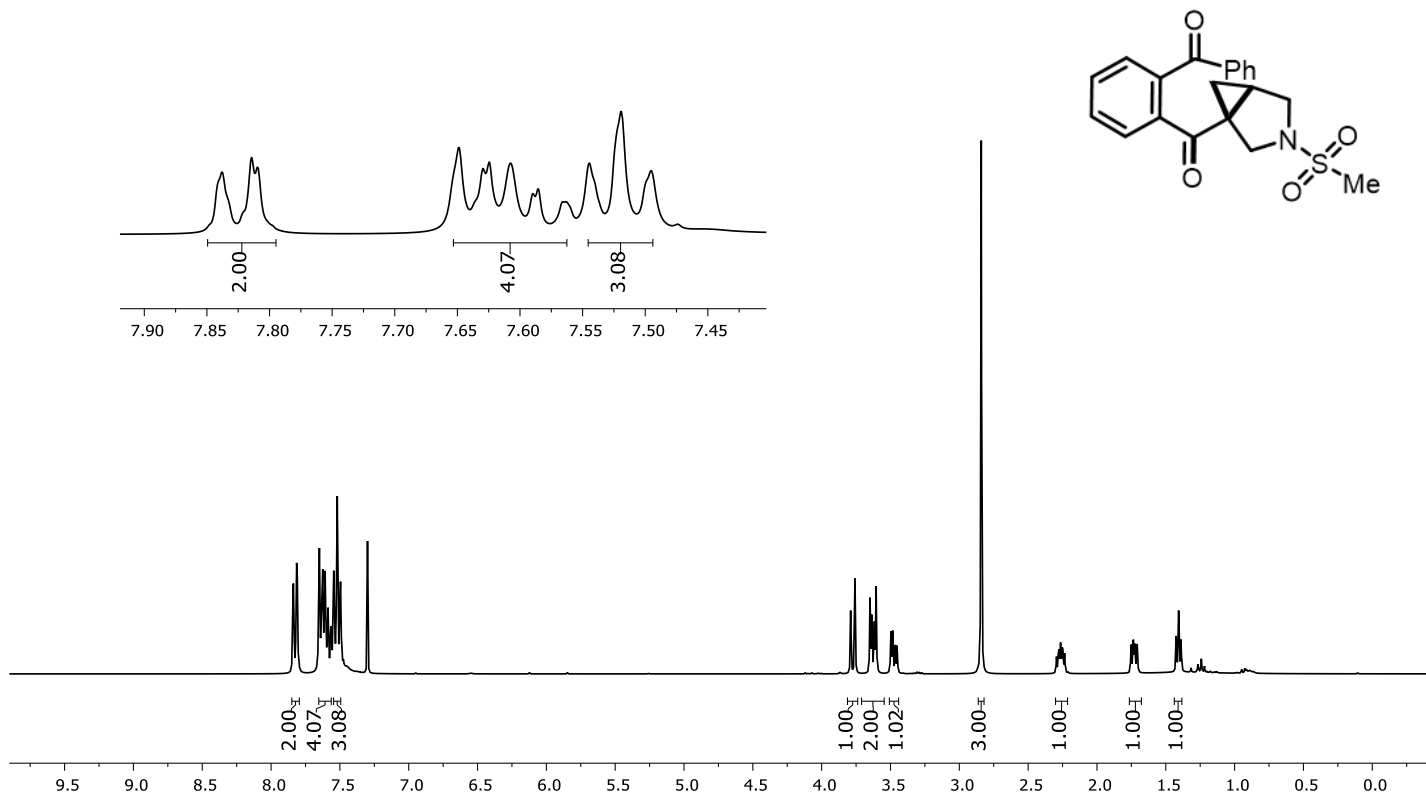

${ }^{1} \mathrm{H}$ NMR Compound $\mathbf{4 b}\left(300 \mathrm{MHz}, \mathrm{CDCl}_{3}\right)$

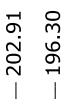

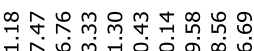

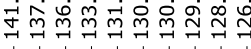

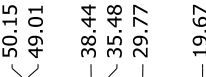
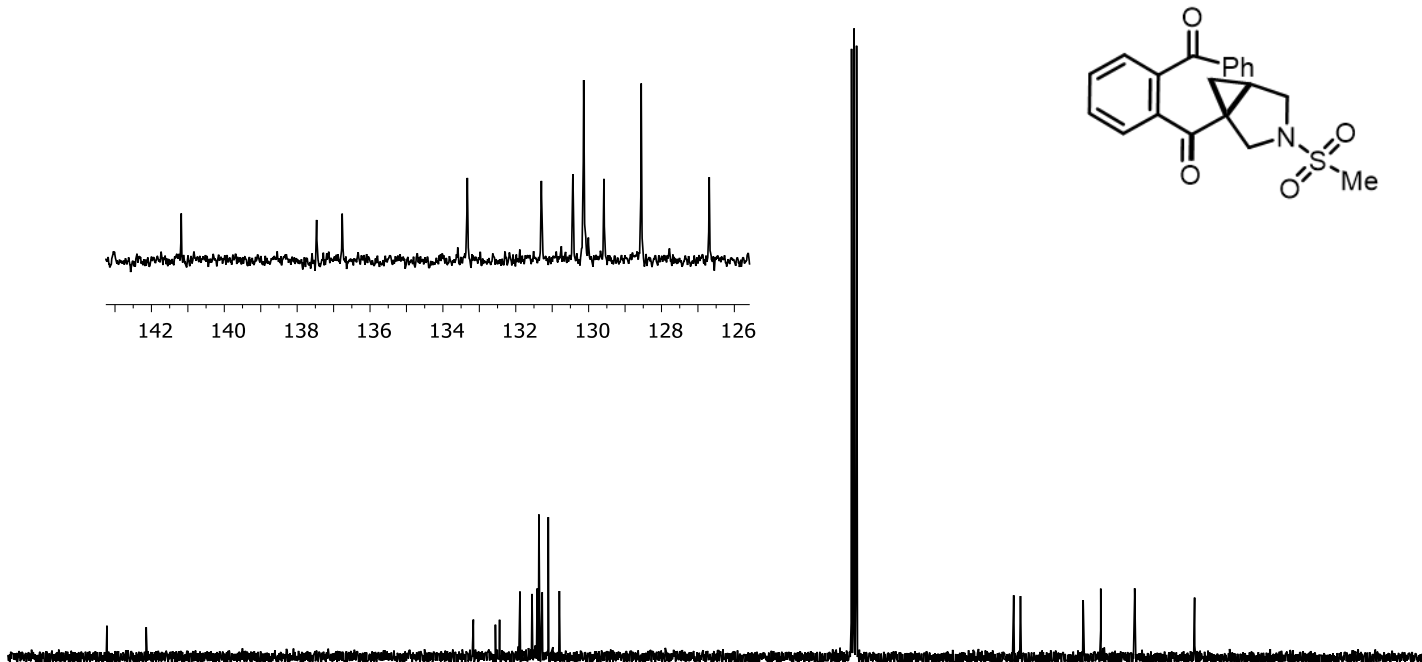

$\begin{array}{lllllllllllllllllllllll}210 & 200 & 190 & 180 & 170 & 160 & 150 & 140 & 130 & 120 & 110 & 100 & 90 & 80 & 70 & 60 & 50 & 40 & 30 & 20 & 10 & 0 & -10\end{array}$

${ }^{13} \mathrm{C}$ NMR Compound $4 \mathbf{b}\left(75 \mathrm{MHz}, \mathrm{CDCl}_{3}\right)$ 


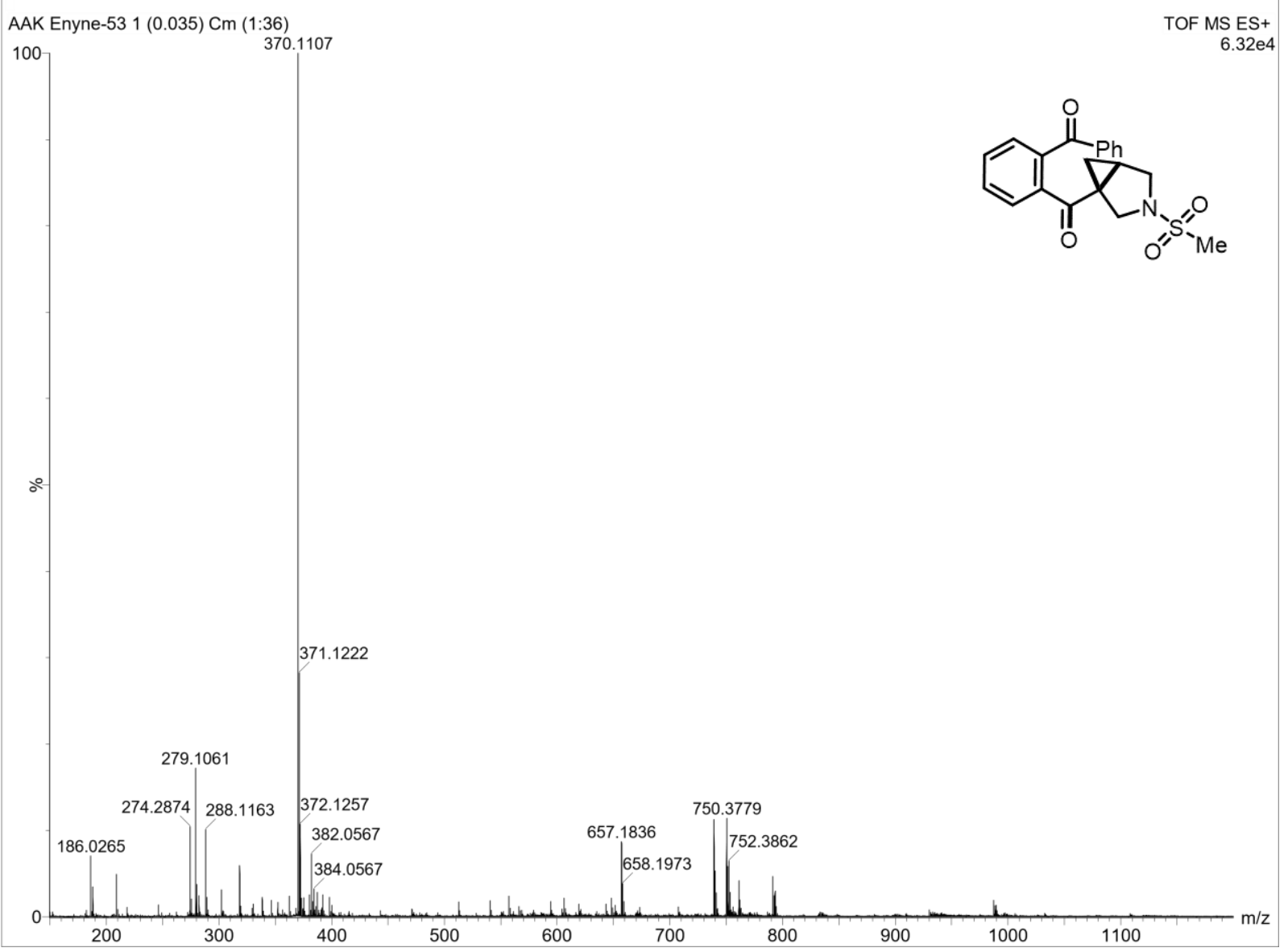

Mass-ESI Compound 4b 

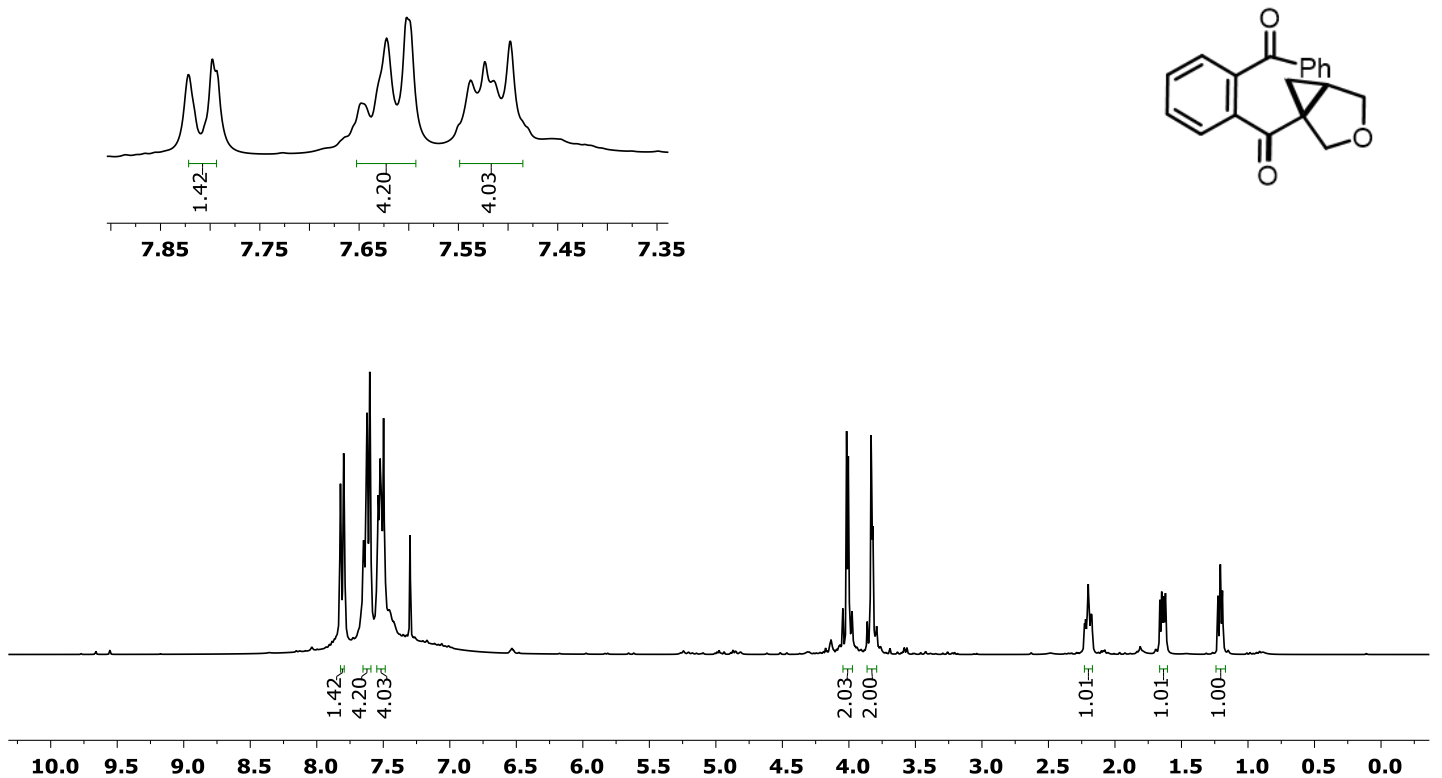

${ }^{1} \mathrm{H}$ NMR Compound $4 \mathbf{c}\left(300 \mathrm{MHz}, \mathrm{CDCl}_{3}\right)$

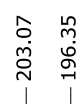
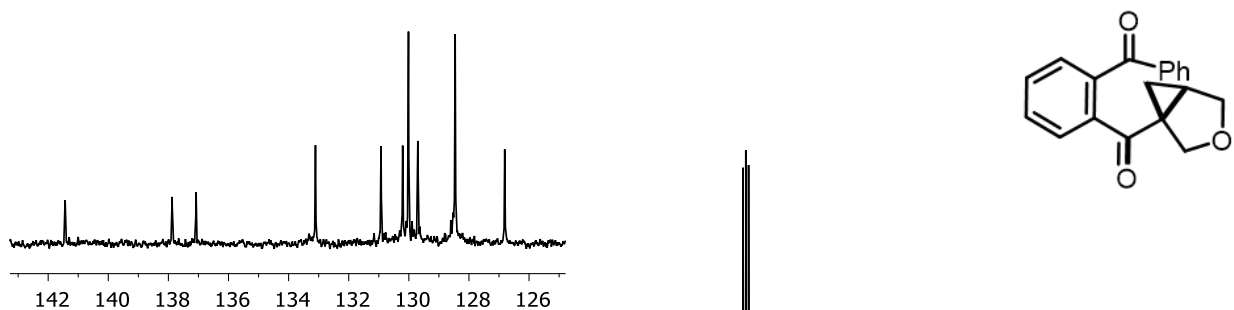

$\begin{array}{lllllllll}142 & 140 & 138 & 136 & 134 & 132 & 130 & 128 & 126\end{array}$

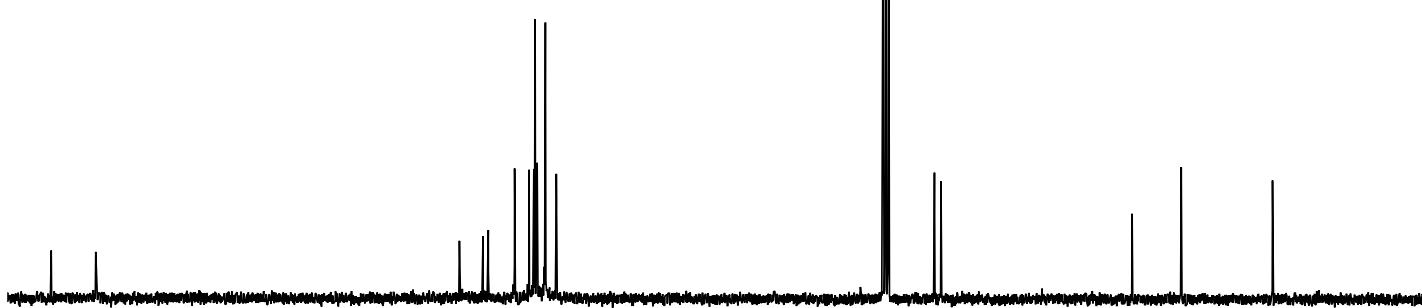

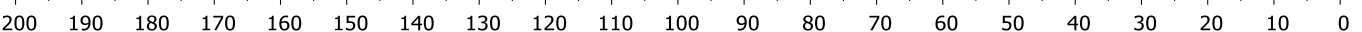

${ }^{13} \mathrm{C}$ NMR Compound $4 \mathbf{c}\left(75 \mathrm{MHz}, \mathrm{CDCl}_{3}\right)$ 


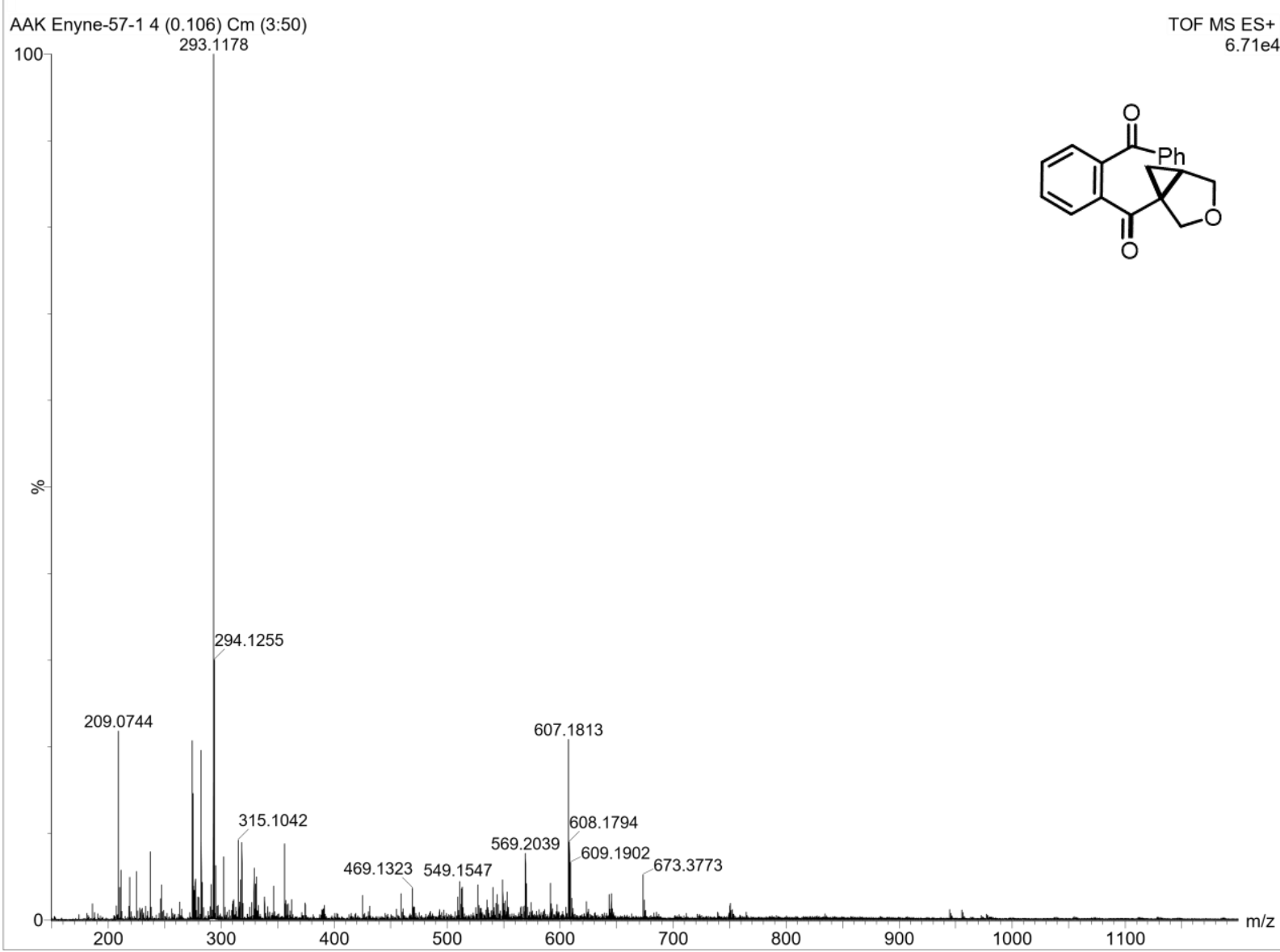

Mass-ESI Compound 4c 

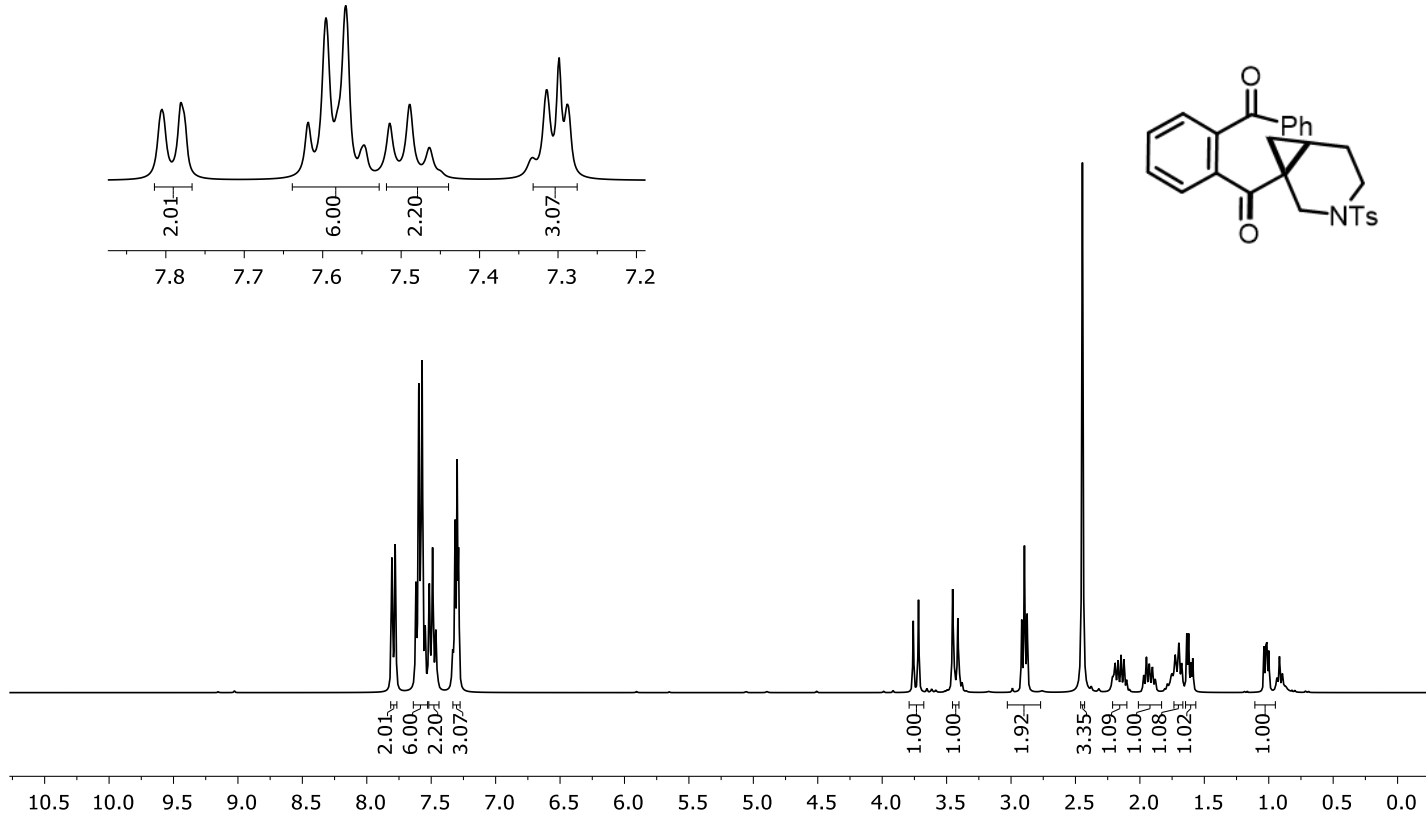

${ }^{1} \mathrm{H}$ NMR Compound $4 \mathbf{d}\left(300 \mathrm{MHz}, \mathrm{CDCl}_{3}\right)$

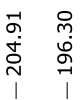
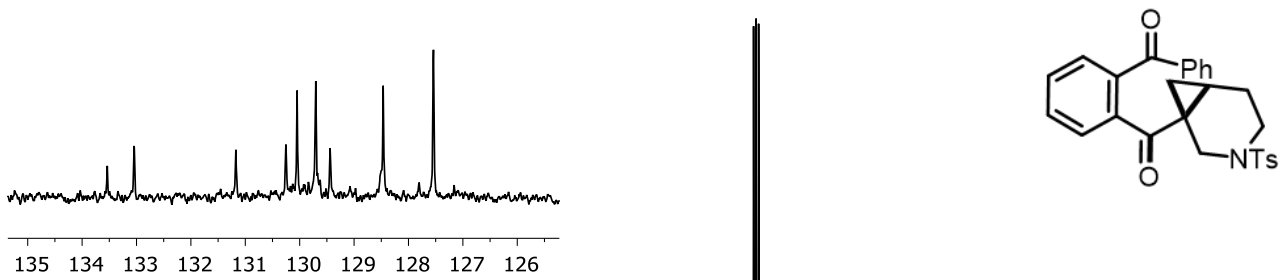

$\begin{array}{lllllllllll}135 & 134 & 133 & 132 & 131 & 130 & 129 & 128 & 127 & 126\end{array}$
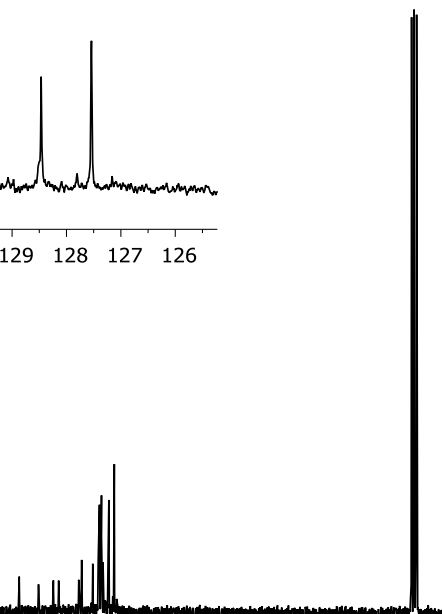

$\begin{array}{llllllllllllllllllllllll}210 & 200 & 190 & 180 & 170 & 160 & 150 & 140 & 130 & 120 & 110 & 100 & 90 & 80 & 70 & 60 & 50 & 40 & 30 & 20 & 10 & 0 & -10\end{array}$

${ }^{13} \mathrm{C}$ NMR Compound $4 \mathbf{d}\left(75 \mathrm{MHz}, \mathrm{CDCl}_{3}\right)$ 


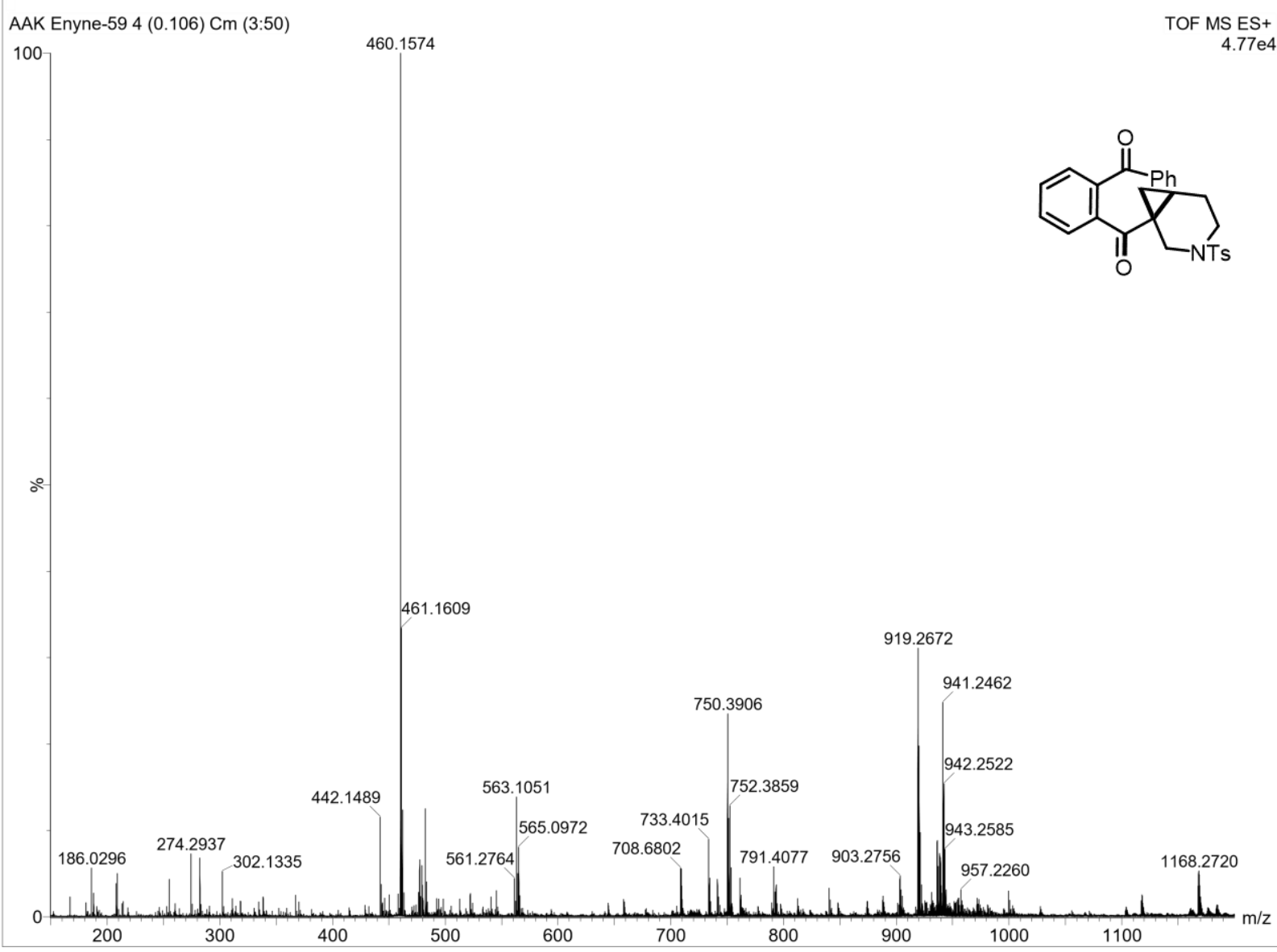

Mass-ESI Compound 4d 

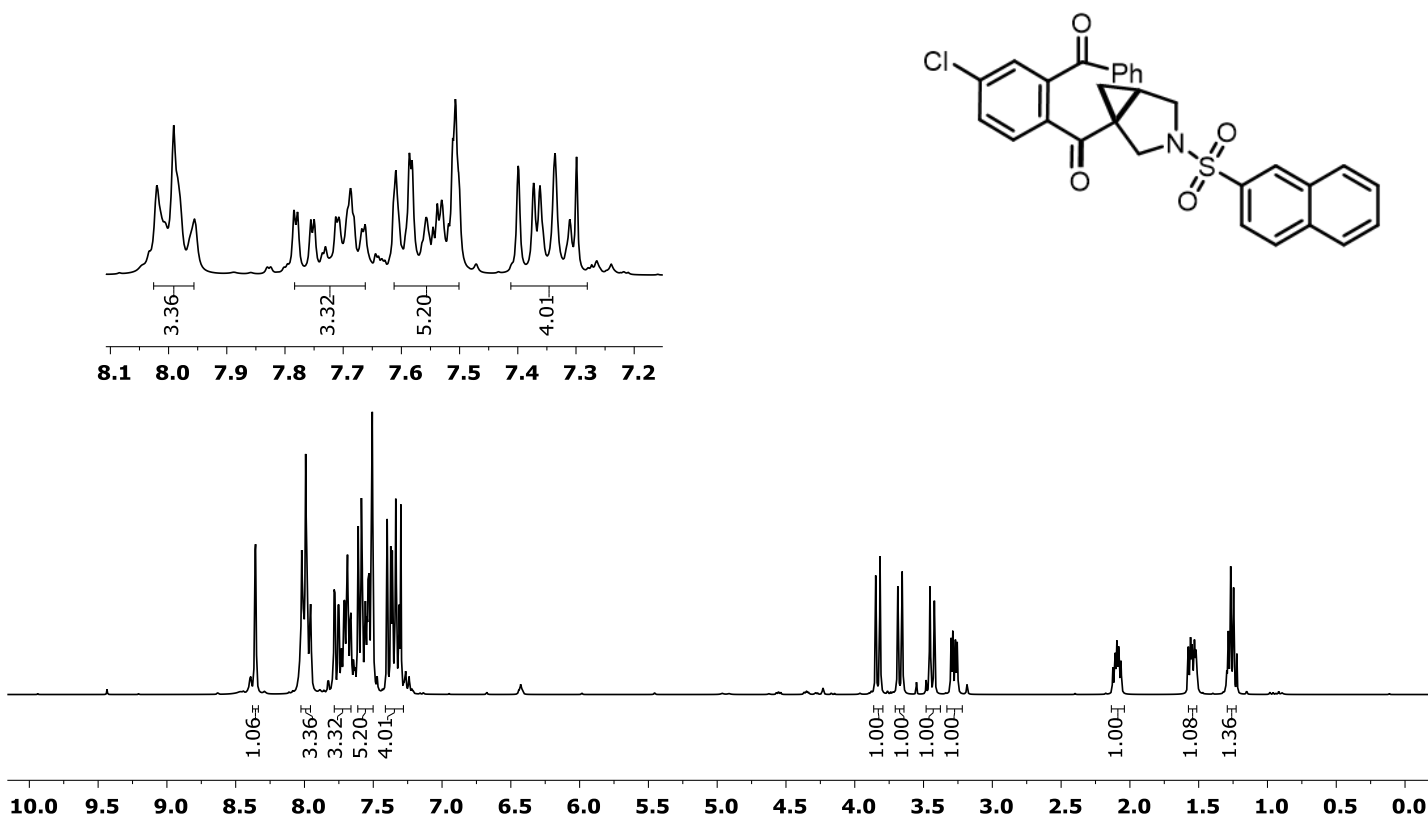

${ }^{1} \mathrm{H}$ NMR Compound $4 \mathrm{e}\left(300 \mathrm{MHz}, \mathrm{CDCl}_{3}\right)$

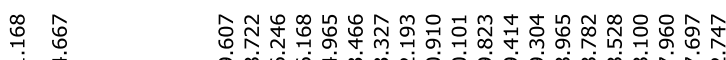

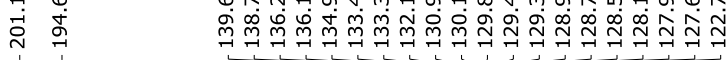

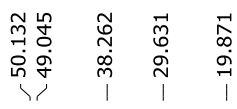
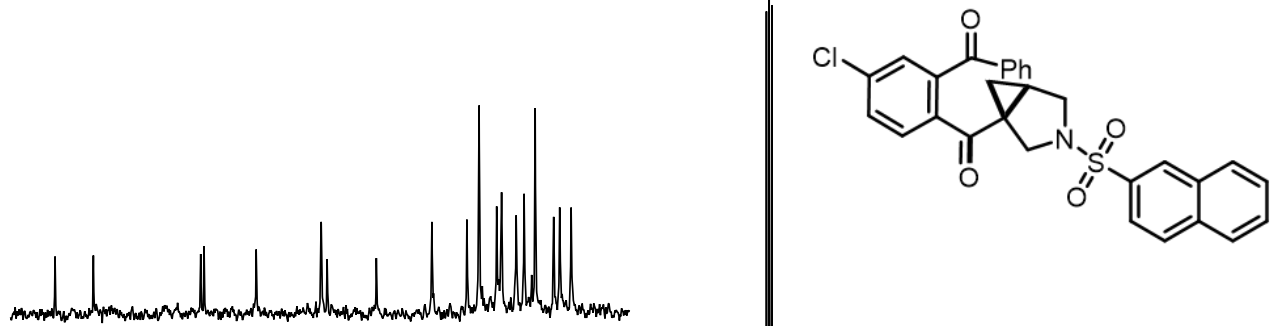

140139138137136135134133132131130129128127

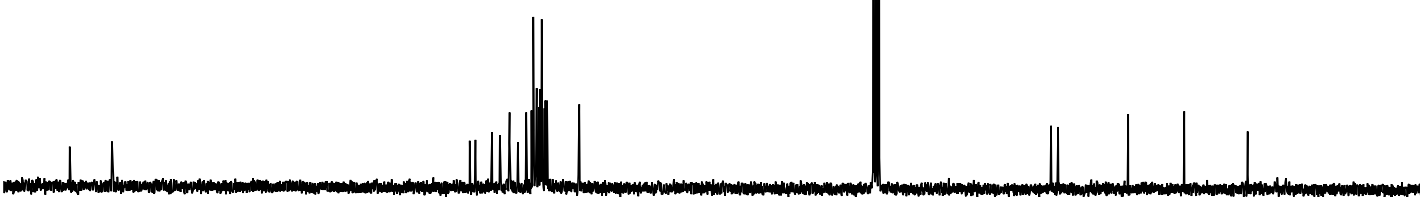

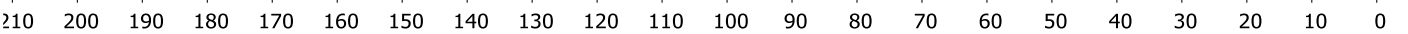

${ }^{13} \mathrm{C}$ NMR Compound $4 \mathbf{e}\left(75 \mathrm{MHz}, \mathrm{CDCl}_{3}\right)$ 


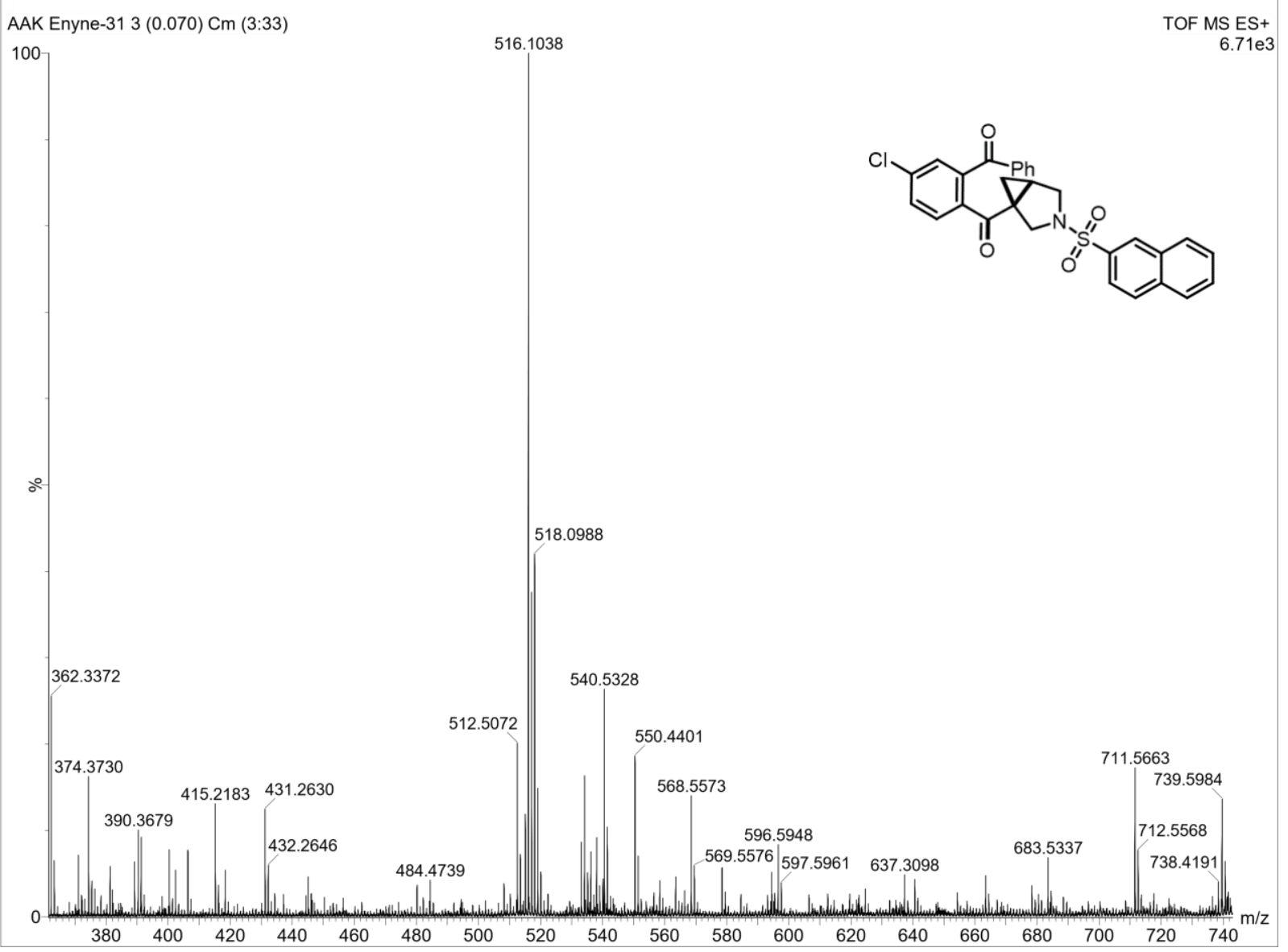

Mass-ESI Compound $\mathbf{4 e}$ 